\title{
Ergebnisse der Erhebung: Die zivilgesellschaftlichen Performanzen der MSO
}

\subsection{Deskriptive Bestandsaufnahme, integrierte Hypothesenprüfung und Diskussion der Ergebnisse}

\subsubsection{Allgemeine Charakteristika der MSO}

\section{Heimatkommunen der MSO}

Der Tab. 7.1 ist zu entnehmen, dass die meisten Rückläufer aus den Städten Dortmund $(18 \%)$ und Bielefeld $(16 \%)$ kamen. ${ }^{1}$ Neben den neun für die Erhebung vorgesehenen Kommunen Dortmund, Essen, Duisburg, Bochum, Gelsenkirchen, Bielefeld, Oberhausen, Hagen und Hamm wurde in fünf weiteren Städten Köln, Düsseldorf, Bergkamen, Bocholt, Herne und Solingen an der Befragung teilgenommen. Diese Tatsache ist damit zu erklären, dass sich die Sitze der angeschriebenen Organisationen an anderen Orten befanden und/oder die Anfrage weitergeleitet wurde. Zudem waren auf den von der Studienleiterin für die Bewerbung der Teilnahme an der Befragung in den Erhebungskommunen besuchten Netzwerktreffen der MSO immer auch Organisationen aus anderen Städten anwesend, deren Teilnahme nicht kategorisch ausgeschlossen werden sollte.

\footnotetext{
${ }^{1}$ Zur Erleichterung der Lesbarkeit werden die Prozentwerte im Fließtext konsequent auf ganze Zahlen gerundet.
}

Elektronisches Zusatzmaterial Die elektronische Version dieses Kapitels enthält Zusatzmaterial, das berechtigten Benutzern zur Verfügung steht https://doi.org/10.1007/978-3-658-34298-2_7. 
Tab.7.1 Fallzahlen (Rückläufer) in den Kommunen

\begin{tabular}{lcc}
\hline & Häufigkeit & Prozent \\
\hline Dortmund & 47 & 18,3 \\
\hline Bielefeld & 41 & 16,0 \\
\hline Bochum & 38 & 14,8 \\
\hline Essen & 38 & 14,8 \\
\hline Duisburg & 20 & 7,8 \\
\hline Köln & 16 & 6,2 \\
\hline Oberhausen & 15 & 5,8 \\
\hline Hamm & 15 & 5,8 \\
\hline Gelsenkirchen & 13 & 5,1 \\
\hline Hagen & 8 & 3,1 \\
\hline Düsseldorf & 2 & 0,8 \\
\hline Bergkamen & 1 & 0,4 \\
\hline Bocholt & 1 & 0,4 \\
\hline Herne & 1 & 0,4 \\
\hline Solingen & 1 & 0,4 \\
\hline Gesamt & 257 & 100,0 \\
\hline
\end{tabular}

Gründungsjahre: Religiöse MSO sind älter

Die Gründungsjahre der MSO sind sehr breit gestreut (1947 bis 2018) und wurden in Dekaden zusammengefasst (Abb. 7.1). Es zeigt sich, dass sich die befragten MSO mit 74 Prozent in erster Linie in den letzten 29 Jahren gegründet haben (1991-2000; 2001-2010; 2011-2018). Allerdings ist es auch sehr beachtlich, dass einige der Organisationen bereits seit 40 bis 50 Jahren und länger existieren.

Der $t$-Test zeigt, dass sich die religiösen und nicht-religiösen MSO bezüglich ihres Alters signifikant unterscheiden, wobei die religiösen im Durchschnitt älter sind. ${ }^{2}$

\footnotetext{
${ }^{2}$ Nicht-religiöse Gruppe: $M=2000,28 ; S D=13,646 ; \mathrm{n}_{\mathrm{S}}=174$; religiöse Gruppe: $M=1994,85 ; S D=15,043 ; \mathrm{n}_{\mathrm{R}}=78 \mid t(250)=2,831 ; p=0,005 ;$ Cohen's $d=0,39$.
} 


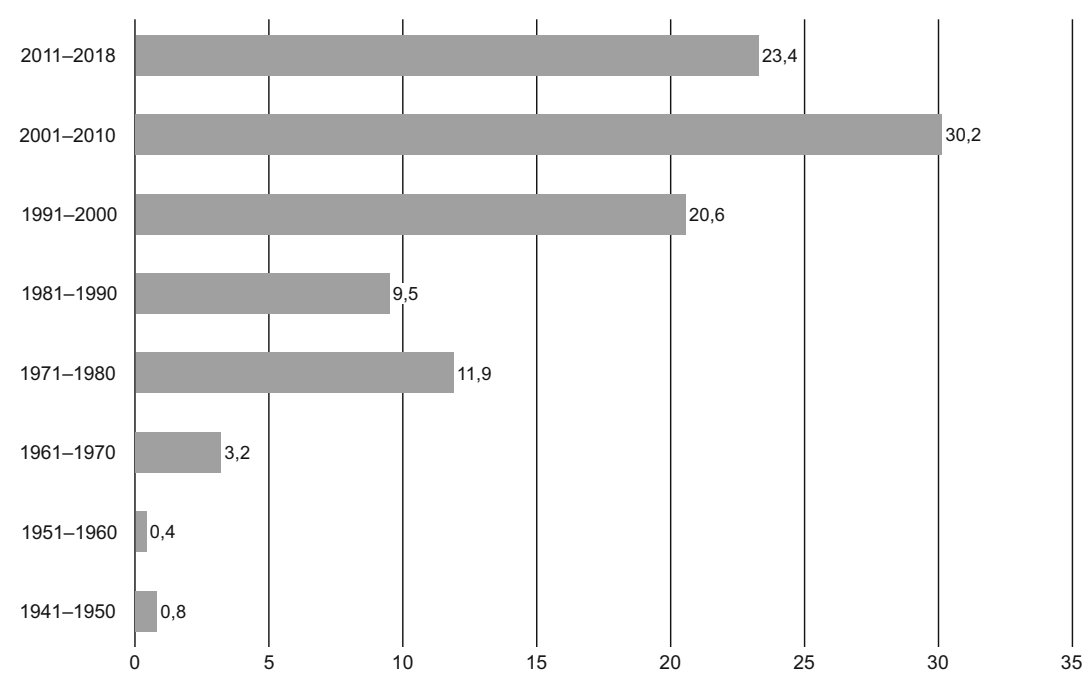

$n=252$

Abb. 7.1 Gründungsjahre der MSO in Dekaden (in \%)

Rechtsformen: Vereine als dominante Organisationsform

Mit rund 53 Prozent ist die Mehrheit der befragten MSO der Rechtsform des eingetragenen Vereins (e. V.) zuzuordnen, bei 42 Prozent der MSO handelt es sich um gemeinnützige Vereine (Tab. 7.2). Diese profitieren von Steuervergünstigungen, weil die Satzungen und Tätigkeiten der Vereine den hierfür notwendigen, in der Abgabenordnung (AO) $\S \S 51 \mathrm{ff}$. formulierten Voraussetzungen entsprechen (BMJV 2016: 60; II. 4.3.1). 
Tab. 7.2 Rechtsformen der MSO

\begin{tabular}{lcc}
\hline & Häufigkeit & Prozent \\
\hline Eingetragener Verein (e.V.) & 136 & 52,9 \\
\hline Gemeinnütziger Verein & 108 & 42,0 \\
\hline Körperschaft des öffentlichen Rechts & 4 & 1,6 \\
\hline Einzelorganisation & 3 & 1,2 \\
\hline Dachverband & 4 & 1,6 \\
\hline Sonstige & 2 & 0,8 \\
\hline Gesamt & 257 & 100,0 \\
\hline
\end{tabular}

Bei den Dachverbänden und Körperschaften des öffentlichen Rechts handelt es sich ausschließlich um religiöse MSO; die säkularen Vereinigungen konstituieren sich häufiger als gemeinnützige Vereine (MO: $46 \%$ vs. RMO: $33 \%{ }^{3}$ ).

\section{Mitgliedschaften in 53 verschiedenen Dachverbänden}

129 der befragten MSO (51 \%) gehören einem Dachverband an. Von diesen benannten in der Folgefrage 125 den Dachverband oder auch die Mitgliedschaft in mehreren Dachorganisationen (Mehrfachnennung). Genannt wurden insgesamt 53 unterschiedliche Dachverbände; bei 19 von ihnen handelt es sich um religiöse Dachorganisationen ${ }^{4}$. Mit 34 Nennungen (27\%) seitens säkularer MSO am häufigsten angeführt wurde eine Mitgliedschaft im Paritätischen Wohlfahrtsverband ${ }^{5}$. Auf Platz zwei folgt der Verbund sozial-kultureller Migrantenvereine Dortmund e. V. (VMDO) mit 14 Nennungen (11\%) und die Türkisch-Islamische Union der Anstalt für Religion e. V. (DİTiB) mit insgesamt 10 Anführungen (8\%) auf Platz drei. Weitere häufiger genannte Dachverbände sind in 7 Fällen die Islamische Gemeinschaft Millî Görüş̧ e. V. (IGMG) (6\%) sowie jeweils mit 5 Nennungen (4\%) die Islamische Gemeinschaft der Bosniaken in Deutschland (IGBD) sowie der Essener Verbund der Immigrantenvereine e. V.

\footnotetext{
${ }^{3}$ Zur Ausweisung von zu einer jeweiligen Organisationskategorie gehörigen Prozentwerten werden im Folgenden auch die Kürzel RMO (für religiöse) und MO (für nicht-religiöse) verwendet (6.2).

${ }^{4}$ Siehe für eine detaillierte Auflistung der 53 säkularen und religiösen Dachverbände Tab. A 1 im elektronischen Zusatzmaterial.

${ }^{5}$ Auch der Studie des SVR-Forschungsbereichs zufolge sind recht viele der MSO (23\%) Mitglied in einem Wohlfahrtsverband, und zwar insbesondere im Paritätischen Wohlfahrtsverband (Friedrichs et al. 2020: 54 f.; vgl. auch Halm et al. 2020: 122).
} 
Zuordnung zu einer religiösen Tradition oder Lehre: Prävalenz islamischer MSO

80 und damit 31 Prozent der 257 befragten Funktionsträger ordnen ihre Organisationen einer religiösen Tradition oder Lehre zu, die im Rahmen der vorliegenden Arbeit folglich als religiöse MSO kategorisiert werden. 75 der sich einer religiösen Tradition zuordnenden MSO beantworteten die Frage, ob es ein Hauptanliegen der Organisation sei, die Tradition bzw. Lehre gemeinsam zu pflegen und nach außen zu verkünden. ${ }^{6}$ Dies trifft für 60 der religiösen Vereinigungen $(80 \%)$ $\mathrm{zu}$.

In einer darauffolgenden Frage wurden die religiösen MSO gebeten, die religiösen Traditionen zu spezifizieren. Die diesbezüglich getätigten Angaben lassen sich den in der Tab. 7.3 aufgeführten übergeordneten Konfessionen zuordnen. Mit 55 Prozent mit Abstand am häufigsten vertreten ist der Islam, gefolgt vom Christentum (21\%) und dem Alevitentum (15\%) .

Tab. 7.3 Zuordnung der MSO zu religiösen Traditionen

\begin{tabular}{lcc}
\hline & Häufigkeit & Prozent \\
\hline Islam & 44 & 55,0 \\
\hline Christentum & 17 & 21,3 \\
\hline Alevitentum & 12 & 15,0 \\
\hline Ezidentum & 3 & 3,8 \\
\hline Judentum & 3 & 3,8 \\
\hline Hinduismus & 1 & 1,3 \\
\hline Religiöse MSO & 80 & 100,0 \\
\hline Nicht-religiöse MSO & 177 & \\
\hline Gesamt & 257 & \\
\hline
\end{tabular}

\footnotetext{
${ }^{6}$ Damit lässt sich überprüfen, ob sich eine religiöse MSO als eine „Religionsgemeinschaft“ versteht (Anschütz 1933, Art. 137, S. 633; 6.1). Dies impliziert ein umfassendes religiöses Selbstverständnis mit umfangreichen Zielsetzungen, die über die Pflege lediglich partieller religiöser Angelegenheiten hinausgehen, wie sie von religiösen Vereinen oder anderen Zusammenschlüssen zu erwarten wäre.

${ }^{7}$ Entsprechend der Selbstauskünfte der MSO werden „Islam“ und „Alevitentum“ bzw. „Muslime“ und „Aleviten“ im Rahmen dieser Arbeit konsequent separat aufgeführt. Damit ist ausdrücklich keine Positionierung zu einer Nichtzugehörigkeit der Aleviten zum Islam intendiert. Ob sich Aleviten dem Islam zugehörig fühlen und sich als Muslime bezeichnen (sollten), ist ein kontrovers diskutiertes und im Rahmen dieser Arbeit nicht weiter auszuführendes Thema.
} 
Die Tradition „Islam“ wurde zumeist explizit genannt, ihr wurde im Rahmen der Auswertung aber auch der lediglich 2-mal angeführte Sufismus zugeordnet. Mit der Kategorie „Christentum“ wurden die Angaben „Christentum“, „Evangelium“, „evangelisch-freikirchlich“, „Freie evangelische Pfingstgemeinde“ und „Mennoniten-Brüdergemeinde“ zusammengefasst. Des Weiteren wurden ihr aber auch die „Orthodoxen Kirchen“ mit den 5 Nennungen „christlich-orthodox“, „griechisch-orthodox“, „Armenische christliche Tradition“, „Russisch-orthodoxe Kirche“ und „Syrisch-orthodoxe Kirche von Antiochien“" zugeordnet. Darüber hinaus wurden zu deutlich geringeren Anteilen jeweils dreimal das „Ezidentum“ und das ,Judentum“ und einmal der „Hinduismus“ genannt.

Von den insgesamt 44 islamischen MSO weisen 28 (64\% der islamischen) eine Verbandszugehörigkeit auf. Wie bereits erwähnt gehören 10 Vereinigungen der Türkisch-Islamischen Union der Anstalt für Religion e. V. (DİTIBB) an, 7 der Islamischen Gemeinschaft Millî Görüş (IGMG), 5 der Islamischen Gemeinschaft der Bosniaken in Deutschland e. V. (IGBD), 2 dem Verband der Islamischen Kulturzentren e. V. (VIKZ), 2 der Islamischen Religionsgemeinschaft NRW e. V. (IRG), 2 dem Verband engagierte Zivilgesellschaft in NRW e. V. (VEZ NRW) (darunter ist eine MSO, die auch in der IGMG Mitglied ist) sowie eine der African Ummah Europe.

\subsubsection{Ressourcenausstattungen}

\subsubsection{Die Mitglieder in Zahlen: nach Personengruppen, Herkunftsländern, Konfessionen}

Religiöse MSO haben signifikant mehr Mitglieder

Mit der Mitgliedschaftsentscheidung einher geht eine subjektive freiwillige „Selbstverpflichtung“ (Luhmann 1964: 37), der Mitgliedsrolle und damit verknüpften Verhaltenserwartungen zu entsprechen. Dieses Commitment ist konstitutiv für den Bestand einer Organisation (II. 4.3.3). Es ist anzunehmen, dass die Funktionsträger bei der Angabe der Mitgliederzahlen zwischen den in ihren Organisationen vorzufindenden Mitglieder- und Beteiligungsstrukturen nicht immer streng unterschieden haben. Thematisiert wurde in diesem Zusammenhang das sich vom „deutschen Idealverein“ unterscheidende Verhältnis zwischen Mitglieder- und Beteiligungsstruktur in migrantischen und Moscheevereinen: In diesen wird eine kleine Anzahl eingetragener beitragszahlender und über die satzungsgemäßen Pflichten und Rechte verfügenden Mitglieder von einer weitaus 
größeren Anzahl von (un-)regelmäßig Engagierten, Moscheebesuchern und anderweitig Beteiligten flankiert. Formal sind diese Nicht-Mitglieder, nehmen am Vereinsleben aber aktiv teil (II. 4.3.3).

Jeweils ein Drittel der befragten MSO haben 11 bis 50 (33\%) oder mehr als 100 Mitglieder (33\%) (Tab. 7.4).

Tab.7.4 Mitgliederzahlen

\begin{tabular}{lcc}
\hline Mitglieder & Häufigkeit & Prozent \\
\hline unter 10 & 22 & 8,6 \\
\hline 11 bis 50 & 85 & 33,2 \\
\hline 51 bis 100 & 65 & 25,4 \\
\hline mehr als 100 & 84 & 32,8 \\
\hline Gesamt & 256 & 100,0 \\
\hline
\end{tabular}

Die unter dem Item „mehr als 100 Mitglieder, und zwar “ in 74 Fällen konkretisierten Angaben wurden in weitere einzelne Gruppen eingeteilt, die der Tab. $7.5 \mathrm{zu}$ entnehmen sind. Es zeigt sich, dass 50 Prozent der MSO 101 bis 200 Mitglieder haben, jeweils rund 15 Prozent über 201 bis 300, 301 bis 600 und rund 14 Prozent über 601 bis 1.000 Mitglieder verfügen. In rund 7 Prozent der MSO fallen die Mitgliederzahlen mit über 1.000 Personen vergleichsweise hoch aus. Sehr hohe Zahlen von 2.000 und mehr Mitgliedern sind ausschließlich religiösen MSO zuzuordnen. So gibt die der Syrisch-orthodoxen Kirche von Antiochien zugehörigen MSO 2.000 Mitglieder an, eine islamische Organisation 5.000, eine hinduistische 6.000 und eine alevitische MSO, die dem Bund der Alevitischen Jugendlichen in Deutschland e. V. (BDAJ) angehört, vermerkt eine Zahl von 16.500 Mitgliedern. Auch in der Gruppe 601 bis 1.000 Mitglieder sind mit 8 Fällen mehrheitlich religiöse Organisationen vertreten: 3 islamische, 3 alevitische, eine jüdische und eine Mennoniten-Brüdergemeinde.

Ergebnisse des $U$-Tests zeigen, dass die religiösen Vereinigungen mit einem mittleren Effekt signifikant mehr Mitglieder haben. ${ }^{8}$

\footnotetext{
${ }^{8}$ Berechnung des $U$-Tests mit vierstufiger ordinalskalierter Variable (, unter 10‘; 11 bis $50^{\text {‘; }}$ ,51 bis $100^{`} ;$, mehr als 100'); $U=3477 ; z=-6,8 ; p=0,000 ; r=0,42 ; \mathrm{Md}_{\mathrm{S}}=8 ; \mathrm{Md}_{\mathrm{R}}=10$.
} 
Tab. 7.5 Mitgliederzahlen über 100 (in absoluten und relativen Häufigkeiten)

\begin{tabular}{lcccc}
\hline & $\begin{array}{c}\text { Alle MSO } \\
\text { (absolute Zahlen) }\end{array}$ & in \% & $\begin{array}{c}\text { Nicht-religiöse MSO } \\
\text { (absolute Zahlen) }\end{array}$ & $\begin{array}{c}\text { Religiöse MSO } \\
\text { Zabl-150 }\end{array}$ \\
\hline $101-20$ & 27,0 & 11 & 9 \\
\hline $151-200$ & 17 & 23,0 & 7 & 6 \\
\hline $201-300$ & 11 & 14,9 & 5 & 6 \\
\hline $301-600$ & 11 & 14,9 & 5 & 4 \\
\hline $601-1.000$ & 10 & 13,5 & 2 & 43 \\
\hline Über $1.000^{*}$ & 5 & 6,8 & 1 & 41 \\
\hline
\end{tabular}

* Organisationen mit über 1.000 Mitgliedern: 1.052, 2.000, 5.000, 6.000, 16.500 Mitglieder.

Personengruppen: Vielfältig durchmischte Mitgliederstruktur mit Dominanz von Berufstätigen

Die Funktionsträger der MSO wurden gebeten, alle Mitglieder ihrer Organisation mit einer sechsstufigen Antwortskala ,alle“ (Code 1), „mehr als die Hälfte“ (2), „,ungefähr die Hälfte“ (3), „weniger als die Hälfte“ (4), „,keine“ (5) und „,weiß nicht“ (6) den 5 Personenkreisen „Berufstätige/Selbstständige“, „Schüler und Studierende“, „Hausfrauen/Hausmänner“, „Arbeitslose/Arbeitssuchende“ oder „Rentner“ zuzuordnen. Die Einteilungen der Mitglieder in die vorgegebenen Personenkreise ${ }^{9}$ beruhen auf Schätzungen und sie funktionierten erstaunlich gut. Sie spiegeln ein breites Spektrum an Mischungsverhältnissen, deren ausführliche Darstellung aufgrund zahlreicher und einzelner Gruppenkonstellationen an dieser Stelle nicht sinnvoll ist. Ein wesentliches Erkenntnisinteresse ist gewesen, die unter den Mitgliedern einzeln oder dominant vertretenen Personenkreise zu identifizieren. Zu diesem Zweck wurden die mit der Skala im Fragebogen pro Personengruppe vorgenommenen Einstufungen unter Angabe von Mehr- oder Minderheits- sowie ausgeglichenen (,paritätischen“) Verhältnissen in eine nominalskalierte Variable überführt. ${ }^{10}$ Durch die Zusammenführung der Antworten war in einem ersten Schritt ganz allgemein die Identifikation von in den einzelnen MSO durch die Mitglieder überhaupt präsenten Statuskreisen möglich. Die grobe Auszählung nach Gruppen zeigt, dass die Mitglieder in den meisten

\footnotetext{
${ }^{9}$ Die Begriffe „Personenkreise“ und „Personengruppen“ werden im Rahmen dieser Arbeit synonym verwendet.

${ }^{10}$ Die in der Variable aufgeführten Informationen lauten z. B.: „Alle Berufstätige“; oder: „Berufstätige (2), Schüler und Studierende \& Hausfrauen/-männer \& Arbeitslose \& Rentner (4)“ [Codierungen $2=$ mehr als die Hälfte; 4 = weniger als die Hälfte].
} 
Organisationen 5 Statusgruppen zuzuordnen sind und dass zwischen den einzelnen Gruppenpräsenzen sprunghafte prozentuale Anstiege zu registrieren sind ${ }^{11}$ (Abb. 7.2).

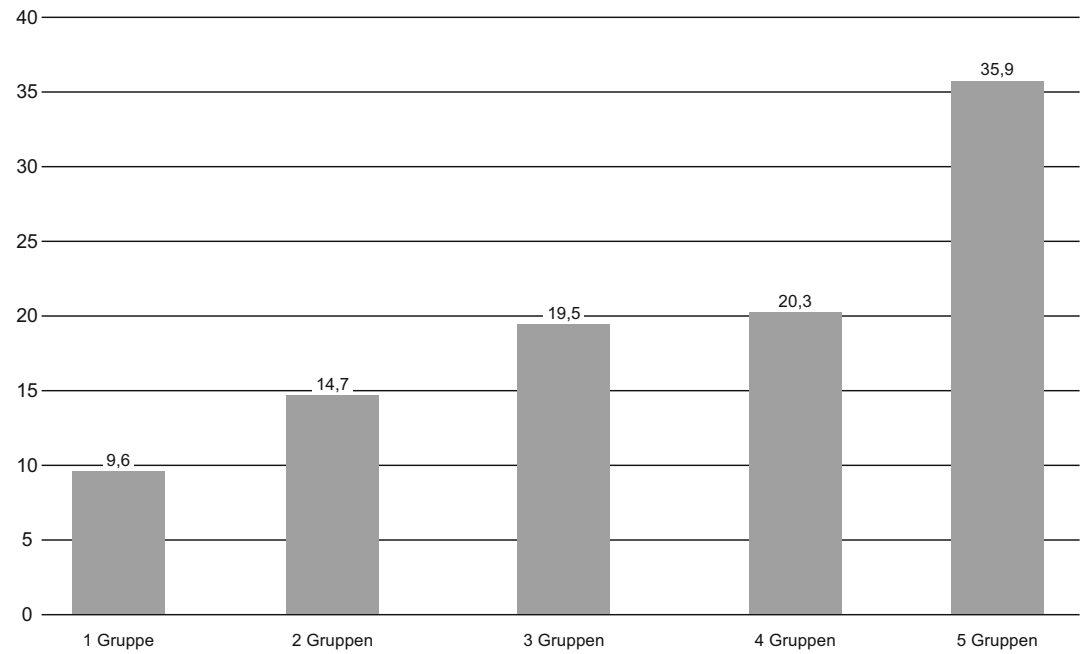

$n=251$

Abb. 7.2 Verteilung der Mitglieder auf Anzahl der Personengruppen (in \%)

Eine kleinteiligere Betrachtung fördert zutage, aus welchen Personenkreisen und wie vielfältig sich die Mitglieder jeweils zusammensetzen (Tab. 7.6). Die Dominanz von 5 Personenkreisen $(36 \%$ ) wird mit einem großen prozentualen Abstand gefolgt von der Präsenz von 4 Gruppen (ohne Arbeitslose bzw. ohne Rentner etc.) sowie der ausschließlich Berufstätige umfassenden Gruppe (8\%). Weitere vorherrschende Mitgliederstrukturen betreffen mit einem Anteil von 5 Prozent (und darunter) Zweier- und Dreier-Gruppen mit jeweils unterschiedlichen Personenzusammensetzungen (Tab. 7.6).

\footnotetext{
${ }^{11}$ Bei der Auswertung wurden nur getätigte Angaben einbezogen; fehlende Antworten wurden als Residualkategorie wie eine Nichtzustimmung behandelt.
} 
Tab.7.6 Mitglieder nach Personengruppen (bzw. Personenkreisen)

\begin{tabular}{lcc}
\hline & Häufigkeit & Prozent \\
\hline 5 Gruppen & 90 & 35,9 \\
\hline 4 Gruppen (o. Arbeitslose) & 24 & 9,6 \\
\hline 4 Gruppen (o. Rentner) & 16 & 6,4 \\
\hline 4 Gruppen (o. SuS ${ }^{*}$ ) & 9 & 3,6 \\
\hline 4 Gruppen (o. Berufstätige) & 1 & 0,4 \\
\hline 4 Gruppen (o. Hausfrauen) & 1 & 0,4 \\
\hline 3 Gruppen: Berufstätige, Hausfrauen, Rentner & 12 & 4,8 \\
\hline 3 Gruppen: Berufstätige, SuS, Rentner & 12 & 4,8 \\
\hline 3 Gruppen: Berufstätige, SuS, Hausfrauen & 11 & 4,4 \\
\hline 3 Gruppen: Berufstätige, SuS, Arbeitslose & 7 & 2,8 \\
\hline 3 Gruppen: Berufstätige, Arbeitslose, Rentner & 3 & 1,2 \\
\hline 3 Gruppen: Berufstätige, Hausfrauen, Arbeitslose & 3 & 1,2 \\
\hline 3 Gruppen: SuS, Hausfrauen, Arbeitslose & 1 & 0,4 \\
\hline 2 Gruppen: Berufstätige, Rentner & 13 & 5,2 \\
\hline 2 Gruppen: Berufstätige, SuS & 11 & 4,4 \\
\hline 2 Gruppen: Berufstätige, Arbeitslose & 4 & 1,6 \\
\hline 2 Gruppen: Berufstätige, Hausfrauen & 4 & 1,6 \\
\hline 2 Gruppen: SuS, Hausfrauen & 2 & 0,8 \\
\hline 2 Gruppen: Hausfrauen, Arbeitslose & 1 & 0,4 \\
\hline 2 Gruppen: SuS, Arbeitslose & 1 & 0,4 \\
\hline 2 Gruppen: SuS, Rentner & 1 & 0,4 \\
\hline 1 Gruppe: Berufstätige & 19 & 7,6 \\
\hline 1 Gruppe: SuS & 2 & 0,8 \\
\hline 1 Gruppe: Arbeitslose & 1 & 0,4 \\
\hline 1 Gruppe: Hausfrauen & 1 & 0,4 \\
\hline 1 Gruppe: Rentner & 251 & 0,4 \\
\hline Gesamt & 100,0 \\
\hline
\end{tabular}

${ }^{*}$ SuS $=$ Schüler und Studierende.

Neben der Anzahl vorhandener Gruppen wurden im Rahmen eines zweiten Analyseschritts zusätzlich auch Mehrheitsverhältnisse identifiziert, z. B. „5 Gruppen: mehrheitlich Berufstätige“. Die kleinteiligere Betrachtung von unter den Mitgliedern vertretenen Gruppen nach einem jeweils dominierenden Personenkreis oder paritätisch vertretenen Anteilen zeigt, dass es sich bei den Mitgliedern - über alle Gruppenkombinationen hinweg - mehrheitlich um berufstätige bzw. 
selbstständige Personen handelt. In 14 Prozent der MSO sind alle 5 Gruppen zu gleichen (paritätischen) Anteilen vertreten (ohne Abb.). ${ }^{12}$

Die Melange der verschiedenen Statusgruppen unter den Mitgliedern verdeutlicht, dass in den einzelnen MSO Menschen unterschiedlichen Alters und Erwerbsstatus involviert sind. Auch Arbeitslose sind eingeschlossen. Homogene Verhältnisse bestehen nur in Bezug auf Berufstätige bzw. Selbstständige. Interessant wäre an dieser Stelle eine Vergleichsziehung zu den in den Selbstorganisationen von Autochthonen vorhandenen Mitgliedschaftsstrukturen. Diese ist aufgrund fehlender Daten jedoch nicht möglich.

\section{Herkunftsländer: Ein Herkunftsland in 49 Prozent der MSO}

Bei der Frage, aus welchen Herkunftsländern die Mitglieder überwiegend kommen, waren mehrfache und auch freie Antworten (,Sonstiges“) zugelassen. Die Option des eigenständigen Hinzufügens wurde häufig genutzt. Unter einer Vielzahl von Herkunftsländern (Mehrfachnennung) am häufigsten angegeben wurde die Türkei (44\%), gefolgt von afrikanischen Staaten (28\%), unter denen Marokko (13\%) hervortritt. Deutschland (19\%), Syrien (18\%), Russland (15\%) und Irak (12\%) wurden ebenfalls vermehrt markiert bzw. unter ,Sonstige“ aufgeführt. Weitere des Öfteren vermerkte Länder sind Griechenland (9\%), Polen und Afghanistan (7\%), die Ukraine und Kasachstan (jeweils $4 \%$ ), Italien (3\%) und mit jeweils 3 Prozent Indonesien, Bosnien bzw. Bosnien und Herzegowina, Serbien und Montenegro (Ex-Jugoslawien) sowie die ehemaligen UDSSR-Republiken Usbekistan, Tadschikistan und Kirgisistan. In jeweils 2 Prozent der MSO stammen die Mitglieder auch aus dem Kosovo, dem Libanon, Albanien, Palästina, Mazedonien, Bulgarien und Indien.

Die insgesamt gemachten Angaben wurden nach der Anzahl von Ländern kategorisiert. In 49 Prozent der MSO - und damit mit Abstand am häufigsten - kommen die Mitglieder aus einem einzigen Herkunftsland (Tab. 7.7), wobei es sich bei 59 dieser MSO (48\%; $\mathrm{n}=122$ ) um die Türkei handelt. In 11 Prozent der herkunftshomogenen Organisationen stammen die Mitglieder aus einem afrikanischen Land, in 8 Prozent ausschließlich aus Russland, in 7 Prozent alleinig aus Griechenland und in 5 Prozent aus Deutschland. ${ }^{13}$ Für 15 Prozent werden

${ }^{12}$ Inkonsistente Angaben, wie etwa das Markieren der Antwortmöglichkeit ,alle“ oder ,ungefähr die Hälfte“ für alle fünf Personengruppen, wurden vermittels einer Uminterpretation der Angabe berücksichtigt, indem davon ausgegangen wird, dass alle Gruppen zu gleichwertigen Anteilen in der betreffenden Organisation vertreten sind. Dies wurde dann wie folgt vermerkt: ,5 Gruppen: paritätische Anteile“.

${ }^{13}$ Die in sechs Fällen gemachte Angabe, dass die Mitglieder ausschließlich aus Deutschland kommen, lässt zunächst darauf schließen, dass es sich nicht um eine MSO im definierten 
zwei Länder, für 10 Prozent drei und für 6 Prozent vier Länder angegeben. In 5 Prozent der MSO stammen die Mitglieder aus acht und mehr Herkunftsländern und von weiteren 5 Prozent wurde ausschließlich ein Kontinent genannt oder auf die internationale Zusammensetzung der Mitglieder verwiesen (Tab. 7.7).

Tab. 7.7 Anzahl der Herkunftsländer der Mitglieder

\begin{tabular}{lcc}
\hline & Häufigkeit & Prozent \\
\hline ein Land & 122 & 48,6 \\
\hline 2 Länder & 38 & 15,1 \\
\hline 3 Länder & 25 & 10,0 \\
\hline 4 Länder & 16 & 6,4 \\
\hline 5 Länder & 10 & 4,0 \\
\hline 6 Länder & 7 & 2,8 \\
\hline 7 Länder & 7 & 2,8 \\
\hline 8 Länder und mehr & 13 & 5,2 \\
\hline Nennung Kontinent oder „international“ & 13 & 5,2 \\
\hline Gesamt & 251 & 100,0 \\
\hline
\end{tabular}

Für circa die Hälfte der MSO ist hinsichtlich der Mitglieder somit eine Herkunftslandhomogenität festzustellen. Diese lässt sich für 62 Prozent und damit mehrheitlich für die sich einer religiösen Tradition zuordnenden MSO ausmachen (vs. MO: $42 \%$ ). Darüber hinaus zeigt sich bei 39 der religiösen MSO (50 \%) eine doppelte Homogenität durch die Koinzidenz von herkunftslandbezogener und konfessioneller Konformität der Mitglieder. Auf die Konfessionszugehörigkeiten der Mitglieder wird im folgenden Abschnitt näher eingegangen.

Sinn handelt (I. 1; II. 4.1). Die betreffenden MSO wurden jedoch im Datensatz belassen, da im Anschreiben explizit formuliert war, dass sich die Umfrage an Organisationen richtet, die „von einst zugewanderten Menschen und ihren Nachkommen“ gegründet worden sind. Zudem kann es sich um in Deutschland geborene Mitglieder mit einem Migrationshintergrund der zweiten oder dritten Generation handeln. Des Weiteren ließ die Prüfung der Beantwortung weiterer Fragen die Annahme zu, dass es sich in den betreffenden Fällen um migrantische Vereinigungen handelt.

Weitere einzelne Herkunftsländer betreffen Italien (3\%), Syrien, Bosnien und Herzegowina, (Süd-)Korea, Palästina (jeweils $2 \%$ ), Kosovo, Kroatien, Serbien und Montenegro (Ex-Jugoslawien), Irak, Afghanistan, Libanon, Indien, Sri Lanka, Bangladesch, Japan, Frankreich, Spanien (jeweils $1 \%$ ). 
Jenseits einer für die Hälfte der MSO festzustellenden Herkunftslandhomogenität verweisen die Ergebnisse auf eine in den anderen MSO vorzufindende ethnische Durchmischung und Pluralität. Neben den Türkeistämmigen sind afrikanische und andere Communities bedeutsam.

Angesichts der Ergebnisse lässt sich vermuten, dass MSO in besonderer Weise fähig sind, Rahmenbedingungen zu schaffen, um Menschen unterschiedlicher Nationalitäten zu inkorporieren. Dies sollte abgrenzenden Verhaltensweisen entgegenstehen. Jüngere Untersuchungen haben für Regionen mit einer hohen Zuwanderungsdichte eine stärkere kulturelle Durchmischung und Vielfalt der MSO festgestellt (Priemer et al. 2017: 23). Dies kann auch für die vorliegende Studie und damit für den nordrhein-westfälischen Kontext geltend gemacht werden. Zudem ist sich vor Augen zu führen, dass derartig , bunte' Zusammensetzungen in Organisationen und Dienstleistungseinrichtungen von Menschen ohne Zuwanderungsgeschichte in Deutschland in deutlich geringerem Ausmaß auszumachen sein sollten, was im Zusammenhang mit einer mangelhaften und wenig vorangeschrittenen interkulturellen Öffnung thematisiert wird (ebd.: 41; Hunger/Metzger 2013a; Jähnichen et al. 2016). Insofern können die MSO in der Einwanderungsgesellschaft Deutschland mit Blick auf interkulturelle Öffnungsund Lernprozesse als Beispiele guter Praxis fungieren.

Konfessionszugehörigkeiten: Konfessionelle Homogenität in 38 Prozent der MSO

Eine weitere, für einige Funktionsträger sicherlich herausforderungsvolle Aufgabe, bestand darin, die Zugehörigkeit der Mitglieder zu einer bestimmten Glaubensrichtung einzuschätzen. Neben 15 verschiedenen Glaubensrichtungen konnten zwei weitere Items (,konfessionslos“ und „Sonstige“) mit einer sechsstufigen Antwortskala ${ }^{14}$ eingestuft werden. Von besonderem Erkenntnisinteresse ist hier in einem ersten Schritt zunächst die Identifizierung von dominanten sowie homogenen und heterogenen Verhältnissen in Bezug auf die Religionszugehörigkeiten der Mitglieder in allen MSO sowie in einem zweiten Schritt auch vor dem Hintergrund der Zuordnung von 80 MSO zu einer bestimmten religiösen Tradition. Bei der Beantwortung der Frage fielen die Kombinationsmöglichkeiten noch höher aus als bei der Einteilung der Mitglieder in die fünf Personenkreise. Auch bei der Auswertung der Konfessionszugehörigkeiten wurden die getätigten Angaben in eine nominalskalierte Variable überführt und in einem weiteren Schritt nach einzelnen, dominant oder zu paritätischen Anteilen vertretenen religiösen Überzeugungen kategorisiert. Die so erfolgte Einteilung zeigt (Tab. 7.8),

\footnotetext{
14 Antwortskala: ,alle“, ,,mehr als die Hälfte“, ,,ungefähr die Hälfte“, ,,weniger als die Hälfte“, „keine“ und „weiß nicht“.
} 
dass in 21 Prozent der MSO alle Mitglieder sunnitisch und in 15 Prozent die Mitglieder mehrheitlich sunnitisch sind. In 11 Prozent der Organisationen gehören alle Mitglieder einer einzigen Glaubensrichtung ${ }^{15}$ an und in 13 Prozent der MSO mehrheitlich einer Konfession ${ }^{16}$. Zu einem dominanten Anteil konfessionslos sind die Mitglieder in 8 Prozent der MSO. Weitere nach Gemeinsamkeiten vorgenommene Unterteilungen sind der Tab. $7.8 \mathrm{zu}$ entnehmen. In Bezug auf die nicht ausgewiesenen fehlenden Angaben ist an dieser Stelle anzumerken, dass hierunter im Rahmen der Auswertung der Religionszugehörigkeiten generell sowohl

Tab. 7.8 Kategorisierung der Mitglieder nach Konfessionszugehörigkeiten

\begin{tabular}{lcc}
\hline Die Mitglieder sind ... & Häufigkeit & Prozent \\
\hline Alle sunnitisch & 48 & 21,1 \\
Mehrheitlich sunnitisch & 33 & 14,5 \\
\hline Alle Anhänger einer Konfession* & 24 & 10,5 \\
Mehrheitlich Anhänger einer Konfession** & 29 & 12,7 \\
\hline Mehrheitlich konfessionslos & 18 & 7,9 \\
\hline Alle alevitisch & 9 & 3,9 \\
Mehrheitlich alevitisch & 9 & 3,9 \\
\hline Alle römisch-katholisch & 6 & 2,6 \\
Mehrheitlich römisch-katholisch & 12 & 5,3 \\
\hline Anhänger von 2 Konfessionen (zu gleichen Anteilen) & 16 & 7,0 \\
\hline Anhänger von 3, 4 oder 6 Konfessionen & 11 & 4,8 \\
(zu gleichen Anteilen) & 10 & 4,4 \\
\hline Konfessionslos und Anhänger von bis zu 6 Konfessionen & 3 & 1,3 \\
\hline (zu gleichen Anteilen) & 228 & 100,0 \\
\hline Sonstige & 3 & \\
\hline Gesamt & 3 & \\
\hline
\end{tabular}

Anmerkung: Graue Unterlegung der konfessionell homogenen Gruppen I* s. Fußnote 15 I** s. Fußnote 16 I Nicht-religiöse MSO $\left(n_{S}\right)=150$; Religiöse MSO $\left(n_{R}\right)=78$.

${ }^{15} \mathrm{Zu}$ den genannten Glaubensrichtungen, denen alle Mitglieder angehören, gehören in jeweils 4 MSO muslimisch und evangelisch-freikirchlich, in 3 Vereinigungen griechischorthodox und in jeweils 2 Gemeinden christlich-orthodox, orthodox-katholisch, evangelisch, ezidisch, jüdisch-liberal. Einzelne Anführungen nennen buddhistisch, hinduistisch sowie syrisch-orthodox.

${ }^{16} \mathrm{Zu}$ den in 29 MSO als dominant vertreten genannten Glaubensrichtungen gehören griechisch-orthodox (5 MSO), orthodox-katholisch (4 MSO), evangelisch (3 MSO), evangelisch-freikirchlich, hinduistisch, ezidisch, muslimisch, pfingstlich-charismatisch, schiitisch (jeweils 2 MSO) sowie in jeweils einer MSO buddhistisch, bulgarisch-orthodox, jüdisch-liberal, jüdisch-orthodox und russisch-orthodox. 
fehlende als auch „Weiß nicht“- sowie Angaben unter „Sonstiges“ gefasst wurden, die keine Auskunft zu Konfessionen gaben, sondern kommentierten, dass Religion in der MSO keine Rolle spiele.

Eine Kategorisierung der migrantischen Organisationen in Bezug auf eine für „alle“ Mitglieder angegebene gleiche Konfessionszugehörigkeit zeigt, dass in 38 Prozent der MSO eine konfessionelle Homogenität und in 61 Prozent hinsichtlich der Glaubensorientierungen der Mitglieder eine Heterogenität festzustellen ist (Tab. 7.9). Letztere liegt - wie oben und in der Tab. 7.8 dargelegt - allerdings in deutlich unterschiedlichen Ausmaßen vor.

Tab. 7.9 Konfessionelle Homogenität und Heterogenität der Mitglieder

\begin{tabular}{lrc}
\hline & Häufigkeit & Prozent \\
\hline Homogenität & 87 & 38,2 \\
\hline Heterogenität & 140 & 61,4 \\
\hline Alle konfessionslos & 1 & 0,4 \\
\hline Gesamt & 228 & 100,0 \\
\hline $\mathrm{n}_{\mathrm{S}}=150 ; \mathrm{n}_{\mathrm{R}}=78$ & &
\end{tabular}

Spiegelt man diesen Befund mit der Zuordnung der MSO zu einer religiösen Tradition, dann lässt sich für 57 der religiösen MSO (73\%) eine konfessionelle Homogenität feststellen. Diese bezieht sich in 35 der religiösen MSO (45\%) auf den Islam (,alle sunnitisch“ bzw. ,alle muslimisch“). In 7 von den 12 sich dem Alevitentum zuordnenden MSO sind alle Mitglieder alevitisch. Ebenso sind die Mitglieder in 4 der 5 den orthodoxen Kirchen zuzurechnenden religiösen MSO hinsichtlich der Glaubensrichtung homogen. Eine jeweils gleiche Konfessionszugehörigkeit der Mitglieder ist zudem in 6 der insgesamt 10 christlichen MSO festzustellen (,,alle evangelisch-freikirchlich“, ,,alle evangelisch“, ,,alle römischkatholisch"). Einheitliche Religionszugehörigkeiten der Mitglieder sind zudem in 2 ezidischen und zwei jüdischen MSO auszumachen. In der hinduistischen MSO sind alle Mitglieder hinduistisch.

Es zeigt sich, dass nach Einschätzung der Funktionsträger in 30 der nichtreligiösen MSO (20\%) alle Mitglieder einer einzigen Glaubensrichtung angehören. Dazu gehören mehrheitlich das Sunnitentum (11\%), gefolgt von Einzelfällen wie dem römischen Katholizismus (3\%), der christlichen Orthodoxie (3\%) sowie dem Alevitentum, dem Buddhismus sowie dem Evangelium in jeweils einer MSO (1\%). 
Analog zur Ermittlung der Herkunftsländer spiegeln die Ergebnisse zum einen eine insbesondere für religiöse MSO zu erwartende konfessionelle Homogenität der Mitglieder, zum anderen aber auch eine Mischung unterschiedlicher Glaubensrichtungen. Die Homogenität scheint sinnfällig und in der Natur der Sache zu liegen, da sich Personen mit gleichen religiösen Anschauungen zusammentun, um gemeinschaftlich religiöse Interessen und Zwecke zu verfolgen. Aufgrund dieses Selektionseffektes dürften Mitgliedschaften in Vereinigungen, die bestimmten Konfessionen zugehörig sind, seitens nicht-religiöser oder andersgläubiger Personen nicht oder in deutlich geringerem Ausmaß in Betracht gezogen werden (II. 4.4.2). Angesichts derErgebnisse verbieten sich jedoch pauschale Annahmen, dass MSO mit einer religiösen Tradition per se eine in Bezug auf die Glaubensrichtung homogene Mitgliederstruktur aufweisen, während das bei säkularen migrantischen Vereinigungen nicht der Fall sein sollte. Die Befunde lassen sich dahingehend deuten, dass Religion bzw. eine Affinität zu religiösen Glaubensrichtungen auch in „säkularen“ MSO, in denen eine konfessionelle Gleichheit der Mitglieder vorzufinden ist, eine Rolle spielen und daher auch für den Zusammenschluss bzw. die Entscheidung für die Mitgliedschaft bedeutsam sein könnten. Zudem weisen die in den MSO auszumachenden konfessionell durchmischten Binnenleben darauf hin, dass auch in den sich offiziell keiner religiösen Tradition zuordnenden Vereinigungen Religionszugehörigkeiten wahrgenommen werden und von Bedeutung sein können, ohne jedoch (vermutlich) innerhalb der Organisationen eine bestimmende Rolle zu spielen. Weiter gedacht könnte dies bedeuten, dass in den Vereinigungen statt Differenzorientierungen potenziell Perspektivenübernahmen eingeübt werden können. Denn Binnen- und Außenperspektiven auf eine spezifische Religion treffen aufeinander, was Auseinandersetzungen mit anderen Religionen, Wertevorstellungen und auch Religionslosigkeit stimulieren kann. Dies sind wichtige Voraussetzungen für eine Pluralismusbefähigung und ein respektvolles Zusammenleben von Menschen mit differenten (nicht-)religiösen Lebensformen.

\section{Mitgliedschaftsvoraussetzungen: Für 41 Prozent der MSO relevant}

Die Filterfrage nach der Existenz von mit der Mitgliedschaft verknüpften Voraussetzungen bejahten 41 Prozent der MSO, davon 49 Prozent der religiösen und 37 Prozent der nicht-religiösen MSO. In der darauffolgenden Frage sollten die Bedingungen konkretisiert werden; es waren mehrere Antworten möglich. Die Empfehlung von Mitgliedern (35\%) wurde am häufigsten und öfters als die Zugehörigkeit zu einer bestimmten Glaubensgemeinschaft (29\%) genannt (Abb. 7.3). Letzterer wurde von 64 Prozent der religiösen MSO $\left(n_{R}=39\right)$ und 8 Prozent der säkularen $\left(n_{S}=65\right)$ zugestimmt. Die Empfehlungen von Mitgliedern sind mit 42 Prozent für die nicht-religiösen Organisationen relevanter (vs. $23 \%$ ). 


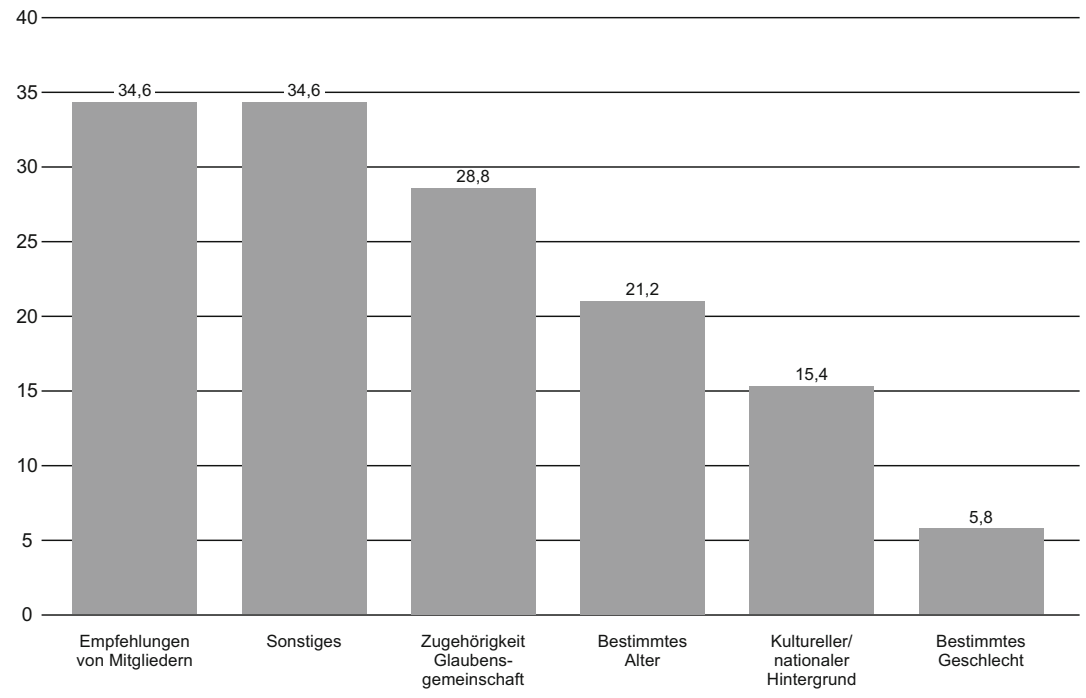

$n=104$

Abb. 7.3 Nennung von Mitgliedschaftsvoraussetzungen (in \%; Mehrfachnennung)

Ein bestimmtes Alter wurde von 21 Prozent der MSO und ein bestimmter kultureller bzw. nationaler Hintergrund von 15 Prozent der Befragten markiert. Bezüglich des Alters zeigt sich hier unter den religiösen mit 28 Prozent eine höhere Bejahungsrate als bei den säkularen Organisationen (17\%). Letztere geben jedoch häufiger an, dass eine bestimmte Nationalität eine Mitgliedschaftsbedingung darstellt (20\% vs. $8 \%$ ). Der Zugehörigkeit zu einem bestimmten Geschlecht kommt insgesamt nur eine marginale Rolle (6\%) zu.

Des Weiteren wurden für 35 Prozent der MSO - und damit gleichauf mit den Empfehlungen von Mitgliedern - unter „Sonstiges“ weitere Mitgliedschaftsvoraussetzungen vermerkt. Darunter fallen überwiegend bestimmte Wertorientierungen und politische Einstellungen (12\%), wie etwa die Befürwortung von demokratischen, liberalen und weltoffenen Werten, individuelle Vorurteilsfreiheit gegenüber anderen Menschen oder die Anhängerschaft von Mustafa Kemal Atatürk. Die genannten Wertemaßstäbe sind sowohl für säkulare als auch nichtreligiöse MSO relevant. Darüber hinaus wurden als permissivere Bedingungen die Akzeptanz der Satzung bzw. des Leitbildes des Vereins (7 \%) und das Vorhandensein spezifischer Interessen benannt (7 \%), z. B. an der polnischen, französischen 
oder kurdischen Kultur oder Literatur. Auch wurde das Kriterium bestimmter Qualifikationen bzw. Nachweise (6 \%) wie etwa der Besitz eines Hochschulabschlusses, eines Sportlerpasses oder Führungszeugnisses oder Kenntnisse in der Wissensvermittlung und Veranstaltungsorganisation angeführt.

Eine Betrachtung der genannten Mitgliedschaftsvoraussetzungen in der Summe zeigt, dass 66 Prozent der MSO eine Voraussetzung, 26 Prozent zwei Bedingungen, 6 Prozent drei und 2 Prozent vier Kriterien an die Mitgliedschaft knüpfen. Ein Vergleich zwischen religiösen und säkularen MSO zeigt, dass letztere häufiger eine Bedingung nennen (MO: $71 \%$ vs. RMO: $59 \%$ ), während erstere öfters zwei Erwartungen anführen (RMO: $36 \%$ vs. MO: $20 \%$ ).

In einem separaten Auswertungskapitel (7.2.3) wird näher beleuchtet, inwieweit die Mitgliedschaftsvoraussetzungen in Kombination mit der Angabe von Zielgruppen und dem Kooperationsverhalten der MSO auf die Absicht der Stärkung des Zusammenhalts der eigenen Gruppe und Abgrenzungstendenzen (,,bonding“) schließen lassen.

\subsubsection{Räumlichkeiten sowie finanzielle Quellen und Ressourcen}

Räumlichkeiten: Religiöse MSO verfügen signifikant häufiger über Lokalitäten

76 Prozent der MSO geben an, Räumlichkeiten nutzen zu können, wobei dies für 89 Prozent der religiösen im Vergleich zu 70 Prozent der nicht-religiösen Vereinigungen häufiger zutrifft. Zwischen den beiden kategorialen Variablen „Räumlichkeiten“ / „Religiöse Tradition“ (nein/ja) kann ein signifikanter (schwacher) Zusammenhang ermittelt werden (Cramers $V=0,204 * * *)$ : Den religiösen MSO stehen häufiger Räumlichkeiten zur Verfügung.

\section{Finanzielle Quellen}

Bei der Frage, wie sich die MSO überwiegend finanzieren, waren 10 Items (inkl. „Sonstiges“) vorgegeben und mehrfache Antworten möglich. 76 Prozent der MSO finanzieren sich überwiegend über Mitgliedsbeiträge, 67 Prozent über Spenden und Sponsorengelder, 30 Prozent erhalten Fördermittel der Stadt, 25 Prozent beziehen Fördermittel des Landes Nordrhein-Westfalen und 14 Prozent vom Bund. Lediglich 5 Prozent der MSO erhalten Zuschüsse von Dachverbänden, 2 Prozent Unterstützung durch Kirchen und 2 Prozent durch Wohlfahrtsverbände. Nur eine einzige Organisation gab an, finanzielle Unterstützung durch Organisation(en) aus dem Ausland zu erhalten. 
17 Prozent teilten auf dem Fragebogen nicht vorgegebene Finanzierungsquellen mit. Hierunter fallen eigene Einnahmen bzw. Selbsterwirtschaftung (9 \%), z. B. über eigene Veranstaltungen oder die Erhebung von Kursgebühren. Des Weiteren wurden Projekt- $(4 \%)$ und Stiftungsgelder (3\%) sowie Auftragsarbeit (z. B. vom Jugendamt) (1\%) angeführt.

Betrachtet man die unterschiedlichen finanziellen Ressourcen separiert nach religiösen und nicht-religiösen MSO, so zeigen sich folgende erwähnenswerte Unterschiede (Abb. 7.4): Spenden und Sponsorengelder empfangen 80 Prozent der religiösen und 61 Prozent der nicht-religiösen Organisationen. Fördermittel der Stadt erreichen 40 Prozent der säkularen und mit 10 Prozent eine deutlich geringere Anzahl der religiösen Vereinigungen. Eine deckungsgleiche Diskrepanz zeigt sich hinsichtlich des Bezugs von Fördermitteln durch das Land Nordrhein-Westfalen: Hier steht ein geförderter Anteil von 35 Prozent der nicht-religiösen einem Anteil von lediglich 5 Prozent der religiösen Organisationen gegenüber. Und während letztere überhaupt keine Unterstützung durch Fördermittel oder -programme des Bundes erhalten, trifft dies für 21 Prozent der nicht-religiösen zu.

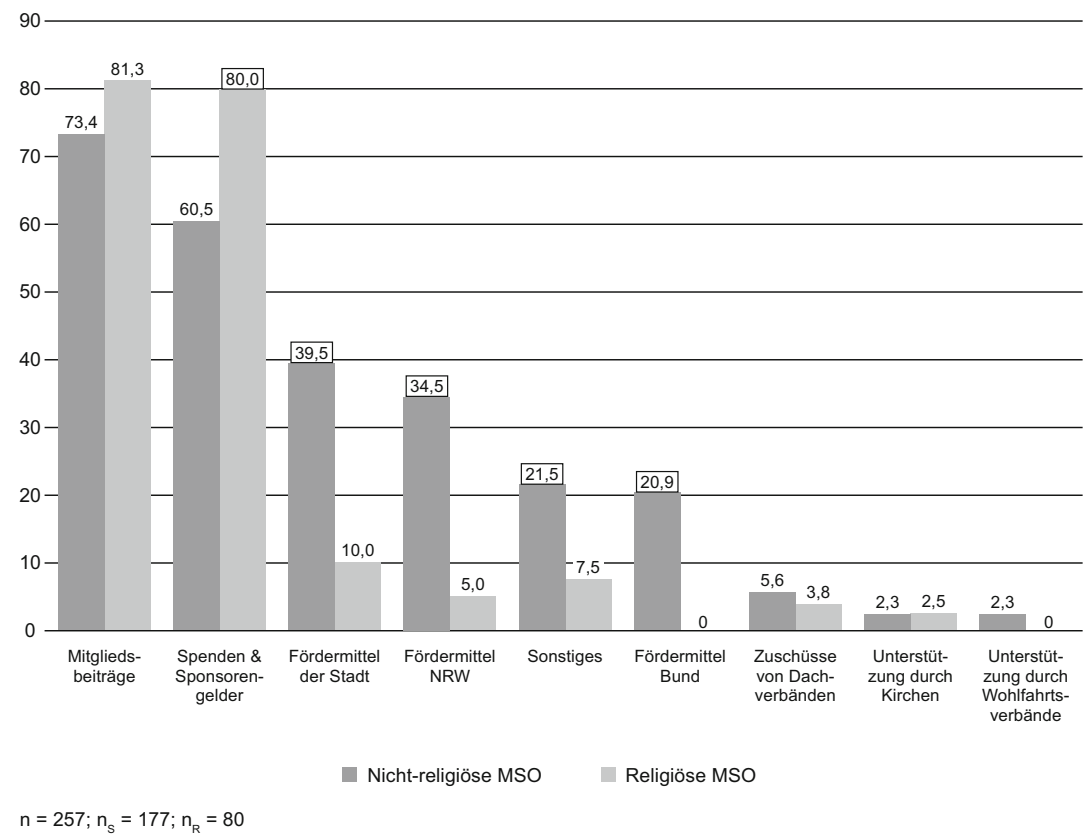

Abb. 7.4 Finanzierungsquellen der MSO (in \%) 
Religiöse MSO: Mit weniger öffentlichen Finanzierungsquellen vermögender

Hinsichtlich der Spenden und Sponsorengelder zeigt sich eine signifikant gröBere Bedeutung für die religiösen MSO. Fördermittel von Stadt und Bund sowie insbesondere des Landes Nordrhein-Westfalen stellen hingegen für die säkularen Vereinigungen gewichtigere Subventionen dar. ${ }^{17}$

Eine Aufsummierung der pro Organisation angegebenen Finanzierungsquellen verdeutlicht, dass die meisten der MSO (41\%) über zwei Instanzen, und zwar Mitgliedsbeiträge und Spenden/Sponsorengelder, Geld beziehen und 23 Prozent zumeist über eine Quelle, die ebenfalls Mitgliedsbeiträge oder Spenden/Sponsorengelder betrifft. 17 Prozent erhalten über drei Einrichtungen finanzielle Mittel (Tab. 7.10). Unter den MSO, die ihre Kosten überwiegend über zwei Quellen begleichen, befinden sich mit 58 Prozent anteilig mehr religiöse MSO (+24 Prozentpunkte), die bis auf eine Ausnahme generell auch nicht mehr als drei Instanzen in Anspruch nehmen (Tab. 7.10).

Für die Prüfung, ob zwischen den religiösen und säkularen MSO hinsichtlich der Anzahl der Finanzierungsquellen ein signifikanter Unterschied vorliegt, wurde der $t$-Test für zwei unabhängige Stichproben durchgeführt. Dieser zeigt, dass säkulare MSO auf eine größere Anzahl von Finanzierungsquellen zurückgreifen können. ${ }^{18}$

Tab.7.10 Anzahl von Finanzierungsquellen für nicht-religiöse und religiöse MSO

\begin{tabular}{crrrrrrr}
\hline $\begin{array}{c}\text { Anzahl der } \\
\text { Quellen }\end{array}$ & Häufigkeit & in $\%$ & Nicht-religiöse MSO & \multicolumn{2}{c}{ Religiöse MSO } \\
\hline 1 & 60 & 23,3 & 38 & {$[21,5 \%]$} & 22 & {$[27,5 \%]$} \\
\hline 2 & 106 & 41,2 & 60 & {$[33,9 \%]$} & 46 & {$[57,5 \%]$} \\
\hline 3 & 44 & 17,1 & 33 & {$[18,6 \%]$} & 11 & {$[13,8 \%]$} \\
\hline 4 & 26 & 10,1 & 26 & {$[14,7 \%]$} & - & \\
\hline 5 & 13 & 5,1 & 12 & {$[6,8 \%]$} & 1 & {$[1,3 \%]$} \\
\hline 6 & 7 & 2,7 & 7 & {$[4,0 \%]$} & - & \\
\hline 7 & 1 & 0,4 & 1 & {$[0,6 \%]$} & - & \\
\hline Gesamt & 257 & 100,0 & 177 & {$[100,0 \%]$} & 20 & {$[100,0 \%]$} \\
\hline
\end{tabular}

${ }^{17}$ Spenden und Sponsorengelder (Cramers $\left.V=0,192^{* *}\right)$; Fördermittel der Stadt $(V=$ $\left.0,298^{* * *}\right)$; Fördermittel des Bundes $\left(V=0,276^{* * *}\right)$; Fördermittel des Landes NordrheinWestfalen $(V=0,314 * * *)$.

${ }^{18}$ Varianzhomogenität liegt mit $F=47,305 ; p<0,001$ nicht vor. Im $t$-Test mit Welch-Korrektur wird der Gruppenunterschied signifikant. Nicht-religiöse Gruppe: $M=2,66 ; S D=1,398$; $\mathrm{n}_{\mathrm{S}}=177$; religiöse Gruppe: $M=1,9 ; S D=0,722 ; \mathrm{n}_{\mathrm{R}}=80 \mid t(250,579)=5,7 ; p=0,000$; Cohen's $d=0,62$. 
Die Ergebnisse lassen sich einerseits dahingehend interpretieren, dass sie die Präferenzen politischer Förderungen von MSO auf Bundes-, Landes- und kommunaler Ebene in NRW widerspiegeln. Wenngleich - im Gegensatz zu bundespolitischen Verlautbarungen - für das Bundesland Nordrhein-Westfalen und seine Kommunen eine Förderung von religiösen migrantischen Vereinigungen nicht explizit abgelehnt wird und eine integrative Ausrichtung der MSO für deren Unterstützung ausschlaggebend ist ${ }^{19}$ (LT NRW 2018: 106), ist mit Blick auf registrierte Radikalisierungstendenzen religiöser MSO sowie anhaltender, nicht tolerierter politischer Einflussnahmen aus dem Ausland auf zunehmende Verunsicherung und Skepsis zu schließen (ebd.). In diesem Zusammenhang werden Grundgesetzkonformität bzw. die Akzeptanz der freiheitlich-demokratischen Verfassung, integrative Aktivitäten sowie die Erfüllung seitens politischer Akteure formulierter Forderungen, die u. a. die Unabhängigkeit von Herkunftsländern betreffen, zu zentralen Voraussetzungen für den Bezug finanzieller Subventionen erklärt (s. hierzu auch Klie 2020; II. 3.2; II. 4.3.5).

Andererseits könnte es wiederum auch sein, dass bei religiösen Vereinigungen der Wunsch nach bzw. Bedarf an politischen Fördermitteln weniger stark ausgeprägt ist und entsprechend wenig(er) Förderanträge gestellt werden. Zudem ist davon auszugehen, dass MSO mit hauptamtlichem Personal und einer fachlichen Professionalisierung, die bei den nicht-religiösen MSO ausgeprägter ist (7.1.2.3), bei der Akquise von Fördermitteln einen strukturellen Vorteil besitzen (vgl. Friedrichs et al. 2020: 75 f.).

\section{Finanzvolumen pro Jahr}

Die Frage nach dem einer Organisation pro Jahr zur Verfügung stehenden Finanzvolumen durfte geschätzt werden. Die Antworten legen dar, dass die meisten MSO (31\%) über weniger als 10.000 Euro und 28 Prozent über 10.000 bis 50.000 Euro verfügen. Mit weniger als 2.000 Euro müssen 18 Prozent der MSO auskommen (Tab. 7.11). Im Vergleich dazu standen der Studie des SVR-Forschungsbereichs zufolge 38 Prozent der befragten MSO im Jahr 2019 10.000 Euro und 13 Prozent 1.000 Euro zur Verfügung. Auch in der SVR-Studie gaben einzelne MSO (3\%) an, über eine Million Euro zu verfügen (Friedrichs et al. 2020: 69; I. 2.1).

19 „Wir dulden keine Hassprediger und keine Einflussnahme fremder Staaten. Die Zusammenarbeit mit religiösen Verbänden wird sich danach richten, inwiefern sie Integration fördern oder behindern“ (LT NRW 2018: 106). 
Tab. 7.11 Finanzvolumen pro Jahr

\begin{tabular}{lcc}
\hline Finanzvolumen/Jahr & Häufigkeit & Prozent \\
\hline Weniger als 2.000 Euro & 44 & 18,2 \\
\hline Weniger als 10.000 Euro & 75 & 31,0 \\
\hline 10.000 bis 50.000 Euro & 68 & 28,1 \\
\hline 50.000 bis 100.000 Euro & 25 & 10,3 \\
\hline Mehr als 100.000 Euro & 20 & 8,3 \\
\hline Mehr als 500.000 Euro & 6 & 2,5 \\
\hline Mehr als 1 Million Euro & 3 & 1,2 \\
\hline Sonstiges (= „gar keins“) & 1 & 0,4 \\
\hline Gesamt & 242 & 100,0 \\
\hline
\end{tabular}

Mitgliederreiche und religiöse MSO sind signifikant finanzstärker

Die Ergebnisse spiegeln die an anderer Stelle dieser Arbeit bereits thematisierte heterogene Ressourcenausstattung von kleinen und großen sowie gar nicht oder stark professionalisierten MSO wider (II. 4.3.2). Die Berechnung des Rangkorrelationskoeffizienten nach Spearman ermittelt für die beiden ordinalskalierten Variablen der Mitgliederanzahl sowie des Finanzvolumens einen signifikanten positiven Zusammenhang mittleren Effektes $\left(r h o=0,35^{* * *}\right)$ : Je mehr Mitglieder eine MSO hat, desto mehr Geld steht ihr pro Jahr zur Verfügung.

Für religiöse und säkulare MSO zeigen sich Unterschiede. Während letztere deutlich häufiger über weniger als 2.000 oder 10.000 Euro verfügen, tun dies die religiösen Vereinigungen in den gestaffelten Summen ab 10.000 Euro (Abb. 7.5).

Die Berechnung des $U$-Tests zeigt, dass sich religiöse und säkulare MSO in Bezug auf das ihnen per Anno zur Verfügung stehende Finanzvolumen in ihren Zentralwerten signifikant unterscheiden und dass die befragten religiösen Vereinigungen größere Kapitalien besitzen. ${ }^{20}$

Die zentrale Frage, ob ein vorhandenes Finanzvolumen für die Begleichung laufender und darüber hinausgehend anfallender Kosten ausreicht, ist nur fallspezifisch und von den MSO selbst zu beantworten. 46 Prozent der MSO geben an, finanzielle Engpässe zu haben, 54 Prozent verneinen dies. Unter letzteren sind mit 62 Prozent häufiger religiöse Vereinigungen vertreten (vs. MO: $51 \%$ ). Zwischen den beiden kategorialen Variablen „Religiöse Tradition“ / „Finanzielle Engpässe" (nein/ja) besteht kein signifikanter Zusammenhang (Cramers $V=0,106, p=$ 0,091).

${ }^{20} \underline{U}$-Test: $U=4581 ; z=-3,13 ; p=0,002 ; r=0,2 ; \mathrm{Md}_{\mathrm{S}}=2 ; \mathrm{Md}_{\mathrm{R}}=3$. 


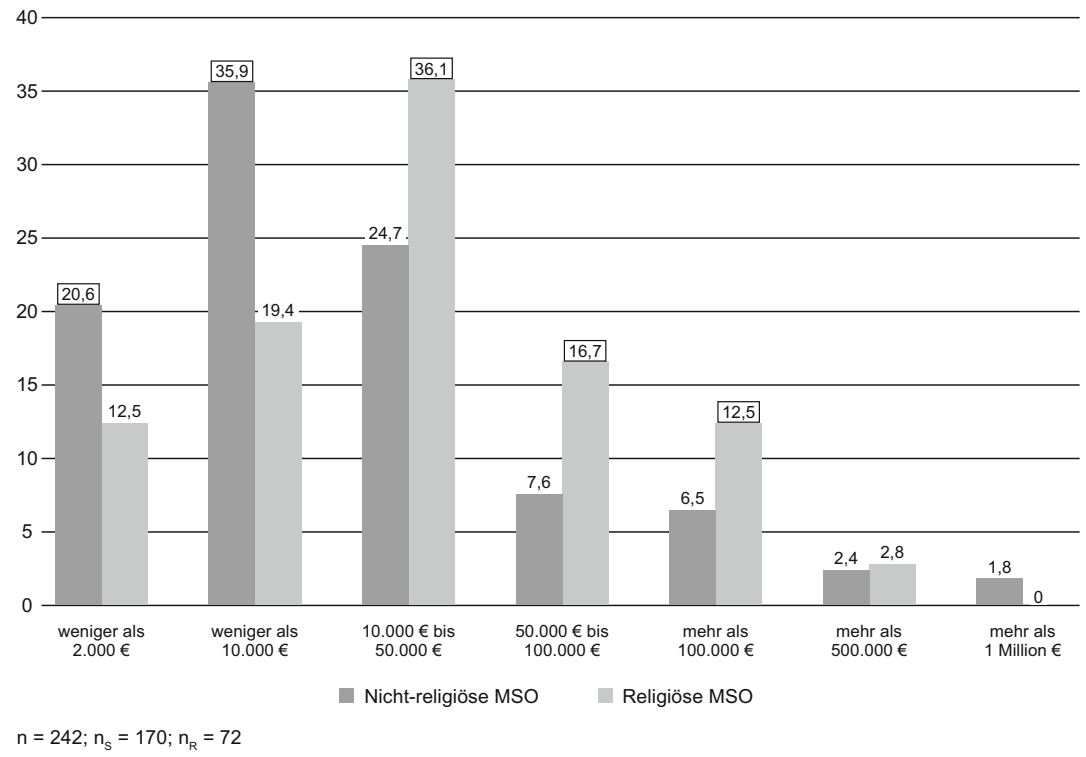

Abb. 7.5 Finanzvolumen pro Jahr für nicht-religiöse und religiöse MSO (in \%)

Eine bivariate Tabellierung der Finanzvolumina per Anno mit den Fällen konstatierter finanzieller Mängel zeigt, dass 22 Prozent letzterer über weniger als 2.000 Euro, 32 Prozent über weniger als 10.000 Euro, 32 Prozent über 10.000 bis 50.000 Euro und 11 Prozent über 50.000 bis 100.000 Euro verfügen. Es besteht ein schwacher, aber signifikanter Zusammenhang zwischen den beiden Variablen „Finanzvolumen pro Jahr“ und ,finanzielle Engpässe“ (rho $=-0,16 *$; Berechnung der biserialen Rangkorrelation).

\subsubsection{Personelle Ressourcen: Hauptamtliche und Engagierte}

Hauptamtliche: Säkulare MSO haben mehr

33 Prozent der MSO geben an, hauptamtliche, bezahlte Mitarbeiter zu haben. Mit Blick auf die religiösen MSO zeigt sich, dass diese mit 41 Prozent gegenüber 30 Prozent der nicht-religiösen Vereinigungen häufiger auf Hauptamtliche zurückgreifen können. 
Hauptamtliche sind in den meisten MSO (48 \%) mit einer Anzahl von 2 bis 10 Personen und mit einer Person in 35 Prozent der Vereinigungen vertreten (Tab. 7.12). Die säkularen MSO verfügen über höhere und vereinzelt sehr hohe Zahlen an Hauptamtlichen. Die Berechnung des $U$-Tests zeigt, dass sie über signifikant mehr Hauptamtliche verfügen. ${ }^{21}$

Tab. 7.12 Anzahl von Hauptamtlichen in nicht-religiösen und religiösen MSO (in absoluten und relativen Häufigkeiten)

\begin{tabular}{|c|c|c|c|c|}
\hline $\begin{array}{l}\text { Haupt- } \\
\text { amtliche }\end{array}$ & $\begin{array}{l}\text { Häufigkeit } \\
\text { alle MSO }\end{array}$ & $\begin{array}{l}\text { Alle MSO } \\
\text { (in \%) }\end{array}$ & $\begin{array}{l}\text { Nicht-religiöse MSO } \\
\text { [in \%] }\end{array}$ & $\begin{array}{c}\text { Religiöse MSO } \\
\text { [in \%] }\end{array}$ \\
\hline 1 & 29 & 34,5 & $10[19,6]$ & $19 \quad[57,6]$ \\
\hline 2 bis 10 & 40 & 47,6 & $27 \quad[52,9]$ & $13 \quad[39,4]$ \\
\hline 11 bis 20 & 6 & 7,1 & {$[9,8]$} & {$[3,0]$} \\
\hline 21 bis 30 & 3 & 3,6 & {$[5,9]$} & - \\
\hline 31 bis 40 & 2 & 2,4 & {$[3,9]$} & - \\
\hline 50 & 2 & 2,4 & {$[3,9]$} & - \\
\hline 196 & 1 & 1,2 & {$[2,0]$} & - \\
\hline 250 & 1 & 1,2 & {$[2,0]$} & - \\
\hline Gesamt & 84 & 100,0 & $51[100,0]$ & $33[100,0]$ \\
\hline
\end{tabular}

Das Vorhandensein von bezahlten Hauptamtlichen verweist auf den Spezialisierungs- und Formalisierungsgrad der Organisationen, d. h. die Ausdifferenzierung spezifischer Aufgaben und ihre Zuordnung zu bestimmten Funktionen und Rollen, die mit spezifischen Erwartungen und Qualifikationen verknüpft sind (II. 4.3.4). Je höher die Anzahl der Hauptamtlichen ausfällt, desto spezialisierter, professioneller und formalisierter sind die MSO, was insbesondere für die säkularen zutrifft.

Zudem wird deutlich, dass einige hochprofessionelle Organisationen an der Befragung teilgenommen haben, in denen es gar keine Engagierten gibt. Zwei Einzelfälle mit 196 und 250 Hauptamtlichen wie auch neun weitere Organisationen mit 50, 29 und 1 bis 10 Hauptamtlichen verfügen über gar keine Engagierten.

Die Prüfung, ob ein Zusammenhang zwischen der Anzahl der Hauptamtlichen und der Anzahl der Engagierten besteht, ergibt eine nicht signifikante schwache, negative Korrelation nach Spearman $(\text { rho }=-0,14 ; p=0,257)^{22}$.

${ }^{21} U$-Test: $U=448 ; z=-3,68 ; p=0,000 ; r=0,4 ; \mathrm{Md}_{\mathrm{S}}=5 ; \mathrm{Md}_{\mathrm{R}}=1$. Durch die Rangbildung verschwindet der Effekt von einflussreichen Fällen (,Ausreißern“).

22 Da die Variablen mit Ausreißern behaftet sind, kann die robuste Spearman-Korrelation angewendet werden. 
Freiwillig Engagierte: Religiöse MSO haben signifikant mehr

Für nicht gewinnorientierte Organisationen stellen die sich freiwillig und unbezahlt in die Organisation einbringenden Personen eine Hauptressource dar, da diese durch ihren persönlichen Einsatz den Status und die Existenz der Organisationen sichern. Aufgrund des Aspekts der Freiwilligkeit einer Tätigkeitsübernahme ist davon auszugehen, dass der Formalisierungsgrad der Anforderungen im Vergleich zu haupt- oder ehrenamtlichen Ämtern und Positionen gering(er) ausfällt (II. 4.3.4).

240 der befragten Funktionsträger (93\%) gaben an, dass in ihrer Organisation Personen freiwillig und unbezahlt bestimmte Aufgaben übernehmen. ${ }^{23}$ Die Anzahl von Engagierten kumuliert mit 56 Prozent deutlich bei 1 bis 10 Personen (Tab. 7.13).

Tab.7.13 Anzahl von Engagierten in nicht-religiösen und religiösen MSO (in absoluten und relativen Häufigkeiten)

\begin{tabular}{|c|c|c|c|c|c|c|}
\hline \multirow{2}{*}{$\begin{array}{l}\text { Engagierte } \\
1 \text { bis } 10\end{array}$} & \multirow{2}{*}{$\begin{array}{c}\begin{array}{c}\text { Häufigkeit } \\
\text { alle MSO }\end{array} \\
133\end{array}$} & \multirow{2}{*}{$\begin{array}{c}\begin{array}{c}\text { Alle MSO } \\
\text { (in \%) }\end{array} \\
55,9\end{array}$} & \multicolumn{2}{|c|}{$\begin{array}{c}\text { Nicht-religiöse MSO } \\
\text { [in \%] }\end{array}$} & \multicolumn{2}{|c|}{$\begin{array}{c}\text { Religiöse MSO } \\
\text { [in \%] }\end{array}$} \\
\hline & & & 110 & {$[67,9]$} & 23 & {$[30,3]$} \\
\hline 11 bis 20 & 57 & 23,9 & 23 & {$[19,8]$} & 25 & {$[32,9]$} \\
\hline 21 bis 30 & 14 & 5,9 & 7 & {$[4,3]$} & 7 & {$[9,2]$} \\
\hline 31 bis 40 & 6 & 2,5 & 2 & {$[1,2]$} & 4 & {$[5,3]$} \\
\hline 41 bis 50 & 14 & 5,9 & 7 & {$[4,3]$} & 7 & {$[9,2]$} \\
\hline 60 & 3 & 1,3 & 1 & {$[0,6]$} & 2 & {$[2,6]$} \\
\hline 90 & 2 & 0,8 & 1 & {$[0,6]$} & 1 & {$[1,3]$} \\
\hline 120 bis 150 & 3 & 1,3 & 1 & {$[0,6]$} & 2 & {$[2,6]$} \\
\hline 200 & 2 & 0,8 & - & & 2 & {$[2,6]$} \\
\hline 300 & 1 & 0,4 & - & & 1 & {$[1,3]$} \\
\hline 500 & 1 & 0,4 & - & & 1 & {$[1,3]$} \\
\hline 1.000 & 1 & 0,4 & 1 & {$[0,6]$} & - & \\
\hline 16.500 & 1 & 0,4 & - & & 1 & {$[1,3]$} \\
\hline Gesamt & 238 & 100,0 & 162 & {$[100,0]$} & 76 & {$[100,0]$} \\
\hline
\end{tabular}

In einzelnen MSO fallen die Engagiertenzahlen sehr hoch aus und sind insbesondere religiösen Organisationen mit über 100 Mitgliedern zuzuordnen (Tab. 7.13; graue Unterlegungen). Die Zahl 16.500 wurde von einem dem Bund der Alevitischen Jugendlichen in Deutschland e. V. (BDAJ) zugehörigen

\footnotetext{
${ }^{23}$ Es handelt sich um eine Filterfrage. Für die MSO ohne engagierte Personen waren die darauffolgenden fünf engagementbezogenen Fragen nicht zu beantworten.
} 
Verein angegeben, mit einem handschriftlichen Vermerk dahinter: „Alle sind ehrenamtlich tätig." Eine Mennoniten-Brüdergemeinde zählt 500, eine DİTİBGemeinde 300, eine islamische und eine VIKZ-Gemeinde geben jeweils 200 und eine evangelisch-freikirchliche und islamische Vereinigung jeweils 120 bis 150 freiwillig und unbezahlt Tätige an.

Ein Vergleich zwischen der Verteilung der Engagiertenzahlen auf nichtreligiöse und religiöse MSO zeigt, dass 68 Prozent der nicht-religiösen gegenüber 30 Prozent der religiösen Organisationen 1 bis 10 Freiwillige haben. Hingegen verfügen 33 Prozent der religiösen im Vergleich zu 20 Prozent der nicht-religiösen Vereinigungen häufiger über 11 bis 20 Engagierte. Auch in den nächstfolgenden Gruppen (21 bis 30, 31 bis 40, 41 bis 50) sind die religiösen Organisationen mit höheren Prozentwerten als die nicht-religiösen vertreten (Tab. 7.13). Anhand der Berechnung des $U$-Tests zeigt sich, dass die religiösen MSO über signifikant mehr Engagierte verfügen. ${ }^{24}$

Dieses Phänomen könnte mit den dichten Gemeindestrukturen der religiösen MSO und dort vermehrt stattfindenden Interaktionen $\mathrm{zu}$ erklären sein, die es in besonderem Maße ermöglichen, viele Freiwillige zu gewinnen (II. 4.4.2). Auch liegt die Vermutung nahe, dass die religiösen Vereinigungen aufgrund ihrer Abhängigkeit von Engagierten aktiv bestrebt sind, solche zu gewinnen und im Vergleich zu den säkularen MSO dadurch erfolgreicher sind. Diese in einer Hypothese $\left(\mathrm{H}_{1}\right)$ formulierten Annahme bedarf im weiteren Verlauf der Auswertung einer gesonderten Überprüfung.

Proportionales Verhältnis zwischen Mitglieder- und Engagiertenzahlen

Ein weiteres Interesse besteht darin festzustellen, wie sich die Engagierten- zu den Mitgliederzahlen verhalten. Anhand einer bivariaten Tabellierung der gruppierten Engagierten- mit den Mitgliederzahlen sind proportionale Tendenzen ersichtlich (Tab. 7.14). Die in insgesamt 56 Prozent der MSO vorhandenen 1 bis 10 Engagierten sind v. a. in Organisationen mit 11 bis 50 Mitgliedern, mit sprunghaft sinkenden Zahlen aber auch in den mitgliederreicheren MSO vorzufinden (Tab. 7.14). Mit Blick auf die Verteilung von 1 bis 10 Freiwilligen auf die unterschiedlich mitgliederstarken MSO zeigt sich eine interessante Parallelerscheinung: Sowohl in 19 MSO mit weniger als 10 Mitgliedern als auch 17 der Organisationen mit mehr als 100 Mitgliedern sind 1 bis 10 Engagierte zuzuordnen.

${ }^{24} U$-Test: $U=3243 ; z=-5,92 ; p=0,000 ; r=0,4 ; \operatorname{Md}_{\mathrm{S}}=10 ; \mathrm{Md}_{\mathrm{R}}=20$. Durch die Rangbildung verschwindet der Effekt von einflussreichen Fällen (,Ausreißern“). 
Tab. 7.14 Engagierte nach Anzahl der Mitglieder in MSO (in absoluten und relativen Häufigkeiten)

\begin{tabular}{lcccccc}
\hline Engagierte & \multicolumn{7}{c}{ Anzahl Mitglieder } \\
& unter $\mathbf{1 0}$ & $\mathbf{1 1}$ bis $\mathbf{5 0}$ & $\mathbf{5 1}$ bis $\mathbf{1 0 0}$ & mehr als $\mathbf{1 0 0}$ & Gesamt \\
\hline 1 bis 10 & 19 & 58 & 38 & 17 & 132 & {$[55,7 \%]$} \\
\hline 11 bis 20 & 1 & 9 & 19 & 28 & 57 & {$[24,1 \%]$} \\
\hline 21 bis 30 & - & 4 & 2 & 8 & 14 & {$[5,9 \%]$} \\
\hline 31 bis 40 & - & 3 & - & 3 & 6 & {$[2,5 \%]$} \\
\hline 41 bis 50 & - & 2 & 1 & 11 & 14 & {$[5,9 \%]$} \\
\hline 60 & - & - & 2 & 1 & 3 & {$[1,3 \%]$} \\
\hline 90 & - & - & 1 & 1 & 2 & {$[0,8 \%]$} \\
\hline 120 bis 150 & - & - & - & 3 & 3 & {$[1,3 \%]$} \\
\hline 200 & - & - & - & 2 & 2 & {$[0,8 \%]$} \\
\hline 300 & - & - & - & 1 & 1 & {$[0,4 \%]$} \\
\hline 500 & - & - & - & 1 & 1 & {$[0,4 \%]$} \\
\hline 1.000 & - & - & - & 1 & 1 & {$[0,4 \%]$} \\
\hline 16.500 & - & - & - & 1 & 1 & {$[0,4 \%]$} \\
\hline Gesamt & 20 & 76 & 63 & 78 & 237 & {$[100 \%]$} \\
\hline
\end{tabular}

11 bis 20 Freiwillige sind v. a. in mitgliederstärkeren MSO vertreten, und zwar mehrheitlich bei den MSO mit über 100 sowie mit 51 bis 100 Mitgliedern. Zudem verfügen die in der Analysestichprobe am häufigsten vertretenen Ü-100Mitglieder-Organisationen mehrheitlich auch über 41 bis 50 Engagierte.

Im Gesamtbild zeigt sich, dass die mitgliederreichsten MSO regelmäßig auch die höchsten Engagiertenzahlen aufweisen, die in einzelnen Organisationen mit über 100 Mitgliedern besonders hoch ausfallen können; andererseits ist in letzteren aber ggf. auch nur eine geringere Anzahl von 1 bis 10 Engagierten vorzufinden (Tab. 7.14). Umgekehrt sind auch in mitgliederärmeren Organisationen (11 bis 50) vereinzelt viele Freiwillige vorhanden, sodass Mitglieder- und Engagiertenzahlen fast deckungsgleich ausfallen.

Für die Testung, ob ein Zusammenhang zwischen den Mitglieder- und den Engagiertenzahlen besteht, wurde der Rangkorrelationskoeffizient nach Spearman berechnet. Es zeigt sich eine signifikante, starke positive Korrelation $\left(r h o=0,58^{* * *}\right)$ : Je höher die Mitgliederzahlen in einer MSO ausfallen, desto mehr Engagierte sind dort vorzufinden.

$\mathrm{Zu}$ erwähnen ist an dieser Stelle die Tatsache, dass sechs Funktionsträger angaben, dass ihre Organisationen weder über Hauptamtliche noch Engagierte verfügen, die bestimmte Aufgaben übernehmen. Das ruft die Assoziation eines wenig organisierten „Laissez faire, laissez aller“-Prinzips unter den 10 bis 50 und (in einer MSO) mehr als 100 Mitgliedern hervor, da die MSO in einer 
Marge von 2 bis 12 Tätigkeitsbereichen aktiv sind. Es könnte jedoch auch sein, dass der in der Frage verwendete Begriff der „Aufgaben“25 mit verbindlichen Ämtern (,Ehrenämtern“) und klar definierten Verpflichtungen gleichgesetzt wurde, deren Existenz in den MSO seitens der Funktionsträger (für Mitglieder) so nicht ausgemacht wurde.

\section{Entwicklung der Zahlen von Mitgliedern, Hauptamtlichen und Engagierten}

Signifikanter Anstieg von Hauptamtlichen in säkularen MSO

Die Beantwortung der Frage nach der Entwicklung der Zahlen der Mitglieder, Hauptamtlichen und Engagierten lässt für alle drei Statusgruppen in erster Linie sowohl eine Stabilität als auch eine Zunahme feststellen. ${ }^{26}$ Der Anstieg fällt für die Mitglieder (47 \%; $n=245)$ am größten und größer aus als die Stabilität (36 \%). Dieses gilt insbesondere für die religiösen Organisationen (RMO: $58 \%$ vs. MO: $41 \%$ ). Jedoch liegt auch für den Mitgliederschwund für alle MSO mit insgesamt 17 Prozent im Vergleich zu den beiden Gruppen der Engagierten und Hauptamtlichen der höchste Prozentwert vor.

Bei den hauptamtlich Beschäftigten dominiert eine Zunahme (51\%; $\mathrm{n}=$ 81) die Beständigkeit der Zahlen (44\%). Allerdings gilt der Anstieg insbesondere für säkulare Organisationen (65\% vs. $24 \%$ ), während in religiösen MSO die Stabilität hervorsticht (76\% vs. $27 \%$ ) und in diesen in Bezug auf die Hauptamtlichen auch kein einziger Rückgang vermerkt wird. Der $U$-Test zeigt, dass die Erhöhung der Anzahl von Hauptamtlichen insbesondere für die säkularen Vereinigungen zutrifft. ${ }^{27}$ Dies verweist auf deren professionelle Ausrichtungen und einen Professionalisierungstrend (vgl. auch Halm et al. 2020: 119; Friedrichs et al. 2020: 45).

Für die freiwillig Engagierten ist mit 52 Prozent insgesamt v. a. eine Stabilität festzustellen. Ein Anstieg von 34 Prozent steht in dieser Gruppe einem Rückgang von 14 Prozent gegenüber, der mit dem Mitgliederschwund zu korrespondieren scheint. Eine differenzierte Betrachtung zeigt, dass die Zunahme von Freiwilligen insbesondere für die religiösen MSO zutrifft (42\% vs. $30 \%$ ), allerdings nicht in einem statistisch signifikanten Ausmaß.

25 „Gibt es in Ihrer Organisation Personen, die freiwillig und unbezahlt bestimmte Aufgaben übernehmen?"

${ }^{26}$ Für die Ergebnispräsentation wurden die vier Werte „stark gesunken“ und ,gesunken“ sowie „gestiegen“ und „stark gestiegen“ der sechsstufigen Antwortskala zusammengefasst (zwei weitere Skalenpunkte waren: ,nicht verändert“, „haben wir nicht").

${ }^{27} \underline{U}$-Test: $U=455 ; z=-3,15 ; p=0,002 ; r=0,35 ; \mathrm{Md}_{\mathrm{S}}=4 ; \mathrm{Md}_{\mathrm{R}}=3$. 
In Bezug auf die Verfügbarkeit von personellen Ressourcen ist herauszustellen, dass auch die Studie des SVR-Forschungsbereichs für die MSO einen seit einigen Jahren zu registrierenden „Professionalisierungsschub“ (Friedrichs et al. 2020: 45; I. 2.1) konstatiert. Die MSO sind demnach nicht generell als strukturschwach einzustufen, allerdings bleibt die Disponibilität von Engagierten und Ehrenamtlichen von anhaltend großer Bedeutung (vgl. auch Halm et al. 2020: 119; I. 2.2).

\subsubsection{Die freiwillig Engagierten: Personengruppen, Mobilisierung, eingeschätzte Motivstruktur}

Personengruppen: Bei ähnlichen Anteilen 2 Statusgruppen am häufigsten vorhanden

Wie auch für die Mitglieder wurden die Funktionsträger der MSO gebeten, die in ihrer Organisation engagierten Personen mit einer sechsstufigen Antwortskala ${ }^{28}$ den 5 Personenkreisen „Berufstätige/Selbstständige“, „Schüler und Studierende“, „Hausfrauen/Hausmänner“, „Arbeitslose/Arbeitssuchende“ und „Rentner“ zuzuordnen.

Eine grobe Einteilung fördert zunächst zutage, dass in rund einem Viertel (25\%) und damit in der Mehrzahl der MSO zwei Personenkreise, in jeweils circa 20 bis 21 Prozent der Vereinigungen drei, fünf oder eine und in 15 Prozent vier der Personengruppen anzutreffen sind (Tab. 7.15).

Die Kombinationen der verschiedenen Personenkreise unter den Engagierten sind - im Unterschied zur Verteilung unter den Mitgliedern - in allen MSO also zu recht ähnlichen Anteilen verbreitet (Tab. 7.15).

Eine genauere Betrachtung nach innerhalb dieser Blöcke wiederum mehrheitlich vorhandenen Statusgruppen zeigt v. a. die ausschließliche Präsenz von Berufstätigen in 15 Prozent der MSO, darüber hinaus aber auch die Dominanz von Berufstätigen in den Zweier-, den Dreier- und den Fünfer-Gruppen (Tab. 7.15). In letzteren sind aber mit 8 Prozent häufiger auch alle Personenkreise zu gleichen Anteilen vorhanden (in Tab. 7.15 nicht gesondert ausgewiesen).

\footnotetext{
${ }^{28}$ Sechsstufige Antwortskala: ,,alle“, „mehr als die Hälfte“, ,ungefähr die Hälfte“, ,,weniger als die Hälfte“, ,,keine“, ,,weiß nicht“.
} 
Tab. 7.15 Engagierte nach Personengruppen (im Vergleich zu Mitgliedern)

\begin{tabular}{lrrr}
\hline Gruppen & Häufigkeit & Prozent & $\begin{array}{c}\text { Vergleich } \\
\text { Mitglieder (in \%) }\end{array}$ \\
\hline 1 Gruppe & & & \\
\hline 1 Gruppe: Berufstätige & 8 & 3,4 & 7,6 \\
\hline 1 Gruppe: Rentner & 5 & 2,1 & 1,6 \\
\hline 1 Gruppe (sonstige) & 49 & 20,8 & 9,6 \\
\hline Gesamt & & & \\
\hline 2 Gruppen & 16 & 6,8 & 5,2 \\
\hline 2 Gruppen: Berufstätige, Rentner & 15 & 6,4 & 4,4 \\
\hline 2 Gruppen: Berufstätige, SuS & 13 & 5,5 & 1,6 \\
\hline 2 Gruppen: Berufstätige, Hausfrauen & 14 & 5,9 & 3,5 \\
\hline 2 Gruppen (sonstige) & 58 & 24,6 & 14,7 \\
\hline Gesamt & & & \\
\hline 3 Gruppen & 11 & 4,7 & 4,4 \\
\hline 3 Gruppen: Berufstätige, SuS, Hausfrauen & 10 & 4,2 & 4,8 \\
\hline 3 Gruppen: Berufstätige, Hausfrauen, Rentner & 8 & 3,4 & 4,8 \\
\hline 3 Gruppen: Berufstätige, SuS, Rentner & 18 & 7,6 & 5,5 \\
\hline 3 Gruppen (sonstige) & 47 & 19,9 & 19,5 \\
\hline Gesamt & & & \\
\hline 4 Gruppen & 14 & 5,9 & 9,6 \\
\hline 4 Gruppen (o. Arbeitslose) & 11 & 4,7 & 4,3 \\
\hline 4 Gruppen (sonstige) & 10 & 4,2 & 6,4 \\
\hline 4 Gruppen (o. Rentner) & 35 & 14,8 & 20,3 \\
\hline Gesamt & 47 & 19,9 & 35,9 \\
\hline 5 Gruppen & 236 & 100,0 & \\
\hline Gesamt & & \\
\hline & & & \\
\hline
\end{tabular}

Das im Vergleich zu den Mitgliedern (7.1.2.1) unter den Engagierten insgesamt ausbalanciertere Verhältnis der Gruppen verdeutlicht die Varianz von enger (Einer-, Zweier-Gruppen), aber auch breiter gefassten Kreisen von Freiwilligen. Menschen unterschiedlicher Generationen und Erwerbsstatus sind gemeinsam aktiv. Für Arbeitslose könnte die Übernahme bestimmter Aufgaben eine tagesstrukturierende und sinnstiftende Funktion übernehmen, die ggf. mit der Intention einhergeht, wieder in Arbeit zu kommen.

Wie auch bei den Mitgliedern wäre an dieser Stelle eine Vergleichsziehung zu den in den Selbstorganisationen von Autochthonen vorhandenen Engagiertenstrukturen interessant, die allerdings mangels empirischer Befunde ebenfalls nicht möglich ist. 


\section{Mobilisierung der Engagierten}

Religiöse MSO rekrutieren signifikant mehr Ehrenamtliche

Bei der Einschätzung der Aussagen zu den freiwillig Engagierten ${ }^{29}$ wird deutlich, dass sich mehrheitlich genügend Freiwillige gewinnen (61\%) und sich ehrenamtliche Leitungspositionen mit 51 Prozent gut besetzen lassen. Bei den anderen 39 bzw. 49 Prozent ist diesbezüglich von Schwierigkeiten oder Unzufriedenheit auszugehen. ${ }^{30}$

Ein Vergleich der Prozentwerte zwischen säkularen und religiösen MSO zeigt, dass letztere in einem höheren Ausmaß genügend Freiwillige (70\% vs. $58 \%$ ) sowie ausreichend Personen für die Ehrenämter aktivieren können (69 \% vs. $44 \%$ ) (Differenz: 12 und 15 Prozentpunkte). Anhand statistischer Berechnungen kann jedoch lediglich in Bezug auf die Besetzung von ehrenamtlichen Leitungspositionen für die religiösen Vereinigungen eine signifikant größere Bedeutung ausgemacht werden. ${ }^{31}$

Angesichts der statistischen Testergebnisse kann die Hypothese

H4 $_{1}$ : Religiöse MSO sind hinsichtlich der Gewinnung von Freiwilligen erfolgreicher als nicht-religiöse

nicht hinlänglich, sondern nur in Bezug auf die Gewinnung von Personen für Ehrenämter ${ }^{32}$ bestätigt werden. Sie ist an dieser Stelle aber auch mit anderen, bereits dargelegten Befunden zu den Engagierten ins Verhältnis zu setzen. So ist hinsichtlich der Anzahl der Engagierten eine signifikant größere Anzahl in den religiösen MSO auszumachen und ebenfalls ist insbesondere für religiöse Vereinigungen eine Zunahme der Anzahl von Freiwilligen zu konstatieren; diese fällt im Vergleich mit der Gruppe der säkularen MSO jedoch nicht signifikant aus. In der Summe kann dennoch davon ausgegangen werden, dass die religiösen MSO in dem Bestreben, mehr Freiwillige zu gewinnen, positivere Resultate erzielen. Die Ergebnisse zeigen aber auch, dass das Ehrenamt sowie die Engagierten die Stützpfeiler der Tätigkeiten und

29 „Wir können genügend Freiwillige gewinnen“; „Für freiwillig und unbezahlt ausgeführte Leitungspositionen und Ämter (,Ehrenämter") finden wir genug Freiwillige“; „Die Freiwilligen sind vorwiegend an zeitlich befristeten Tätigkeiten interessiert“.

${ }^{30}$ Die Ergebnispräsentation basiert auf einer Zusammenfassung der vierstufigen Antwortskala zu den zwei Kategorien „,Trifft voll und ganz zu/Trifft eher zu“ und ,Trifft eher nicht zu/Trifft überhaupt nicht zu“".

${ }^{31} U$-Test: $U=3614 ; z=-3,43 ; p=0,001 ; r=0,23 ; \mathrm{Md}_{\mathrm{S}}=3 ; \mathrm{Md}_{\mathrm{R}}=2$.

${ }^{32}$ Siehe für eine Definition des Begriffs ,Ehrenamt“ II. 3.1, Fußnote 2. 
Angebote in den religiösen MSO sind und damit eine Abhängigkeit von ihrem Einsatz besteht. Gerade aufgrund ihrer fundamentalen Bedeutung ist die Rekrutierung essenziell und aufgrund des Bedarfs scheinbar noch zu verstärken.

Dass die Freiwilligen überwiegend an zeitlich befristeten Tätigkeiten interessiert sind, wird von 68 Prozent der MSO bejaht und dies sowohl von säkularen als auch religiösen MSO gleichermaßen mehrheitlich (MO: $72 \%$; RMO: $60 \%$ ). Damit scheinen die Einstufungen zu dieser Aussage den in der Forschung ausgemachten Trend des unter Engagierten vorherrschenden Interesses an einer unverbindlichen Übernahme kurzfristiger Tätigkeiten zu bestätigen (vgl. Friedrichs et al. 2020: 21). Auch der allgemeine Forschungsbefund zu hinsichtlich der Gewinnung von Ehrenamtlichen bestehenden Schwierigkeiten bzw. eines bei der Besetzung von Ehrenämtern zu registrierenden Nachwuchsproblems (BMFSFJ 2017: 136 ff.) erweist sich im Rahmen der Studie in NRW teilweise und insbesondere für die nicht-religiösen MSO als zutreffend; bei den religiösen Vereinigungen ist hingegen eine bessere Ehrenamtsrekrutierungslage festzustellen.

In einer nächsten Frage war in einer Itembatterie zu beantworten, auf welche Art und Weise Freiwillige für die Übernahme unbezahlter Aufgaben erreicht und gewonnen werden. Es zeigt sich, dass Freiwillige in den MSO mit 93 Prozent insbesondere durch die Ansprache von Freunden und Bekannten, durch eine oder mehrere motivierende und begeisternde Person(en) in der Organisation (jeweils $85 \%$ ) wie auch mit regelmäßigen Veranstaltungen $(79 \%)$ mobilisiert werden können. Eine in den Familien gelebte Tradierung des Mitmachens wird für 69 Prozent der MSO geltend gemacht. 66 Prozent der Funktionsträger geben an, dass Eltern durch die Teilnahme von Kindern motiviert werden können. Der Öffentlichkeitsarbeit kommt mit 58 Prozent in den MSO für die Gewinnung von Engagierten eine größere Bedeutung zu als angebotenen Gegenleistungen (35\%), wie etwa Aufwandsentschädigungen oder Weiterbildungen, sowie der Ansprache von unbekannten Personen durch Mitglieder und Mitarbeiter (22\%).

\section{- Statistische Tests}

Auf Basis einer Explorativen Faktorenanalyse (EFA) lassen sich drei Faktoren identifizieren, denen sich die genannten Mobilisierungsmaßnahmen jeweils zuordnen lassen (Tab. 7.16) und die wie folgt benannt werden können:

1) Öffentlichkeitsarbeit - das v. a. auch von Einzelpersonen abhängige Bemühen, die Attraktivität von Leistungen bzw. Angeboten zu unterstreichen, um Personen für die Mitwirkung in der Organisation zu gewinnen bzw. zu begeistern; 
2) Nutzbarmachung von organisationsintern vorhandenen (familiären) Beziehungsstrukturen und persönlichen Netzwerken;

3) Akquise von externen, nicht-bekannten Personen durch Gegenleistungen.

Tab. 7.16 Rotierte Faktorladungsmatrix zu Mobilisierungsmaßnahmen

\begin{tabular}{|c|c|c|c|}
\hline & \multicolumn{3}{|c|}{ Faktoren } \\
\hline & 1 & 2 & 3 \\
\hline $\begin{array}{l}\text { Durch eine oder mehrere motivierende und begeisternde } \\
\text { Person(en) in unserer Organisation }\end{array}$ & ,818 & ,022 & ,058 \\
\hline Mit einer erfolgreichen Öffentlichkeitsarbeit & ,727 & 195 & ,294 \\
\hline Mit regelmäßigen Veranstaltungen als gute Gelegenheiten & ,711 & ,204 & 091 \\
\hline $\begin{array}{l}\text { Durch „Familientraditionen“: Familienmitglieder machen auch mit } \\
\text { bzw. haben früher mitgemacht }\end{array}$ &,- 071 & ,835 & 077 \\
\hline $\begin{array}{l}\text { Durch die Motivation von Eltern, deren Kinder an unseren Aktivitä- } \\
\text { ten teilnehmen }\end{array}$ &, 229 &, 778 & ,033 \\
\hline Indem Mitglieder/Mitarbeiter Freunde und Bekannte ansprechen & ,248 & ,583 & 032 \\
\hline $\begin{array}{l}\text { Mit Gegenleistungen (z. B. Aufwandsentschädigung, Weiterbildung, } \\
\text { unseren Angeboten) }\end{array}$ & ,137 & ,008 & ,838 \\
\hline Indem Mitglieder/Mitarbeiter unbekannte Personen ansprechen & 138 & 098 &, 819 \\
\hline
\end{tabular}

Extraktionsmethode: Hauptkomponentenanalyse. Fehlende Werte: paarweiser Fallausschluss. Rotationsmethode: Varimax mit Kaiser-Normalisierung. Maß der Stichprobeneignung nach Kaiser-Meyer-Olkin (KMO-Test): 0,685. Eigenwerte $>1,0 ;$ Faktorladung $\geq+/-0,5 ; \mathrm{n}=197-225$.

Engagiertenmobilisierung: Relevanz von Gegenleistung (MO) versus Motivierung von Eltern (RMO)

Die Motivation von Eltern und auch regelmäßige Veranstaltungen besitzen mit einer Differenz von 18 bzw. 11 Prozentpunkten für die religiösen Vereinigungen hinsichtlich der Rekrutierung von Freiwilligen eine größere Bedeutung. Dieses zeigt sich in geringfügigerem Ausmaß für die Familientradition (+7 Prozentpunkte); in den säkularen MSO ist hingegen die Ansprache von Freunden und Bekannten sowie die Öffentlichkeitsarbeit etwas wichtiger $\left(+7\right.$ und +6 Prozentpunkte).$^{33}$ Der deutlichste Unterschied zeigt sich bei der Einschätzung der Relevanz von Gegenleistungen, die

${ }^{33}$ Motivation von Eltern (RMO: $78 \%$ vs. MO: $60 \%$ ) I regelmäßige Veranstaltungen (RMO: $86 \%$ vs. MO: $75 \%$ ) | Familientradition (RMO: $74 \%$ vs. MO: $67 \%$ ) I Ansprache von Freunden und Bekannten (RMO: 88 \% vs. MO: $95 \%$ ) I Öffentlichkeitsarbeit (RMO: $53 \%$ vs. MO: $59 \%)$. 
in 18 Prozent der religiösen MSO und in 44 Prozent der nicht-religiösen bedeutsam sind (Differenz: 26 Prozentpunkte).

Anhand der Berechnung des $U$-Tests kann für die Mobilisierungsoption der Vorhaltung von Gegenleistungen für die säkularen Vereinigungen sowie für den Aspekt der Motivierung von Eltern hingegen für die religiösen Gemeinden jeweils eine signifikant größere Relevanz ermittelt werden. ${ }^{34}$

\section{- Hypothesenprüfung und Ergebnisdiskussion}

Die statistischen Tests zeigen, dass der zweite Teil der Hypothese Nummer 4 im Rahmen der Befragung nicht mit statistischen Signifikanzen bestätigt werden kann:

$\mathbf{H 4}_{2}$ : Familientraditionen, regelmäßige Veranstaltungen sowie die persönliche Ansprache von Freunden/Bekannten im religiösen Feld sind besonders wirksame Faktoren für die Rekrutierung von Engagierten in religiösen MSO.

Im anreiztheoretischen Abschnitt (II. 4.4.2) wurde dargelegt, dass ein potenzieller Rekrutierungsvorteil von religiösen Organisationen mehrfachbedingt sein kann. Neben einer bewussten wertegeleiteten, religiös verankerten Entscheidung (Selbstselektion) für eine Beteiligung kann eine Person über die Familie in eine religiöse Organisation hineinsozialisiert worden sein. In diesem Fall sollten dann in Bezug auf das freiwillige Engagement auch Familientraditionen relevant sein, sodass die aktive Mitwirkung potenziell auch unhinterfragt stattfinden könnte, da es sich um eine generationenübergreifende Konvention handelt. In religiösen Gemeinden, als viel und regelmäßig besuchte Orte, können Mitglieder und Engagierte zudem über persönliche Beziehungen und direkte Ansprachen im Rahmen von religiösen Veranstaltungen gewonnen werden (u. a. Cnaan/Curtis 2013: 23; Wilson/Janoski 1995: 138). Erwartungshaltungen und Aufforderungen von anderen Gruppenmitgliedern oder religiösen Amtsträgern, sich einzubringen, werden nicht so einfach abzulehnen sein (II. 4.4.2). Zudem kann bei einer häufigen Inanspruchnahme der Angebote ein Gefühl der persönlichen Verpflichtung bestehen, sich durch eigene unbezahlte Leistungserbringung gegenüber der Gemeinschaft revanchieren zu müssen (Klöckner 2016: 155, 432).

${ }^{34}$ Mit Gegenleistungen $U$-Test: $U=2997 ; z=-3,68 ; p=0,000 ; r=0,26 ; \mathrm{Md}_{\mathrm{S}}=3$;

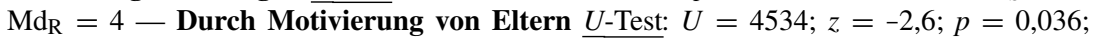
$r=0,15 ; \operatorname{Mds}_{\mathrm{S}}=2 ; \operatorname{Md}_{\mathrm{R}}=2$. 
Wenngleich sich im Rahmen der in NRW erfolgten Befragung eine größere Bedeutung von Familientraditionen und regelmäßigen Veranstaltungen für religiöse MSO abzeichnet, wird die Relevanz von bestehenden Beziehungsstrukturen lediglich für den Aspekt der Motivierung von Eltern, deren Kinder in den religiösen Vereinigungen an Aktivitäten teilnehmen, mit statistischer Signifikanz bestätigt. Dies ist ein Hinweis darauf, dass Kinder bzw. ihre Eltern und damit Familienstrukturen innerhalb der befragten religiösen MSO von größerer Bedeutung sind, da das Angebot kinderfreundlich ist und durch das Vorhandensein von Kindern und deren Eingebundensein in der religiösen Vereinigung günstige Anlässe für die Aufnahme eines Engagements oder Ehrenamts durch die direkte Ansprache von Eltern gegeben ist (II. 4.3.3.1). Diesbezüglich von Belang ist hier auch die im anreiztheoretischen Kapitel (II. 4.4.2) thematisierte Multifunktionalität von religiösen MSO und insbesondere Moscheevereinen als viele Bedürfnisse befriedigende Dienstleistungszentren für (Groß-)Familien, zu denen in Deutschland keine alternativen Einrichtungen mit einer vergleichbaren Angebotsvielfalt existieren (Ceylan 2006: 123 ff.; Ceylan 2008; vgl. auch Sauer 2011: 223; Halm/Sauer 2005: 81). Sie übernehmen die religiöse Grundversorgung von Mitgliedern und deren Angehörigen sowie die Vermittlung religiöser Traditionen. Zudem können die Herkunftskultur und Muttersprache gepflegt und gruppenspezifische Interessen diskutiert werden (Handy/Greenspan 2009; Carabain/Bekkers 2010). Zahlreiche formelle Veranstaltungen, aber auch informelle Zusammenkünfte auf dem Moscheegelände machen die Moscheegemeinden zu lebendigen Aufenthaltsorten, die sowohl religiöse als auch nicht-religiöse Aktivitäten anbieten, aber daneben auch schlicht Orte der Begegnung und des Beisammenseins sind, die die ganze Familie involvieren. Kinder werden beispielsweise durch die Möglichkeit des spontanen Fußballspielens oder anderer Freizeitaktivitäten angezogen. Das Eingebundensein von Kindern und deren häufige Aufenthalte in der Moschee könnten die Motivation der Eltern auslösen oder verstärken, sich aktiv in die Gemeinde einzubringen.

Der für nicht-religiöse Organisationen wichtige nutzenorientierte Aspekt des Erhalts von in Geld umrechenbaren Gegenleistungen (z. B. Aufwandsentschädigungen, Weiterbildungen) könnte mit der professionellen Ausrichtung der MSO und auch ex negativo erklärt werden. In religiösen Organisationen sind neben intakten Familienstrukturen insbesondere in religiösen Überzeugungen verankerte immaterielle Anreize von Belang, wie dem Wunsch gute Taten zu vollbringen und wertekonform und damit auch gottgefällig zu handeln (Harris 2003; Smidt 2003c; II. 4.4.2). 
Die anderen sechs zur Disposition gestellten Mobilisierungsaspekte (Items) ${ }^{35}$ scheinen hingegen mit geringfügigen Varianzen sowohl für die religiösen als auch säkularen Vereinigungen bedeutsam zu sein. Insofern erweist sich die Annahme eines spezifischen Rekrutierungsprofils der religiösen MSO als theoretisch überspannt und hinsichtlich der beiden fokussierten Organisationstypen als unverhältnismäßig. Allerdings sollte dies anhand einer größeren Analysestichprobe insbesondere auch mit Blick auf die in der Hypothese für religiöse MSO geltend gemachte größere Bedeutung von Familientraditionen und regelmäßigen Veranstaltungen als gute Gelegenheiten für die Gewinnung von Freiwilligen nochmals überprüft werden.

\section{Einschätzung der Motive, von denen sich die Engagierten am stärksten leiten lassen}

Die Motive der Engagierten anhand subjektiver Einschätzungen der Funktionsträger zu erheben, beruht auf der theoretischen Prämisse, dass die Motive der Individuen auf die Gruppenebene ausgerichtet sind und diese damit unterstützen. Die MSO bieten die lebensweltlichen Opportunitäts- und Gelegenheitsstrukturen, in denen die individuellen Engagements eingebettet sind und in denen sich subjektive dispositionelle Eigenschaften realisieren können. Insofern sollten individuelle Motive und Organisationsziele miteinander korrespondieren. Es ist somit von einer Interdependenz bzw. Verquickung zwischen der Mikro- und der Mesoebene und sich wechselseitig verstärkenden Phänomenen auszugehen (II. 4.3.3.2).

Da nicht die Engagierten selbst befragt, sondern die Einschätzungen der Organisationsvertreter in den Blick genommen wurden, wurden nicht die tatsächlichen Beweggründe der Engagierten auf der Individualebene erhoben. ${ }^{36}$ Anzunehmen ist, dass die Befragten Ziele und Interessen ihrer Organisation oder möglicherweise auch persönliche Motive kommuniziert haben. Zudem ist zu vermuten, dass seitens der Funktionsträger ein „Matching“ vorgenommen wurde, d. h. eine Passung oder Abgleichung von den Zielen und Interessen der MSO sowie den dort offerierten Realisierungsmöglichkeiten einerseits mit den eingeschätzten

\footnotetext{
35 Durch Ansprache von Freunden und Bekannten; durch Ansprache von unbekannten Personen; durch motivierende und begeisternde Person(en); mit regelmäßigen Veranstaltungen; durch eine in den Familien gelebte Tradierung des Mitmachens; mit einer erfolgreichen Öffentlichkeitsarbeit.

${ }^{36}$ Für subjektive Beweggründe und die Entscheidung zu einem Engagement zentrale extraorganisationale Situationsumstände, die u. a. die individuelle Lebenssituation, sozioökonomische Merkmale und verfügbare Ressourcen umfassen, sind selbstverständlich immer hochrelevant (II. 4.3.3.1 und II. 4.3.3.2). Sie bleiben im Kontext dieser Erhebungsart auf der Mesoebene jedoch unbeachtet.
} 
stärksten Motiven der Engagierten andererseits. Die Motivabfrage besitzt damit quasi eine Dummy-Funktion, eine testende Stellvertreterfunktion, die im Vergleich mit anderen Ergebnissen der Befragung - Expertengesprächen ähnelnd - gleichwohl interessante Auskünfte gibt, da die menschlichen Motive im Kontext organisationaler Rahmenbedingungen betrachtet werden.

Es zeigt sich, dass die Funktionsträger von einer Vielfalt der Motive ausgehen (Abb. 7.6). Ganz oben rangieren mit jeweils über 93 Prozent die Einschätzungen, dass die Engagierten Freude an der Tätigkeit haben (94\%) und etwas für andere tun bzw. anderen helfen wollen $(93 \%){ }^{37}$

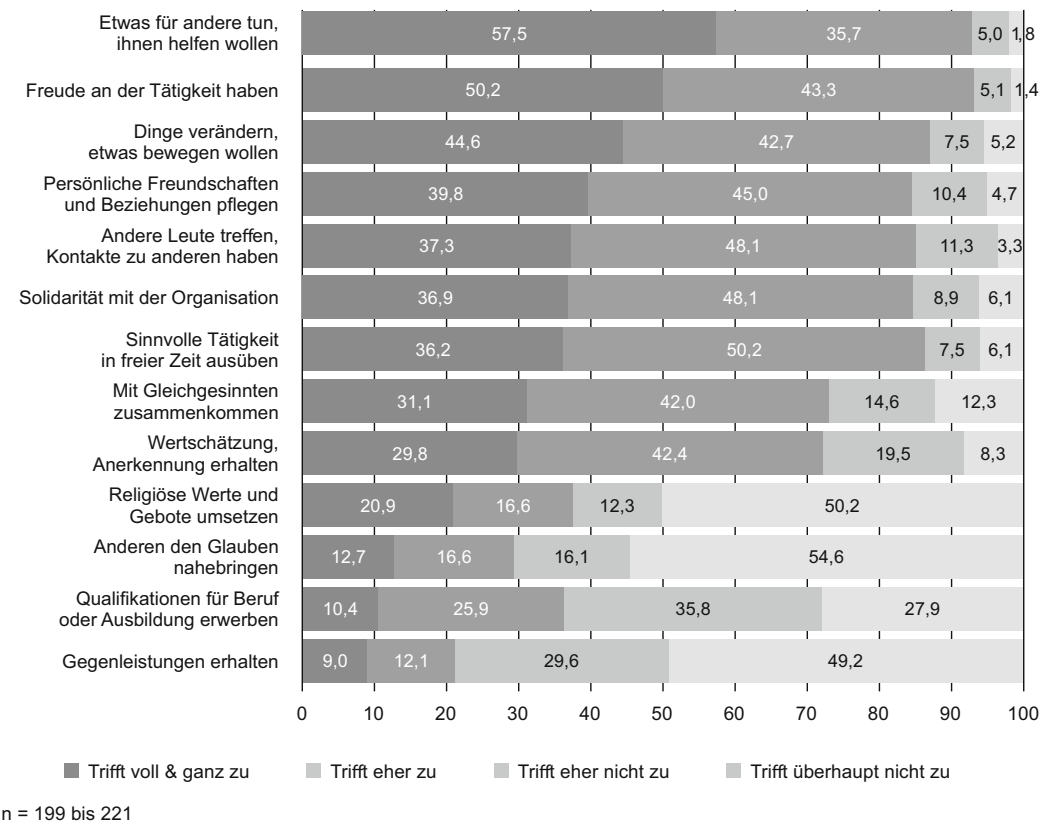

Abb. 7.6 Einschätzung der Motive (in \%)

${ }^{37}$ Die Ergebnispräsentationen im Fließtext basieren im Folgenden häufiger auf einer Zusammenfassung der vierstufigen Antwortskala zu den zwei Kategorien , Trifft voll \& ganz zu/Trifft eher zu“ und „Trifft eher nicht zu/Trifft überhaupt nicht zu“. In den Balkendiagrammen werden die Prozentwerte hingegen jeweils detailliert für alle vier Skalenausprägungen und nach dem Wert einer kompletten Zustimmung in absteigender Sortierung dargestellt (6.2). 
Für jeweils über 85 Prozent der in den MSO Engagierten wird angegeben, dass sie Dinge verändern bzw. etwas bewegen wollen ( $87 \%$ ), in ihrer Freizeit eine sinnvolle Tätigkeit ausüben ( $86 \%$ ) oder andere Leute treffen und Kontakte zu anderen haben möchten (85\%). Dem entsprechend wird die Pflege persönlicher Freundschaften und Beziehungen gleichauf als ein bedeutendes Movens ( $85 \%$ ) eingestuft. Als ebenso relevant wie die Beziehungspflege wird für die in allen MSO Engagierten eine sich auf ihr Eintreten für und die Solidarität mit der Organisation gründende Unterstützung (85\%) ausgemacht.

Das darin implizierte Zusammengehörigkeits- oder Gemeinschaftsgefühl aufgrund geteilter Ziele oder Anschauungen bestätigt sich durch die Einschätzung, sich in der MSO zu engagieren, um mit Gleichgesinnten zusammenzukommen (73\%). Dies wird für die in religiösen MSO freiwillig Tätigen als bedeutsamer eingeschätzt (RMO: $82 \%$ vs. MO: $69 \%$ ). Für letztere wird insbesondere auch die Möglichkeit, religiöse Werte und Gebote umzusetzen ( $86 \%$ vs. $13 \%$; alle MSO: $38 \%$ ) und - in geringerem Ausmaß - anderen den Glauben nahebringen zu wollen, als relevant eingestuft (65\% vs. $12 \%$; alle MSO: $29 \%$ ). Hinsichtlich dieser beiden religiösen Motive besteht zwischen den beiden Organisationstypen damit eine Differenz von 73 bzw. 53 Prozentpunkten, wodurch sich das Motivprofil der Engagierten in religiösen und säkularen MSO deutlich voneinander abgrenzen lässt.

Das Bedürfnis der Engagierten, Wertschätzung und Anerkennung zu erhalten, wird für fast drei Viertel der MSO als bedeutsam befunden (72\%). Die religiösen und nicht-religiösen MSO unterscheiden sich darin kaum. Allerdings wird in säkularen Vereinigungen für die Freiwilligen deutlich häufiger der Wunsch ausgemacht, über ihre Engagements Qualifikationen für Beruf oder Ausbildung zu erwerben (MO: $41 \%$; RMO: $26 \%$; alle MSO: $36 \%$ ) sowie Gegenleistungen wie etwa Aufwandsentschädigungen zu erhalten oder Angebote der Organisation nutzen zu können (MO: $27 \%$; RMO: $8 \%$; alle MSO: $21 \%$ ). Insgesamt wird den beiden letztgenannten Motiven im Vergleich zu den vorgenannten jedoch für alle MSO eine deutlich geringere Relevanz beigemessen (Abb. 7.6).

Angesichts der obigen Befunde lässt sich resümieren, dass neben lust- und geselligkeitsorientierten Beweggründen vorrangig Hilfeleistungen für andere und das Interesse an Weiterentwicklung und Optimierung vorgefundener Gegebenheiten als relevante Triebfedern ausgemacht werden. Dieses entspricht Ergebnissen von auf der Individualebene durchgeführten Befragungen von türkeistämmigen Personen, die ein Mischungsverhältnis von Nutzenorientierung, Altruismus und Hedonismus feststellen (Halm/Sauer 2005: 118 f.; Sauer 2011: 231 f.; s. auch Klie 2018: 444 f.). 
Zugleich ähneln die Befunde aber auch den für die deutsche Gesamtbevölkerung identifizierten Motivlagen. Wie auch die MSO-Befragung weisen der personenbezogene Freiwilligensurvey 2014 (FWS) und eine weitere bevölkerungsrepräsentative Befragung des Allensbacher Instituts nach, dass für 94 bzw. 95 Prozent der Engagierten Spaß und für jeweils 82 Prozent das Zusammenkommen mit anderen Menschen, darüber hinaus aber auch der Wunsch, etwas zu bewegen (IfD: $83 \%$ ) oder die Gesellschaft mitzugestalten (FWS: $81 \%$ ), oben rangierende Beweggründe darstellen (Müller et al. 2016: 418 f.; IfD Allensbach 2013: 27 ff.). Diskrepanzen zeigen sich hinsichtlich des Aspekts des Erhalts von Anerkennung und Wertschätzung, der seitens der MSO-Vertreter auf einem (deutlich) höheren Niveau als bedeutsam eingestuft wird (FWS: -40, IfD: -18 Prozentpunkte), wohingegen die Erwartung des Qualifikationserwerbs (für Beruf und Ausbildung) im Vergleich zu den Befunden des Freiwilligensurveys als für die in den MSO Engagierten weniger relevant gewichtet wird ( -16 Prozentpunkte), jedoch wiederum höher ausfällt als in der Allensbacher Studie (+17 Prozentpunkte). ${ }^{38}$

Während die Korrespondenzen einerseits zur Annahme verleiten, dass die Funktionsträger mit ihren Einschätzungen ganz richtig liegen, verweisen die Abweichungen andererseits auf die unterschiedlichen Erhebungsarten: Im Organisationskontext wird der Befriedigung individueller Geltungsbedürfnisse durch gruppenbezogene Wertschätzung seitens der Funktionsträger mehr Gewicht als dem persönlichen Eigennutzen der Engagierten eingeräumt.

\section{- Statistische Tests}

Anhand einer Explorativen Faktorenanalyse (EFA) lassen sich die fünf im Folgenden beschriebenen Gruppen (Faktoren) inhaltlich zusammengehöriger Motive identifizieren (Tab. 7.17):

${ }^{38}$ MSO: „Wertschätzung, Anerkennung erhalten“ (72 \%) I FWS: „Ansehen und Einfluss gewinnen“ (32 \%) I IfD Allensbach: ,weil ich Wertschätzung bzw. Anerkennung erhalte“ (54 \%) - MSO: „Qualifikationen für Beruf oder Ausbildung erwerben“ (36 \%) I FWS: „Qualifikationen erwerben“ (52 \%) I IfD Allensbach: „,weil es mir beruflich bzw. für die Ausbildung etwas bringt" (19\%).

An dieser Stelle ist einzuräumen, dass die Vergleiche zu Befunden (Ähnlichkeiten und Diskrepanzen) von Motivuntersuchungen von Engagierten auf der Individualebene gezogen und z. T. Items verglichen werden, die inhaltlich nicht deckungsgleich formuliert sind, und dass die Vergleiche somit hinken; z. B. „Ansehen und Einfluss gewinnen“ (FWS) — „Wertschätzung und Anerkennung erhalten“ (MSO/IfD Allensbach). 
Tab.7.17 Rotierte Faktorladungsmatrix zu Engagementmotiven

\begin{tabular}{|c|c|c|c|c|c|}
\hline & \multicolumn{5}{|c|}{ Faktoren } \\
\hline & 1 & 2 & 3 & 4 & 5 \\
\hline $\begin{array}{l}\text { Persönliche Freundschaften und Beziehungen } \\
\text { pflegen }\end{array}$ &, 833 & 034 &, 041 & 179 &, 129 \\
\hline Andere Leute treffen, Kontakte zu anderen haben & ,796 &,- 066 & ,175 & , 198 &,- 054 \\
\hline Freude an der Tätigkeit haben & ,674 & 181 &, 006 &,- 216 & 469 \\
\hline Sinnvolle Tätigkeit in freier Zeit ausüben &, 584 &,- 025 & ,127 & ,382 & 055 \\
\hline Religiöse Werte und Gebote umsetzen &,- 064 & ,928 &,- 132 & 111 & 036 \\
\hline Anderen den Glauben nahebringen & ,091 & ,909 & 069 & ,162 & 033 \\
\hline $\begin{array}{l}\text { Gegenleistung erhalten (Aufwandsentschädigung, } \\
\text { Angebote der Organisation) }\end{array}$ & ,087 &,- 122 & ,822 &,- 122 &,- 063 \\
\hline $\begin{array}{l}\text { Qualifikationen für Beruf oder Ausbildung } \\
\text { erwerben }\end{array}$ & ,106 & 081 & ,784 & ,084 & ,061 \\
\hline Wertschätzung, Anerkennung erhalten & , 123 &,- 039 &, 519 & ,335 &, 333 \\
\hline Mit Gleichgesinnten zusammenkommen & ,134 & ,216 &,- 105 & ,786 & 060 \\
\hline Solidarität mit der Organisation & ,312 & ,147 & 173 &, 685 &, 040 \\
\hline Etwas für andere tun, ihnen helfen wollen & , 139 &, 113 &,- 006 & ,034 & ,883 \\
\hline Dinge verändern, etwas bewegen wollen &, 014 &,- 234 & 378 & ,394 &, 534 \\
\hline
\end{tabular}

Extraktionsmethode: Hauptkomponentenanalyse. Fehlende Werte: paarweiser Fallausschluss. Rotationsmethode: Varimax mit Kaiser-Normalisierung. Maß der Stichprobeneignung nach Kaiser-Meyer-Olkin (KMO-Test): 0,706. Eigenwerte $>1,0 ;$ Faktorladung $\geq+/-0,5 ; \mathrm{n}=199-221$.

1) Engagement aufgrund des Bedürfnisses nach sozialem Anschluss und lustorientierter Selbstverwirklichung. Hier steht das Bedürfnis nach Affiliation im Vordergrund. Die Zugehörigkeit zu einer Gruppe und der soziale Austausch gehen mit einer sinngebenden und (lebens-)lustorientierten Selbstverwirklichung einher; subjektive Potenziale, Interessen und Neigungen können entfaltet und eingesetzt werden.

2) Engagement aufgrund religiöser Überzeugungen. Hier dominiert religionskonformes Verhalten aufgrund religiös verankerter, verinnerlichter Normen und Werte, von denen das Individuum überzeugt ist und die es in der Praxis umsetzen möchte.

3) Engagement aufgrund eines konkreten Eigennutzens und individueller Geltungsbedürfnisse.

Bedeutsam sind der Erhalt von (im-)materiellen Gratifikationen, das Erwirken von persönlichem Fortschritt und Weiterentwicklung durch Wissensaneignung sowie der Erhalt von Wertschätzung und Anerkennung durch andere. Bei letzterem geht es um Selbstbestätigung und die Erlangung eines positiven Selbstwertgefühls durch Fremdachtung. 
4) Engagement aufgrund des Gefühls von Zusammengehörigkeit und Gleichgesinntheit in der Organisation. Hier stehen das Wirgefühl und die Solidarität innerhalb einer Gruppe aufgrund geteilter Weltanschauungen und Werte und ein daraus resultierendes kameradschaftliches Füreinander-Eintreten im Mittelpunkt.

5) Engagement aufgrund des Bedürfnisses nach Hilfeleistung, Mitgestaltung und Verbesserung vorgefundener (Lebens-)Situationen. Zentral ist die subjektive Erwartung, mit den eigenen Kompetenzen etwas bewegen, unliebsame Dinge verändern und die Lebensqualität anderer Menschen verbessern zu können.

Engagements aus religiösen Wertüberzeugungen versus Profitorientierung und Optimierungswünsche

In Bezug auf die Unterteilung der MSO in zwei Gruppen (religiös, säkular) belegen signifikante Ergebnisse des $U$-Tests die größere Bedeutung der drei Motive „,religiöse Werte/Gebote umsetzen“, ,, anderen den Glauben nahebringen“ sowie ,mit Gleichgesinnten zusammenzukommen“ in religiösen Vereinigungen. In säkularen MSO besitzen hingegen die Erwartung von „Gegenleistungen“ und der Wunsch „Dinge verändern bzw. etwas bewegen zu wollen“ ein größeres Gewicht (Tab. 7.18).

Die jeweils höher eingeschätzten Motivbedeutungen spiegeln die unterschiedlichen Selbstverständnisse der beiden MSO-Typen und die in ihnen verschieden

Tab. 7.18 $U$-Test ${ }^{39}$ : Ergebnisse für fünf Motive (Gruppeneinteilung ,religiös/nichtreligiös“)

\begin{tabular}{|c|c|c|c|c|c|}
\hline$U$-Test & $\begin{array}{c}\text { Religiöse } \\
\text { Werte/Gebote } \\
\text { umsetzen }\end{array}$ & $\begin{array}{c}\text { Anderen } \\
\text { den Glauben } \\
\text { nahebringen }\end{array}$ & $\begin{array}{l}\text { Gegen- } \\
\text { leistung } \\
\text { erhalten }\end{array}$ & $\begin{array}{l}\text { Dinge } \\
\text { verändern, } \\
\text { verbessern } \\
\text { wollen }\end{array}$ & $\begin{array}{l}\text { Mit Gleich- } \\
\text { gesinnten } \\
\text { zusammen- } \\
\text { kommen }\end{array}$ \\
\hline$U$-Wert & 914 & 1281 & 2836 & 3475 & 3880 \\
\hline$z$-Wert & $-10,45$ & $-9,29$ & $-4,06$ & $-3,02$ & $-2,49$ \\
\hline Median $_{\mathrm{S}}$ & 4 & 4 & 3 & 1 & 2 \\
\hline Median $_{R}$ & 1 & 2 & 4 & 2 & 2 \\
\hline \multirow[b]{2}{*}{ Effektstärke $r$} & $0,72^{\star * *}$ & $0,65^{\star * *}$ & $0,29^{* * *}$ & $0,21^{* *}$ & $0,17^{*}$ \\
\hline & $\begin{array}{c}\text { Starker } \\
\text { Effekt }\end{array}$ & $\begin{array}{l}\text { Starker } \\
\text { Effekt }\end{array}$ & $\begin{array}{l}\text { Kleiner } \\
\text { Effekt }\end{array}$ & $\begin{array}{l}\text { Kleiner } \\
\text { Effekt }\end{array}$ & $\begin{array}{c}\text { Kleiner } \\
\text { Effekt }\end{array}$ \\
\hline
\end{tabular}

${ }^{39} \underline{U}$-Test: Berichtet wird die asymptotische Signifikanz (2-seitig) $\left(\mathrm{n}_{\mathrm{S}}+\mathrm{n}_{\mathrm{R}}>30\right)$.

Interpretation der Höhe der Effektstärken $r$ nach Cohen (1988).

Signifikanzniveaus $p$ : $* * *<0,001 ; * *<0,01 ; *<0,05 ;$ n. s. $=$ nicht signifikant. 
gelagerten Zweckorientierungen: Engagements aus religiösen, in der Gruppe geteilten Wertüberzeugungen stehen Engagements für einen konkreten Nutzen (Profit) und aufgrund des Interesses an der Optimierung bestimmter Zustände gegenüber.

\section{- Hypothesenprïfung und Ergebnisdiskussion}

Angesichts der obigen Befunde lassen sich die zwei Hypothesen zu in religiösen und säkularen MSO bedeutsamen Motiven nur teilweise bestätigen. Die Aussagen der Doppelhypothese lauten wie folgt:

H3 $_{1}$ : Aus Sicht der befragten Funktionsträger rangieren bei den Freiwilligen in religiösen MSO religiöse Wertüberzeugungen (anderen den Glauben nahebringen; religiöse Werte/Gebote umsetzen), gruppenbezogene Solidarität (mit Gleichgesinnten zusammenkommen; Solidarität mit der Organisation) und Hilfeleistung für andere vor anderen nutzen- und selbstbezogenen Motiven (Erwerb von Qualifikationen; Erhalt von Gegenleistung).

$\mathbf{H 3}_{2}$ : Die Motive der Freiwilligen, sich in nicht-religiösen MSO zu engagieren, bestehen nach Einschätzung der befragten Funktionsträger mit größerer Wahrscheinlichkeit aus selbstbezogenen Nutzenerwägungen (Erwerb von Qualifikationen; Erhalt von Gegenleistung), aus der Zuteilwerdung von Wertschätzung bzw. Anerkennung sowie aus der genussbetonten Bereicherung des eigenen Lebens (Freude haben).

Die obigen Analysen haben gezeigt, dass sich statistisch signifikante Unterschiede in Bezug auf die beiden religiösen Motive sowie das Bedürfnis der Zusammenkunft mit Gleichgesinnten ergeben. Die mit diesen Motiven theoretisch verquickten und für religiöse MSO ebenfalls als bedeutsamer geltend gemachten zwei Aspekte der Hilfeleistung sowie Solidarität mit der Organisation lassen sich jedoch nicht bestätigen. Wie erwartet besitzt allerdings der Erhalt von Gegenleistungen in religiösen MSO - wie auch bereits bei den Strategien zur Mobilisierung von Engagierten - aufgrund der dort prioritär wirksamen immateriellen Anreize einen statistisch signifikanten geringeren Stellenwert.

Das Motiv „Dinge verändern, etwas bewegen wollen“ wurde im Rahmen der theoretischen Unterstellungen (Doppelhypothese) nicht berücksichtigt. Es zeigen sich aber signifikante Testergebnisse: Für in nicht-religiösen MSO engagierte Personen wird diesem Aspekt eine größere Bedeutung beigemessen. Damit stellt es ein Unterscheidungskriterium dar, das auf die Ausrichtung und die Verfolgung konkreter Zwecke der Organisationen zurückzuführen sein wird, bei denen proaktives Verhalten eine wichtige Rolle spielt.

Ebenfalls nicht bekräftigen lassen sich die in $\mathbf{H 3}_{\mathbf{2}}$ verarbeiteten theoretischen Annahmen eines in säkularen MSO registrierbaren größeren Gewichtes der mit dem Engagement einhergehenden Erwartung von Wertschätzung bzw. Anerkennung 
sowie des Freudehabens. Vielmehr werden die zwei Dimensionen ohne signifikante Unterschiede in religiösen und nicht-religiösen MSO für die Engagierten als relevant eingestuft, womit sich die Organisationen folglich in Bezug auf diesbezügliche Bedürfnisbefriedigungen ähneln. Theoretisch war davon ausgegangen worden, dass sich der Aspekt einer persönlichen Bestätigung durch Fremdachtung (Anerkennung) als mit einem konkreten selbstbezogenen Nutzen (Gegenleistung, Qualifizierung) verkoppelt zu betrachten ist, was sich anhand der EFA auch als zutreffend erweist (Faktor 3), nicht aber in Bezug auf die mit $\mathrm{H}_{2}{ }_{2}$ im nicht-religiösen Organisationskontext ins Visier genommenen Engagierten.

In Bezug auf die Frage nach der Freude an der Tätigkeit könnte argumentiert werden, dass diese abkömmlich ist, da ihre Bejahung zu erwarten ist: Welche Person wird freudlos freiwillig und unbezahlt eine Tätigkeit übernehmen? Zudem ist nicht auszuschließen, dass die Funktionsträger dieses Motiv für zutreffend erklären, da es ihnen wichtig ist, dass die Engagierten in der MSO Spaß haben oder weil sie sich in der Verantwortung sehen, dass die dortigen Rahmenbedingungen Spaß ermöglichen. Davon unabhängig kann allerdings andererseits nicht automatisch angenommen werden, dass ein Engagement im Organisationskontext, auf kollektiver Ebene, zwangsläufig oder in erster Linie mit persönlichem Vergnügen in Verbindung steht. Stattdessen könnten etwa auch das Gefühl einer moralischen Verpflichtung oder der Aufforderungscharakter religiöser Gebote, andere Menschen zu unterstützen, dominieren (II. 4.3.3.2). Darüber hinaus ist der Aspekt des Spaßhabens im Kontext der Werteforschung zu betrachten, denn diese verweist auf zwischen bestimmten Werthaltungen bestehende positive und negative Korrelationen: So können empirisch enge positive Zusammenhänge zwischen religiösen Werten und den Wertorientierungen der Traditionalität, Sicherheit, Konformität, Anpassung und Selbstkontrolle und negative Korrelationen mit denjenigen der Selbstentfaltung, Risikobereitschaft und des Hedonismus festgestellt werden (Schwartz/Huismans 1995; Pickel 2018: 969 ff.; II. 4.3.3.2). Dieses bedeutet jedoch nicht, dass diese konträren Werthaltungen (in Anbetracht des Wertewandels) nicht auch miteinander gekoppelt und im weiteren Lebensverlauf bedeutsam sein können (ebd., vgl. auch Pickel/Krüggeler 2001; Parsons 1977: 279-320).

Für die im deutschen Kontext im Interessenfokus stehenden und in der auszuwertenden Stichprobe dominant abgebildeten Muslime bzw. islamischen Organisationen ist allerdings zu konstatieren, dass angesichts diverser Einflussfaktoren und Erklärungsmodelle unter Bedingungen kultureller Differenz potenziell sehr unterschiedliche Wertorientierungskombinationen und Wertrichtungen möglich sind (Diehl/Koenig 2009; Nauck 2007; Ceylan 2012a; Nagel 2018a, b). So zeigen jüngere personenbezogene Studien für Muslime zum einen eine stärkere Traditionsverhaftung und Sicherheitsorientierung als bei Christen und zum anderen sowohl bei 
jüngeren und älteren Altersklassen eine hohe Bedeutung der Werte Hilfsbereitschaft und Hedonismus (Pollack/Müller 2013: 30, 65; Sinnemann 2017: 29, 44; II. 4.3.3.2). Auch hinsichtlich der Motive von engagierten Muslimen sind anhand der Daten des Freiwilligensurveys 2014 im Vergleich zu anderen Konfessionen Abweichungen auffällig, insofern muslimische Engagierte bei mit einem persönlichen (im-)materiellen Nutzen verknüpften Motiven leicht bis stark erhöhte Werte aufwei$\operatorname{sen}^{40}$ (Sinnemann 2017: 29 f.). Damit ist eine durchaus mögliche Kompatibilität von traditionell-konservativen und individualistisch-hedonistischen Wertvorstellungen angezeigt. Dieser Befund korrespondiert mit dem Ergebnis, dass persönliche Vergnügung für in religiösen MSO Engagierte als nicht weniger wichtig erachtet wird. In diesem Zusammenhang künftig mehr Aufmerksamkeit erhalten sollte der durch soziale Beziehungen und eine gemeinsame Tätigkeitsausübung Konvivialität und Spaß ermöglichende Organisationskontext.

Diese Gesichtspunkte werden - im Gegensatz zu Engagementuntersuchungen auf der Individualebene - in Anreiz-Beitrags-Konzeptionen, aber auch in Organisationstypologien und -theorien marginalisiert und insbesondere in Hinblick auf religiöse bzw. weltanschauliche Organisationen weitestgehend ausgeblendet (II. 4.4.1; vgl. Clark/Wilson 1961; Barnard 1970; Simon 1981/[1945]; March/Simon 1976/[1958]; Parsons 1960; Etzioni 1975; Blau/Scott 1962; Beckford 1975; Demerath et al. 1998; Sanders/Kianty 2006; Scott/Davis 2007; Petzke/Tyrell 2012; Kühl 2015; Wilson 2020). Stattdessen wird die Verinnerlichung von handlungsregulierenden religiösen Normen, die Konformität mit moralischen Vorschriften und Verpflichtungen und die Relevanz von (sozialen) Kontrollmitteln für die Einhaltung der religiösen Normen und die Gewährleistung des Gruppenzusammenhalts unterstrichen (vgl. etwa Etzioni 1975: 40 ff.; siehe zu diesem Befund auch 7.3: $>$ Fazit und $\$ Ausblicke).

Kompatibilität von religiösen und nicht-religiösen Motivstrukturen

In der Summe zeigen die Ergebnisse, dass nicht von einer theoretisch naheliegenden und geradlinigen Verkettung von jeweils säkular und religiös verankerten Motiven auszugehen ist. Die praktische Realität erweist sich als komplexer und das Verhältnis von religiösen zu anderen Werten ist offener und flexibler zu denken. Die Tatsache, dass die sehr eng gefasste Doppelhypothese als solche empirisch nicht bestätigt werden kann, legt nahe, dass gruppenbezogene religiöse Wertüberzeugungen sowie geselligkeits-, nutzen- und selbstbezogene Motive

\footnotetext{
${ }^{40}$ Dies gilt auch für Evangelisch-Freikirchliche und ist vermutlich v. a. auf die in beiden Gruppen vertretenen jüngeren Altersgruppen zurückzuführen.
} 
gut miteinander kompatibel und mit den Zielsetzungen, Interessen und Selbstverständnissen der MSO vereinbar sein können. Abgesehen von den im Rahmen dieser Organisationsbefragung hervorgetretenen Differenzen in Bezug auf Religiosität, Profitabilität und (Um-)Gestaltungsimpetus scheinen sich religiöse und säkulare MSO hinsichtlich ihrer Verträglichkeit mit den theoretisch als einander ausschließend betrachteten Wertorientierungen bzw. Motiven gar nicht so sehr voneinander zu unterscheiden.

Das im Organisationskontext durchaus mögliche Zusammenspiel von religiösen und nicht-religiösen Motiven lässt sich auch anhand von in Wohlfahrtsverbänden und MSO durchgeführten Engagierten-Befragungen nachweisen (Klöckner 2016). Jennifer Klöckner zeigt auf, dass religiöse Motive von den Engagierten in Kombination mit sozialen und kulturellen Aspekten genannt werden. Die Autorin schlussfolgert, dass die Konfessionszugehörigkeit mithin nicht als alleiniger Grund für die freiwillige Arbeit in religiösen Vereinen gewertet werden kann, sondern dass sich insbesondere in den religiösen Beweggründen von in türkisch-islamischen Vereinen engagierten Personen zugleich das Bedürfnis der Bewahrung der Sprache und Kultur aus dem Herkunftsland sowie der Kontaktpflege zur eigenen ethnischen Community manifestiere (ebd.: 434 ff.; vgl. auch Handy/Greenspan 2009: 957; Carabain/Bekkers 2010: 3). In den Ergebnissen deutet sich an, dass den MSO multiple Funktionen zuzuweisen sind, was in der Forschung insbesondere für muslimische Moscheevereine herausgestellt wird (Ceylan 2006: 123 ff.; Ceylan 2008; Behloul/Lathion 2007: 201; Baumann 2016: 111).

Insgesamt ist also festzuhalten, dass das Engagement in religiösen Organisationen nicht vorschnell und ausschließlich auf religiöse Faktoren zurückgeführt werden sollte, sondern grundsätzlich von Motivbündeln und vielfältigen, gemischten Anreizen auszugehen ist.

\subsubsection{Die MSO:Ziele, Selbstverständnisse, soziales Miteinander}

\section{Ziele der MSO}

Die Ziele einer Organisation sind für deren Gründungsprozess, Bestandserhaltung, Verhaltensweisen und die Motivation der Mitglieder wichtig. Denn ohne ein „Ziel für die Zusammenarbeit [...] weiß niemand, welche Leistungen von den einzelnen verlangt werden müssen und welche Befriedigungen ihnen geboten werden können“ (Barnard 1970: 81). Da die Mitglieder in freiwilligen Vereinigungen ein genuines Interesse an den Vereinszielen als Leitsätze für die Vereinstätigkeiten haben, sind 
Mitgliederinteressen und Ziele in idealtypischer Hinsicht deckungsgleich (II. 4.3.1; II. 4.3.3).

Die Ziele der befragten MSO sind vielschichtig. Die Ergebnisse zeigen insgesamt das über spezifische Gruppeninteressen hinausgehende Anliegen, sich für das allgemeine Wohl und Belange des Gemeinwesens bzw. der Gesellschaft in Deutschland einzusetzen ( $89 \%$ ); zugleich zeigt sich eine hohe Solidarität mit und Unterstützung von Zugewanderten und Landsleuten $(87 \%)$, deren Lebenssituation und Teilhabe in Deutschland durch die Aktivitäten der MSO verbessert werden sollen. Dies betrifft auch die Unterstützung von sozial benachteiligten und schwachen Mitgliedern (77\%) (Abb. 7.7).

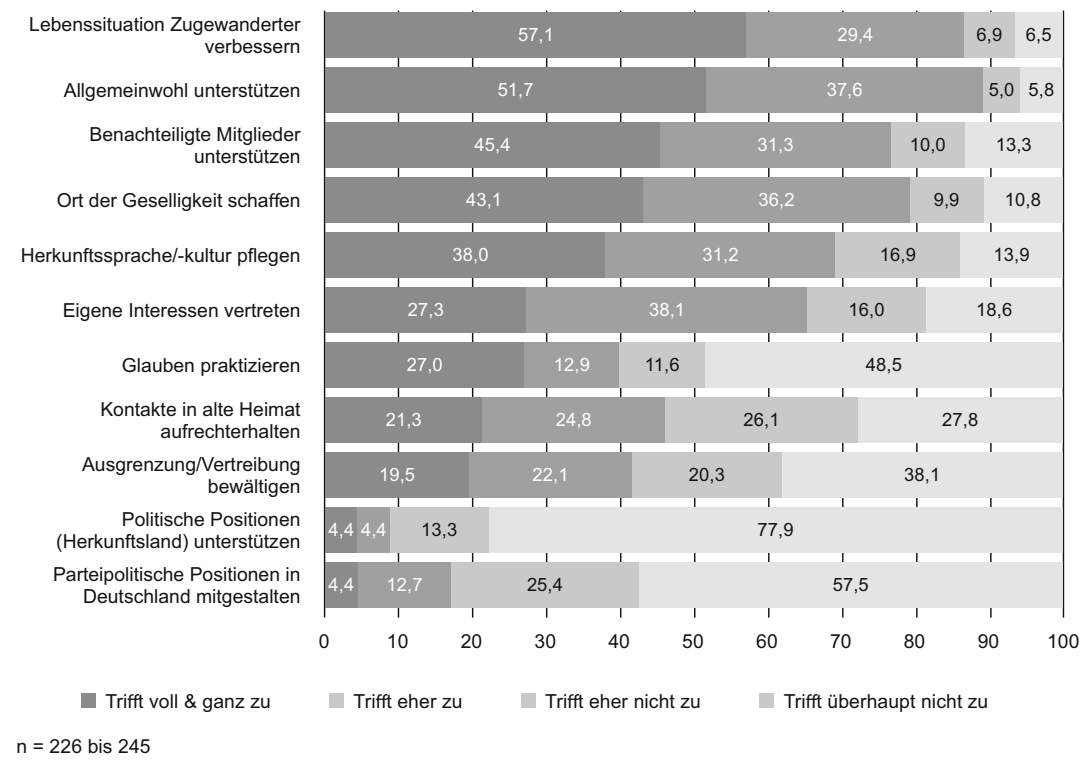

Abb.7.7 Ziele der MSO (in \%)

Eine ebenfalls hohe Relevanz kommt den Selbstorganisationen als Orte der Geselligkeit und Zusammenkunft zu (79\%). Dieser Aspekt erhält seitens der religiösen Vereinigungen einen um 14 Prozentpunkte höher liegenden Zustimmungswert (RMO: $89 \%$ vs. MO: $75 \%$ ). 
Die Bewahrung und Pflege der eigenen Herkunftssprache und -kultur in Deutschland (69\%) scheint für alle MSO eine deutlich größere Bedeutung zu besitzen als die Aufrechterhaltung von Kontakten in die alte Heimat (46\%). Ein deutlich geringerer Stellenwert kommt in den Vereinigungen auch der Bewältigung der Erfahrung von Ausgrenzung und Vertreibung im Herkunftsland zu (42\%).

Eine weiterhin festzustellende Bedeutsamkeit der Vertretung und Sichtbarmachung von eigenen Interessen (65\%) korrespondiert jedoch nicht mit der Absicht, parteipolitische Positionen in Deutschland mitzugestalten bzw. zu beeinflussen $(17 \%)$ und noch weniger mit dem Anliegen, im Herkunftsland vertretene politische Positionen in Deutschland zu unterstützen (9\%).

Die gemeinsame Praxis des religiösen Glaubens spielt mit 40 Prozent ebenfalls eine Rolle, und zwar insbesondere für die religiösen Vereinigungen (96\%), aber mit rund 12 Prozent auch in nicht-religiösen MSO.

13 Prozent - und zwar fast ausschließlich nicht-religiöse MSO - führten unter „Sonstiges“ weitere Ziele an. Unter diesen wurden mehrmals die Förderung der Integration von Migranten (in den Arbeitsmarkt und im Bildungsbereich) und des interkulturellen Austausches, die Leistung von Entwicklungshilfe im Herkunftsland (in Afrika), aber auch vereinzelte Anliegen wie die Hilfeleistung für Straßentiere oder der Zweck, lediglich ein Fanclub sein zu wollen, vermerkt.

\section{- Statistische Tests, Hypothesenprüfung und Ergebnisinterpretation}

Religiöse MSO als gesellige und soziale Orte

Ergebnisse des $U$-Tests zeigen, dass sich die beiden Organisationstypen hinsichtlich der zwei Zwecke eines geselligen Beisammenseins sowie der gemeinsamen Glaubenspraxis signifikant unterscheiden - und zwar kommt beiden Aspekten in den religiösen Vereinigungen eine größere Bedeutung $\mathrm{zu}$, was insbesondere für die Religionsausübung zutrifft. ${ }^{41}$ Damit untermauern die statistischen Testergebnisse die Aussage, dass die religiösen MSO religiöse und soziale Orte zugleich sind, an denen das soziale Eingebundensein auch jenseits religiöser Zeremonien und weiterer religiöser Bedürfnisbefriedigungen eine bedeutende Rolle spielt. Sie korrespondieren damit auch mit den oben diskutierten Motivbefunden, die ebenfalls verdeutlichen, dass religiöse MSO Plattformen sozialer Beziehungen sind, an denen nicht nur gemeinsam der religiösen Brauchtumspflege nachgegangen wird,

${ }^{41}$ Einen Ort der Geselligkeit und Zusammenkunft schaffen $\underline{U}$-Test: $U=4821,5 ; z=$ $-2,22 ; p=0,027 ; r=0,15 ; \mathrm{Md}_{\mathrm{S}}=2 ; \mathrm{Md}_{\mathrm{R}}=1$ - Den religiösen Glauben gemeinsam praktizieren $\underline{U}$-Test: $U=616,5 ; z=-12,04 ; p=0,000 ; r=0,79 ; \mathrm{Md}_{\mathrm{S}}=4 ; \mathrm{Md}_{\mathrm{R}}=1$. 
sondern auch ein zwangloses Beisammensein für angenehmen Zeitvertreib und Vergnügung stattfinden kann. Hingegen ist den säkularen MSO demnach eher eine professionellere Ausrichtung zu attestieren.

Die Zusammenschau der Organisationsziele zeigt, dass die an dem Konzept der Binnenintegration (II. 4.1) ansetzende, auf alle MSO bezogene Hypothese nur bedingt bestätigt werden kann. Sie lautet:

H1: Verglichen mit anderen Zwecken stellen die Aufrechterhaltung der Herkunftskultur und die Schaffung eines Ortes der Sozialität sowohl für nichtreligiöse als auch religiöse MSO besonders wichtige Organisationsziele dar.

Ihr Ausgangspunkt bildet die Annahme, dass migrantische Vereinigungen für die involvierten Individuen essenzielle identitäts- und persönlichkeitsstabilisierende Funktionen übernehmen. Dies ist darauf zurückzuführen, dass die Binnengruppe die Aufrechterhaltung vertrauter herkunftslandbezogener Verhältnisse und Praktiken, intraethnische Kontaktpflege und Geselligkeit ermöglicht (Elwert 1982; II. 4.1). Angesichts der Befragungsergebnisse kann allerdings nicht davon ausgegangen werden, dass die hervorgehobenen Aspekte der Pflege der Herkunftskultur und des sozialen Miteinanders in den MSO tonangebend sind: Sie sind zwar bedeutsam, rangieren aber nicht an erster, sondern an vierter und fünfter Stelle (volle Zustimmung) im zweiten obersten Drittel hinter den auf Nachteilsausgleich von Gruppenmitgliedern und gesellschaftliche Mitgestaltung ausgerichteten Zielen (Abb. 7.7). Hier zeigt sich, dass sich die theoretischen Annahmen in der Realität zwar als relevant erweisen, aber alleinig - anhand der zwei Items - fokussiert zu kurz greifen und unzeitgemäß sind. Denn im Generationenverlauf, mit fortdauerndem Aufenthalt, Geburt und Sozialisation in Deutschland verstärken sich die Identifikation mit dem Heimatund Lebensort und Bedürfnisse, sich für allgemeine gesellschaftliche Interessen und Angelegenheiten einzusetzen (BMFSFJ 2017: 200 f.; Halm/Amberge 2015). Dies schließt sowohl das selbstbewusste Einfordern chancengleicher Teilhabe als auch das Ausleben kultureller Prägungen in der eigenen Community ein.

Höhere Relevanz von gesellschaftlicher Mitgestaltung und der Bewältigung sozialer Ausgrenzungserfahrungen für jüngere MSO

Die Anwendung des Rangkorrelationskoeffizienten nach Spearman zeigt für das Organisationsalter und drei Organisationziele signifikante Korrelationen. Bei den drei Zielen handelt es sich um das Tätigsein für das Gemeinwohl in Deutschland, die Bewältigung von im Herkunftsland erfahrener Ausgrenzung und Vertreibung sowie die gemeinsame Glaubenspraxis. 
Während sich für die zwei erstgenannten Ziele das Verhältnis zeigt: Je jünger eine MSO ist, desto höher fällt die Bedeutung der Zwecke aus, lässt sich für das letztgenannte Ziel ermitteln, dass die Relevanz der Glaubenspraxis abnimmt, je jünger die MSO ist. ${ }^{42}$ Dies korrespondiert mit dem Befund, dass die religiösen MSO im Durchschnitt älter sind (7.1.1). Damit zeigt sich, dass gesellschaftliche Mitgestaltung wie auch die Verarbeitung von im Herkunftsland kollektiv erfahrener Diskriminierung in Deutschland für die jüngeren Vereinigungen und Generationen von größerer Bedeutung und Aktualität sind. Der höher ausgeprägte Verarbeitungsbedarf sollte auch auf nicht lange zurückliegende Erlebnisse in den Herkunftsländern zurückzuführen sein, aufgrund derer die ursprünglichen Heimatorte verlassen wurden.

Darüber hinaus verdeutlichen die Ergebnisse einer EFA, dass die beiden Items (,Ort der Geselligkeit schaffen“ und ,Herkunftskultur aufrechterhalten ') mit anderen Organisationszielen verknüpft sind (Tab. 7.19; Kursivsetzungen). Der Faktorladungsmatrix zufolge lassen sich die Ziele der MSO in drei Faktoren konzentrieren und umfassender benennen:

1) Nachteilsausgleichende und gemeinwohlorientierte Vergemeinschaftung.

Die Verbesserung bzw. Stabilisierung der Lage zugewanderter Landsleute und Organisationsmitglieder basiert auf einem Gemeinschaftsgefühl und geht mit gesellschaftlichen Beteiligungsanliegen einher.

2) (Politische) Interessenvertretung und Teilhabe in Deutschland.

Die Anliegen von (politischer) Beteiligung, Verarbeitung erfahrener Ausgrenzung und Interessenvertretung finden im Spannungsfeld von Herkunftsland und Aufnahmegesellschaft statt.

3) Herkunftsland- und Religionsbezogenheit.

Die Kontakte zur Herkunftskultur, ihrer Pflege wie auch die gemeinsame Religionsausübung indizieren eine Bezogenheit auf die eigene Gruppe und eine Konsolidierung nach innen.

\footnotetext{
${ }^{42}$ Etwas für das allgemeine Wohl in Deutschland tun: rho $=-0,16^{*}$ - Erfahrung von Ausgrenzung/Vertreibung im Herkunftsland in Deutschland bewältigen: $r h o=-0,17 * *$ - Den religiösen Glauben gemeinsam praktizieren: $r h o=0,21 * *$ (Anmerkung: Aufgrund der inversen Codierungen der ordinalskalierten Variablen zeigt ein negatives Vorzeichen von Spearman-Rho hier einen positiven Zusammenhang mit dem Organisationsalter an).
} 
Tab. 7.19 Rotierte Faktorladungsmatrix zu Organisationszielen

\begin{tabular}{|c|c|c|c|}
\hline & \multicolumn{3}{|c|}{ Faktoren } \\
\hline & 1 & 2 & 3 \\
\hline $\begin{array}{l}\text { Die Lebenssituation und Teilhabe von Zugewanderten/ } \\
\text { Landsleuten in Deutschland verbessern }\end{array}$ & ,808 & ,129 & ,068 \\
\hline Etwas für das allgemeine Wohl in Deutschland tun & ,768 & 037 & , 148 \\
\hline Sozial benachteiligte und schwache Mitglieder unterstützen & ,690 & , 182 &,- 065 \\
\hline Einen Ort der Geselligkeit und der Zusammenkunft schaffen &,$\underline{564}$ &,- 019 & $\underline{\underline{526}}$ \\
\hline $\begin{array}{l}\text { Im Herkunftsland vertretene politische Positionen in Deutschland } \\
\text { unterstützen }\end{array}$ &,- 118 & ,746 & ,163 \\
\hline Parteipolitische Positionen in Deutschland mitgestalten/beeinflussen & ,135 & ,726 &, 079 \\
\hline $\begin{array}{l}\text { Die Erfahrung von Ausgrenzung und Vertreibung im Herkunftsland } \\
\text { in Deutschland bewältigen }\end{array}$ & ,454 & ,619 &,- 061 \\
\hline Eigene Interessen vertreten und sichtbar machen & ,387 &, 505 & , 137 \\
\hline $\begin{array}{l}\text { Die eigene Herkunftssprache und -kultur in Deutschland aufrecht- } \\
\text { erhalten und pflegen }\end{array}$ & ,279 &, 110 & ,773 \\
\hline Kontakte in die alte Heimat aufrechterhalten & ,058 & ,457 & ,653 \\
\hline Den religiösen Glauben gemeinsam praktizieren &,- 103 & 020 & ,638 \\
\hline
\end{tabular}

Hauptkomponentenanalyse. Fehlende Werte: paarweiser Fallausschluss. Rotationsmethode: Varimax. KMO-Test: 0,$755 ; \mathrm{n}=226-245$.

„Geselligkeit“ als zentrales Scharnier zwischen binnen- und außenorientierter Integration

Die Querladung des Organisationsziels „Einen Ort der Geselligkeit und Zusammenkunft schaffen“ auf die Faktoren 1 und 3 lässt erkennen, dass es (inhaltlich) mit Items korreliert, die auf den zwei identifizierten Faktoren laden. Da die Ladung größer als 0,5 ist, ist die Variable zur Interpretation beider Faktoren heranzuziehen (Backhaus et al. 2018: 399). Die Überlappung verweist auf den theoretisch durchaus plausiblen Zusammenhang, dass der Aspekt der Kontaktpflege und Geselligkeit innerhalb einer Organisation nicht dem einen oder anderen Faktor eindeutig zuzuschreiben ist, sondern für beide Relevanz besitzt. So ist das soziale Miteinander in der Gruppe zum einen als ein wichtiger kohäsions- und konsolidierungsfördernder Faktor und damit als ein ermöglichendes Fundament für die Verbesserung der Lebenssituationen und Unterstützung von benachteiligten Mitgliedern, die ggf. von gesellschaftlicher Marginalisierung bedroht sind, zu betrachten (Faktor 1). Der Geselligkeitsaspekt kann durch die Gruppenkohäsion und binnenorientierte Aktivitäten (Faktor 3) Erfahrungen von Eigenstärke, Autonomie und Kompetenz bewirken und dadurch zum anderen auch Kräfte freisetzen, die die Gruppe zur außenorientierten Beteiligung insbesondere in Minderheitensituationen animieren und befähigen (Faktor 1) (vgl. dazu auch Elwert 
1982; Uslucan 2015, 2017a; II. 4.1). Insofern ist hier von einem interdependenten, integrativen Wirkungsgefüge mit Bezügen sowohl zum Herkunftsland als auch zur Aufnahmegesellschaft auszugehen und dem Wunsch nach sozialen Beziehungen, Interaktionen und Vergemeinschaftung (über die Selbstorganisation) folglich eine zentrale Scharnierfunktion beizumessen. Diese gruppenstabilisierende Scharnierfunktion kann die Gruppe sowohl nach innen als auch nach außen integrieren, ,Zwischen Vergemeinschaftung und Vergesellschaftung“ (Pries 2010a: 37; II. 4.1) vermitteln und mithin wichtige Integrationsfunktionen übernehmen. Positive Wirkungen der Geselligkeits- und Binnenorientierung können an gesamtgesellschaftliche Belange angedockt werden bzw. für die Außenorientierung und Vergesellschaftung nutzbar sein. Die Soziabilität kann damit als ein Bindeglied zwischen gruppenbindendem und -überbrückendem Sozialkapital fungieren bzw. als ein Einfallstor für die Herstellung flexibler Übergänge und Verbindungen betrachtet werden. Das eigenkulturelle Sozialkapital sollte daher als ein wichtiger Faktor gesellschaftlicher Integration nicht relativiert werden.

Angesichts dieser Erörterung spricht die Querladung in der rotierten Komponentenmatrix für eine Bekräftigung einer sich positiv auswirkenden Binnenintegration und - auf den zweiten Blick - für eine Bestätigung des sich auf die Sozialität als ein zentrales Organisationsziel beziehenden Teils der Hypothese H1. Diese wurde im Kontext der Annahmen von Friedrich Heckmann (1992), Georg Elwert (1982) und Hartmut Esser (1986a) formuliert (II. 4.1), die alle die identitäts- und persönlichkeitsstabilisierenden Funktionen herkunftslandbezogener Verhältnisse und intraethnischer Kontaktpflege betont haben. Elwert zufolge sind die mit der Binnenintegration einhergehende Markierung und Bewahrung einer eigenständigen kulturellen Identität durchaus mit Kontaktaufnahmen zur Mehrheitsgesellschaft (der „Außenwelt") und Prozessen der Akkulturation kompatibel (ebd.: 726). Das bedeutet, dass eine starke Integration von Einwanderern innerhalb eigenethnischer Sozialstrukturen ,unter bestimmten Bedingungen ein positiver Faktor für ihre Integration in eine aufnehmende Gesellschaft" (Elwert 1982: 718; Herv.: d. Verf.) sein kann (II. 4.1). Eine wichtige Voraussetzung dafür ist jedoch, dass sich an bestimmte (religiöse, ethnische) Identitäten und Zugehörigkeiten gebundene Individuen, die intensiv in ihrer Gemeinschaft aktiv sind, der Existenz und Bedeutsamkeit eines größeren Gemeinschaftslebens sowie eines allgemeinen gesellschaftlichen Wohls bewusst werden sollten. Dies impliziert die Durchführung gemeinsamer gruppenübergreifender Aktivitäten, die auf die Verwirklichung von gesellschaftlich als wichtig und nutzbringend erachteten Zielen gerichtet sind. Die Teilnahme an den Aktivitäten der eigen(ethnisch)en Gruppe und die Unterstützung der von ihr vertretenen Werte müssen daher flankiert werden von dem Bewusstsein, Teil einer Gesellschaft mit gemeinsamen Interessen und Gütern zu 
sein. Die Möglichkeiten der Gruppenmitglieder müssen dahingehend freigesetzt werden, was wiederum Interaktionen und Verbindungen mit anderen Gruppen bzw. die Befähigung und Bereitschaft solche einzugehen voraussetzt.

Der im Rahmen dieser Arbeit durchgeführten Befragung zufolge sind die vielfältigen Ziele der MSO dominant auf Deutschland und die Verbesserung der Situation von Landsleuten bzw. Menschen mit einer Zuwanderungsgeschichte ausgerichtet, aber auch auf die kollektive, soziale Ebene der gesellschaftlichen Umwelt als eine wichtige Bedingung für ein gelingendes Zusammenleben. ${ }^{43}$ Werden diese Ziele ernst genommen bzw. realiter umgesetzt, dann sind die MSO potenziell imstande, bedeutende Brückenfunktionen zu übernehmen, indem Belange sowohl der Minderheits- als auch der Mehrheitsgesellschaft ins Visier genommen werden und zwischen unterschiedlichen Ausgangspositionen und Bedürfnislagen vermittelt wird. Dies kann mit durchaus selbstverstärkenden Effekten einhergehen, wenn sich die Gruppenstärke positiv auf deren Aktivitäten in der Mehrheitsgesellschaft auswirkt und dies wiederum zu einer weiteren Selbstvergewisserung und Konsolidierung der Mitglieder und ihrer Organisationen führt.

Wie auch in Vereinigungen von Menschen ohne Migrationshintergrund finden sich in den MSO mit spezifischen Interessen verbundene Zwecke: Heimatpflege, Sozialkontakte, gemeinsame Glaubenspraxis, Artikulation und Sichtbarmachung gruppenspezifischer Anliegen. Letzteres kann aber auch lediglich den Wunsch nach Erhalt von Aufmerksamkeit und Beachtung auf unterschiedlichen gesellschaftspolitischen Ebenen beinhalten, ohne zwangsläufig das Bedürfnis zu umfassen, durch aktiven Einsatz etwas verändern zu wollen. Auch dürften einige MSO lieber unter sich bleiben und nicht in der Öffentlichkeit stehen wollen, was auch für viele Vereine von Menschen ohne Migrationshintergrund in Deutschland geltend gemacht werden kann (BMFSFJ 2017: 75). Die Tatsache, dass sich die MSO sowohl in Bezug auf im Herkunfts- als auch in Deutschland existierende (partei-)politische Verhältnisse eher unpolitisch zeigen, deutet auch im Zusammenhang mit einer für weniger als die Hälfte der Vereinigungen relevanten Kontaktpflege in eine alte Heimat (von Vorfahren) auf eine weniger ausgeprägte Herkunftslandorientierung hin.

Vor diesem Hintergrund sind einzelne Befunde zu den Zielen als solche nicht vorschnell mit abgrenzenden oder Gruppenexklusivität beanspruchenden Tendenzen zu assoziieren (7.2).

\footnotetext{
${ }^{43}$ An dieser Stelle ist anzumerken, dass die Ziele von MSO nicht immer zwangsläufig menschliche Belange adressieren müssen, sondern beispielsweise auch den Schutz von Tieren betreffen können.
} 


\section{Selbstverständnisse der MSO}

Säkulare MSO als Netzwerke und Förderorganisationen, religiöse als Gemeinschaften Gleichgesinnter

Die Funktionsträger wurden gebeten, anhand von 13 Items das Selbstverständnis ihrer Organisation einzuschätzen. Dieses betrifft die Vorstellung, die die Organisationen von sich selbst haben und mit dem sie sich in der Öffentlichkeit präsentieren. Diese Selbstdefinitionen bzw. Selbstdarstellungen sollten die kollektiven Interessen spiegeln und damit auch die Ausgestaltung der Ziele und zentralen Aufgabenfelder einer Organisation.

In der Mehrzahl werden die MSO als Brückenbauer und Kulturdolmetscher (85\%) sowie Integrationspromotoren (82\%) aufgefasst (Abb. 7.8). Damit werden in der politischen Debatte für migrantische Vereinigungen stark gemachte Funktionen durch deren Selbstinterpretation quasi bestätigt. Die Frage, inwieweit sie diese Rollen tatsächlich ausfüllen, ist an anderer Stelle genauer unter die Lupe zu nehmen (7.2).

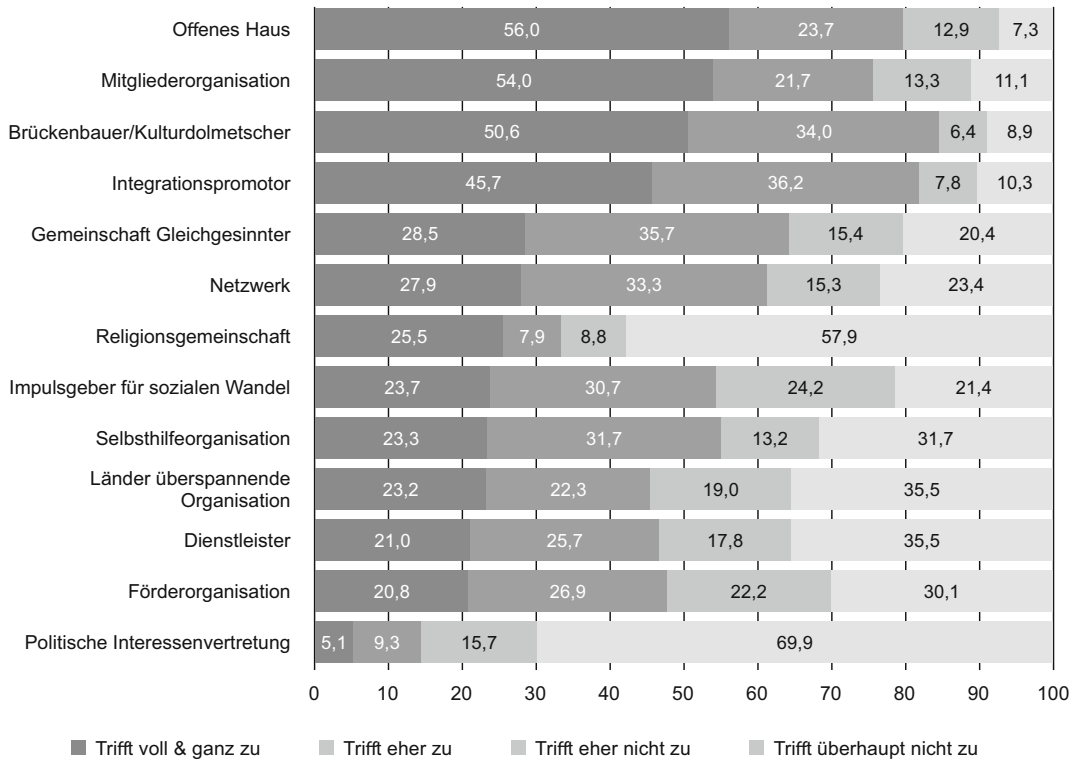

$\mathrm{n}=214$ bis 235

Abb. 7.8 Selbstverständnisse der MSO (in \%) 
Jeweils über drei Viertel der befragten MSO-Vertreter betrachten ihre Organisationen als ein offenes Haus, in dem jedwede Person willkommen ist (80\%), und als eine Mitgliederorganisation (76\%). 64 Prozent aller MSO werden als Gemeinschaften Gleichgesinnter charakterisiert, wobei diese Bezeichnung für die religiösen MSO öfters als zutreffend erachtet wird (76 \% vs. $58 \%$ ). Genau ein Drittel der MSO wird das Selbstverständnis einer Religionsgemeinschaft attribuiert $(33 \%)$. Darunter sind sinnfälliger Weise dominant religiöse MSO vertreten ( $84 \%)$, aber immerhin auch rund 6 Prozent der säkularen MSO. Letztere werden hingegen bei einer allgemeinen Zustimmungsrate von 61 Prozent um rund 25 Prozent häufiger als ein Netzwerk aufgefasst (MO: 69 \% vs. RMO: $44 \%$ ). Etwa im gleichen Verhältnis werden die MSO als Selbsthilfeorganisation (55\%) und Impulsgeber für sozialen Wandel (54\%) sowie von weniger als der Hälfte als Förderorganisationen eingeordnet ( $48 \%$ ). Diese Kategorie trifft wiederum häufiger für nicht-religiöse Vereinigungen zu (+16 Prozentpunkte). Als Dienstleister werden ebenfalls weniger als die Hälfe der MSO aufgefasst (47\%); gleiches gilt für die zwei Standpunkte, eine mehrere Länder überspannende Organisation (46\%) oder eine politische Interessenvertretung (Pressure-Group) darzustellen (14\%).

\section{- Statistische Tests und Ergebnisdiskussion}

Berechnungen des $U$-Tests ergeben hinsichtlich der Selbstdefinitionen, dass sich die religiösen Vereinigungen signifikant häufiger als Religionsgemeinschaften und Gemeinschaften Gleichgesinnter verstehen. Hingehen sind für die säkularen MSO die Selbstbilder eines Netzwerks und einer Förderorganisation signifikant bedeutsamer. ${ }^{44}$ Während die religiösen MSO damit die Relevanz von Verbundenheit und geteilten Werten innerhalb der eigenen Gemeinschaft betonen, akzentuieren die säkularen Vereinigungen ihr Interesse an Austausch und Verbindung mit anderen Akteuren sowie das Anliegen, Entwicklung und Vorankommen von Prozessen aktiv unterstützen zu wollen.

In Hinblick auf die anderen Selbstverständnisse (Selbsthilfeorganisation, politische Interessenvertretung, Integrationspromotor, Dienstleister etc.) zeigen sich keine signifikanten Diskrepanzen zwischen den (nicht-)religiösen Vereinigungen. Die Ergebnisse spiegeln exemplarisch die mehrfach verorteten Kollektividentitäten

\footnotetext{
${ }^{44}$ Religionsgemeinschaft $U$-Test: $U=570 ; z=-12,195 ; p=0,000 ; r=0,83 ; \mathrm{Md}_{\mathrm{S}}=4$; $\mathrm{Md}_{\mathrm{R}}=1$ - Gemeinschaft Gleichgesinnter $\underline{U}$-Test: $U=3997,5 ; z=-3,2 ; p=0,001$; $r=0,22 ; \mathrm{Md}_{\mathrm{S}}=2 ; \mathrm{Md}_{\mathrm{R}}=2$ (Anmerkung: Wie auch bei dem Motiv , um mit Gleichgesinnten zusammenzukommen“ liegt für die religiösen MSO ein niedrigerer gemittelter Rang vor, wodurch eine höhere Merkmalsausprägung angezeigt wird) - Netzwerk $U$-Test: $U=$ 3827,$5 ; z=-3,407 ; p=0,001 ; r=0,23 ; \mathrm{Md}_{\mathrm{S}}=2 ; \mathrm{Md}_{\mathrm{R}}=3$ - Förderorganisation $U$-Test: $U=4224 ; z=-2,133 ; p=0,033 ; r=0,15 ; \operatorname{Md}_{\mathrm{S}}=2 ; \mathrm{Md}_{\mathrm{R}}=3$.
} 
der Vereinigungen. Sowohl bei den religiösen als auch bei den säkularen Organisationen geht die Besinnung auf die eigene Gruppe mit gesellschaftsrelevanten Außenorientierungen einher, die auch ein selbstverpflichtendes Moment implizieren (wie es etwa die Ausübung von „dienstleistenden“, „fördernden“ Tätigkeiten einschließt).

Hinsichtlich des oben dargelegten Antwortverhaltens ist an dieser Stelle zum einen kritisch mitzubedenken bzw. sich ins Bewusstsein zu rufen, dass die MSO seit den 2000er Jahren einem wachsenden gesellschaftspolitischen Erwartungsdruck ausgesetzt sind. Zur Veranschaulichung seien hier exemplarisch die bereits erwähnten politischen Aussagen angeführt, dass bürgerschaftliches Engagement auf ,freiwilliger Selbstverpflichtung, öffentlicher Verantwortungsübernahme und Vernetzung“ (BPA 2007: 29) beruhe und dass MSO ,auf allen Ebenen gesellschaftlichen Handelns neue Verantwortung für Integration“ (BPA 2008: 10) zu übernehmen haben (II. 3.2). In diesem Kontext und im Streben nach Anerkennung und Legitimität (II. 4.5) könnten die MSO gewillt sein, den an sie herangetragenen Anforderungen zu entsprechen, d. h. sozial erwünscht zu antworten. Zum anderen können diese externen Ansprüche von den MSO aber auch angenommen bzw. sich zu eigen gemacht worden und - unabhängig von umweltbedingten Einflussnahmen - veritabler Bestandteil der Selbst- oder Leitbilder der MSO sein, die damit auch die organisationale Performanz prägen und mitbestimmen. In der anonym durchgeführten Befragung hätten die MSO jedoch zumindest die Möglichkeit, normativ aufgeladene Leistungs- und Qualitätsanforderungen durch nicht affirmatives Antwortverhalten und mit unerwarteten Gegenbildern zu konterkarieren. Zu guter Letzt ist auch zu bedenken, dass die in den Items mit einzelnen Schlagworten und Phrasen komprimierten Selbstverständnisse unterschiedlich konnotiert bzw. assoziiert worden sein könnten, wenngleich dies durch die Wortwahl (auch in den anderen Sprachen) vermieden werden sollte.

Inwieweit sich die positiven Selbstbilder durch die Übernahme von Tätigkeiten in der Praxis realisieren, wird im Rahmen vorliegender Auswertung separat geprüft (,In-/Kommensurabilitäten“, 7.2.2; 7.2.4).

\section{Das soziale Miteinander in den Organisationen}

Geringe Konfliktdichte und große Bedeutung demokratischer Strukturen sowie gleicher Geschlechterbeteiligung

Die Erhebung des sozialen Miteinanders in einer Organisation zielt auf die Profilierung einer Organisationskultur oder informalen Organisationsstruktur ab. Diese ist relevant für ein intaktes Zusammenwirken, reibungslose Abläufe und die Integration der Mitglieder über geteilte bedeutungsvolle Werte, Überzeugungen oder Rituale, 
die die kollektiven Aktivitäten anleiten und eine Organisationsidentität aufbauen helfen (II. 4.3.4).

Überdeutlich erkennbar sind die hohen, über 95 Prozent liegenden Zustimmungsraten zur Relevanz des internen Zusammenhalts, von demokratischen Strukturen und der Gleichberechtigung von Frauen und Männern sowie einer für 91 Prozent der MSO konstatierten gut funktionierenden Zusammenarbeit zwischen den Hauptamtlichen und Engagierten. Von jeweils circa drei Vierteln der MSO-Vertreter bekräftigt wird zudem die große Bedeutsamkeit von gemeinsamen Ritualen, Festen oder regelmäßigen Zusammenkünften (76\%) sowie die (langjährige) Abwesenheit von Konflikten innerhalb der Organisationen (77\%). Entsprechend wird auch die Existenz von Generationenkonflikten unter den Mitgliedern aufgrund unterschiedlicher Bedürfnisse und Erwartungen mehrheitlich verneint (81\%). Für die religiösen MSO ist eine stärkere Bekräftigung der Wichtigkeit von Ritualen, regelmäßigen Versammlungen (+30 Prozentpunkte) sowie auch eine höhere Zustimmung zur Abwesenheit von Konflikten festzustellen (+12 Prozentpunkte). Allerdings bestätigen religiöse Vereinigungen etwas häufiger das Vorhandensein von Konflikten zwischen jüngeren und älteren Mitgliedern (RMO: $25 \%$ vs. MO: $17 \%$ ).

Ein Austausch mit im Ausland ansässigen Organisationen findet bei 36 Prozent der MSO und unter den nicht-religiösen (40\%) häufiger als unter religiösen Vereinigungen $(28 \%)$ statt. Der Aussage ,Der Glaube an Gott schweißt uns zusammen" wird von 39 Prozent der Vereinigungen und insbesondere von religiösen MSO (89 \%), aber auch von 14 Prozent der sich keiner religiösen Tradition zuordnenden Vereinigungen bekräftigt. Hier zeigt sich abermals, dass der religiöse Glauben auch in,,säkularen“MSO ohne eine offizielle religiöse Traditionszuordnung Relevanz besitzt und dort auch religiös konnotiertes Handeln stattfindet.

\section{- Statistische Tests und Ergebnisdiskussion}

Mit dem $U$-Test kann für die vereinigende Wirkung des geteilten Glaubens sowie die Bedeutung von gemeinsamen Ritualen und regelmäßigen Zusammenkünften für die religiösen Vereinigungen jeweils eine signifikant größere Geltung ermittelt werden. $^{45}$

In Hinblick auf die größere Relevanz von regelmäßigen Zusammenkünften und Ritualen in den religiösen MSO erweist sich also die in der bereits diskutierten Hypothese $\mathrm{H}_{2}$ geltend gemachten größeren Relevanz von regelmäßigen Veranstaltungen als tragfähig, allerdings nicht in Bezug auf effektive Strategien für die Gewinnung

${ }^{45}$ Der Glaube an Gott schweißt uns zusammen $U$-Test: $U=1231 ; z=-10,66 ; p=0,000$; $r=0,7 ; \mathrm{Md}_{\mathrm{S}}=4 ; \mathrm{Md}_{\mathrm{R}}=1$ - Gemeinsame Rituale, Feste, Zusammenkünfte $U$-Test: $U=3129,5 ; z=-6,38 ; p=0,000 ; r=0,42 ; \operatorname{Md}_{\mathrm{S}}=2 ; \mathrm{Md}_{\mathrm{R}}=1$. 
von Freiwilligen $\left(\mathrm{H}_{2}\right)$. Zudem könnte bei dem das Miteinander betreffende Item die „Ausübung von gemeinsamen Ritualen“ für das Ergebnis ausschlaggebend sein. In den Befunden spiegelt sich wider, dass Glaube und Religionspraxis unmittelbar in kollektive Erfahrungen und Emotionen, die die Gemeinschaft gewährleistet, eingebettet sind und dadurch Solidarität und Zusammenhalt ermöglicht werden (Durkheim 1984/[1912]: 580 ff.; Helle 1997: 28).

Die hohen Zustimmungsraten zur Wichtigkeit von demokratischen Strukturen und der gleichberechtigten Geschlechterbeteiligung in der Organisation können einerseits auf ein wesentliches Charakteristikum von freiwilligen Vereinigungen zurückgeführt werden. Denn diese sind von Mitgliedern und Engagierten abhängig und haben deren Interessen adäquat zu berücksichtigen und einzubinden (vgl. hierzu auch Schimank 2000: 310 f.; Borchard/Ceylan 2011). Andererseits könnten sich die Vereinigungen bewusst progressiv und modern präsentieren wollen, um insbesondere verbreitete Ansichten zur Unterdrückung der Frau im Islam und in anderen Kulturen entgegenzuwirken. Insofern sollte die im Rahmen der Befragung vermittelte intakte Organisationkultur auch der Imageverbesserung dienlich und im Rahmen der Öffentlichkeitsarbeit zu betrachten sein.

Des Weiteren scheint die Aufteilung konkreter Tätigkeiten zwischen den Hauptamtlichen und den unentgeltlich arbeitenden Engagierten überwiegend nicht in Konkurrenzsituationen zu münden und ohne größere Probleme zu verlaufen; deren Existenz könnte z. B. aufgrund erforderlicher Aufgabendelegationen an Freiwillige angenommen werden (II. 4.3.4).

Aus religions- und migrationssoziologischer Perspektive ist erwähnenswert, dass in den religiösen MSO zwischen den jungen und den älteren Mitgliedern in einem geringfügigeren, aber nicht signifikanten Ausmaß mehr Unstimmigkeiten registriert werden. Dies ist höchstwahrscheinlich auf veränderte religiöse und soziale Bedürfnislagen der jüngeren, in Deutschland geborenen und herangewachsenen Generationen zurückzuführen (I. 2.2; Nagel 2015a, 2018a, b). Mit der Frequentierung einer religiösen Vereinigung können für die jüngeren Generationen anders gelagerte religiöse Vorstellungen, andere Erwartungshaltungen und Relevanzkriterien einhergehen. Denn religiöse Gehalte werden in einem dynamischen Prozess auf die Gegenwart bezogen, in der Diaspora neu ausgehandelt und aktualisiert (vgl. u. a. Schiffauer 2010; Ceylan 2012a; Antes/Ceylan 2017). Wenn Traditionsbezüge aufbrechen und deren Reproduktion und Institutionalisierung seitens der jüngeren Mitglieder nicht mehr akzeptiert werden, kann die Existenz widerstreitender Auffassungen, Interessen und Wünsche zu Auseinandersetzungen und Verstimmungen zwischen jungen und älteren Mitgliedern führen. Die Daten lassen das Vorhandensein dieser anzunehmenden und im Rahmen anderer Studien empirisch teilweise auch nachgewiesenen Konflikthaftigkeit jedoch nicht in einer erwartbaren Intensität erkennen. 


\subsubsection{Tätigkeitsbereiche und Veränderungen seit der Fluchtdynamik 2015}

\section{Tätigkeitsbereiche der MSO}

(Sozial-)Pädagogische und sozialarbeiterische Dienstleistungen dominieren

Die Ergebnisse zu den Aktivitätsschwerpunkten spiegeln ein breites Spektrum und eine vielfältige Mischung der Aufgaben und Funktionen, die die MSO wahrnehmen. Rund 84 Prozent sind im Feld von Bildung und Erziehung aktiv und jeweils mehr als drei Viertel in der Organisation von Freizeit und geselligen Zusammenkünften (78\%), in der Kinder- und Jugendarbeit (78 \%) sowie im kulturellen Bereich (78\%), beispielsweise im künstlerischen oder musikalischen (Abb. 7.9). Des Weiteren werden konkrete erzieherische und (sozial-)pädagogische Rollen durch Angebote des Deutschlernens (75\%), von Elterngruppen oder Familien(68\%) sowie Mädchen- und Frauenarbeit (61\%) übernommen. In jeweils über 50 Prozent der MSO sind auch die Förderung der Herkunftssprache (59\%), die Selbsthilfe in als belastend wahrgenommenen Lebenssituationen (58\%) sowie die Stadtteilarbeit $(56 \%)$ von Bedeutung.

Der Pflege der Herkunftskultur (49\%) kommt im Vergleich zur Förderung der Herkunftssprache (s. o., $59 \%$ ) - als zwei dezidiert am Geburts- oder Land der Vorfahren orientierte Aktivitäten - eine geringere Bedeutung zu. Die Übernahme von Hilfsdiensten speziell für Geflüchtete (48\%), der Bereich Sport und Bewegung (48\%) sowie Seniorenarbeit (48\%) sind für jeweils weniger als die Hälfte der MSO gleichbedeutende Aufgabengebiete.

Die drei in der Itembatterie berücksichtigten religiösen Aktivitäten werden von weniger als 50 Prozent der MSO ausgeübt. Die Praxis eines interreligiösen Dialogs (47\%) ist geringfügig von größerem Belang als die gleichauf bedeutsamen Bereiche der religiösen Brauchtumspflege (Beten, Feste etc.) (42\%) sowie religiösen Bildung oder Erziehung (42\%). Die drei Tätigkeiten besitzen insbesondere in den religiösen MSO Relevanz (80 \%, 89 \%, $92 \%$ ), aber mit 30, rund 17 und 14 Prozent auch in den nicht-religiösen. 


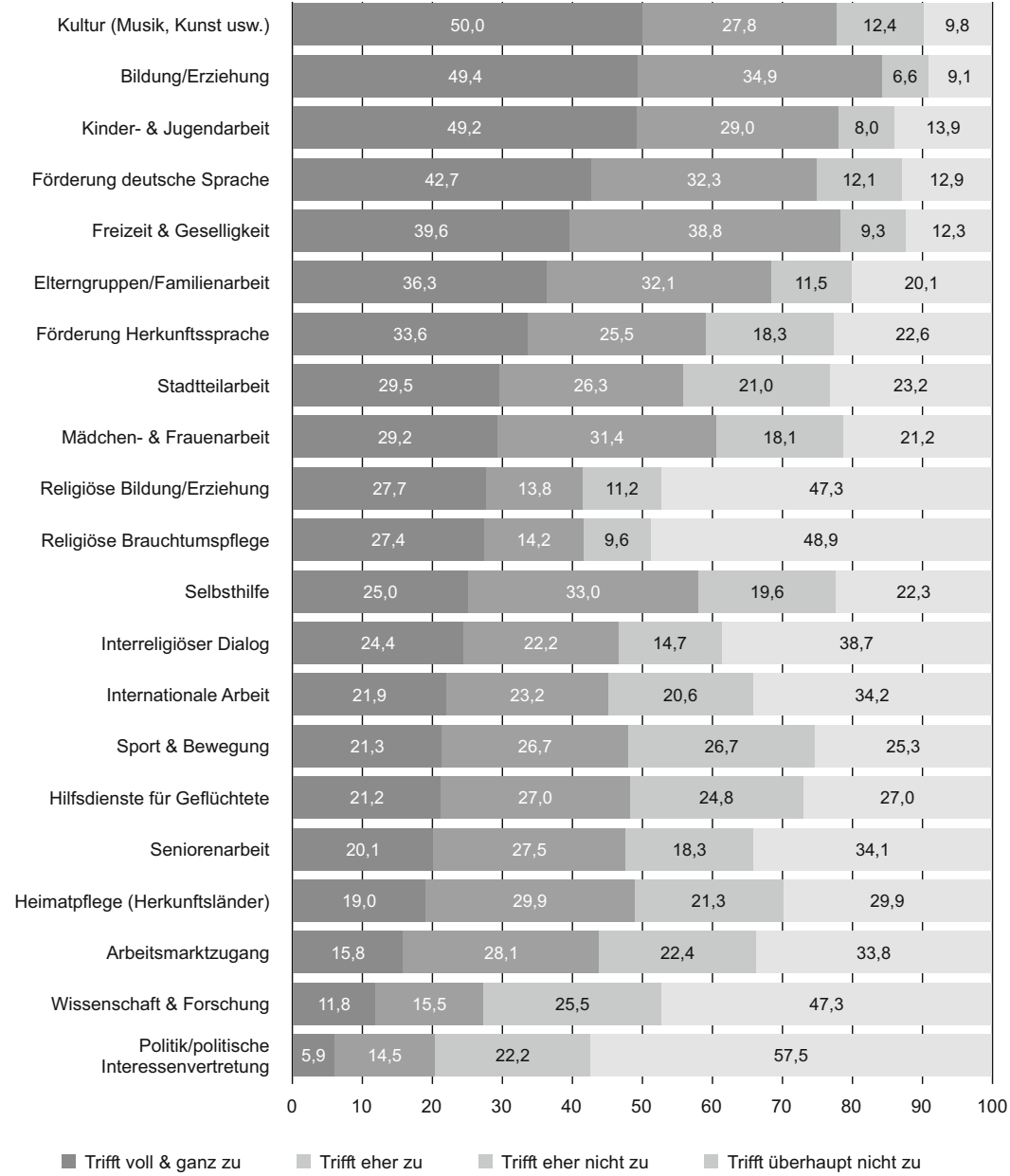

$\mathrm{n}=219$ bis 241

Abb. 7.9 Tätigkeitsbereiche der MSO (in \%) $)^{46}$

${ }^{46}$ Die Ergebnisse der SVR-Studie zu den häufigsten Angebotsbereichen von MSO im Jahr 2019 (maximal 5 Antworten möglich; Friedrichs et al. 2020: 23; I. 2.1) sind mit den hiesigen Befunden aufgrund unterschiedlicher Fragestellungen und Antwortvorgaben nur bedingt vergleichbar: Austausch zwischen Menschen mit und ohne Migrationshintergrund 
Internationale Arbeit (z. B. Entwicklungszusammenarbeit, Einsatz für Menschenrechte) (45\%) wie auch Unterstützungsleistungen beim Zugang zum Arbeitsmarkt (44\%) nehmen in etwa den gleichen Stellenwert ein wie der interreligiöse Dialog (47 \%, s. o.). Dabei ist der Arbeitsmarktzugang für die säkularen MSO ein deutlich wichtigeres Tätigkeitsfeld als für die religiösen (Differenz: 23 Prozentpunkte). Die Schlusslichter der Aktivitätsschwerpunkte stellen Wissenschaft und Forschung (27\%) sowie Politik bzw. politische Interessenvertretung (20\%) dar. Die allgemein abgebildete Präferenz, nicht politisch zu sein oder politisch mitzugestalten, korrespondiert mit der bereits dargelegten wenig ausgeprägten Befürwortung des Ziels, parteipolitische Positionen in Deutschland zu unterstützen (s. o.).

Das breite Spektrum der Aktivitäten und Dienstleistungen zeigt sich besonders durch die große Anzahl der Bereiche, in denen die MSO Tätigkeiten übernehmen. Eine Aufsummierung zeigt, dass über ein Drittel (35\%) schwerpunktmäßig in 10 bis 13 und ein gutes weiteres Viertel (26\%) in 14 Bereichen aktiv ist (Abb. 7.10). Jeweils rund 3 bis 7 Prozent übernehmen ohne nennenswerte Abweichungen auf etwa gleichem Niveau in 1 bis 9 Feldern Aufgaben und Dienstleistungen. Hinsichtlich der Differenzierung zwischen säkularen und religiösen MSO fällt auf, dass letztere häufiger 14 und mehr Sparten nennen (Abb. 7.10; MO: $22 \%$ vs. RMO: $36 \%$ ).

Ungeachtet der tatsächlichen Verteilung der Aktivitäten sprechen die Ergebnisse für eine Selbstdarstellung als besonders aktiv. ${ }^{47} \mathrm{Zu}$ beachten ist jedoch auch, dass sich einzelne Bereiche inhaltlich überschneiden können (z. B. Bildung/Erziehung allgemein und religiöse Bildung/Erziehung).

(45\%), Kinder- und Jugendarbeit (45\%), Bildung (42\%), künstlerisch-kulturelle Aktivitäten (39\%), Unterstützung von Geflüchteten (36\%), Beratung (33\%), Angebote für Frauen (26\%), Eltern-/Familienarbeit (19\%), Pflege der Herkunftskultur(en) (19\%), Antidiskriminierungsarbeit (19\%), politische Interessenvertretung, Religion, Sport (jeweils ca. $15 \%$ ), Pflege/Unterricht der Herkunftssprache (13\%), Entwicklungszusammenarbeit (12\%), Übersetzungen $(11 \%)$, Seniorenarbeit $(10 \%)$, Gesundheit (8\%), Wissenschaft und Forschung (5\%), Arbeitsvermittlung (5\%), Umwelt- und Naturschutz (4\%), Andere (11\%).

${ }^{47}$ Sozial erwünschtes Antwortverhalten (,Tue viel Gutes und zeige es“), um die Zufriedenstellung und Sympathie der Autorin oder der (Fach-)Öffentlichkeit zu generieren, könnten die Beantwortungssituation beeinflusst haben. Dieses ist jedoch gleichermaßen auch bei anderen Fragen (z. B. zu den Zielen und Selbstverständnissen der MSO) nicht auszuschließen. 


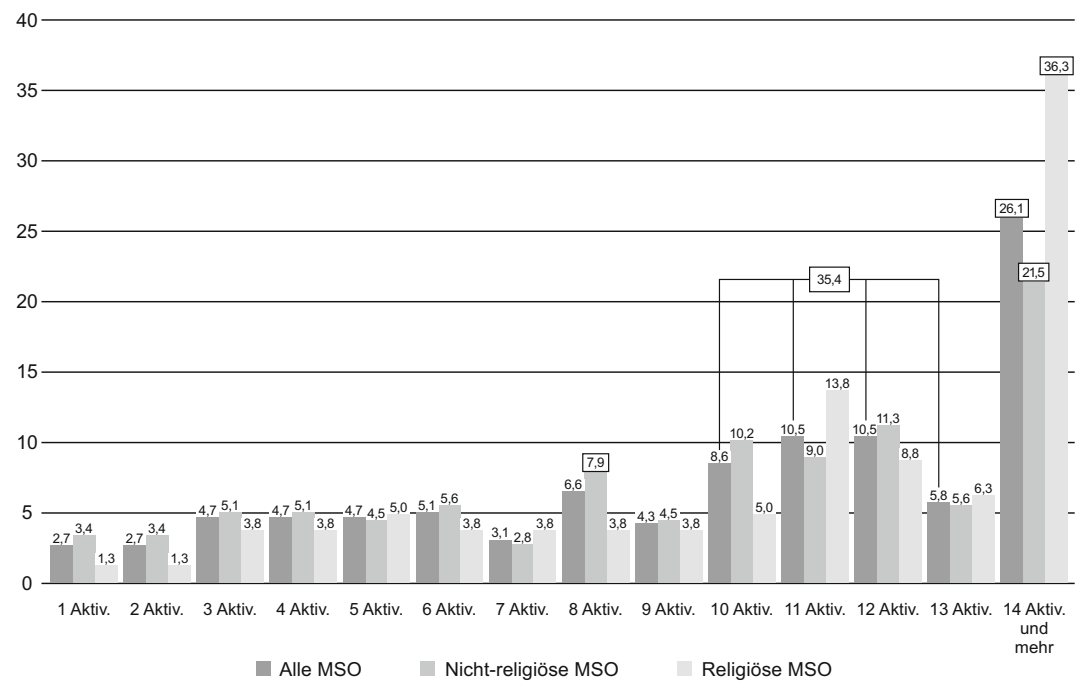

Alle MSO $(n)=257=100 \%$; Nicht-religiöse MSO $\left(n_{S}\right)=177=100 \%$; Religiöse MSO $\left(n_{R}\right)=80=100 \%$.

Aufsummierung der Aktivitäten nach Zustimmung zu den beiden Skalenpunkten „Trifft voll und ganz zu“ und „Trifft eher zu“.

Abb.7.10 Anzahl der Aktivitätsbereiche von (nicht-)religiösen MSO (in \%)

\section{- Statistische Tests und Ergebnisinterpretation}

Religiöse MSO mit Blick auf Anzahl der Tätigkeitsbereiche aktiver

Der $t$-Test zeigt, dass sich die religiösen und nicht-religiösen MSO bezüglich der Anzahl ihrer Tätigkeiten signifikant unterscheiden, wobei die religiösen mit Blick auf die Anzahl der Bereiche aktiver sind. ${ }^{48}$

Die Berechnung des $U$-Tests ermittelt für die religiösen Vereinigungen jeweils eine signifikant größere Bedeutsamkeit der drei Bereiche religiöse Brauchtumspflege, religiöse Bildung/Erziehung sowie interreligiöser Dialog (jeweils große Effekte). Zudem spielt in den religiösen MSO auch die Seniorenarbeit eine gröBere Rolle. In den säkularen MSO besitzen hingegen die drei Tätigkeitsbereiche Kultur allgemein, Bildung/Erziehung sowie Arbeitsmarkt eine jeweils signifikante größere Bedeutung (s. Tab. A 2 im elektronischen Zusatzmaterial).

${ }^{48} t(255)=-2,832 ; p=0,005 ;$ Cohen's $d=0,38$; nicht-religiöse Gruppe: $M=9,77$; $S D=4,61 ; \mathrm{n}_{\mathrm{S}}=177 ;$ religiöse Gruppe: $M=11,56 ; S D=4,85 ; \mathrm{n}_{\mathrm{R}}=80$. 
Bei vielen Gemeinsamkeiten: Religiöses Tätigkeitsprofil in religiösen und heterogenere Aktivitätsfelder in säkularen MSO

Abgesehen von den herausgestellten Unterschieden spiegeln die Testergebnisse in Hinblick auf neun Aktivitäten v. a. auch Gemeinsamkeiten in den Tätigkeitsbereichen der religiösen und nicht-religiösen MSO: Für die Arbeit mit Kindern bzw. Jugendlichen sowie mit Mädchen bzw. Frauen, für die Deutschförderung, Stadtteilarbeit, Flüchtlingshilfe, Internationale Arbeit, die Pflege der Herkunftskultur, Freizeit \& Geselligkeit sowie Sport \& Bewegung sind keine signifikanten Differenzen auszumachen. Unabhängig von kollektiven (nicht-)religiösen Bindungen ist dies ein Beleg für das mögliche vielfältige Nebeneinander zivilgesellschaftlicher Performanzen. Zudem zeigt sich damit auch, dass die beiden konsequent verglichenen MSO-Typen recht konform Tätigkeiten der Sozialen Arbeit (Wohlfahrt) nachgehen, wobei dies mit Blick auf die Seniorenarbeit für die religiösen MSO stärker zutrifft. Dies verweist auf den angesichts des demografischen Wandels wachsenden Stellenwert der Bereitstellung sozialer Dienstleistungen insbesondere auch für ältere Menschen in muslimischen und alevitischen Gemeinden in Deutschland (Halm/Sauer 2015: 105). Im Vergleich dazu fallen die für säkulare MSO signifikant bedeutsameren anderen drei Sparten (Kultur; Bildung/Erziehung; Arbeitsmarkt) hinsichtlich kulturell-sozialer sowie strukturell-integrativer Implikationen als solche durchmischter und uneinheitlicher aus. Inwieweit die Feststellung der Unterschiede und Gemeinsamkeiten mit Blick auf die Grundgesamtheit der MSO in NRW repräsentativ ist, muss aufgrund der defizitären Datenlage (5.3) jedoch offenbleiben.

Die Aktivitäten lassen sich auf Basis der Durchführung einer Explorativen Faktorenanalyse (EFA) bündeln und den folgenden vier Faktoren subsumieren (s. auch Tab. 7.20):

1) (Sozial-)Pädagogische und sozialarbeiterische Dienstleistungen, die Stadtteiloder Gemeinwesenarbeit umfassen;

2) Aktivitäten der Kulturpflege und Freizeit/Geselligkeit;

3) Unterstützungsleistungen, Problembewältigung und Interessenvertretung in der Gemeinschaft sowie in öffentlichen und internationalen Rollen;

4) Religiöse Aktivitäten, die nach innen, an die eigenen Mitglieder, und nach außen gerichtet sind. 
Tab. 7.20 Rotierte Faktorladungsmatrix zu Aktivitätsschwerpunkten

\begin{tabular}{|c|c|c|c|c|}
\hline & \multicolumn{4}{|c|}{ Faktoren } \\
\hline & 1 & 2 & 3 & 4 \\
\hline Elterngruppen/Familienarbeit &, 812 & , 148 & ,130 & 094 \\
\hline Kinder- und Jugendarbeit & ,791 & , 186 &,- 017 & 172 \\
\hline Bildung/Erziehung allgemein &, 756 & ,172 & ,161 &,- 063 \\
\hline Mädchen- und Frauenarbeit & ,709 &,- 036 & ,296 & ,262 \\
\hline Förderung der deutschen Sprache &, 669 & ,386 & ,204 &,- 017 \\
\hline Hilfsdienste speziell für Geflüchtete &, 540 &,- 158 & 465 & 102 \\
\hline Stadtteilarbeit &, 524 & ,130 & ,421 & ,124 \\
\hline Seniorenarbeit* &, 425 & 237 & ,205 & ,335 \\
\hline Heimatpflege (Herkunftsländer) &,- 190 & ,770 & ,175 & ,123 \\
\hline Kultur (Musik, Kunst usw.) & 052 & ,738 &,- 045 &,- 001 \\
\hline Förderung der Herkunftssprache & ,269 & ,670 &,- 039 & 061 \\
\hline Freizeit \& Geselligkeit & ,285 & ,635 &, 023 & , 142 \\
\hline Sport und Bewegung & ,232 & ,584 & ,188 & 020 \\
\hline $\begin{array}{l}\text { Internationale Arbeit (z. B. Entwicklungs- } \\
\text { zusammenarbeit, Menschenrechte) }\end{array}$ & ,067 &, 044 & ,821 & 080 \\
\hline Selbsthilfe & ,336 & 089 & ,626 & ,066 \\
\hline Arbeitsmarktzugang & ,396 & 156 & ,598 &,- 228 \\
\hline Wissenschaft und Forschung & ,231 & ,292 &, 529 & 120 \\
\hline Politik/politische Interessenvertretung &, 012 &,- 039 &, 523 &,- 061 \\
\hline Religiöse Bildung/Erziehung & , 104 &, 019 &,- 048 & ,932 \\
\hline Religiöse Brauchtumspflege (Beten, Feste etc.) &, 029 & ,102 &,- 140 & ,920 \\
\hline Interreligiöser Dialog & ,250 & ,164 & ,298 & ,672 \\
\hline
\end{tabular}

Hauptkomponentenanalyse. Fehlende Werte: paarweiser Fallausschluss. Rotationsmethode: Varimax. KMO-Test: 0,$828 ; \mathrm{n}=219-241$.

* Aufgrund der Ladung unter 0,5 wird das Item ,Seniorenarbeit“ bei der Beschreibung des Faktors nicht explizit berücksichtigt, würde sich jedoch inhaltlich einfügen.

Das auch durch die vier identifizierten Faktoren gespiegelte breite Spektrum der Aktivitäten und Dienstleistungen unterfüttert empirisch die in der theoretischen Fachliteratur thematisierten heterogenen Ausrichtungen sowie die in der Forschung insbesondere für islamische Vereinigungen konstatierte Multifunktionalität, aber auch die beachtenswerte Leitungsfähigkeit der über disparate Ressourcenausstattungen verfügenden MSO.

Deutlich hervor tritt die Übernahme von erzieherischen, (sozial-) pädagogischen und auch sozialarbeiterischen Leistungen (Faktor 1), die auch die Studie des SVR-Forschungsbereichs als einen Tätigkeitsschwerpunkt 
der MSO identifiziert hat (Friedrichs et al. 2020: 26; I. 2.1). Die Angebotserbringung unterstützt die Individuen in ihrer alltäglichen Lebenspraxis bzw. -bewältigung und kann damit auch gesellschaftliche Benachteiligungen auffangen. Stadtteil- oder Gemeinwesenarbeit dient letztlich der Verbesserung des Miteinander-Auskommens und der Lebensbedingungen von unterschiedlichen Bewohnergruppen. Die adäquate und professionelle Ausführung dieser Tätigkeiten setzt spezifische Qualifikationen und Kompetenzen voraus. Inwieweit diese vorhanden sind, lässt sich mit der im Rahmen dieser Arbeit durchgeführten Befragung nicht klären. Gleichermaßen können über eher informell-unverbindliche oder formell-professionelle Ausrichtungen der zivilgesellschaftlichen Aktivitäten keine Aussagen getroffen werden. Unabhängig von einem professionellen Niveau decken diese Arbeiten allerdings Bedarfe und füllen Versorgungslücken.

Bei den dem Faktor 2 subsumierten Tätigkeiten steht hingegen nicht der Anspruch, andere zu unterstützen im Mittelpunkt, sondern die Organisierung von geselligkeits- und herkunftskultur- bzw. traditionsorientierten sowie sportlerischen Interessen, die spontan und zwanglos erfolgen, aber auch leistungsorientiert ausfallen können.

Mit dem Faktor 3 wird das Verlangen nach Unterstützung (aufgrund von Solidarität), Mitgestaltung und Teilhabe in unterschiedlichen Formen und auf verschiedenen gesellschaftspolitischen Ebenen fokussiert. Die Übernahme von (Selbst-)Verantwortung und die Selbstbestimmung bzw. -ermächtigung durch die Befassung mit gruppenspezifischen Problemen wie auch das Ziel der Durchsetzung eigener Interessen implizieren im erhöhten Maße Aspekte von Umgestaltung, Erneuerung und Konflikt. Die organisationalen Handlungen und Maßnahmen können (gleichzeitig) sowohl nach innen als auch nach außen gerichtet $\operatorname{sein}^{49}$ (siehe dazu detailliert 7.2.2). Dies trifft gleichermaßen auf die religiösen Aktivitäten (Faktor 4) zu: Religiöse Bildung/Erziehung und Brauchtumspflege indizieren die Bezogenheit einer religiösen Vereinigung auf die eigene Gruppe, zu der eine Zugehörigkeit bestehen muss. Während der Bereich der religiösen Bildung (Erziehung) die intellektuelle Wissensdimension einer spezifischen Glaubenslehre und ihrer Inhalte umfasst, stellt die Brauchtumspflege die rituelle Komponente kollektiver religiöser Praktiken dar. Der interreligiöse Dialog impliziert über die eigene Gruppe hinausgehende, auf andere religiöse Gemeinschaften ausgerichtete Tätigkeiten. Er ist als eine kommunikative Institution zu betrachten, die auf das

${ }^{49}$ Faktor 3: So lässt sich die Tätigkeit „Internationale Arbeit“ als eine zwischen unterschiedlichen Gruppierungen brückenbildende Tätigkeit kategorisieren (,,bridging“); die „Selbsthilfe“ ist hingegen als eine wesentlich auf die eigene Gruppe fokussierte und nach innen bindende Aktivität einzutufen (,,bonding“). Hier zeigt sich, dass Bridging- und Bonding-Variablen auf denselben Faktor laden können. 
ideologische Selbstverständnis (Wahrheitsanspruch, Heilsvermittlung) und daraus resultierende theologische und alltagspraktische Interaktionsformen mit anderen Religionen bzw. auf die Auseinandersetzung mit deren Glaubenssätzen abhebt.

In der Summe untermauern die im Rahmen der EFA extrahierten Faktoren die Variationsbreite der (gruppierten) Aktivitätsformen, die bei tatsächlicher Durchführung potenziell integrationsförderliche Wirkungen haben können. Denn die selbstorganisierten Zusammenschlüsse dürften den getätigten Angaben zufolge - in unterschiedlichen Intensitätsgraden und Formen - zur Bewältigung verschiedenartig herausforderungsvoller Lebenssituationen von Menschen mit Zuwanderungsgeschichte und deren emotional-psychischen Stabilisierung beitragen. Mit dem Spektrum einhergehend spiegeln die Tätigkeitsbereiche darüber hinaus vier Spannungsachsen, innerhalb derer sie sich bewegen (Tab. 7.21; vgl. auch BMFSFJ 2017: 68-78). Diese Achsen wurden bereits im Kapitel zur Konzeptualisierung von Engagement und Zivilgesellschaft (II. 3.1) thematisiert. ${ }^{50} \mathrm{Sie}$ sind nicht trennscharf voneinander abzugrenzen, sondern können fließend ineinander übergehen und sich miteinander kombinieren. Einerseits sind sie als dezidiert sozialintegrative und teilhabeorientierte Anliegen und entsprechende praxisorientierte Arbeiten einzuordnen (Tab. 7.21; Faktor 1); andererseits bringen sie Bedürfnisse nach Kontaktpflege und Konvivialität zum Ausdruck, die nicht auf nach außen gerichtete Unterstützungsleistungen abheben (Faktor 2). Die Bewahrung von kulturellen und religiösen Werten, Traditionen und Orientierungen (Faktor 2 und 4) steht dem Streben nach gesellschaftspolitischen Veränderungen bzw. Verbesserungen (auch im Sinne von Nachteilsausgleich/Chancengleichheit), dem Wunsch nach Mit- und Umgestaltung, Austausch und (politischer) Fürund Mitsprache in öffentlichen Rollen gegenüber (Faktor 3).

Tab. 7.21 Tätigkeitsbereiche der MSO in vier Spannungsachsen

\begin{tabular}{llll}
\hline Zwischen & Bewahrung kultureller Traditionen & und & Integration/Teilhabe \\
\hline Zwischen & Kontaktpflege/Konvivialität & und & Mit-/Umgestaltung, Mitsprache \\
\hline Zwischen & $\begin{array}{l}\text { Binnenorientierungen der MSO } \\
\text { (,Die MSO nur für und unter sich') }\end{array}$ & und & $\begin{array}{l}\text { Brückenschlägen zu anderen } \\
\text { (,Die MSO auch für andere') }\end{array}$ \\
\hline Zwischen & freiwilliger praktischer Arbeit & und & diskursiver Beteiligung \\
\hline
\end{tabular}

${ }^{50}$ Die in Tab. 7.21 vorgenommene Gegenüberstellung von Spannungsachsen weicht geringfügig von der in Kapitel II. 3.1 erfolgten ab. 
Es zeigt sich, dass praktische Tätigkeiten sowie diskursive Beteiligung und Einmischung einander flankieren können. Gleiches gilt für die Pole der Förderung des sozialen Miteinanders und Zusammenhalts innerhalb einzelner Organisationen (Bindung nach innen) auf der einen und zu anderen Gruppen brückenschlagenden und damit stärker Öffentlichkeitsbezüge implizierenden Engagements auf der anderen Seite (,Brückenbauen“).

Die Faktorisierung der Aktivitäten verweist auf das Zusammenspiel der Tätigkeitsformen sowie das mögliche Zusammengehen dynamischer binnen- und außenorientierter Anliegen. Diesbezügliche Schwerpunkt- und Akzentsetzungen einzelner Organisationen fallen natürlich unterschiedlich aus und werden gesondert und detailliert unter die Lupe genommen (7.2.2).

\section{Religiöses Verhalten auch in ,säkularen“ MSO}

Am Ende dieses Abschnitts zu den Tätigkeitsbereichen ist noch einmal die dominante Ausübung glaubensbasierter Aktivitäten in religiösen MSO zu thematisieren. Sie ist nicht überraschend und lässt sich auf Hauptzwecke der religiösen MSO zurückführen. Dies bedeutet im Umkehrschluss jedoch nicht, dass die sich formal keiner Glaubensgemeinschaft zurechnenden Vereinigungen nicht auch religionsbezogene Aktivitäten ausüben können. Ähnlich wie bei der Mitgliederstruktur oder dem sozialen Miteinander in den MSO (vgl. 7.1.2.1; 7.1.3) wird hier deutlich, dass die per Selbstdefinition vorgenommene Einteilung in nicht-religiöse und religiöse MSO eine hilfreiche Kategorisierung darstellt, die jedoch nicht zur pauschalen Annahme verleiten sollte, dass sich in einer als ,säkular“ klassifizierten Vereinigung konsequent nicht-religiös verhalten wird. Auf dieses Phänomen und mögliche Erklärungen ist im Rahmen eines separaten Analyseschritts zur religiösen Performanz der MSO im Gesamtbild noch zurückzukommen und detaillierter einzugehen ( $\downarrow$ Spotlight: Die religiöse Performanz der MSO).

\section{Aktivitäten in der Flüchtlingshilfe}

Fast die Hälfte der MSO (47 \%; MO: $49 \%$; RMO: $44 \%$ ) übernimmt in der Flüchtlingshilfe eine Vielfalt von bei der Ankunft in Deutschland und für die Integration relevanten Unterstützungsleistungen. Dabei überwiegen Orientierungshilfen hinsichtlich im Aufnahmeland geltender rechtlicher und kultureller Regeln (91\%) sowie im administrativen System durch die Unterstützung bei Behördengängen (88\%) (Abb. 7.11). Dolmetschertätigkeiten (z. B. bei Gesprächen oder Anträgen) $(86 \%)$ sowie Hilfestellungen beim Deutschlernen (76\%) kommen ebenfalls 


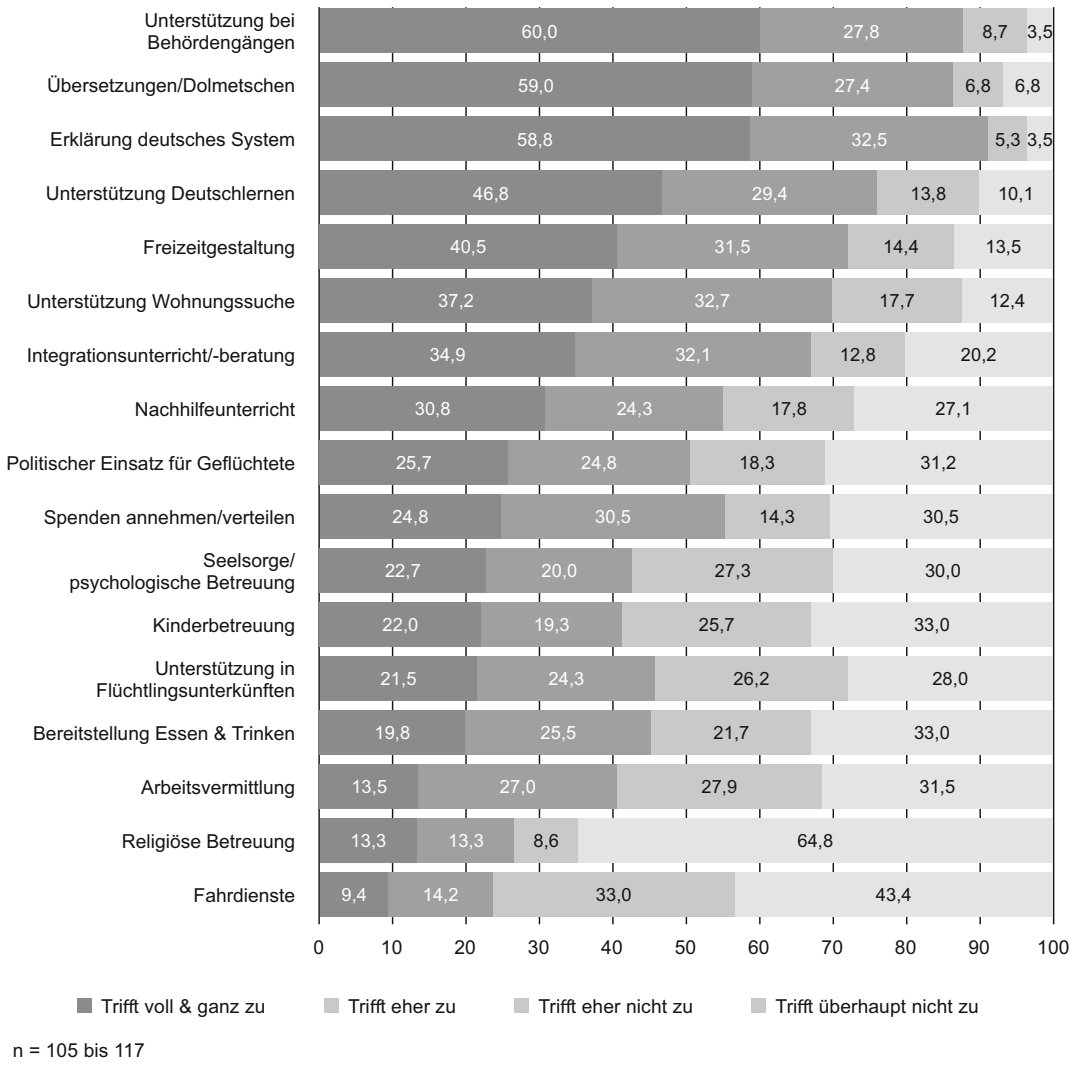

Abb. 7.11 Aktivitäten in der Flüchtlingshilfe (in \%)

eine große Bedeutung zu; sie werden flankiert von weiteren integrativen Maßnahmen wie der Durchführung von Integrationsunterricht bzw. -beratung (67\%), von Nachhilfeunterricht (55\%) oder der Arbeitsvermittlung (41\%). Des Weiteren wird deutlich, dass seitens der MSO in verschiedenen Bereichen ganz praktisch mitangepackt wird, um die Versorgung und Unterbringung von Geflüchteten sicherzustellen und alltägliche Herausforderungen (auch in Flüchtlingsunterkünften) zu meistern. So erfolgt eine Unterstützung bei der Wohnungssuche (70\%), Spenden (z. B. Kleidung) werden angenommen und verteilt (55\%), die Arbeit in Flüchtlingsunterkünften wird unterstützt (46\%), Verpflegung bereitgestellt (45\%), 
Kinderbetreuungen werden übernommen (41\%) und Fahrdienste organisiert (24\%).

Die Tätigkeiten umfassen auch gesellige und emotional stabilisierende Aspekte wie eine gemeinsame Freizeitgestaltung ( $72 \%$ ), eine seelsorgerische bzw. psychologische $(43 \%)$ und in geringerem Ausmaß auch eine religiöse Betreuung $(27 \%)$. Darüber hinaus setzen sich über die Hälfte der in der Flüchtlingshilfe aktiven MSO politisch für die Verbesserung der Situation von Flüchtlingen (51\%) ein. Diese politische Aktivität setzt einen Kontrapunkt zu den ansonsten tendenziell unpolitischen Aktivitäten und Zielen der MSO.

Deutliche Unterschiede in der Aufgabenübernahme zeigen sich hinsichtlich der Unterteilung der MSO in religiöse und nicht-religiöse. Während die säkularen anteilig häufiger Integrationsunterricht bzw. -beratung übernehmen (72\% vs. $55 \%$ ) und bei Behördengängen unterstützen ( $90 \%$ vs. $82 \%$ ), tätigen religiöse Vereinigungen weitaus häufiger eine religiöse Betreuung ( $62 \%$ vs. $7 \%$ ), aber auch eine Versorgung von Geflüchteten mit Lebensmitteln (73\% vs. $34 \%$ ). Zudem finden in den religiösen Vereinigungen häufiger ein Spendenmanagement ( $83 \%$ vs. $45 \%$ ) sowie eine seelsorgerische oder psychologische Betreuung statt (69\% vs. $32 \%$ ). Mit einer Differenz von circa 14 Prozentpunkten übernehmen die religiösen MSO darüber hinaus häufiger Fahrdienste (33\% vs. $20 \%$ ).

\section{- Statistische Tests}

Die Durchführung einer EFA fördert eine Vier-Faktoren-Lösung zutage (Tab. 7.22). Die Faktoren lassen sich wie folgt benennen:

1) Religionsnahe Unterstützungsleistungen, die in religiöse Gemeindestrukturen eingebettet sind;

2) Freizeitbeschäftigung und haushaltsnahe, niedrigschwellige Unterstützung;

3) Hilfeleistungen, die spezifische Qualifikationen voraussetzen und Aspekte der strukturellen Integration betreffen;

4) Unterstützungsleistungen, die die strukturelle, kulturelle und soziale Integration betreffen und die ggf. politisch motiviert sind.

Gemeindenahe Unterstützungsleistungen in religiösen Vereinigungen versus höhere Affinität zu administrativ-rechtlichen Angelegenheiten in säkularen MSO

Ergebnisse des $U$-Tests lassen die zwei MSO-Typen deutlich hervortreten. Sie zeigen, dass neben der religiösen Betreuung in den religiösen MSO auch die vier Tätigkeitsbereiche Verpflegung, Spendenverwaltung, Seelsorge und Fahrdienste von größerer Bedeutung sind (s. Tab. A 3 im elektronischen Zusatzmaterial). Es 
Tab. 7.22 Rotierte Faktorladungsmatrix zu Aktivitäten in der Flüchtlingshilfe

\begin{tabular}{|c|c|c|c|c|}
\hline & \multicolumn{4}{|c|}{ Faktoren } \\
\hline & 1 & 2 & 3 & 4 \\
\hline Religiöse Betreuung & 824 &,- 015 &,- 015 &,- 115 \\
\hline Bereitstellung von Essen und Trinken &, 770 & ,200 &, 069 & 140 \\
\hline Fahrdienste & ,727 & ,069 & 071 & ,282 \\
\hline Seelsorge/psychologische Betreuung & ,667 & ,238 & , 149 & 097 \\
\hline Spenden annehmen/verteilen (z. B. Kleidung) & ,626 & 430 & , 166 & 178 \\
\hline Gemeinsame Freizeitgestaltung & ,125 & ,761 &, 041 & ,224 \\
\hline Unterstützung beim Deutschlernen & 259 &, 694 &, 472 &,- 085 \\
\hline Kinderbetreuung & ,250 & ,673 &, 119 & ,252 \\
\hline Unterstützung bei Behördengängen & 001 & 040 & ,903 &, 132 \\
\hline $\begin{array}{l}\text { Übersetzungen/Dolmetschen } \\
\text { (z. B. bei Gesprächen oder Anträgen) }\end{array}$ & 153 & 103 & ,857 & ,141 \\
\hline $\begin{array}{l}\text { Erklärung des deutschen Systems } \\
\text { (Gesetze, Kultur, Werte) }\end{array}$ & ,042 &, 254 & ,674 & ,339 \\
\hline Unterstützung bei der Wohnungssuche & 329 &, 550 &, 561 & ,136 \\
\hline Unterstützung der Arbeit in Flüchtlingsunterkünften & 090 &,- 020 &, 130 & ,816 \\
\hline $\begin{array}{l}\text { Politischer Einsatz für die Verbesserung der Situation von } \\
\text { Flüchtlingen }\end{array}$ & ,123 & 122 & ,266 & ,690 \\
\hline Integrationsunterricht/-beratung &,- 113 & ,496 &, 085 & ,619 \\
\hline Arbeitsvermittlung & ,216 & ,252 &, 088 & ,596 \\
\hline Nachhilfeunterricht & 185 & ,521 & , 127 & ,571 \\
\hline
\end{tabular}

Hauptkomponentenanalyse. Fehlende Werte: paarweiser Fallausschluss. Rotationsmethode: Varimax. KMO-Test: 0,$838 ; \mathrm{n}=105-117$.

handelt sich damit um genau die Aktivitäten, die auch auf den Faktor 1 laden. In den säkularen Vereinigungen werden die geflüchteten Menschen hingegen häufiger bei Behördengängen unterstützt.

Diese Befunde sprechen dafür, dass insbesondere religiöse Vereinigungen als Orte und Anlaufstellen fungieren, an denen eine adäquate religiöse bzw. seelsorgerische Betreuung von religiös orientierten geflüchteten Menschen stattfinden kann, die diese MSO höchstwahrscheinlich gezielt aufsuchen; zugleich kann über einen geteilten Glauben in prädestinierter Weise ein Zugang zu ihnen und ihren Schicksalen gefunden und eine Vertrauensbasis aufgebaut werden. Auch kann ein in den religiösen MSO vorhandenes größeres Verantwortungs- bzw. Pflichtgefühl für die Geflüchteten zu sorgen und Notwendiges zu tun, um Schaden abzuwenden, relevant sein. Zudem erscheint die Annahme plausibel, dass die häufigere Übernahme von primär nicht religiös ausgerichteten Tätigkeiten - wie die Verpflegung von Geflüchteten, die Verwaltung von Spenden sowie Fahrdienste - aufgrund 
regelmäßiger Interaktionen, gut organisierter und intakter Gemeindeinfra- und Beziehungsstrukturen bzw. an sie gekoppelt und im Rahmen bereits existenter Angebote und Dienstleistungen erfolgt.

Die in größerem Umfang durch nicht-religiöse Vereinigungen erfolgende Unterstützung bei Behördengängen verweist auf die Komponente struktureller Integrationsmaßnahmen, die mit einer höheren Affinität zu administrativen und juristischen Angelegenheiten und einem besseren Draht der säkularen MSO zu amtlichen Dienststellen und Verwaltungsorganen zu erklären sein könnte. Diese Interpretation erscheint auch vor dem Hintergrund der im weiteren Textverlauf dargelegten Ergebnisse zu intensiveren Beziehungen zwischen säkularen und städtischen bzw. staatlichen Akteuren plausibel zu sein: Wertschätzende Zusammenarbeit wie auch finanzielle Förderungen in der Flüchtlingshilfe - und über diese hinausgehend - sind insbesondere für nicht-religiöse Vereinigungen relevant (s. dazu im Folgenden auch 7.1.6).

In der Summe lässt sich somit annehmen, dass die vorhandenen Infrastrukturen, geteilte Herkunftsbezüge, gemeinsame Sprachen, Glaubenszugehörigkeiten, Erfahrungen und Bedürfnisse einen guten Ausgangspunkt und Nährboden für Solidarität und den Aufbau von Vertrauen zu vulnerablen Gruppen geflüchteter Menschen darstellen können (vgl. auch Nagel/El-Menouar 2017: 27, 45; Friedrichs et al. 2020: 31). Diese günstigen Bedingungen werden in unterschiedlichen Arbeitsbereichen und Politikfeldern unter den Aspekt des ,Zugangs zur Zielgruppe" gefasst, über den MSO in herausragender Weise verfügen, weshalb ihnen als Kooperationspartner und integrationspolitische Akteure bedeutende Rollen zugeschrieben werden (BT 2016a; LT NRW 2018).

Grundsätzlich ist hinsichtlich der Angabe von in der Flüchtlingshilfe seitens der MSO übernommenen Tätigkeiten zu bedenken, dass diese Momentaufnahmen darstellen und konjunkturellen Schwankungen unterliegen dürften, da Unterstützungsbedarfe je nach dem Ausmaß von Fluchtzuwanderung und resultierenden Fluktuationen in der Frequentierung der MSO durch geflüchtete Menschen unterschiedlich stark benötigt und angefragt werden.

\section{Veränderungen für die MSO seit der Fluchtdynamik 2015}

Die 120 in der Flüchtlingshilfe aktiven MSO sollten in Bezug auf seit der Fluchtdynamik 2015 für sie zu registrierende Veränderungen zu vier Antwortvorgaben Einschätzungen vornehmen. Für 63 Prozent der MSO trifft die Aussage, dass ihre Organisation zur Anlaufstelle für Geflüchtete geworden ist, ,,voll und ganz“ bzw. „,eher zu“. Diesbezüglich fallen die Zustimmungsraten für religiöse und säkulare 
MSO identisch aus (MO: $63 \%$; RMO: $63 \%$ ). Die Zusammenarbeit mit städtischen Ämtern und Behörden hat sich für 65 Prozent der befragten Organisationen intensiviert (Abb. 7.12). Das Statement, dass ihre Arbeit seitens der Stadt und Politik eine höhere Aufmerksamkeit und Wertschätzung erfährt, wird von 53 Prozent bestätigt. Von politischen Fördermaßnahmen profitieren 37 Prozent der in der Flüchtlingshilfe aktiven MSO.

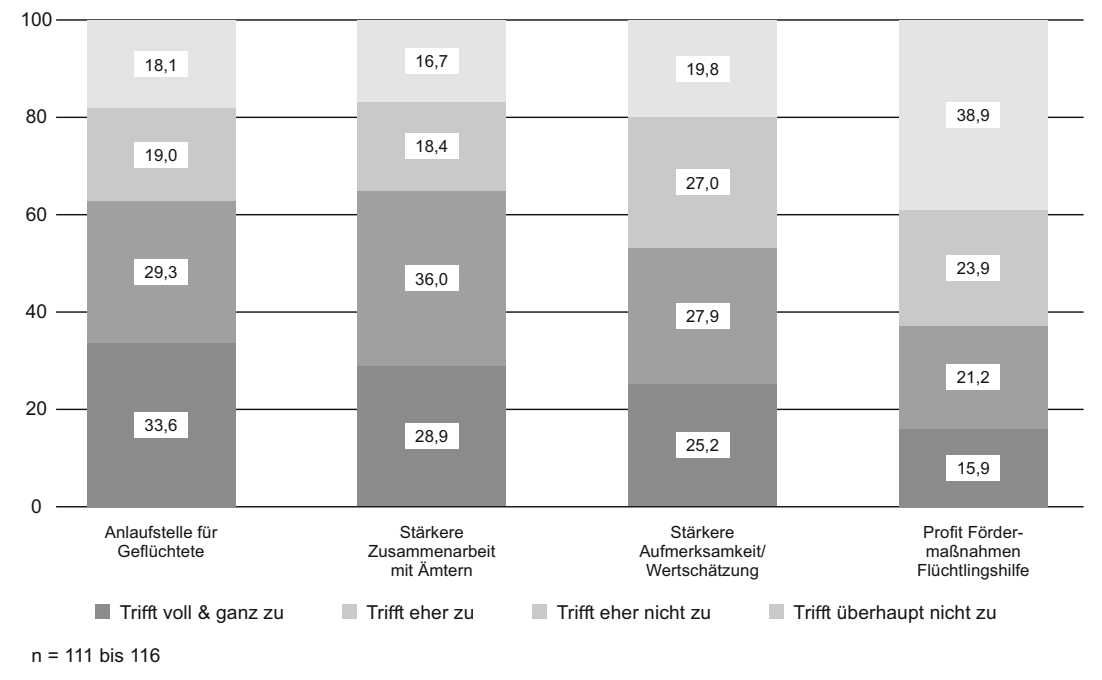

Abb.7.12 Veränderungen für MSO seit der Fluchtdynamik 2015 (in \%)

Entlang der Differenzierung zwischen religiösen und nicht-religiösen MSO zeigen sich Unterschiede, die eine ungleiche Behandlung andeuten. Religiöse MSO nehmen seitens der Stadt und Politik deutlich weniger Aufmerksamkeit und Wertschätzung wahr (RMO: 39 \% vs. MO: 59 \%); für sie hat sich auch die Zusammenarbeit mit städtischen Ämtern und Behörden in einem geringeren Ausmaß intensiviert (50\% vs. $71 \%$ ). Gleiches gilt für politische Fördermaßnahmen in der Flüchtlingshilfe, von denen die religiösen MSO seltener profitiert haben als die nicht-religiösen ( $25 \%$ vs. $42 \%$ ). 


\section{- Statistische Tests und Ergebnisinterpretation}

Flüchtlingshilfe: Größere städtische und politische Anerkennung von säkularen MSO

Ergebnisse statistischer Berechnungen zeigen für die beiden Merkmale der Wahrnehmung von mehr Aufmerksamkeit und Wertschätzung sowie der Zusammenarbeit mit städtischen Einrichtungen jeweils stärkere Ausprägungen bei den nichtreligiösen MSO.${ }^{51}$ Die Ergebnisse korrespondieren mit der obigen Feststellung einer für religiöse MSO niedriger ausfallenden finanziellen Förderung durch die Städte (7.1.2.2), die sich im Rahmen der Flüchtlingshilfe jedoch nicht im signifikanten Ausmaß zeigt.

Für die Deutung der Ergebnisse hilfreich ist eine Inblicknahme der auf kommunaler Ebene in NRW bestehenden Kriterien für die Förderung von und Zusammenarbeit mit MSO: Ein integrativer Fokus ihrer Aktivitäten sowie die Grundgesetzkonformität bzw. Akzeptanz der freiheitlich-demokratischen Verfassung stellen zwei zentrale Bedingungen dar. Kooperiert wird mit integrativ arbeitenden und an der Gestaltung des lokalen Zusammenlebens interessierten MSO, die über gute Zugänge zu Zielgruppen verfügen, die über die eigene Community hinausgehen. Wenn neben nicht zuwendungsfähigen religiösen oder kulturellen Aktivitäten auch Integrationsarbeit geleistet wird, können auch religiöse MSO unterstützt werden (Klie 2020 ${ }^{52}$ ). Bei letzteren wird seit 2015 ein erhöhter Zulauf von Geflüchteten registriert, was zu einer stärkeren Projektförderung von Moscheegemeinden in der Flüchtlingshilfe geführt hat. Allerdings werden zugleich auch Rückzugstendenzen einiger MSO beobachtet und in den türkischen Moscheegemeinden wird eine starke (politische) Beeinflussung der Einstellungen der Mitglieder durch das Heimatland registriert (ebd.; Güvercin 2019). Dies führt teilweise zu einer unsicheren, zwiespältigen und kritischen städtischen Haltung insbesondere gegenüber DİTIB-Gemeinden als ehemals hochgeschätzte Kooperationspartner und auch zu Kontaktabbrüchen. Diese Verunsicherung angesichts dynamischer politischerEntwicklungen in der Türkei, der resultierenden Verschlechterung der Beziehungen zu Deutschland sowie der Beobachtung einer starken, nicht geduldeten Einflussnahme können daher durchaus als eine mögliche Ursache für eine geringer ausgeprägte städtische Zusammenarbeit mit und Wertschätzung von islamischen MSO betrachtet werden. Diese kann dann auch

${ }^{51}$ Aufmerksamkeit und Wertschätzung $U$-Test: $U=901,5 ; z=-2,57 ; p=0,01 ; r=0,24$; $\left.\mathrm{Md}_{\mathrm{S}}=2 ; \mathrm{Md}_{\mathrm{R}}=3\right)$ - Intensivierung Zusammenarbeit mit städtischen Einrichtungen $U$-Test $U=916 ; z=-2,6 ; p=0,009 ; r=0,24 ; \mathrm{Md}_{\mathrm{S}}=2 ; \mathrm{Md}_{\mathrm{R}}=2,5$.

${ }^{52}$ Die Darstellungen zu den Förderkriterien basieren auf einem Screening von Leitlinien der MSO-Förderung in NRW-Kommunen sowie fünf im Sommer 2017 seitens der Autorin dieser Arbeit in zwei Kommunen in NRW durchgeführten leitfadengestützten Experteninterviews. 
politische Würdigungen der MSO - und insbesondere der islamischen Verbände - als unverzichtbare „Brückenbauer“, ,Kulturdolmetscher“ und „Vorbilder für Flüchtlinge“konterkarieren (u.a.BT 2016a); aufgrund angespannter(politischer) Verhältnisse realisiert sich die proklamierte Anerkennung de facto nicht konsequent.

Die stärkere Beteiligung von und Zusammenarbeit mit säkularen MSO seitens politischer Akteure und Einrichtungen sind somit einerseits auf förderpolitische Richtlinien zurückzuführen, die letztlich die nicht-religiösen MSO begünstigen. Andererseits ist jedoch zu erwägen, dass die säkularen MSO eine größere Nähe zu integrationspolitischen Aktivitäten bzw. eine dezidierte Ausrichtung auf solche aufweisen dürften. Ihre Tätigkeitsbereiche und Interessenlagen sollten sich daher stärker mit denjenigen von anderen in der Integrationsarbeit beteiligten Akteuren überschneiden. Die unterschiedliche Ausgangslage für säkulare und religiöse MSO muss folglich nicht zwangsläufig auf Reglementierungsbedarfe und intendierte Sanktionsbzw. Exklusionsmechanismen zurückzuführen sein, sondern sie kann sich ggf. auch einfach ergeben und entwickelt haben, da nicht-religiöse MSO und städtische Einrichtungen in gleichen Feldern arbeiten, mehr Berührungspunkte aufweisen und sozusagen ,an einem Strang ziehen“.

Davon unabhängig kann auch das im übernächsten Abschnitt (7.1.6) thematisierte insgesamt weniger intensive Vernetzungsverhalten der religiösen MSO eine Begründungsfolie für die erkennbaren Unterschiede darstellen.

\section{- Spotlight: Die religiöse Performanz der MSO}

Für eine präzisere Beschreibung der religiösen Performanz der MSO wurden acht im Fragebogen enthaltene religionsbezogene Items (Subdimensionen) zusammen betrachtet und entsprechend zahlenmäßig auftretender Kombinationen kategorisiert (Tab. 7.23). Bei den fokussierten Subdimensionen handelt es sich um das Organisationsziel der „gemeinschaftlichen Glaubenspraxis“, das organisationale Selbstverständnis eine „Religionsgemeinschaft“ zu sein, die Aussage, dass der „Glaube an Gott die Mitglieder zusammenschweißt“, sowie die drei Aktivitäten „Religiöse Bildung/Erziehung“, ,,Religiöse Brauchtumspflege“ und „Interreligiöser Dialog“. Des Weiteren wurden die beiden für die Engagierten abgefragten Motive „Anderen den Glauben nahebringen“ und „Religiöse Werte und Gebote umsetzen“ einbezogen. Angemerkt sei, dass die Motive nur dann als religiöse Aspekte mitberücksichtigt werden konnten, wenn die MSO über Engagierte verfügen (was lediglich bei $6 \%$ der MSO nicht der Fall ist). Bei der Analyse wurde die Tätigkeit der religiösen Betreuung von Geflüchteten ausgeklammert, da dieses Item nur dann zu beantworten war, wenn die vorgeschaltete Filterfrage nach Tätigkeitsübernahmen in der Flüchtlingshilfe bejaht wurde. 
Tab. 7.23 Religiöse Performanzen im Gesamtbild: Kategorisierung religionsbezogener Items nach Antwortverhalten (in absoluten und relativen Häufigkeiten)

\begin{tabular}{lrrrc}
\hline $\begin{array}{l}\text { Anzahl religiöser Items } \\
\text { (min.: 0 / max.: 8) }\end{array}$ & Häufigkeit & Prozent & $\begin{array}{c}\text { Nicht-religiöse } \\
\text { MSO }\end{array}$ & $\begin{array}{c}\text { Religiöse } \\
\text { MSO }\end{array}$ \\
\hline 1 & 31 & 12,9 & 31 & 0 \\
\hline 2 bis 3 & 25 & 10,5 & 25 & 0 \\
\hline 4 bis 5 & 19 & 7,9 & 5 & 14 \\
\hline 6 bis 7 & 42 & 17,5 & 4 & 38 \\
\hline $8=$ Komplett religiös & 29 & 12,1 & 2 & 27 \\
\hline $0=$ Komplett nicht-religiös & 78 & 32,6 & 78 & 0 \\
\hline $0=$ Nicht-religiös (1 bis 5 fehlende Ang.) & 15 & 6,2 & 15 & 0 \\
\hline Gesamt & 239 & 100,0 & 160 & 79 \\
\hline Fehlende Angaben (komplett) & 18 & & & \\
\hline
\end{tabular}

$\mathrm{n}=239$; nicht-religiöse MSO $\left(\mathrm{n}_{\mathrm{S}}\right)=160$; Religiöse $\operatorname{MSO}\left(\mathrm{n}_{\mathrm{R}}\right)=79$.

Um komplett bzw. mehr oder weniger religiöse oder nicht-religiöse Performanzen der MSO feststellen zu können, wurden die jeweils vorgegebenen vier Antwortmöglichkeiten („,Trifft voll und ganz“...,,Trifft überhaupt nicht zu“) in zwei Kategorien zusammengefasst, die für die Auswertung wiederum als Bejahung und Verneinung klassifiziert wurden. ${ }^{53}$ In Bezug auf die analyserelevanten Items waren für $18 \mathrm{MSO}$ komplett fehlende Angaben auszumachen (Tab. 7.23). Fälle mit einzelnen fehlenden Angaben wurden bei der Analyse nicht berïcksichtigt; wenn sie aber in Kombination mit der Verneinung einer Mehrzahl der religiösen Subdimensionen auftraten, wurden sie explizit als solche ausgewiesen (Tab. 7.23). ${ }^{54}$

Anhand dieses separaten Analyseschritts kann aufgezeigt werden, dass rund 33 Prozent der MSO - und zwar ausschließlich nicht-religiöse - komplett nicht-religiös agieren. Weitere 13 Prozent der MSO (ebenfalls lediglich nicht-religiöse) bejahen ein einziges religiöses Item (Tab. 7.23), wobei es sich zumeist um die Ausübung einer religiösen Aktivität (10\%) handelt, und zwar überwiegend um den interreligiösen Dialog 55 .

53 „Trifft voll \& ganz zu/Trifft eher zu“ = Bejahung; ,,Trifft eher nicht zu/Trifft überhaupt nicht $\mathrm{zu}^{\prime \prime}=$ Verneinung.

${ }^{54}$ Die fehlenden Werte wurden in diesen Fällen gesondert ausgewiesen, da ihre Interpretation als Nicht-Zustimmungen und eine entsprechende Feststellung eines ,komplett religiösen Verhaltens" für die betreffenden MSO als ein zu grobes Vorgehen mit der Gefahr falscher Schlussfolgerungen betrachtet wurde.

${ }^{55}$ Von 8 MSO (3\%), die eine religiöse Subdimension bejaht haben, wurden jeweils dreimal die beiden Motive „Anderen den Glauben nahebringen“ sowie „Religiöse Werte und Gebote umsetzen“ als zutreffend markiert; in zwei weiteren Fällen wurde ,Der Glaube an Gott 
Ein komplett religiöses Profil weisen circa 12 Prozent der MSO auf. Unter diesen befinden sich zwei säkulare Vereinigungen. Rund 18 Prozent haben 6 bis 7 der religiösen Subdimensionen bejaht, darunter sind 4 der nicht-religiösen Organisationen. Bei 8 Prozentpunkten von diesen 18 Prozent wurden ein oder zwei der religiösen Motive verneint oder zu diesen keine Angabe gemacht. Unter den anderen 10 Prozentpunkten, für die 6 bis 7 der religiösen Aspekte zutreffen, wurden zumeist (in Kombination) das Selbstverständnis als Religionsgemeinschaft und die Aussage, dass der Gottesglaube die Mitglieder zusammenschweißt, verneint oder einem der religiösen Motive der Engagierten oder der Ausübung von einer der drei religiösen Aktivitäten nicht zugestimmt.

Für 11 Prozent der MSO - ausschließlich nicht-religiöse - wurden 2 oder 3 der religiösen Aspekte geltend gemacht. Die einzelnen affirmierten Punkte betreffen hier eine oder zwei religiöse Aktivität(en), das Organisationsziel der gemeinsamen Glaubenspraxis und das durch den Gottesglauben gefestigte Miteinander; in einigen Fällen wird auch ein Motiv bestätigt.

Für weitere 8 Prozent der MSO erweisen sich 4 oder 5 der religiösen Subdimensionen als zutreffend; diese MSO rechnen sich mit einerZahl von 14 der hier mehrheitlich einer religiösen Lehre zu (18 \% der religiösen MSO). Bei den bestärkten religiösen Items handelt es sich um die soeben genannten (Aktivitäten; Ziel Glaubenspraxis; durch Glaube gefestigtes Miteinander) und zusätzlich um das Selbstverständnis als Religionsgemeinschaft, ein zweites Motiv oder eine weitere Aktivität.

\section{- Ergebnisdiskussion}

Religiöses Verhalten auch in ,säkularen“ MSO

Die Auswertungsergebnisse zeigen insgesamt, dass der auf der Strukturebene der MSO angesiedelte dichotomische Analysezugriff religiös versus säkular, der über die eigene (Nicht-)Zuordnung der MSO zu einer bestimmten religiösen Tradition oder Lehre erfolgte, ein belastbares Unterscheidungskriterium ist: Anhand der separaten Analyse lässt sich eindeutig erkennen, dass sich religiöse Vereinigungen dominant religiös und religiöser gerieren als die säkularen. Das ist sinnfällig und logisch eingängig. Die Ergebnisse zeigen, dass eine konstatierte Zugehörigkeit zu einer Konfession auf der Organisationsebene ein guter Indikator für organisationale religiöse Performanz darstellt und dementsprechend eine Nicht-Zuordnung als ein guter Anhaltspunkt für nicht-religiöses Verhalten in säkularen Vereinigungen fungieren kann - allerdings nicht in einem hinreichenden Maße.

schweißt uns zusammen“ sowie das Organisationsziel „Den religiösen Glauben gemeinsam praktizieren" angegeben. 
Wie auch bereits im Abschnitt zu den allgemeinen Tätigkeitsbereichen der MSO thematisiert wurde (7.1.4), können religiöse Überzeugungen und Praktiken auch in säkularen Vereinigungen von Bedeutung sein. Anhand der Befunde wird deutlich, dass die Ausübung religiöser Aktivitäten nicht zwangsläufig religiöser Dispositionen der Engagierten oder der Wahrnehmung eines auf einem gemeinsamen Gottesglauben basierenden Zusammenhalts bedarf. Zudem ist denkbar und für drei Fälle zutreffend (vgl. Fußnote 55), dass religiöse Motive der Engagierten bejaht werden, in den betreffenden nicht-religiösen MSO allerdings keinerlei weitere religiöse Subdimensionen relevant sind. Grundsätzlich lässt sich anhand der Ergebnisse erkennen, dass sich religiöse Subdimensionen in unterschiedlichen Ausmaßen und Intensitätsgraden miteinander verquicken oder eben nicht und das - insbesondere mit Blick auf religiöse Vereinigungen - daher zwar von wechselseitiger Stimulierung, aber nicht grundsätzlich von durchgängiger Relevanz oder konsequenten Wenn-Dann-Verkettungen der religiösen Subdimensionen auszugehen ist.

In den als ,nicht-religiös“ etikettierten MSO kann also mehr Religion vertreten sein als qua Labeling induziert. Wenngleich dieses Phänomen zunächst kontraintuitiv zu sein scheint, ist es gleichwohl gut nachvollziehbar, denn religiös konnotiertes menschliches Verhalten ist nicht bedingt durch ein offizielles Bekenntnis zu einer Religion auf der strukturellen Ebene einer Organisation. ${ }^{56}$ Zudem ist im Zusammenhang mit der Ausübung religionsbezogener Tätigkeiten nicht zwangsläufig von einer auf der Individualebene stark ausgeprägten Religiosität auszugehen, allerdings von einer gewissen Wertschätzung von und Affinität zu religiösen und theologischen Themen und Praktiken. Die Durchführung von religiöser Bildung/Erziehung bzw. des interreligiösen Dialogs setzt zudem Fachwissen und pädagogische Kompetenzen voraus. Darüber hinaus sollte sich in der organisationalen Praxis einer MSO, die sich keiner bestimmten Religion zuordnet, als relevant erweisen, dass sich die Mitglieder bzw. Teilgruppen von Mitgliedern im Alltag nicht ohne Weiteres von ihrer Religionszugehörigkeit trennen lassen bzw. diese Eigenschaften nicht einfach ignoriert werden können. Insofern könnte davon ausgegangen werden, dass religiöse Bedürfnisbefriedigungen - auch ohne eine auf der Mesoebene offiziell

\footnotetext{
${ }^{56}$ Das Argument, dass es Organisationen gibt, die sich auf der strukturellen Ebene keiner religiösen Tradition zuordnen, die aber dennoch eine starke religiöse Performanz aufweisen, ist an dieser Stelle noch einmal kritisch zu akzentuieren. Denn die Tatsache, dass einzelne nicht-religiöse MSO (fast) alle abgefragten religiösen Items bejaht haben, sollte nicht überbewertet werden. So ist nicht auszuschließen, dass sich die Funktionsträger, die die Frage „Ordnet sich Ihre Organisation einer bestimmten religiösen Tradition oder Lehre zu?“ für die betreffenden Organisationen online beantwortet haben, schlichtweg verklickt oder dass sie die Formulierung missverstanden oder als für ihre Organisation als zu spezifisch und formal(isierend) eingestuft haben.
} 
organisierte und propagierte Religionstradition - im Kontext eines herkunftsübergreifend und interkulturell ausgerichteten Vereins eine Rolle spielen. Insofern sind religiöse Praxis und religiöse Akkomodation (engl.: ,religious accomodation“) ohne eine offizielle religiöse Affiliation auf Organisationsebene durchaus nachvollziehbare Phänomene. Herauszustreichen ist an dieser Stelle, dass ,säkular“ nicht im Sinne von ,anti-religiös“ zu verstehen ist. Als erkenntniserweiternd sollte sich erweisen, wenn den hier im Zusammenhang mit empirischen Befunden quantitativer Forschung dargelegten Erörterungen in einem qualitativen Befragungsdesign tiefergehender nachgegangen wird.

Des Weiteren erscheint es plausibel, dass eine Nicht-Zuordnung einer MSO zu einer religiösen Tradition mit Angebotserbringungen im religiösen Bereich einhergehen kann, wenn man sich die konkreten Situationen in den nordrheinwestfälischen Kommunen vor Augen führt: Die Pluralität von Menschen aus verschiedensten Herkunftsländern und Kulturen bzw. mit unterschiedlichen Glaubenszugehörigkeiten ist ein Faktum, das das Zusammenleben in den Kommunen stark herausfordert und das die integrationspolitischen Handlungsfelder sowie die in diesen bedeutsamen Rollen der über gute Zugänge zu schwer erreichbaren Zielgruppen verfügenden MSO maßgeblich prägt. Insbesondere in einzelnen Stadtteilen und Quartieren ist die Förderung von interkulturellem bzw. interreligiösem Austausch und von Begegnungen, um das Zusammenleben aktiv zu gestalten, um Vorurteile und Missverständnisse abzubauen und Segregation zu vermeiden, zu einem wichtigen integrationspolitischen Handlungsfeld geworden. In der Sozialarbeit ist folglich insbesondere auch die Bearbeitung des Konfliktfelds Religion für die Prävention von religiös begründetem Extremismus ein zentrales Unterfangen. In diesem Kontext zeigt sich in der kommunalpolitischen Praxis ganz konkret, dass auch MSO, die sich dezidiert als säkular bzw. nicht explizit als religiös verstehen, die Förderung von Austausch und Begegnung auf ihre Agenden geschrieben haben und in diesem Rahmen auch religionsbezogenen Aktivitäten nachgehen. $\mathrm{Zu}$ nennen wären hier „Interkulturelle Vereine“ oder „Interkulturelle Begegnungsstätten“ oder auch prominente Beispiele wie die Türkische Gemeinde in Deutschland (TGD) oder der Verbund der sozial-kulturellen Migrantenvereine in Dortmund e. V. (VMDO). So ist das zentrale Anliegen des VMDO, ,eine lokale, partizipative, herkunftsunabhängige, kulturübergreifende und säkulare Orientierung " ${ }^{\text {57 }}$ aufzuweisen. Dies bedeutet

\footnotetext{
${ }^{57}$ https://www.vmdo.de/ueber-uns/ziele-und-selbstverstaendnis.html; vgl. für die Türkische Gemeinde in Deutschland (TGD): „Wir sind eine parteiübergreifende, säkulare und gemeinnützige Nichtregierungsorganisation und setzen uns ein für eine pluralistische und freiheitlich-demokratische Gesellschaft“", URL: https://www.tgd.de/wp-content/uploads/ 2019/06/Leitbild.pdf (letzte Abrufe am 03.04.2021).
} 
augenscheinlich jedoch nicht, dass Mitgliedervereine nicht auch religiöse (z. B. alevitische) Charakteristika aufweisen dürfen, sofern sie sich zu den verbandsintern propagierten Werten und Leitprinzipien bekennen. Die Betonung der Selbstverständnisse der Verbände als ,säkular“ ist dabei nicht zuletzt durchaus auch unter förderstrategischen Gesichtspunkten zu betrachten: Denn auf der Ebene des Bundes werden in Deutschland vorrangig säkulare MSO gefördert (Klie 2020) und der VMDO wie auch die TGD profitieren von projekt- sowie strukturbezogenen Förderungen (BT 2016b, 2018; vgl. auch Friedrichs 2020: 74).

\subsubsection{Zielgruppen der Aktivitäten, Aktivitätsradius der MSO}

\section{Zielgruppen der Aktivitäten}

83 Prozent der MSO für alle Zielgruppen offen

Mit einer deutlichen Überzahl gaben 83 Prozent der MSO unter der Bedingung nur einer zulässigen Antwort (Einfachnennung) an, dass ihre Aktivitäten und Angebote für alle offen sind. Dieses trifft auf säkulare und religiöse MSO in ungefähr gleichem Ausmaß zu (Differenz: 6 Prozentpunkte; Abb. 7.13). Bei 7 Prozent der MSO richten sich die Angebote und Dienstleistungen nur an Mitglieder, wobei sich auch hier keine wesentlichen Unterschiede zwischen den beiden Organisationstypen zeigen. Bei 4 Prozent - und ausschließlich bei religiösen MSO (14\%) - sind die Aktivitäten nur für Personen mit den gleichen religiösen Überzeugungen vorgesehen (Abb. 7.13). Unter ,Sonstiges“ verschriftlichten 5 Prozent der MSO, die mehrheitlich nicht-religiös sind, weitere Teilnahmekriterien, unter denen viermal die Anmerkung, dass ein (religiöser) Teil nur für Mitglieder (oder Gläubige) und ein anderer auch für Nicht-Mitglieder offen sei, zu finden war. Des Weiteren wurde in Einzelfällen beispielsweise festgehalten, dass sich die Aktivitäten ausschließlich an Mädchen und Frauen oder an Menschen der gleichen Muttersprache richten und ein Befragter schrieb auf Türkisch: „Wir kümmern uns um Straßentiere“.

Die Zielgruppenausrichtung der Organisationen ist ein wichtiger Anhaltspunkt für ihre Offenheit gegenüber der Umwelt und damit auch für den Öffentlichkeitsbezug der Aktivitäten. Sie ist zusammen mit Mitgliedschaftsvoraussetzungen und dem Kooperationsverhalten zu betrachten, um profunde Aussagen über Aufgeschlossenheit oder exklusive Tendenzen der MSO treffen zu können (s. hierzu 7.2.3). 


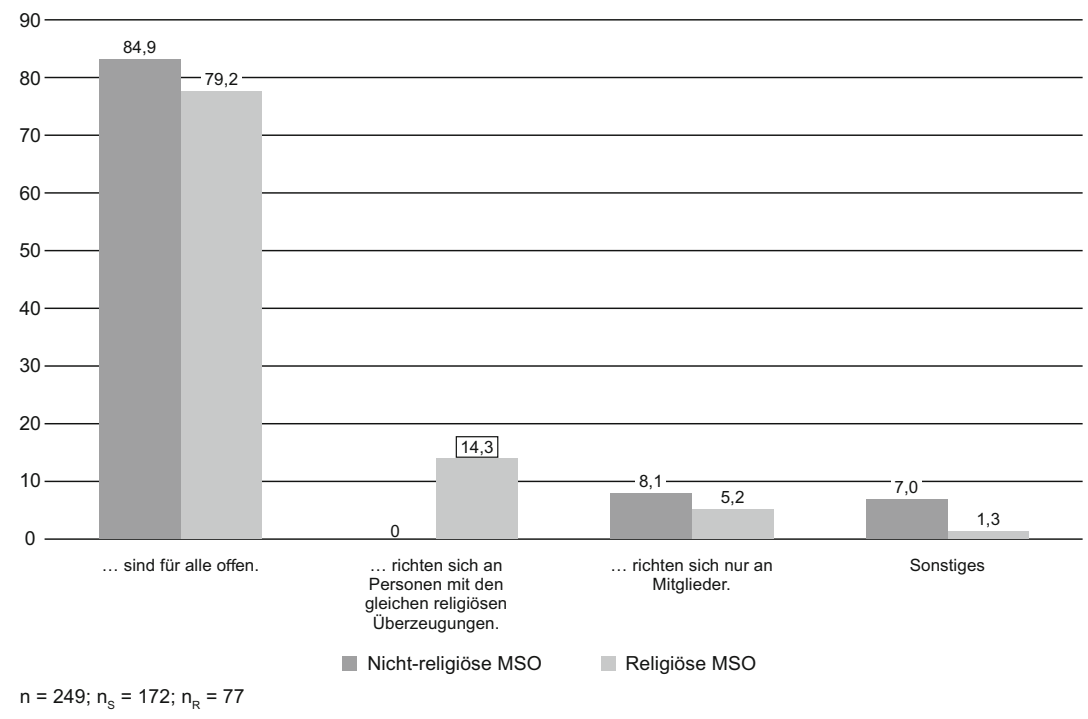

Abb. 7.13 Zielgruppen der Aktivitäten von (nicht-)religiösen MSO (in \%)

\section{Aktivitätsradien der MSO}

Bei der Auswertung der Beantwortung der geschlossenen Frage, in welchem Umfeld eine MSO aktiv ist, interessieren insbesondere der Radius bzw. die Kombinationen der insgesamt sieben vorgegebenen Wirkungsbereiche. Um einen Überblick zu erhalten und eine möglichst genaue Beschreibung vornehmen zu können, wurden die in diversen Mischungsverhältnissen genannten Bereiche abermals in eine nominalskalierte Variable überführt und Gruppen gebildet. Diese umfassen einzelne und in Kombination auftretende Aktivitätsradien. Insgesamt ist eine Vielfalt von Aktivitätsebenen-Verknüpfungen auszumachen (Tab. 7.24). So kann festgestellt werden, dass die MSO mit rund 17 Prozent in der Mehrzahl ausschließlich in der Stadt, lediglich 5 Prozent einzig im Stadtteil und 8 Prozent im Stadtteil und in der Stadt aktiv sind. 10 Prozent sind zugleich im Stadtteil, in der Stadt und Region (Kreis/Bezirk) und in dieser Gruppe mehrheitlich zudem auch in NRW aktiv. Über diese Konstellation, d. h. beim Stadtteil beginnend und über NRW hinausreichend, betätigen sich weitere 8 Prozent der MSO (Tab. 7.24). 
Fast 10 Prozent sind ausschließlich in den Konstellationen Stadt und Region oder Stadt und NRW (etwa hälftig) aktiv und weitere 10 Prozent entweder ausschließlich in der Region oder in Nordrhein-Westfalen (ebenfalls ungefähr hälftig). An einer dieser beiden Ebenen (Region oder NRW) ansetzend ist eine kleinere Gruppe über diese hinausgehend geschäftig (4\%). Gar nicht im Nahraum, sondern in Deutschland und/oder Europa und international unterwegs sind 8 Prozent der Vereinigungen, die Hälfte von diesen (4\%; in Tab. 7.24 nicht ausgewiesen) ist ausschließlich im internationalen Raum aktiv.

Weitere durch Gruppenbildungen abgebildete Aktivitätsradien sind insofern interessant, als sie Tätigkeiten im Nahraum, etwa im Stadtteil oder der Stadt umfassen, aber ggf. unter Auslassung der Ebenen ,Region“ oder ,NRW' in Deutschland, Europa und/oder international aktiv sind ( $7 \%$ und $7 \%$ ). In diesem Zusammenhang sind vereinzelt große „Ebenensprünge“ zu registrieren, die v. a. auch in die Gruppe „,sonstige Kombinationen“ (6\%) eingeordnet wurden (Tab. 7.24).

Tab. 7.24 Aktivitätsradien der MSO (gruppiert)

\begin{tabular}{lcc}
\hline & Häufigkeit & Prozent \\
\hline Nur Stadt & 43 & 16,9 \\
\hline Nur Stadtteil & 13 & 5,1 \\
\hline Stadtteil \& Stadt & 21 & 8,2 \\
\hline Stadtteil, Stadt, Region, (NRW) & 26 & 10,2 \\
\hline Stadtteil, Stadt, Region, NRW und darüber hinaus & 20 & 7,8 \\
\hline Nur Stadt \& Region oder nur Stadt \& NRW & 25 & 9,8 \\
\hline Nur Region oder nur NRW & 26 & 10,2 \\
\hline Region oder NRW und darüber hinaus & 10 & 3,9 \\
\hline Stadtteil, Stadt, (NRW) und darüber hinaus & 17 & 6,7 \\
\hline Stadt, (Region), NRW und darüber hinaus & 18 & 7,1 \\
\hline D (und/oder) EU, International & 21 & 8,2 \\
\hline Sonstige Kombinationen & 15 & 5,9 \\
\hline Gesamt & 255 & 100,0
\end{tabular}

In Bezug auf die Unterteilung der MSO in (nicht-)religiöse zeigt ein Prozentwertvergleich in einer Kreuztabelle, dass beide Organisationskategorien sehr ähnliche Aktivitätsradien aufweisen. Religiöse MSO sind etwas häufiger im Stadtteil und in der Stadt aktiv (RMO: $14 \%$ vs. MO: $6 \%$ ).

Die Studie des SVR-Forschungsbereichs (Friedrichs et al. 2020: $17 \mathrm{f}$.) hat für die MSO in den vier Bundesländern Nordrhein-Westfalen, Bayern, Sachsen und Berlin festgestellt, dass die MSO mit 61 Prozent hauptsächlich in der eigenen Stadt 
bzw. im eigenen Stadtbezirk und damit doppelt so häufig in diesen Bereichen aktiv sind als die im Rahmen dieser Arbeit befragten MSO (ca. 30 \%; Tab. 7.24). ${ }^{58}$ Bei allen Einschränkungen bezüglich der Vergleichbarkeit der Studienergebnisse kann mit Blick auf die Ergebnisse für NRW vorsichtig geschlussfolgert werden, dass die MSO in diesem Bundesland auch auf anderen gesellschaftlichen Ebenen besonders aktiv sind.

\section{- Interpretation der Ergebnisse}

Große Bedeutung von Beziehungen im nahen, fernen und ,intermediären` Raum

Die diversen Aktivitätsradien implizieren das Vorhandensein unterschiedlicher Reichweiten und Formen von Beziehungen, Verbundenheit und (ggf. als verpflichtend betrachteter) Unterstützung von und zwischen Individuen und Gruppen. Weiter gedacht verweist die Manifestation von unterschiedlich weitreichenden Beziehungen auf verschiedene Formen von Sozialkapital, die auf divergierenden Niveaus als wechselseitige Hilfeleistungen, Kooperationen und soziales Vertrauen zwischen den Bezugsgruppen in Erscheinung treten können. Diese sind in Bezug auf die jeweiligen (Ein-)Wirkungsbereiche nicht als trennscharfe Größen, sondern auf einem Kontinuum zwischen den extremen Endpolen des sozialen nachbarschaftlichen Nahraums und des transnationalen Fernraums zu denken. Zu bedenken ist, dass das gleichzeitige Aktivsein sowohl vor Ort als auch in der Ferne im Ausland als eine beachtliche Leistung einzustufen ist, die bestimmte gesellschaftspolitische Einstellungen der in den Selbstorganisationen involvierten Personen und das Vorhandensein von sozialen und ökonomischen Ressourcen voraussetzt; neben Kapitalausstattungen erfordert es darüber hinaus aber auch spezifische Dispositionen wie etwa ein Verlangen nach Mitgestaltung oder den Willen, als notwendig betrachtete Veränderungen herbeizuführen und auch öffentliche Rollen wahrzunehmen.

In diesem Zusammenhang kann angeknüpft werden an den - in modifizierter Form auch für den Forschungsgegenstand der MSO fruchtbar zu machenden - Vorschlag einer konzeptuellen Differenzierung von drei Typen von Solidarität, die in verschiedenen Formen und auf unterschiedlichen Ebenen erbracht werden (Blinkert/Klie 2004; 2018: 410 ff.). Der vorgeschlagenen Dreiteilung zufolge bezieht sich 1.) Nahraumsolidarität auf den sozialen Nahraum und auf Mitglieder primärer, gut gekannter sozialer Gruppen, zu denen enge Beziehungen bestehen; 2.) Fernraumsolidarität verweist auf den sozialen Fernraum und erreicht lediglich partiell oder nicht

\footnotetext{
${ }^{58}$ Frage in der SVR-Studie: „Wo ist Ihre Organisation hauptsächlich aktiv?“; Antworten: in der Stadt/im Stadtbezirk: $61 \%$; in mehreren Städten/Gemeinden: $14 \%$; im gesamten Bundesland: $8 \%$; in mehreren Bundesländern: $4 \%$; in ganz Deutschland: $5 \%$; international: $7 \%$ (Friedrichs et al. 2020: 18).
} 
bekannte Menschen, über deren spezifische Lebenssituationen nur wenig gewusst wird; 3.) in einem intermediären Bereich erbrachte solidarische Leistungen adressieren in einem relativ nahen sozialen Umfeld lebende, nur wenig oder gar nicht bekannte Menschen.

In Hinblick auf die innerhalb der Konzeptualisierung zum Teil angenommene Unbekanntheit der adressierten Personen könnte im Falle der MSO allerdings im Rahmen der im Fernraum oder im intermediären Bereich vollzogenen Unterstützung durchaus auch von Bekannten, Freunden und Verwandten ausgegangen werden.

Obgleich eine trennscharfe Abgrenzung der drei Solidaritätsformen schwierig sein sollte (wann endet die eine Form, wann fängt die andere an?) und zwischen ihnen bestehende Zusammenhänge noch unerforscht sind, scheint es für die Einordnung der vielfältigen Aktivitätsradien der MSO fruchtbar gemacht werden zu können, indem die auf unterschiedlichen Ebenen zu verortenden regelmäßigen Interaktionen und Kommunikationen der MSO in die Dreier-Konzeptualisierung eingeordnet werden. Der Begriff der Solidarität, der ein starkes Zusammengehörigkeitsgefühl suggeriert, das nicht notwendigerweise vorhanden sein muss, könnte dabei durch den neutraleren Terminus „Beziehung(en)“ ausgetauscht werden.

So kann der Begriff der „Nahraumbeziehungen“ - im Rahmen dieser Studie - für die im Stadtteil und in der Stadt gepflegten Verhältnisse geltend gemacht werden. Die Region (Kreis/Bezirk) sowie das Bundesland Nordrhein-Westfalen stellen den „,intermediären Bereich“ dar, innerhalb dessen soziale Verbindungen bestehen. Der Terminus der „Fernraumbeziehungen“ kann auf die innerhalb von Deutschland, in Europa oder international bestehenden Kontakte der MSO angewandt werden.

Die einzelnen und unterschiedlichen (Misch-)Formen von Beziehungen - oder zugespitzt: von Sozialkapital - sollten insbesondere für migrantische Selbstorganisationen bedeutsam sein. Daher sollte ihnen zukünftig im Kontext der vielfach thematisierten transnationalen Ausrichtungen von MSO (Pries/Sezgin 2010a; Pries 2001) und deren Verbindungen zu Diaspora-Standorten (Nagel 2013a; Lauser/Weißköppel 2008) mehr Aufmerksamkeit geschenkt und Konturen gegeben werden.

\subsubsection{Kooperationen: Partner, Ziele, wahrgenommene Schwierigkeiten}

\section{Kooperationspartnertypen}

Den Angaben der Funktionsträger zufolge arbeiten mehr als drei Viertel der MSO (76\%) gegenwärtig mit anderen Organisationen zusammen oder haben dieses in 
den letzten fünf Jahren getan. Unter den kooperierenden MSO sind religiöse (78 \%) und nicht-religiöse ( $76 \%$ ) zu fast gleichen Anteilen vertreten.

In möglichen Mehrfachantworten markiert werden sollten bestimmte Typen von Organisationen, mit denen zusammengearbeitet wird oder wurde. Vorgegeben waren 14 Items (inklusive „Sonstige“). Festgestellt wird dadurch also nicht die genaue Anzahl der Kooperationspartner insgesamt, sondern die Anzahl der Organisationsarten, mit denen zusammengearbeitet wird.

Städtische Einrichtungen, MSO und ortsansässige Vereine als häufigste Kooperationspartner

Die Abb. 7.14 verdeutlicht die Prävalenz von drei Organisationen: Organisationen/Ämter in der eigenen Stadt (68\%), wie beispielsweise das Sozialoder Jugendamt, Schulen, der Integrationsrat oder das Kommunale Integrationszentrum, andere Migrantenselbstorganisationen (67\%) sowie ortsansässige Vereine $(61 \%)$. Von rund 39 bis 35 Prozent werden Wohlfahrtsverbände (39\%), Integrationsagenturen (35\%) und Kirchen (35\%) als Partner im Rahmen bestimmter gemeinsamer Unternehmungen ausgewiesen. Jeweils rund ein Viertel arbeitet mit Universitäten (26\%) oder staatlichen Organisationen (26\%), wie z. B. einem Bundes- oder Landesministerium zusammen, etwas weniger mit Stiftungen (22\%), Ämtern oder Einrichtungen in einer anderen Stadt

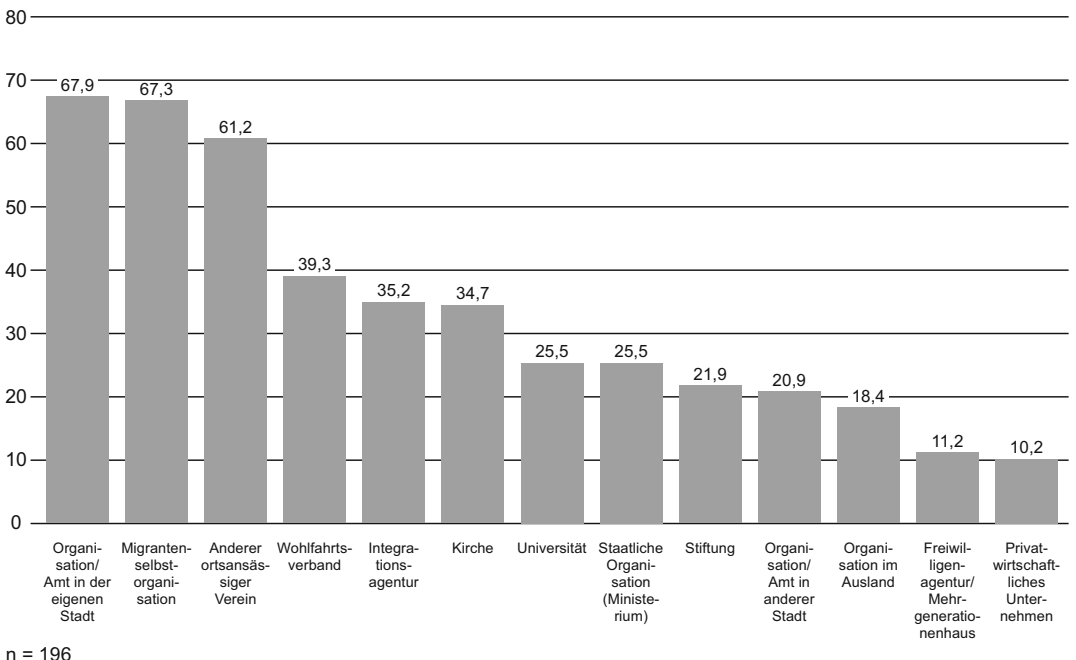

Abb. 7.14 Kooperationspartner der MSO (in \%) 
(21\%) oder im Ausland ansässigen Organisationen (18\%). Freiwilligenagenturen oder Mehrgenerationenhäuser $(11 \%)$ wie auch privatwirtschaftliche Unternehmen (10\%) stellen seltenere Kooperationspartner dar.

Interessanterweise zeigen die Befunde zum Kooperationsverhalten der MSO insgesamt tendenziell viele Ähnlichkeiten mit den Ergebnissen der Studie des SVR-Forschungsbereiches, der zufolge 65 Prozent der in den vier Bundesländern Nordrhein-Westfalen, Bayern, Sachsen und Berlin befragten MSO mit Stadtverwaltungen, 64 Prozent mit anderen gemeinnützigen Vereinen und 61 Prozent mit anderen MSO ,partnerschaftlich“ zusammenarbeiten. 30 Prozent kooperieren mit Wohlfahrtsverbänden ${ }^{59}$ (Friedrichs et al. 2020: 59; I. 2.1).

Ein Blick auf die jeweils für religiöse und säkulare MSO relevanten Kooperationspartner zeigt deutliche Unterschiede. So zeigt sich, dass nicht-religiöse MSO wesentlich häufiger mit Organisationen oder Ämtern in der eigenen Stadt (MO: 78 \% vs. RMO: $47 \%$ ), aber auch mit staatlichen Organisationen (z. B. Bundes- oder Landesministerien), mit Universitäten und Wohlfahrtsverbänden wie auch Stiftungen, Integrationsagenturen (Differenzen: 18 bis 16 Prozentpunkte), Freiwilligenagenturen bzw. Mehrgenerationenhäusern, Migrantenselbstorganisationen und privatwirtschaftlichen Unternehmen zusammenarbeiten (Differenzen: 14 bis 13 Prozentpunkte). Für religiöse MSO bedeutsame Kooperationspartner sind Kirchen (RMO: 50 \% vs. MO: $28 \%$ ).

\section{- Statistische Tests und Ergebnisinterpretation}

Säkulare MSO haben deutlich vielfältigere Kooperationspartner

Anhand der Berechnung des Koeffizienten Cramers $V$ zeigen sich für den Kooperationstypus „Organisation oder Amt in der eigenen Stadt“ ein signifikanter mittelstarker Zusammenhang und für acht der oben genannten Kooperationstypen signifikante schwache Zusammenhänge ${ }^{60}$. Allein für die Kooperationspartner ,Migrantenselbstorganisation“, „Organisation/Amt in einer anderen Stadt“, „Anderer ortsansässiger Verein“" und „,Organisation im Ausland“ zeigen sich keine Signifikanzen. Für die beiden MSO-Typen ist somit von einem signifikant unterschiedlichen

\footnotetext{
${ }^{59}$ Laut der SVR-Studie sind weitere relevante Kooperationspartner Bezirksverwaltungen und die Landespolitik (jeweils $27 \%$ ), Stiftungen, Hochschulen und informelle Initiativen (jeweils $24 \%$ ), die Bundespolitik (17\%) sowie Behörden und Organisationen (15\%) (Friedrichs et al. 2020: 59).

${ }^{60}$ Organisation/Amt in der eigenen Stadt (Cramers $\left.V=0,307 * * *\right)$; Staatliche Organisation und Universität (Cramers $\left.V=0,197^{* *}\right)$; Wohlfahrtsverband $\left(V=0,165^{*}\right)$; Stiftung $(V=$ $0,202 * *)$; Integrationsagentur $(V=0,157 *)$; Freiwilligenagentur bzw. Mehrgenerationenhaus $\left(V=0,207^{* *}\right)$; privatwirtschaftliches Unternehmen $\left(V=0,193^{* *}\right)$; Kirche $\left(V=0,219^{* *}\right)$.
} 
Kooperationsverhalten auszugehen, das darauf hindeutet, dass unter den beteiligten Akteuren jeweils spezifische Partner präferiert oder begünstigt werden.

Eine Aufsummierung von gekennzeichneten Organisationstypen zeigt, dass in einer Marge von 14 bis 19 Prozent unter den MSO am häufigsten mit 2 bis 5 Organisationsarten zusammengearbeitet wird (Tab. 7.25). Über die Angaben der 196 kooperierenden MSO hinweg zeigt sich vermittels einer Überführung der Informationen in eine nominalskalierte Variable eine Variationsbreite von Kooperationspartner-Kombinationen, die keine dominanten Häufigkeiten erkennen lassen und folglich keine Gruppierungen ermöglichen.

Tab.7.25 Anzahl von Kooperationstypen

\begin{tabular}{ccc}
\hline Anzahl & Häufigkeit & Prozent \\
\hline 1 & 15 & 7,7 \\
\hline 2 & 39 & 19,9 \\
\hline 3 & 30 & 15,3 \\
\hline 4 & 26 & 13,3 \\
\hline 5 & 32 & 16,3 \\
\hline 6 & 15 & 7,7 \\
\hline 7 & 13 & 6,6 \\
\hline 8 & 9 & 4,6 \\
\hline 9 & 6 & 3,1 \\
\hline 10 & 7 & 3,6 \\
\hline 11 & 1 & 0,5 \\
\hline 12 & 2 & 1,0 \\
\hline 13 & 1 & 0,5 \\
\hline Gesamt & 196 & 100,0 \\
\hline
\end{tabular}

Anhand einer bivariaten Tabellierung (hier nicht ausgewiesen) wird deutlich, dass die religiösen mit 26 und 19 Prozent am häufigsten mit zwei bzw. drei Organisationstypen zusammenarbeiten sowie zu jeweils 13 Prozent mit vier oder - und damit unter allen MSO mehrheitlich - mit nur einem Kooperationstypus ${ }^{61}$. Hingegen kooperieren die säkularen MSO am häufigsten mit zwei (17 \%) oder fünf

\footnotetext{
${ }^{61}$ Als einzelne, ausschließliche Kooperationspartnertypen wurden folgende genannt: 8-mal ortsansässiger Verein; 2-mal Organisation/Amt in der eigenen Stadt; einmal Kirche; einmal christliches Hilfswerk; einmal „Sonstiges“ (Verein in Nachbarstadt).
} 
(16\%) unterschiedlichen Institutionen, gefolgt von der Zusammenarbeit mit vier und drei Einrichtungsarten (jeweils $13 \%$ ). Des Weiteren ist erkennbar, dass säkulare Vereinigungen öfters mit sechs und mehr Organisationstypen zusammenarbeiten; die Anzahl von zehn bis 13 wird ausschließlich von nicht-religiösen Vereinigungen angegeben.

Der $t$-Test zeigt, dass säkulare MSO mit einer signifikant größeren Anzahl von Organisationstypen kooperieren. Die Effektstärke ist als mittel einzuschätzen. ${ }^{62}$ Das für nicht-religiöse MSO auszumachende breitere Spektrum an Kooperationspartnern verweist auf deren aktiveres Kooperationsverhalten. Dieses kann auf die Verfügbarkeit größerer Netzwerke und die bereits erwähnte Präferenz oder Bevorteilung bestimmter Partnerorganisationen zurückzuführen sein. Auch kann die strategische Verfolgung spezifischer Anliegen eine Rolle spielen, die zum Aufbau des Netzwerks führt, aber ggf. auch erst aus ihm resultiert. Die kleineren Netzwerke der religiösen Vereinigungen könnten potenziell auf die Existenz einzelner starker Kollaborationen zurückzuführen sein, die weitere überflüssig erscheinen lassen, da Bedarfe abgedeckt sind. Damit einhergehend könnte eine größere Selbstgenügsamkeit oder ,habituelle Zurückgenommenheit“ (Nagel 2015b: 29) in den religiösen MSO von Bedeutung sein, die in einem geringer ausgeprägten Bestreben nach Zusammenarbeit zwecks spezifischer Vorteile oder Mehrwerte resultiert. Diese Annahme lässt sich gut vereinbaren mit dem primären Selbstverständnis der religiösen MSO als Religionsgemeinschaft und Gemeinschaft Gleichgesinnter gegenüber seitens der säkularen Vereinigungen signifikant stärker befürworteten Selbstdefinitionen, die proaktive, die Entwicklung eines Zustands mitbestimmende und herbeiführende Verhaltensweisen implizieren (Förderorganisation, Netzwerk; 7.1.3). Andererseits könnte es auch sein, dass keine passenden Kooperationspartner gefunden werden.

Ganz grundsätzlich ist an dieser Stelle anzumerken, dass eine Zusammenarbeit von MSO mit unterschiedlichen Organisationen die Bereitschaft und Offenheit aller beteiligten Akteure voraussetzt und es sich nicht um eine seitens der MSO zu erfüllende Bringschuld oder Anforderung handelt. Ein Nicht- oder Wenig-Kooperieren kann mehrere Ursachen haben (II. 3.2; II. 4.3.5; II. 4.5).

\footnotetext{
${ }^{62}$ Varianzhomogenität lag mit $F(6,506) ; p=0,012$ nicht vor. Im durchgeführten WelchTest wurde der Gruppenunterschied mit $t(161,574)=4,23 ; p=0,000$ signifikant; Cohen's $d=0,57$.
} 


\section{- Hypothesenprüfung und Ergebnisdiskussion}

Mit Blick auf das Kooperationsverhalten, das Aktivitätsspektrum sowie die personellen und finanziellen Ausstattungen der MSO ist schließlich ebenfalls von Interesse und zu prüfen, inwieweit zwischen der Verfügbarkeit dieser Ressourcen und der Leistungsfähigkeit der Vereinigungen proportionale Zusammenhänge vorzufinden sind. Angenommen wird, dass eine Organisation umso mehr Leistungen erbringen kann, je ressourcenstärker sie ist (II. 4.3.2). Daher wurde folgende Hypothese formuliert:

H2: Insbesondere die mit genügend Geld und personellen Ressourcen (Mitglieder, Hauptamtliche und Engagierte) ausgestatteten MSO sind imstande, ein breites Spektrum an Aktivitäten und Dienstleistungen anzubieten und Kooperationen einzugehen.

Die Prüfung der Hypothese erfolgte mit multiplen linearen Regressionen, wobei die Gesamtzahl der Aktivitäten und die Gesamtzahl der Kooperationspartner in zwei separaten Modellen jeweils als abhängige Variablen (kurz: AV) fungierten. ${ }^{63}$ Hinsichtlich der Aktivitäten zeigt sich überraschender Weise kein einziger signifikanter Effekt. Bei dem finalen Modell ergab sich ein korrigiertes Bestimmtheitsmaß (korrigiertes $\mathrm{R}^{2}$ ) von 0,031 . Somit werden lediglich 3 Prozent der Varianz der Aktivitäten durch das aufgestellte Modell erklärt.

Im Modell mit der Anzahl der Kooperationspartner als AV zeigten sich zwei signifikante Effekte. Zum einen ist der Einfluss von Einrichtungen mit 11 bis 50 Mitgliedern im Vergleich zu jenen mit höchstens 10 Mitgliedern signifikant größer $(\beta=1,7 ; p=0,012)$; zum anderen hat die Quantität der Hauptamtlichen einen signifikant positiven Einfluss auf die Quantität der Kooperationspartner $(\beta=0,15 ; p=$ 0,$000 ; \mathrm{n}=161$ ). Somit haben MSO mit 11 bis 50 Mitgliedern sowie MSO mit einer höheren Hauptamtlichenanzahl ein signifikant breiteres Spektrum an Kooperationspartnern ${ }^{64}$. Dabei ergab sich ein korrigiertes Bestimmtheitsmaß (korrigiertes $\mathrm{R}^{2}$ )

${ }^{63}$ Anmerkung zu den Berechnungen der Regressionen: Bei den Regressionen zeigten sich Cook-Distanzen deutlich größer 1. Diese wurden entfernt und die Regressionen erneut durchgeführt bis keine weiteren einflussreichen Fälle vorlagen (6.2).

${ }^{64}$ In beide finale Regressionsmodelle wurden als unabhängige Variablen (quasi-)metrische Variablen aufgenommen (Einschluss-Methode). Diese betreffen die Anzahl von Hauptamtlichen und Engagierten sowie die (Nicht-)Zustimmung zu den beiden Aussagen, dass im Allgemeinen genügend Freiwillige (Engagierte) gewonnen sowie ausreichend Freiwillige für unbezahlt ausgeführte Leitungspositionen und Ämter (,Ehrenämter“) gefunden werden können. Die Existenz finanzieller Engpässe wurde mit einer dichotomen Variablen berücksichtigt (Codierung $0 / 1=$ nicht vorhanden/vorhanden). 
von 0,21. Somit werden 21 Prozent der Varianz der Anzahl der Kooperationspartner durch das aufgestellte Modell erklärt.

Anhand der multiplen Regressionen zeigt sich also, dass die personelle Ressourcenausstattung in Form der Existenz von Hauptamtlichen ein relevanter Einflussfaktor für das Kooperationsverhalten, nicht aber für die Summe der (nicht-)religiösen Aktivitäten der MSO ist. Die Verfügbarkeit von personellen Ressourcen, konkret Hauptamtlichen, scheint somit keine signifikante Einwirkung auf den Tätigkeitsumfang der MSO zu haben. In Hinblick auf das Kooperationsverhalten sowie das Aktivitätsspektrum der MSO stellen sowohl eine als (nicht) ausreichend empfundene finanzielle Ressourcenausstattung als auch die Anzahl der Engagierten keine signifikanten Einflussgrößen dar. Im Gegensatz zu diesen Befunden konnten die Autoren der Studie Islamisches Gemeindeleben in Deutschland (Halm et al. 2012a: 7, 117) die Ausstattung der Organisationen mit personellen, infrastrukturellen und finanziellen Ressourcen, im Sinne auch des Vorhandenseins von Räumlichkeiten, einzelnen Abteilungen und Immobilienbesitz, als zentrale Einflussfaktoren auf die Angebotsvielfalt identifizieren. Allerdings konstatieren auch Halm et al., dass die Ressourcen zwar eine notwendige, allerdings nicht hinreichende Bedingung für die Erfüllung von Aufgaben sind (ebd.; II. 4.3.2). An dieser Stelle ist - wie bereits im Rahmen der Auswertung der Tätigkeitsbereiche geschehen (7.1.4) - jedoch nochmals kritisch anzumerken, dass bei der Angabe von Aktivitäten durch die Funktionsträger sozial erwünschtes und ein die Aktivitäten der MSO überschätzendes Antwortverhalten nicht auszuschließen ist. Gemäß dem Motto „Das haben wir auch schon mal gemacht" könnte die konkrete Frage nach den Schwerpunkten der Aktivitäten und Dienstleistungen aus dem Blick geraten und zu großzügig beantwortet worden sein. Insofern könnte eine den tatsächlichen Gegebenheiten entsprechende und damit valide Verhältnismäßigkeit zwischen der Anzahl der Aktivitäten und der Anzahl der Hauptamtlichen für die Berechnung der Regressionen unter den genannten Umständen nicht gegeben sein.

Für die beiden Variablen „Gewinnung genügend Freiwilliger“ und „Leitungspositionen“ erfolgte eine Recodierung $(4=1 ; 3=2 ; 2=3 ; 1=4)$. Die fehlenden Werte der Variablen zur Anzahl von Engagierten und Ehrenamtlichen wurden dann „0“ gesetzt, wenn die Existenz von Hauptamtlichen verneint wurde.

Die Kategorien der ordinalskalierten Variable zur Mitgliederzahl (,unter 10‘; ,11 bis 50‘; ,51 bis 100‘; , mehr als 100‘) wurden als Dummy-Variablen ins Modell integriert. Für die Modellierung des Einflusses einer kategorialen Variablen mit c Kategorien werden c minus 1 Dummy-Variablen in das Modell aufgenommen (Farhmeir et al. 2009: 80-83). Dabei fungiert die ausgelassene Kategorie als Referenzkategorie (hier: ,unter 10 Mitglieder"). Das bedeutet, dass der Effekt der nicht ausgelassenen Kategorien immer mit der Referenzkategorie verglichen wird. 


\section{Ziele der Kooperationen mit anderen Organisationen}

Bei der Frage nach mit den Kooperationen verbundenen wichtigen Zielen waren neun Items hinsichtlich ihrer Relevanz für die kooperierenden MSO einzustufen. Es zeigt sich, dass dem Informationsgewinn und -austausch (96\%), dem Aufbau einer langfristigen Zusammenarbeit $(94 \%)$, der Verbesserung des Kontaktes zu Zielgruppen $(93 \%)$ wie auch der Steigerung der Bekanntheit und Vernetzung der Organisation (88 \%) die wichtigste Bedeutung zukommen (Abb. 7.15). Der letztgenannte Aspekt (Bekanntheit/Vernetzung) trifft um rund 15 Prozentpunkte für die nicht-religiösen Organisationen häufiger zu (MO: $93 \%$ vs. RMO: $78 \%$ ).

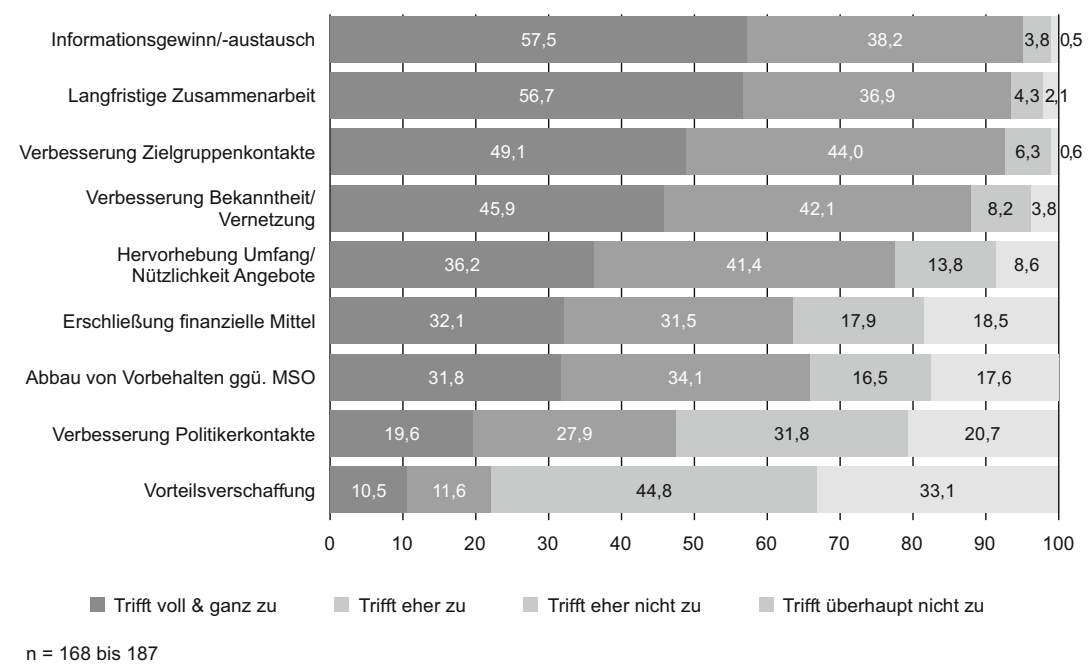

Abb.7.15 Kooperationsziele der MSO (in \%)

Der Hervorhebung des Umfangs und der Nützlichkeit der Angebote (78 \%), dem Abbau von wahrgenommenen Vorbehalten gegenüber der Organisation (66\%) und der Erschließung von zusätzlichen finanziellen Mitteln (64 \%) wurden mit jeweils über 60 Prozent zugestimmt und damit ebenfalls als wichtig erachtet. Der Zufluss von finanziellen Ressourcen sowie die Betonung von Umfang und Wert der Aktivitäten besitzen für die religiösen Vereinigungen jedoch eine um 24 bzw. 12 Prozentpunkte geringere Bedeutung als für die säkularen Organisationen.

Die Verbesserung von Kontakten zu Politikern ist für fast die Hälfte der MSO (48\%) bedeutsam, für die religiösen MSO (38\%) allerdings in einem geringeren 
Ausmaß als für die nicht-religiösen (52\%); circa ein Fünftel der kooperierenden MSO (22\%) strebt an, sich durch Zusammenarbeit einen Vorteil gegenüber anderen Organisationen zu verschaffen.

\section{- Statistische Tests}

Breitere und taktischere Ausrichtung der Kooperationsarbeit unter den säkularen MSO

Ergebnisse des $U$-Tests zeigen für 6 Kooperationsziele eine jeweils statistisch signifikante größere Bedeutung für säkulare Vereinigungen. Die 6 Zwecke betreffen die Verbesserung der Bekanntheit und Vernetzung der Organisation, den Erhalt von Geldern, den Informationsgewinn, die Etablierung einer langfristigen Zusammenarbeit, die Verbesserung von Kontakten zu Zielgruppen sowie die Hervorhebung der Nützlichkeit der Angebote der MSO (s. Tab. A 4 im elektronischen Zusatzmaterial). Dies verweist auf eine im Vergleich zu den religiösen MSO grundsätzlich breitere und taktischere Ausrichtung der Kooperationsarbeit der säkularen Vereinigungen. Dafür spricht auch die größere Vielfalt ihrer Kooperationspartner. Die religiösen Vereinigungen scheinen im Rahmen ihrer Kooperationsanliegen hingegen weniger ambitioniert und zurückhaltender zu sein. Potenziell zugrundeliegende Ursachen wurden oben erörtert (kleine, aber starke Netzwerke; weniger Bedarfe oder ein Mangel an adäquaten Kooperationspartnern).

\section{- Hypothesenprüfung und Ergebnisdiskussion}

Die zentrale Bedeutung öffentlicher Bekanntheit und Vernetzung für MSO

Im Rahmen der Darlegung der theoretischen Annahmen des soziologischen Neoinstitutionalismus (NI) (II. 4.5) wurde mit Blick auf zentrale Kooperationsziele eine im Folgenden zu überprüfende Hypothese formuliert.

Der NI stellt den ökonomische Effizienzperspektiven dominierenden Leitgedanken von Legitimationserfordernissen heraus: Nur wenn eine Organisation Legitimität gewonnen hat und bewahrt, können ihr Anerkennung, Wertschätzung und Unterstützung zuteilwerden, beispielsweise in Form von personeller oder finanzieller Ressourcenzufuhr, die letztlich ihre Überlebensfähigkeit, Stabilität und ihren Erfolg sichern (Hellmann 2006: 75; Meyer/Rowan 1991: 53). Ein zentrales Anliegen von Organisationen ist es daher, diesen Legitimitätsstatus - im Sinne von Integrität, Vertrauenswürdigkeit und Anerkennung - zu erlangen. Sie operieren und präsentieren sich daher entsprechend, um seitens für sie zentraler Akteure und Anspruchsgruppen Wertschätzung, Zustimmung und Unterstützung zu erhalten. An dieser theoretischen Prämisse aufsetzend, wurde folgende Hypothese formuliert: 
H6: Verglichen mit anderen Anliegen stellen v. a. die Hervorhebung des Umfangs und der Nützlichkeit der Aktivitäten, die Steigerung der Bekanntheit und Vernetzung der MSO, der Abbau von wahrgenommenen Vorbehalten sowie die Verbesserung der Kontakte zu Politikern zentrale Kooperationsziele dar.

Auf den ersten Blick scheinen die Untersuchungsergebnisse die Hypothese zunächst zu falsifizieren. Denn die den Legitimitätsstatus und die Anerkennung der MSO betreffenden und daher im Rahmen von $\mathbf{H 6}$ als besonders bedeutsam eingestuften Zwecke nehmen unter allen Kooperationszielen die mittleren bzw. unteren Ränge ein (s. auch Abb. 7.15). Vielmehr scheint ein durch die Zusammenarbeit mit anderen Organisationen angestrebtes spezifisches Qualitätsniveau bzw. eine Qualifizierung der eigenen Arbeit im Vordergrund zu stehen. Diese umfasst die Aspekte von Wissensaneignung, Zielgruppenzugängen und Nachhaltigkeit der Kooperationen.

Die Durchführung einer EFA zeigt eine Zwei-Faktoren-Lösung an (Tab. 7.26), die die soeben explizierten Kooperationszwecke spiegelt (Faktor 1: „Wettbewerbsorientierte Besserstellung und Legitimitätsgewinn der MSO“; Faktor 2: „Qualifizierung und Ausweitung der Arbeitsbereiche“).

Tab.7.26 Rotierte Faktorladungsmatrix zu Kooperationszielen

\begin{tabular}{lrr}
\hline & \multicolumn{2}{c}{ Faktoren } \\
\hline Erschließung von zusätzlichen finanziellen Mitteln & $\mathbf{1}$ \\
\hline Verschaffung eines Vorteils gegenüber anderen Organisationen &, 767 &, 179 \\
\hline Verbesserung der Kontakte zu Politikern &, 686 &, 020 \\
\hline Hervorhebung von Umfang und Nützlichkeit unserer Angebote &, 676 &, 224 \\
\hline Verbesserung der Bekanntheit und Vernetzung unserer Organisation &, 640 &, 331 \\
\hline Abbau von Vorbehalten gegenüber unserer Organisation &, 615 &, 505 \\
\hline Verbesserung des Kontaktes zu Zielgruppen unserer Arbeit &, 608 &, 106 \\
\hline Informationsgewinn und Informationsaustausch &, 108 &, 832 \\
\hline Aufbau von langfristiger Zusammenarbeit &, 121 &, $\mathbf{8 1 4}$ \\
\hline
\end{tabular}

Hauptkomponentenanalyse. Fehlende Werte: paarweiser Fallausschluss. Rotationsmethode: Varimax. KMO-Test: 0,$830 ; \mathrm{n}=219-241$.

Die Faktorladungsmatrix (Tab. 7.26) zeigt, dass die theoretisch verankerte Bündelung und in der Hypothese vorgenommene Berücksichtigung von vier Kooperationszielen (Tab. 7.26, Kursivsetzungen) durch die ebenfalls auf den Faktor 1 ladenden zwei Items „Erschließung von zusätzlichen finanziellen Mitteln“ sowie „Verschaffung eines Vorteils gegenüber anderen Organisationen“ flankiert werden. Dies ist als ein Indiz für zu eng gefasste theoretische Annahmen zu deuten. Denn 
das Konkurrenzmotiv wie auch ökonomische Zwecke wurden im Kontext des im NI betonten primären Ziels der Statuserhöhung als nachrangig eingestuft.

Eine gesonderte Beachtung verdient in diesem Zusammenhang jedoch die Querladung des Items „Verbesserung von Bekanntheit und Vernetzung“. Sie ist ein Anhaltspunkt für seine Zwitterstellung und kann als eine wesentliche Voraussetzung für die praktische Verwirklichung der auf die Faktoren 1 und 2 ladenden Ziele betrachtet werden. Damit stellt die Querladung ein Indiz - ein Einfallstor - für die Bestätigung der Annahmen des NI dar: Die Erlangung von öffentlicher Bekanntheit (bzw. Aufmerksamkeit) wie auch Vernetzungen mit anderen Organisationen können als eine zentrale Bedingung für die Realisierung der anderen Kooperationsziele eingestuft werden. Es kann angenommen werden, dass öffentliches Gekanntwerden (auch im weiteren Sinne von Popularität) und soziale Kontakte für kooperationswillige MSO grundsätzlich von Bedeutung sind. Insofern sind Kooperationsziele hinsichtlich des Gewinns von Anerkennung und Legitimität nicht trennscharf zu fassen, sondern in fließenden Übergängen miteinander verquickt. Angesichts der Verknüpfung von Kooperationszwecken legt diese Interpretation nahe, dass die im NI veranschlagte Statuserhöhung im Rahmen von mit Kooperationen verfolgten Zielen immer in irgendeiner Weise relevant ist, sich allerdings in ungleichen Intensitätsgraden und Handlungsorientierungen niederschlägt. Auf die vielfältigen und heterogenen Ausrichtungen bzw. Anliegen der MSO wurde im Rahmen dieser Arbeit bereits mehrfach verwiesen. Der Bedarf, Beachtung und ein positives Image zu erhalten, wird dabei für die migrantischen Vereinigungen disparat ausfallen und entsprechend mit unterschiedlichen Vorgehensweisen und Kontakt(aufnahme)strategien einhergehen. ${ }^{65}$

\footnotetext{
65 Anzunehmen wäre, dass die Thesen des NI insbesondere für die im öffentlichen und politischen Interesse stehenden islamischen Verbände von Bedeutung sein sollten, da sie als Ansprechpartner und Interessenvertreter der organisierten Muslime in Deutschland wahrgenommen werden und eine Gleichstellung mit den christlichen Religionsgemeinschaften im deutschen Religionsverfassungsrecht einfordern (Rosenow-Williams 2012; II. 4.5). Aufgrund spezifischer politischer Entwicklungen und brisanter Ereignisse sind sie in den letzten Jahren jedoch zum Teil in die Defensive geraten und haben daher ihr angeschlagenes Image aufzupolieren.

Ergebnisse des $U$-Tests (s. Tab. A 4 im elektronischen Zusatzmaterial) haben hinsichtlich der in der Hypothese berücksichtigten Ziele keine höhere Bedeutung für religiöse MSO ermittelt. Ein genauerer Blick auf das Antwortverhalten der islamischen Verbände zeigt für DİTİB, IGMG, IGBD und BDAJ in Bezug auf die drei Ziele ,Verbesserung Bekanntheit/Vernetzung“, „Hervorhebung Umfang/Nützlichkeit Angebote“, „Abbau von Vorbehalten“ jeweils eine eindeutige Bejahungstendenz; bezüglich des Ziels der „Verbesserung von Politikerkontakten“ sind hingegen eher Verneinungen ersichtlich; aufgrund der geringen Fallzahlen für die Dachverbände sind diese Neigungen allerdings nicht als für die Verbände charakteristisch zu veranschlagen.
} 
Größere Bedeutung von Finanzierungs- und Planungssicherheit für jüngere MSO

An dieser Stelle ist auch die Frage aufzuwerfen, ob das Alter der MSO hinsichtlich der Bedeutsamkeit der Kooperationsziele und der diskutierten Legitimitätsrelevanz von Belang ist. Die Anwendung des Rangkorrelationskoeffizienten nach Spearman zeigt, dass den drei Zielen der Erschließung von zusätzlichen finanziellen Mitteln, des Aufbaus einer langfristigen Zusammenarbeit sowie der Verbesserung der Bekanntheit und Vernetzung der Organisation jeweils eine größere Bedeutung zukommt, je jünger eine MSO ist. ${ }^{66}$ Darin spiegelt sich der für die jüngeren Vereinigungen relevante höhere Bedarf an einer Finanzierungs- und Planungssicherheit sowie deren stärkere auf Nachhaltigkeit ausgerichtete Zukunftsplanung. Damit zeigt sich bei den jüngeren MSO anhand der drei für sie signifikant bedeutsameren Kooperationsziele (die die Faktoren 1 und 2 sowie mit dem querladenden Item beide Faktoren betreffen) paradigmatisch, dass die faktorisierten Kooperationsziele nicht separat zu denken sind. Die größere Bedeutung des querladenden Kooperationsziels (,,Verbesserung Bekanntheit/Vernetzung“) für jüngere MSO untermauert insofern die oben akzentuierte Interpretation seiner die anderen Kooperationszwecke ermöglichenden, Hebelfunktion“ zwischen unterschiedlich ausgerichteten Zielen.

Vor dem Hintergrund der obigen Erörterungen ist einerseits zu schlussfolgern, dass der NI weiter zu denken ist, da unterschiedlich ausgerichtete Kooperationsziele miteinander verknüpft sind und der NI - zumindest in quantitativen Befragungen - über Kooperationsziele schwer einzuhegen und zu operationalisieren ist. Andererseits könnte sich angesichts der Bündelung mehrerer Kooperationszwecke für den Versuch einer erkenntniserweiternden Prüfung der Theorie des NI ein größerer Stichprobenumfang sowie eine breitere, kleinteiligere inhaltliche Operationalisierung der Ziele als opportun erweisen.

\section{Innerhalb der Kooperationen wahrgenommene Schwierigkeiten}

Rund 50 Prozent der kooperierenden MSO gaben an, innerhalb der Zusammenarbeit mit anderen Organisationen mit Schwierigkeiten konfrontiert zu sein. Darunter befinden sich jeweils circa 49 Prozent der nicht-religiösen und religiösen MSO.

Mit 9 Items wurden potenzielle Barrieren vorgegeben. Die Zustimmungswerte zeigen, dass in erster Linie ein zu hoher, komplizierter Verwaltungsaufwand

${ }^{66}$ Erschließung finanzielle Mittel: $r h o=-0,15^{*}$ - Aufbau langfristiger Zusammenarbeit: $r h o=-0,18^{*}$ — Verbesserung Bekanntheit/Vernetzung: $r h o=-0,2 * *$ (Anmerkung: Aufgrund der inversen Codierungen der ordinalskalierten Variablen zeigt ein negatives Vorzeichen von Spearman-Rho hier einen positiven Zusammenhang mit dem Organisationsalter an). 
(62\%), unterschiedliche Auffassungen über die gemeinsame Projektumsetzung $(56 \%)$ sowie eine geringe Offenheit und Vorurteile gegenüber der eigenen Organisation (54\%) als Hemmnisse wahrgenommen werden (Abb. 7.16).

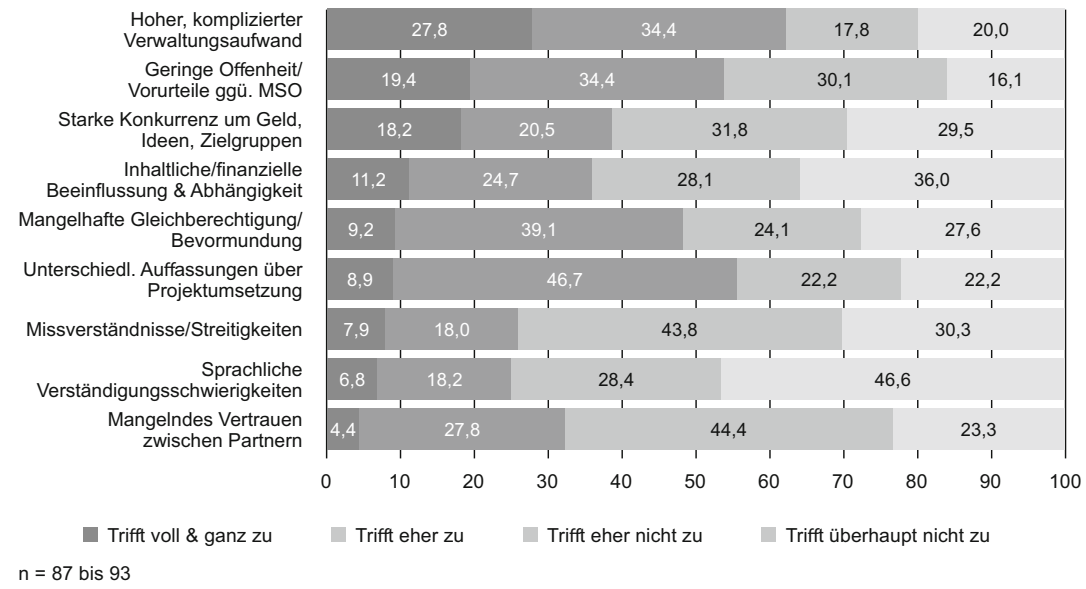

Abb.7.16 Im Rahmen der Kooperationen wahrgenommene Schwierigkeiten (in \%)

Fast die Hälfte der kooperierenden MSO (48 \%) stimmen dem Vorhandensein einer mangelhaften Gleichberechtigung zwischen oder Bevormundung durch die Partner(n) zu. Die weiteren fünf Items wurden seitens der MSO mehrheitlich nicht bekräftigt, allerdings sind hier im Antwortverhalten der religiösen und nichtreligiösen MSO zum Teil recht markante Abweichungen zu identifizieren. So wird die Existenz einer starken Konkurrenz um Geld, Ideen oder Zielgruppen zwischen den Partnern (39\%) weitaus häufiger für die säkularen MSO bejaht (MO: $49 \%$ vs. RMO: $17 \%$ ). Von diesen wird in einem stärkeren Ausmaß auch eine von außen einwirkende inhaltliche oder finanzielle Beeinflussung und Abhängigkeit ausgemacht (MO: $46 \%$ vs. RMO: $14 \%$, alle MSO: $36 \%$ ).

Hinsichtlich der Registrierung eines mangelhaften Vertrauens zwischen den Kooperationspartnern (32\%) lassen sich keine größeren Unterschiede zwischen (nicht-)religiösen MSO feststellen. Missverständnisse und Streitigkeiten (26\%) sowie sprachliche Verständigungsprobleme $(25 \%)$ stellen für jeweils circa ein Viertel der kooperierenden Organisationen Barrieren dar. 


\section{- Statistische Tests und Ergebnisdiskussion}

Anhand einer Explorativen Faktorenanalyse (EFA) lassen sich drei Faktoren identifizieren (Tab. 7.27), die sich inhaltlich wie folgt beschreiben lassen:

1) Wettbewerbsbasierte, bürokratisch-operative Hürden, die selbstbestimmtem Handeln entgegenstehen;

2) Mangelhafte Überzeugung von der (Zu-)Verlässigkeit, Vertrauens- sowie Ebenbürtigkeit der Partner in Hinblick auf Kompetenzverteilungen und Zuständigkeitsbereiche;

3) Barrieren beim Versuch des verbalen Informationsaustausches und (daraus ggf. resultierender) falscher Auslegungen von Aussagen oder Handlungen sowie Meinungsverschiedenheiten.

Tab. 7.27 Rotierte Faktorladungsmatrix zu wahrgenommenen Schwierigkeiten innerhalb der Kooperationen

\begin{tabular}{|c|c|c|c|}
\hline & \multicolumn{3}{|c|}{ Faktoren } \\
\hline & 1 & 2 & 3 \\
\hline Starke Konkurrenz um Geld, Ideen oder Zielgruppen & ,877 & , 192 &,- 023 \\
\hline $\begin{array}{l}\text { Inhaltliche und finanzielle Beeinflussung und Abhängigkeit von } \\
\text { außen }\end{array}$ & ,850 &,- 023 & ,151 \\
\hline Hoher, komplizierter Verwaltungsaufwand & ,736 & ,218 &,- 166 \\
\hline Geringe Offenheit/Vorurteile gegenüber unserer Organisation &,- 080 & ,900 & 014 \\
\hline Mangelndes Vertrauen zwischen den Kooperationspartnern & ,437 &, 654 & ,249 \\
\hline Mangelhafte Gleichberechtigung/Bevormundung durch die Partner & ,408 &, 589 & ,303 \\
\hline Unterschiedliche Auffassungen über die Umsetzung des Projektes* & 348 & ,496 & ,423 \\
\hline Sprachliche Verständigungsschwierigkeiten &, 032 &, 060 & ,901 \\
\hline Missverständnisse/Streitigkeiten &,- 097 & ,230 & ,850 \\
\hline
\end{tabular}

Extraktionsmethode: Hauptkomponentenanalyse. Fehlende Werte: paarweiser Fallausschluss. Rotationsmethode: Varimax mit Kaiser-Normalisierung. Maß der Stichprobeneignung nach Kaiser-Meyer-Olkin (KMO-Test): 0,677. Eigenwerte $>1,0 ;$ Faktorladung $\geq+/-0,5 ; \mathrm{n}=87-93$.

* Aufgrund der Ladung unter 0,5 darf dieses Item bei der Beschreibung des Faktors streng genommen nicht explizit berücksichtigt werden. Bei einem listenweisen Fallausschluss der fehlenden Werte, lädt es mit einem Wert über 0,5 auf den betreffenden Faktor und wird diesem für seine inhaltliche Beschreibung eingefügt. 
Größere Konkurrenzsituation und Abhängigkeitsverhältnisse für säkulare MSO

Die Kalkulation des U-Tests ermittelt für die Barrieren einer wahrgenommenen Konkurrenzsituation sowie einer inhaltlichen bzw. finanziellen Abhängigkeit sowie Einflussnahme von außen für die beiden Gruppen der religiösen und säkularen MSO jeweils signifikante Unterschiede: Beiden Aspekten kommt für die nicht-religiösen Vereinigungen jeweils eine größere Bedeutung zu. ${ }^{67}$

Diese Ergebnisse sollten auf die größere Anzahl und Vielfalt der seitens der nicht-religiösen Vereinigungen gewählten Kooperationspartner zurückzuführen sein; diese operieren auf unterschiedlichen gesellschaftspolitischen und administrativen Ebenen. Im Buhlen um Klientel und Fördermittel kann die Zusammenarbeit unter spezifischen Umständen wettbewerbsorientierter und spannungsreicher ausfallen. Durch bestehende Abhängigkeiten (von geldgebenden Einrichtungen) sollten seitens der säkularen MSO auch eine sie unzufrieden stimmende Unmündigkeit oder fehlende Autonomie auszumachen sein.

Die Probleme, denen am häufigsten zugestimmt wurde, betreffen bürokratische, administrative Hürden und disparate Standpunkte hinsichtlich der Projektrealisierung, die auf im Vorfeld nicht oder unzureichend erfolgte Klärungen und Absprachen zurückzuführen sein könnten. Zudem mangelt es im Rahmen einiger Kooperationen an Aufgeschlossenheit und Unvoreingenommenheit zwischen den Beteiligten. Darüber hinaus scheinen zum Teil asymmetrische Einflussnahmen und damit keine Zusammenarbeit auf der vielbeschworenen „Augenhöhe“ zu bestehen. Die Existenz dieser Barrieren ist nicht unbekannt (I. 2.2; u. a. BMFSFJ 2017: 211-213); sie verhindern stabile Kooperationen und sollten daher gezielt abgebaut werden (s. dazu auch 7.3, Fazit).

\subsubsection{Einschätzung der eigenen Arbeit und Situation}

Divergierende und differenzierte Wahrnehmungen durch Funktionsträger

Mit einer letzten Itembatterie im Fragebogen sollten seitens der MSO wahrgenommene organisations- und umweltbezogene Problemlagen und damit implizierte Handlungs- und Unterstützungsbedarfe erhoben werden. Insgesamt waren sieben Aussagen zur Arbeit und Situation der MSO vorgegeben, die anhand der bekannten Vierer-Ordinalskala einzustufen waren.

${ }^{67}$ Konkurrenzsituation $U$-Test: $U=542 ; z=-3,09 ; p=0,002 ; r=0,33 ; \operatorname{Md}_{\mathrm{S}}=3$;

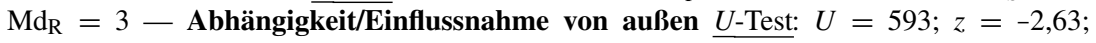
$p=0,009 ; r=0,28 ; \mathrm{Md}_{\mathrm{S}}=3 ; \mathrm{Md}_{\mathrm{R}}=4$. 
72 Prozent der befragten Funktionsträger stimmen der Aussage zu, dass die Arbeit ihrer Organisation professioneller und qualifizierter sein müsste, und 66 Prozent sind der Auffassung, dass die Tätigkeiten der MSO zu wenig öffentliche Wertschätzung und Anerkennung erfahren (Abb. 7.17).

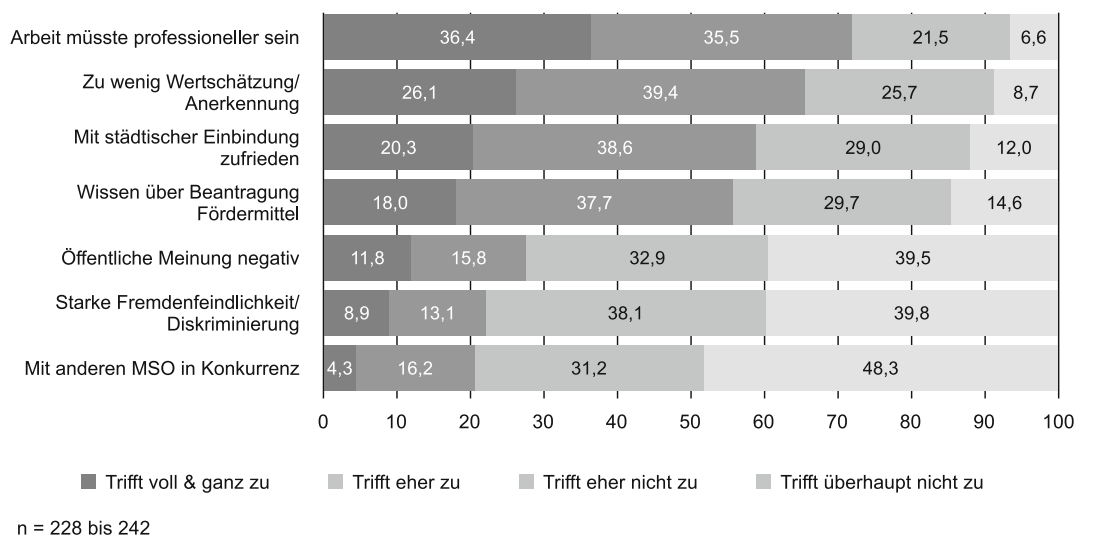

Abb. 7.17 Einschätzung der eigenen Arbeit und Situation (in \%)

Im Gegensatz zur als mangelhaft eingeschätzten Wertschätzung geben 59 Prozent der Befragten an, mit der Einbindung ihrer Organisation seitens der Stadt zufrieden zu sein und mehr als die Hälfte $(56 \%)$ bestätigt, dass in ihrer Organisation bekannt ist, wo und wie Fördermittel beantragt werden können. Die beiden Aussagen, dass ihre Organisation in einem starken Ausmaß mit Fremdenfeindlichkeit und Diskriminierung konfrontiert und dass die öffentliche Meinung über ihre Gruppe negativ ist, werden mit jeweils über 70 Prozent verneint. Bei einer näheren Betrachtung der 22 Prozent, die sich von Fremdenfeindlichkeit und Diskriminierung betroffen fühlen, zeigt sich, dass sich darunter mehrheitlich religiöse MSO befinden ( $33 \%{ }^{68}$ vs. $18 \%$ ). Zudem sind die religiösen MSO mit einem um 21 Prozentpunkte höher liegenden Wert eher der Ansicht, dass über die eigene Gruppe eine negative öffentliche Meinung vorherrscht (RMO: $43 \%$ vs. MO: $22 \%$, alle MSO: $28 \%$ ).

\footnotetext{
68 Von insgesamt 73 religiösen MSO, die die Frage nach der Wahrnehmung von Fremdenfeindlichkeit und Diskriminierung beantwortet haben, bejahen dies 15 islamische, 4 christliche, 3 jüdische (und damit alle im Sample vertretenen jüdischen Organisationen), eine alevitische und eine ezidische.
} 
Für die Mehrzahl der MSO wird zudem keine Konkurrenzsituation zu anderen MSO registriert. Unter den 21 Prozent der MSO, für die eine Konkurrenz wahrgenommen wird, befinden sind mehrheitlich nicht-religiöse MSO (MO: $25 \%$ vs. RMO: $10 \%$ ).

Die Ergebnisse zu den vier erstgenannten Items (Abb. 7.17) verweisen auf Optimierungspotenziale und implizieren Unterstützungs- und Verbesserungsbedarfe, die bislang scheinbar noch nicht in einem ausreichenden Maße ausgeschöpft wurden.

Als Patt sind die Einschätzungen zur mangelhaften Wertschätzung gegenüber der Zufriedenheit mit der städtischen Einbindung (66\% vs. $59 \%$ zu betrachten; auch hier verweisen die Prozentwerte auf erwünschte Optimierungen. Im Gesamtbild positiv zu werten ist, dass mehrheitlich keine Fremdenfeindlichkeit/Diskriminierung und mehrheitlich keine öffentlichen Fehlplatzierungen registriert werden.

\section{- Statistische Tests, Hypothesenprüfung und Ergebnisdiskussion}

Unterschiede in der Wahrnehmung von Fremdenfeindlichkeit und Konkurrenz

Berechnungen des $U$-Tests zeigen, dass die Wahrnehmung von Fremdenfeindlichkeit bzw. Diskriminierung sowie einer negativen öffentlichen Meinung bei den religiösen MSO jeweils signifikant stärker ausgeprägt ist als bei den säkularen. ${ }^{69}$ Bei letzteren fällt hingegen die empfundene Konkurrenzsituation zu anderen MSO größer aus (Tab. 7.28).

Diese Ergebnisse sind insofern nicht überraschend, als religiöse MSO und in erster Linie islamische, die auch in der Substichprobe der religiösen MSO dominant vertreten sind, mit größeren Vorbehalten und Misstrauen konfrontiert werden (Nagel 2015b: 31). Insbesondere infolge der Terroranschläge des 11. Septembers 2001 sind Personen muslimischen Glaubens verstärkt in den Fokus der (deutschen) Öffentlichkeit geraten und die Religion des Islam ist zu einem prominenten Thema in der bundesdeutschen Sicherheits- und Integrationsdebatte geworden. Islamische Religionszugehörigkeit bzw. muslimische Religiosität wird seither tendenziell mit Misstrauen und als ein Katalysator für die Etablierung ,parallelgesellschaftlicher" Strukturen betrachtet. Jüngere Studien weisen nach, dass die Haltung der Deutschen insbesondere gegenüber Muslimen mehrheitlich negativ ausfällt (Pollack 2014b: 34; s. auch Vopel/El-Menouar 2015); zudem sehen

\footnotetext{
${ }^{69}$ Die Berechnung des $U$-Tests für die beiden religiösen Gruppen „Islam“ und ,Sonstige Religionen“ (= Alevitentum, Christentum, Judentum, Ezidentum, Hinduismus) zeigt für die Merkmalsausprägungen der Wahrnehmung von Fremdenfeindlichkeit sowie einer negativen öffentlichen Meinung keine signifikanten Ergebnisse.
} 
Tab. 7.28 $U$-Test: Ergebnisse für die Wahrnehmungen der Umweltbeziehungen (Gruppeneinteilung ,religiös/nicht-religiös“)

\begin{tabular}{|c|c|c|c|}
\hline$U$-Test & $\begin{array}{l}\text { Starke Wahrnehmung } \\
\text { von Fremdenfeindlich- } \\
\text { keit/Diskriminierung }\end{array}$ & $\begin{array}{l}\text { Wahrnehmung von } \\
\text { negativer öffentlicher } \\
\text { Meinung über MSO }\end{array}$ & $\begin{array}{c}\text { Wahrnehmung von } \\
\text { Konkurrenz zu anderen } \\
\text { MSO }\end{array}$ \\
\hline$U$-Wert & 4216,5 & 3827,5 & 4864,5 \\
\hline$z$-Wert & $-3,81$ & $-3,4$ & $-2,19$ \\
\hline Median $_{\mathrm{s}}$ & 3 & 3 & 3 \\
\hline $\operatorname{Median}_{R}$ & $3^{*}$ & $3+$ & 4 \\
\hline \multirow{2}{*}{ Effektstärke $r$} & $0,25^{\star \star *}$ & $0,23^{* * *}$ & $0,14^{*}$ \\
\hline & Kleiner Effekt & Kleiner Effekt & Kleiner Effekt \\
\hline
\end{tabular}

- Anmerkung: Die Betrachtung der gemittelten Ränge bzw. Rangsummen indiziert höhere Merkmalsausprägungen für die Perzeption von Fremdenfeindlichkeit und unzutreffenden Sichtweisen unter den religiösen MSO.

viele Deutsche im Islam „etwas Fremdes, Andersartiges und Bedrohliches“ (Pollack/Müller 2013: 43; II. 3.2). Neben der realen und gefühlten Bedrohung durch islamistischen Terror können auch selektive, negative Medienberichterstattungen, konflikthafte politische Entwicklungen und Ereignisse sowie Einflussnahmen durch die Herkunftsländer (v. a. der Türkei) als zentrale Einflussfaktoren für ablehnende und abwehrende Einstellungen gegenüber dem Islam bzw. Muslimen angeführt werden. Die Auswertung zeigt aber auch, dass andere Religionszugehörige, wie Christen und Juden, mit Fremdenfeindlichkeit, ablehnenden Haltungen und Antisemitismus konfrontiert sind (vgl. auch Küpper/Zick 2019).

Die Wahrnehmungen von Diskriminierung, Fremdenfeindlichkeit und stereotypen Merkmalszuschreibungen werden als negative Rahmenbedingungen und Situationsdeutungen für die betroffenen Individuen und MSO handlungsbestimmende Folgen haben. Im Kapitel zu den Analyseperspektiven und Erklärungsansätzen des soziologischen Neoinstitutionalismus (NI) wurde die Relevanz des gesellschaftlichen Kontextes in seinen Auswirkungen auf organisationales Handeln herausgestellt (II. 4.5). Organisationen werden nicht als kontinuierlich zweckrational agierende Kollektivakteure, sondern v. a. als Produkte ihrer gesellschaftlichen Umwelt(en) betrachtet. Hochbedeutsam ist das wechselseitige Verhältnis zwischen einer Organisation und ihrer Umwelt, auf deren wahrgenommenen Erwartungen die Organisationen auf unterschiedliche Weise reagieren. Die Organisationen können Chancen, Optionen und Opportunitäten, aber auch Risiken und Bedrohungen ausmachen. Organisationale Reaktionen und Handlungsoptionen können daher variationsreich ausfallen. Annahmen eines passiven, 
gleichförmigen, lediglich reaktiven und Konformität erzeugenden organisationalen Handelns sind zu verwerfen. Neben Rückzügen sind auch offensive Vorstöße möglich.

Welche Verhaltensformen als (nicht-)konforme Response auf von außen einwirkende Erwartungshaltungen seitens der MSO realisiert werden, hängt auch von deren Einschätzung der externen Anreize ab. So kann die Kooperationsbereitschaft z. B. beeinträchtigt werden, wenn eine Organisation die Verweigerung oder Nicht-Verfügbarkeit der erwünschten, von außen zu erlangenden Ressourcen (Legitimität, Ansehen, finanzielle Unterstützung) antizipiert oder ein zu großer Vielfältigkeitsgrad von Anforderungen besteht. Auch wenn interne Interessen in einem starken Widerspruch zu den wahrgenommenen externen Forderungen stehen, wird dies den Kooperationswillen unterminieren (Oliver 1991: 160 ff.; Rosenow-Williams 2012: 455).

Im Kontext der oben dargelegten Annahmen ist argumentiert worden (II. 4.5), dass die Wahrnehmung von pejorativen Zuschreibungen und öffentlichen NegativDiskursen einflussreiche Faktoren sind, die sich auch auf die Kooperationsbereitschaft der MSO auswirken: Im Streben nach Legitimität, Status und Ressourcenzufluss werden einige gezielt die Zusammenarbeit mit anderen Organisationen suchen, während sich andere mit großer Wahrscheinlichkeit zurückziehen, sich gegenüber der Umwelt abgrenzen und Kooperationen meiden bzw. keine kooperativen Strategien verfolgen. Daran ansetzend wurde die folgende Doppelhypothese formuliert:

H5 1 : Die Wahrnehmung von Fremdenfeindlichkeit und Diskriminierung führt mit großer Wahrscheinlichkeit dazu, dass sich MSO zurückziehen und keine Kooperationen eingehen.

H5 $_{2}$ : Die Wahrnehmung von negativen öffentlichen Zuschreibungen und Diskursen bewirkt mit großer Wahrscheinlichkeit, dass MSO verstärkt Kooperationen eingehen.

Während sich $\mathbf{H 5}_{1}$ mit den vorliegenden Daten nicht bestätigen lässt, könnte sich $\mathbf{H 5}_{2}$ als zutreffend erweisen.

In Kreuztabellen zeigen sich keine Zusammenhänge zwischen der Wahrnehmung von Fremdenfeindlichkeit und Diskriminierung oder einer negativen öffentlichen Meinung sowie Nicht-Kooperationen. Unter den MSO, die kooperieren (76\%), sind die beiden Merkmale jeweils höher ausgeprägt als bei der deutlich geringeren Anzahl der befragten Vereinigungen, die nicht mit anderen Organisationen zusammenarbeiten $(24 \%)$. 
Keine negativen Auswirkungen von Fremdenfeindlichkeit und Fehlplatzierungen auf Kooperationsverhalten

Werden die kooperierenden und nicht-kooperierenden MSO als zwei Gruppen betrachtet und der $U$-Test berechnet, zeigt sich, dass für die beiden Items bei den kooperierenden Vereinigungen jeweils signifikant höhere Zustimmungsraten vorzufinden sind. ${ }^{70}$ Die bereits berichtete insgesamt gering ausgeprägte Wahrnehmung von Fremdenfeindlichkeit/Diskriminierung sowie negativen öffentlichen Ansichten ließ bereits vermuten, dass sich die beiden Phänomene nicht negativ auf die unter den MSO grundsätzlich stark ausgeprägten Kooperationen auswirken dürften. Die Ergebnisse sprechen eher dafür, dass die MSO auch bei Wahrnehmung der beiden negativen Phänomene mit anderen Einrichtungen zusammenarbeiten und dass die Perzeption von Herabsetzungen und fremdenfeindlichen Gesinnungen demnach insgesamt nicht als für Nicht-Kooperationen ursächlich betrachtet werden kann. Anhand der Testergebnisse kann jedoch nicht auf kausale Zusammenhänge im Sinne von Ursache und Wirkung vorgefundener Zustände geschlossen werden: Es kann nicht festgestellt werden, inwieweit das Kooperationsverhalten eine bewusste Reaktion auf registrierte gesellschaftliche Fehlplatzierungen darstellt und inwieweit die Phänomene tatsächlich aufeinander bezogen sind.

Allerdings springt die Prüfung der Hypothese lediglich mit der dichotomen Variablen (Kooperation: nein/ja, 0/1) zu kurz und ist wenig überzeugend. Denn es stellt sich die berechtigte Frage, ob bei der Wahrnehmung von Fremdenfeindlichkeit und einer negativen öffentlichen Meinung nicht nur der Aspekt eines überhaupt existenten Kooperationsverhaltens, sondern auch der Typus des gewählten Kooperationspartners eine Rolle spielt. Bivariate Tabellierungen der Kooperationspartnertypen mit den beiden ordinalskalierten Variablen (Fremdenfeindlichkeit; öffentliche Meinung) lassen erkennen, dass keine negativen Zusammenhänge zwischen der ohnehin geringen Zustimmung zu den beiden Items sowie der Kooperationsbereitschaft besteht. Dies zeigt sich insbesondere auch bei den drei beliebtesten Kooperationspartnern (vgl. 7.1.6): anderen MSO, ortsansässigen Vereinen sowie Ämtern in der eigenen Stadt, bei denen bei höheren Merkmalsausprägungen eine Kooperation eingegangen wird anstatt letztere zu

\footnotetext{
${ }^{70} U$-Tests: Fremdenfeindlichkeit \& Diskriminierung / Gruppe (nicht-)kooperierende MSO: $U=3347 ; z=-3,66 ; p=0,000$; koop. MSO: Md = 3; nicht-koop. MSO: $\mathrm{Md}=4$; $r=0,24-$ Negative öffentliche Meinung / Gruppe (nicht-)kooperierende MSO: $U=3321$; $z=-3,03 ; p=0,002$; koop. MSO: Md =3; nicht-koop. MSO: $\mathrm{Md}=4 ; r=0,2$.
} 
unterlassen. Auch bei den Wohlfahrtsverbänden, Integrationsagenturen und Kirchen, mit denen rund 35 bis 39 Prozent der MSO zusammenarbeiten, zeigt sich, dass bei der Empfindung von negativen, diskriminierenden Einstellungen gegenüber der eigenen Gruppe mit den Einrichtungen kooperiert wird. Die Durchführung des $U$-Tests zeigt für die Kooperationstypen MSO und Kirche, dass mit diesen scheinbar gerade bei Konfrontation mit xenophoben Haltungen zusammengearbeitet wird: Die Separation der befragten kooperierenden MSO nach mit MSO oder Kirchen (nicht-)kooperierenden Gruppen zeigt für die kooperierende Gruppe jeweils höhere Merkmalsausprägungen mit einer signifikanten kleinen Effektstärke $^{71}$. Insofern könnte angenommen werden, dass sich die betroffenen MSO die Einrichtungen als Verbündete suchen, von denen keine Diskriminierung ausgeht.

Eine weitere Prüfung von in den Hypothesen implizierten möglichen Zusammenhängen zwischen der Wahrnehmung von Fremdenfeindlichkeit bzw. Diskriminierung oder einer negativen öffentlichen Meinung mit der Anzahl der Kooperationspartnertypen zeigt keine signifikanten Zusammenhänge. ${ }^{72}$

Die Tatsache, dass bei negativen Zuschreibungsprozessen kooperiert wird, negiert die Annahme von allgemeinen Rückzugstendenzen aufgrund wahrgenommener Abwertungen der eigenen Gruppe. Die beiden Phänomene „Diskriminierung/Fremdenfeindlichkeit“ und ,negative Meinungen“ resultieren nicht - wie mit der Doppelhypothese konstruiert - in unterschiedlichem Kooperationsverhalten, sondern sie besitzen den gleichen Stellenwert. Wie oben und im Zusammenhang mit der Formulierung der Hypothese dargelegt wurde (II. 4.5), kann im Rahmen des Erhebungsdesigns nicht gesichert ermittelt werden, inwieweit seitens der MSO als Diskriminierung und gesellschaftliche Fehlplatzierungen gewertete Fremdzuschreibungen konkrete Auslöser für spezifische Strategien oder Taktiken des Kooperierens darstellen. Die Bearbeitung von Diskriminierungserfahrungen und Negativ-Diskursen muss sich nicht zwangsläufig im Kooperationsverhalten spiegeln, wobei dieses angesichts der Ergebnisse nicht per se ausgeschlossen werden kann. Denn die signifikanten Testergebnisse für die Kooperationspartner MSO und Kirche geben einen Hinweis auf stärker ausgeprägte Kooperationen in

${ }^{71} U$-Test für den Kooperationspartner MSO: $U=3020,5 ; z=-2,02 ; p=0,044$; Koop. mit

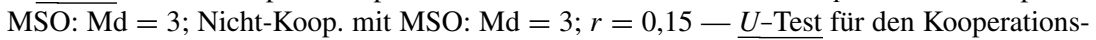
partner Kirche: $U=3066 ; z=-2,38 ; p=0,017$; Koop. mit Kirche: Md = 3; Nicht-Koop. mit Kirche: $\mathrm{Md}=3 ; r=0,18$.

${ }^{72}$ Rangkorrelationskoeffizient nach Spearman: Fremdenfeindlichkeit \& Diskriminierung / Kooperationspartneranzahl: $r h o=-0,08, p=0,304=$ n. s., $n=183$ - Negative öffentliche Meinung / Kooperationspartneranzahl: $r h o=0,09, p=0,259=$ n. s., $n=177$. 
Anbetracht wahrgenommener xenophober Haltungen und negativer öffentlicher Meinungen.

Darüber hinaus wurde im Kapitel zum NI thematisiert, dass Wettbewerb und Konkurrenz wesentliche Einflussfaktoren darstellen, die die Interaktionen und Handlungen der MSO innerhalb des organisationalen Felds wesentlich mitbestimmen (Hunger/Metzger 2011, 2013a; Rosenow-Williams 2012; Halm et al. 2012a; Olgun 2015; Blätte 2014). In unterschiedlichen Ausmaßen besteht ein Wettstreit um finanzielle Ressourcen, Mitglieder, Zielgruppen, politische Legitimität, die Unterstützung durch Öffentlichkeit und Mitglieder wie auch ein Wettbewerb zwischen den islamischen Verbänden sowie religiösen und nicht-religiösen MSO im Besonderen (u. a. Rosenow-Williams 2012: 231, 437 ff., 469). Vor dem Hintergrund der Annahme von im organisationalen Feld bestehenden diversen Konkurrenzverhältnissen wurde folgende Hypothese aufgestellt:

H7: Wettbewerb belebt das Kooperationsgeschehen: MSO, die andere MSO als Konkurrenten betrachten, arbeiten nicht mit anderen MSO, sondern v. a. mit städtischen und staatlichen Behörden zusammen.

Kooperation von miteinander konkurrierenden MSO

Die Hypothese kann nicht bestätigt werden. Es lassen sich keine signifikanten Zusammenhänge oder Verteilungen zwischen der Registrierung einer Konkurrenzsituation unter den MSO und der Zusammenarbeit mit städtischen oder staatlichen Organisationen identifizieren. Hingegen scheinen allerdings Wohlfahrtsverbände wichtige Kooperationspartner bei wahrgenommenen Konkurrenzsituationen mit anderen MSO zu sein; für die Wohlfahrtsverbände ermittelt der $U$-Test signifikante schwache Effekte. Paradoxerweise - und diametral zur hypothetischen Annahme - ist unter den MSO, die mit anderen migrantischen Vereinigungen zusammenarbeiten, das Merkmal des In-Konkurrenz-Stehens signifikant höher ausgeprägt als unter den mit anderen MSO nicht-kooperierenden. ${ }^{73}$ - Wie sind diese Ergebnisse zu deuten?

\footnotetext{
${ }^{73}$ Für die Berechnung des $\underline{U \text {-Tests }}$ (Konkurrenzsituation/Kooperation) wurden die MSO jeweils als zwei mit einem spezifischen Organisationstypus (nicht)-kooperierende Gruppen betrachtet. Das Ergebnis für den Kooperationspartner Wohlfahrtsverband (WV): $U=2983$; $z=-2,97 ; p=0,003$; Koop. mit WV: Md = 3; Nicht-Koop. mit WV: Md = 4; $r=0,22$ - $\underline{U}$-Test für den Kooperationspartner MSO: $U=2709,5 ; z=-2,88 ; p=0,004$; Koop. MSO: $\mathrm{Md}=3$; Nicht-Koop. MSO: $\mathrm{Md}=4 ; r=0,21$.
} 
Das Phänomen der erhöhten und bevorzugten Kooperation mit Wohlfahrtsverbänden - bei einer wahrgenommenen Konkurrenzsituation mit anderen MSO - ist auch im Zusammenhang mit der Beantwortung der Frage nach der Mitgliedschaft in Dachverbänden zu erklären (7.1.1). Hier wurde am häufigsten die Mitgliedschaft im Paritätischen Wohlfahrtsverband angeführt (27 \%; $\mathrm{n}=125)$. Zudem kommt den Spitzenverbänden der Freien Wohlfahrtspflege und insbesondere dem Paritätischen Wohlfahrtsverband - historisch bedingt - bei der Integrationsarbeit sowie Beratung und Förderung von MSO im Bundesland NRW eine zentrale Funktion zu. Der Paritätische hat viele MSO, in seine Strukturen integriert, wovon die Arbeit der Organisationen deutlich profitiert" (Halm et al. 2020: 122).

Im Paritätischen NRW existiert seit dem Jahr 2000 eine durch das Integrationsministerium geförderte Fachberatung für MSO. Deren Aufgaben sind die Qualifizierung, Vernetzung und Unterstützung der Arbeit von MSO wie auch die Hilfeleistung bei der Gründung von Selbstorganisationen Neuzugewanderter. ${ }^{74}$ Darüber hinaus übernehmen die Wohlfahrtsverbände viele Aufgaben in der sozialraumorientierten und Antidiskriminierungsarbeit. Ihre Zuständigkeit für Angelegenheiten von Menschen mit einer Zuwanderungsgeschichte hängt auch mit ihrer Übernahme der Trägerschaft der seit 2007 politischerseits geförderten Integrationsagenturen mit dort beschäftigten Integrationsfachkräften zusammen. ${ }^{75}$ Insofern erscheint die Präferenz des Kooperationstypus Wohlfahrtsverband sehr nachvollziehbar und plausibel (vgl. auch Friedrichs et al. 2020: 54 f., 58 f.).

Die zwischen unterschiedlichen MSO potenziell bestehenden wettbewerbsintensiven Beziehungen und daraus resultierende Verhaltensweisen sind im Gegensatz zum interreligiösen Dialog der Gegenwart noch wenig erforscht. Der Widerspruch, dass untereinander in Konkurrenz stehende MSO miteinander kooperieren, lässt sich jedoch auflösen, wenn man von einem Kooperationswettbewerb (engl. „co-opetition“) ausgeht (Jansen et al. 2000). In unabwendbaren Wettbewerbssituationen um knappe Ressourcen kann Konkurrenz in der Tat das Geschäft und die Kooperationsbereitschaft beleben und das Phänomen auftreten, dass sich Konkurrenten verbünden, um über Kartellbildung oder als „,Beutegemeinschaft““ (vgl. Friedrichs et al. 2020: 61) in der Bewerbung um Fördermittel Interessen zu bündeln, gemeinsam Vorteile und Erfolge zu erzielen und Außenseiterpositionen, die aus Diskriminierungserfahrungen resultieren können, zu

\footnotetext{
${ }^{74}$ https://www.paritaet-nrw.org/soziale-arbeit/themen/migration/migrantenselbstorganisat ionen/(letzter Abruf: 03.04.2021).

75 http://www.kfi.nrw.de/Foerderprogramme/Integrationsagenturen/index.php (letzter Abruf: 03.04.2021).
} 
vermeiden. Eine solche Konstellation kann sich dann - auch für die betreffenden MSO in NRW - als Win-win-Situation erweisen.

\subsection{MSO zwischen bonding und bridging}

Ein wesentliches Erkenntnisinteresse dieser Arbeit besteht darin, die Bedeutung von kollektiven - insbesondere religiösen - Zugehörigkeiten für zivilgesellschaftliche Performanzen migrantischer Selbstorganisationen zu ermitteln: Sind religiöse Bindungen in Hinblick auf zivilgesellschaftliche Performanzen eher als Katalysatoren oder eher als Barrieren einzustufen? Präziser gefragt: Welche Religionstraditionen erschweren und welche begünstigen zivilgesellschaftliche Beteiligung? Und: Inwieweit lassen sich Abgrenzungs- und Exklusionsmechanismen feststellen? Diese Fragen sind nicht pauschal zu beantworten (II. 4.2.5), ihnen soll in diesem Kapitel empirisch nachgegangen werden. Sie sind unmittelbar verknüpft mit dem Konzept von Sozialkapital, und zwar insbesondere in Hinblick auf die Differenzierung zwischen „Bridging“- und „Bonding“-Ausprägungen, die in der sozialwissenschaftlichen Theoriebildung ein arbeitshypothetisch anerkanntes Unterscheidungskriterium darstellt (II. 4.2.3). Dieses wird für die Einschätzung sozialintegrativer Wirkrichtungen von Netzwerken bzw. Vereinigungen und deren Tätigkeiten insbesondere auch im Kontext von Religion und ethnischer Pluralisierung herangezogen. Robert Putnam (2000: 22 f.) prägte die Definition eines inklusiven bzw. zwischen verschiedenen Menschengruppen brückenbauenden Sozialkapitals (,,bridging social capital“) und eines demgegenüber exklusiven oder „,bonding social capital“, das ausschließlich bestehende interne Bindungen in sich stark ähnelnden, merkmalsgleichen Gruppierungen festigt. Letztlich geht es damit um die Art der Gruppenbezogenheit und damit einhergehend um die Frage, ob Tätigkeiten eher auf Integration und Teilhabe ausgerichtet sind oder ob sie sich wesentlich auf die Förderung des Zusammenhalts der eigenen Gruppe beziehen.

Eine theoretische - aber bislang empirisch nicht validierte - Annahme ist, dass brückenbildendes Sozialkapital in sozial heterogen zusammengesetzten Vereinigungen entsteht, während in sozial homogenen Gemeinschaften eher bindendes Sozialkapital produziert wird. Wenn Menschen unterschiedlicher sozialer und kultureller Herkunft zusammenkommen und gemeinsam aktiv sind, könnten Vorurteile abgebaut, Kooperationsbereitschaft eingeübt, tolerante Einstellungen langfristig geprägt, Konflikt- und Trennlinien sowie Anzeichen von Isolierung überwunden werden (Putnam 2000: 311 ff., passim; Offe/Fuchs 2001: 417 ff.). In homogenen Vereinigungen, die sich wesentlich auf die Förderung des Zusammenhalts der eigenen Gruppe konzentrieren, unterblieben diese positiven Effekte 
hingegen. Somit werden herkunfts- und wertheterogenen Vereinigungen, die für unterschiedliche Gesellschaftsmitglieder offen sind, die sich mit ihrer Umwelt austauschen und mit anderen Organisationen zusammenarbeiten, positivere sozialintegrative und demokratieförderliche Wirkungen beigemessen. ${ }^{76}$

Bonding-Ausrichtungen sind insbesondere dann als problematisch und desintegrativ einzustufen, wenn Andockmöglichkeiten zur Aufnahmegesellschaft grundsätzlich in Abrede gestellt oder verweigert werden, um eigene Ideologien durchzusetzen und damit die Residenzkultur grundsätzlich zu bekämpfen. Im Fokus von Öffentlichkeit, Medien und Politik stehen dabei „die Muslime“ bzw. „,der Islam“, der u. a. mit Gewaltbereitschaft, mit ideologisch-fundamentalistischen und zum politischen Extremismus neigenden Positionen assoziiert wird, was in der Bevölkerung zu Abwehrhaltungen, Verunsicherung und diffusen Ängsten führt. Auf einem weniger devianten und extremen Niveau spielen aktuell - insbesondere in Nordrhein-Westfalen - erstarkende nationalistische Orientierungen von Türkeistämmigen eine Rolle, und zwar sowohl auf der Ebene von Personen als auch von Organisationen (Sauer 2018). Sie sind politisch brisant, weil die Unterstützung des türkischen Präsidenten Recep Tayyip Erdoğan und seines im Juli 2018 eingeführten Präsidialsystems sowie der sukzessive verschärften Einschränkung von demokratischen, rechtsstaatlichen Prinzipen einem Engagement entgegenstehen, das auf Integration und Teilhabe in Deutschland ausgerichtet ist. Insofern bleibt insbesondere der Islam türkischer Provenienz und die Orientierungen und Verhaltensweisen von Funktionären und Mitgliedern der islamischen Verbände ein öffentliches und politisches Dauerthema (I. 2.2). Inwieweit sich solche Orientierungen in den erhobenen Daten spiegeln, soll die in diesem Kapitel vollzogene Auswertung eruieren.

An dieser Stelle ist jedoch noch einmal herauszustellen, dass bridging und bonding nicht einfach schematisch mit integrativen oder desintegrativen Leistungen und Wirkrichtungen der MSO gleichzusetzen sind. Es wurde dargelegt (II. 4.2.3), dass auch der Bonding-Ausprägungsform positive Wirkungen zugesprochen werden. Denn auch binnenorientierte Unterstützungsleistungen und Solidarität können sozialintegrative Effekte generieren bzw. werden auch als Voraussetzung für letztere betrachtet. Eine starke Exklusivität der Zielgruppe (,Die MSO nur für die Mitglieder') muss keinesfalls mit desintegrativen und

\footnotetext{
${ }^{76}$ Die konzeptuelle Differenzierung von bonding und bridging im Kontext der theoretisch geltend gemachten Relevanz einer heterogenen oder homogenen Mitgliederzusammensetzung impliziert die Bedeutsamkeit von Personenmerkmalen der Mitglieder, die sich in einer Vereinigung aggregieren. Relevant sind sozioökonomische Aspekte (Alter, Geschlecht, Bildungsniveau, Erwerbsstatus, Einkommen) sowie ethnische und konfessionelle Zugehörigkeiten (s. auch Putnam 2000: 22 f.; Offe/Fuchs 2001: 504).
} 
abschließenden Tendenzen einhergehen, wenn sich z. B. intensiv um die Verbesserung der Bildungsteilhabe der Mitglieder gekümmert wird. Auch selbstorganisierte Zusammenschlüsse von Menschen, die sich in ähnlichen belastenden Lebenssituationen befinden und diese gemeinsam bewältigen bzw. mit ihnen umgehen lernen wollen, können exklusiv, aber mittel- und langfristig auch brückenbildend sein, da die soziale Affiliation, das Erleben von Selbstbestimmtheit und allgemeiner Kompetenzzuwachs in der Gruppe auch zur Vertretung ihrer Interessen nach außen befähigen und damit auch die soziale und rechtliche Integration fördern können (Uslucan 2015; Baumann 2004, 2016). Solche nicht prima vista zu kalkulierenden Wirkungen einer ursprünglich auf den ,Innenbereich' zielenden Gruppendynamik sprechen für eine wert- und vorurteilsfreie(re) Betrachtung von Bonding-Tendenzen.

Die in diesem Kapitel dargelegte schrittweise Analyse greift die gesellschaftspolitische Debatte auf und knüpft zugleich an Putnams zentraler Prämisse an, dass die konzeptuelle Unterscheidung von Bonding- und Bridging-Merkmalen insofern eine konstruierte ist, als Vereine, Verbände oder Gemeinschaften und deren Mitglieder sich nicht einfach und eindeutig der einen oder anderen Ausprägungsform zuordnen lassen. Vielmehr kann angenommen werden, dass beide Aspekte in der Realität gleichzeitig auftreten und sich in der Summe als Tendenzen entsprechend einem „Mehr oder Weniger“ realisieren (Putnam 2000: 23).

Für die statistische Auswertung in den Blick genommen werden vergleichende Gegenüberstellungen von Bridging- und Bonding-Ausprägungen für alle befragten MSO sowie die nicht-religiösen im Kontrast zu den religiösen, wobei auch unterschiedliche Religionstraditionen untereinander verglichen werden. Das Ziel ist es, anhand der Zusammenstellung analyserelevanter Variablen Regelmäßigkeiten bzw. Muster zu identifizieren und auf Basis unterschiedlicher, je spezifischer Merkmalsausprägungen Bonding- und Bridging-Typen zu ermitteln.

Ein wesentliches Anliegen ist es, im Rahmen der Auswertung folgende Fragen zu beantworten (II. 4.2.5):

- Haben Binnenorientierung, fehlende Kontakte zu und fehlende Offenheit gegenüber der Umwelt mit Religion zu tun und lassen sich zwischen den Religionen Unterschiede aufdecken?

- Unter welchen Bedingungen bleiben die MSO unter sich?

- Lassen sich finanzielle Mängel, personelle Ressourcenausstattungen oder das Alter der Organisationen als Erklärungsfaktoren für auf die organisationale Binnenwelt gerichtete Performanzen identifizieren?

- Sind auszumachende Bonding-Tendenzen tatsächlich auf die theoretisch als ursächlich angenommene homogene Mitglieder- und Engagiertenstruktur zurïckzuführen (Personengruppen, Konfessionszugehörigkeit, Herkunftsländer)? 
- Inwieweit ist die Wahrnehmung von Fremdenfeindlichkeit, gesellschaftlichen Fehlplatzierungen und städtischer Nicht-Einbindung seitens der MSO zu registrieren und erklärt möglicherweise auf die eigene Gruppe konzentrierte Tätigkeiten?

\section{- Zentrale Ergebnisse}

Insgesamt fördert die Auswertung folgende zentrale Befunde zutage, die der kleinteiligen Ergebnispräsentation in diesem Kapitel zur überblicksartigen Orientierung vorangestellt seien:

- Es zeigt sich ein variierendes Zusammenspiel von bonding und bridging, die keine „Entweder-Oder“-Kategorien im Sinne bipolarer Gegensätze, sondern vielmehr „Mehr oder weniger“-Tendenzen darstellen (Putnam 2000: 23). Je nach Gegenüberstellung von jeweils spezifisch ausfallenden Tätigkeiten, Zielen und Selbstbildern divergieren die jeweiligen Ausprägungsformen. Die Mehrzahl der MSO zeigt sich gegenüber der Umwelt (sehr) aufgeschlossen.

- Ein wesentliches Ergebnis der Studie lautet, dass unter Berücksichtigung verschiedener Analyseebenen und Auswertungsverfahren keine besondere Bonding-Affinität der religiösen MSO festzustellen ist. Demnach ist von einer rigorosen Einordnung von Religionen in entweder bridging oder bonding Abstand zu nehmen. Die häufigen Verdachtsäußerungen einer primären BondingOrientierung an Herkunftskulturen sowie Abschottungstendenzen religiöser - insbesondere islamischer - MSO lassen sich empirisch also nicht bestätigen. Anhand multipler Regressionsanalysen zeigt sich sogar, dass Bonding-Ziele für die nicht-islamischen Religionen gewichtiger sind.

- Wenn religionsbezogene Variablen in die Auswertung einbezogen werden, weisen die religiösen im Vergleich zu den nicht-religiösen MSO erwartungsgemäß sowohl mehr Bridging- als auch mehr Bonding-Sozialkapital auf (,,positive Teststrategie"). Bei den nicht-religiösen MSO, die sich formal keiner Religionsgemeinschaft zuordnen, verhält es sich - wenig überraschend - umgekehrt: Für sie sind religiös konnotierte Aktivitäten und Organisationsziele zumeist, aber nicht per se, unwichtig. Dies zeigt sich insbesondere für die Bridging-Aktivität des interreligiösen Dialogs.

- Lässt man religionsbezogene Variablen in der Auswertung komplett unberücksichtigt, balancieren sich die Unterschiede aus und die säkularen und religiösen MSO weisen - bei im Detail schwankenden Anteilen - ähnliche Kombinationen von bridging und bonding auf. Insgesamt wird damit deutlich, dass Religion bzw. der religiöse Charakter der sich einer religiösen Tradition 
zuordnenden Organisationen ein konstitutives Unterscheidungsmerkmal ist, das deren Bridging-Leistung insgesamt nicht relativiert und (zivil-)gesellschaftlicher Produktivität nicht entgegensteht. Folglich kommen den religiösen Eigenschaften eigenständige Effekte zu, die mit anderen Bonding- und v. a. auch Bridging-Ausprägungen kompatibel sind bzw. letztere nicht abschwächen.

- Ein entscheidender Befund lautet, dass sich bei Gegenüberstellung (Spiegelung) von Variablen, die sich jeweils als Indikatoren für bridging und bonding eignen, zwischen den betrachteten Konfessionen keine großen Diskrepanzen zeigen. Innerhalb der in der Analysestichprobe zahlenmäßig stärker vertretenen einzelnen Religionstraditionen (Islam, Alevitentum, Christentum) sind tendenziell Muster im Sinne regelmäßiger Varianzen von Bonding- und Bridging-Ausprägungsformen festzustellen ${ }^{77}$. Dies verweist auf die Möglichkeit verschiedenartiger Ausrichtungen von Vereinigungen bei gleicher religiöser Traditionsbindung.

- Die Ergebnisse von Regressionen zeigen, dass die Homogenitätsthese (in Bezug auf Herkunftsländer und Konfessionszugehörigkeiten) nicht als für BondingPerformanzen der MSO ursächlich zu bestätigen ist. Des Weiteren ist die personelle Ausstattung mit Engagierten sowie Hauptamtlichen weder in Hinblick auf Bridging- noch Bonding-Aktivitäten der MSO als eine signifikante Einflussgröße einzustufen.

- Insbesondere MSO, die religiöse Aktivitäten ausführen, sind in einem signifikanten Ausmaß mit mehr Fremdenfeindlichkeit konfrontiert. Parallel zutage tretende positive Effekte der Zufriedenheit mit der städtischen Einbindung erscheinen zunächst paradox und können dahingehend interpretiert werden, dass die Ausübung religiöser Aktivitäten und eine positiv wahrgenommene städtische Einbindung gut miteinander kompatibel sind.

Die vorangestellten wesentlichen Ergebnisse werden in den einzelnen Abschnitten dieses Kapitels (7.2.2 bis 7.2.5) anhand separater Analyseschritte sukzessive ermittelt und anhand von Abbildungen und Tabellen optisch visualisiert. Im Folgenden sind für den Nachvollzug jedoch zunächst die einzelnen Schritte des Auswertungsverfahrens darzustellen.

\footnotetext{
${ }^{77}$ Die ist insbesondere für die in der Substichprobe der religiösen Vereinigungen am stärksten vertretenen islamischen MSO festzustellen.
} 


\subsubsection{Auswertungsschritte und Analysedimensionen}

Die Auswertung basiert auf drei Analyseverfahren. Zunächst werden das Zusammenspiel und Häufigkeitsverteilungen von Bonding/Bridging-Performanzen in Hinblick auf Aktivitäten, Organisationsziele und Selbstverständnisse anhand ausgewählter Variablen-Spiegelungen deskriptiv-statistisch dargelegt, die Ergebnisse beschrieben und im Einzelnen diskutiert (7.2.2).

Um ein Gesamtbild zu den jeweiligen Ausrichtungen sowie diesbezüglichen Konfigurationen der MSO zu erhalten, wird darüber hinaus die Einnahme einer „Weitwinkelperspektive“ als erforderlich erachtet. Daher wird auf Basis von nominalskalierten ,Sammel-Variablen“, in denen Antwortkombinationen pro Organisation zusammengeführt werden, neben einem deskriptiv-statistischen auch ein qualitativ ausgerichtetes Verfahren mit interpretativem Anteil eingesetzt. Mit diesem werden mehrere relevante Variablenausprägungen, die auch die Offenheit der MSO gegenüber ihrer Umwelt betreffen, gemeinsam betrachtet, ihre Kombinationen erläutert, diskutiert (7.2.3) und die MSO verschiedenen Bonding/Bridging-Typen zugeordnet (7.2.4). Im Rahmen dieser Vorgehensweise findet ebenfalls ein zweistufiger Abgleich zwischen mitgeteilten Selbstbildern, dem Aktivitätsspektrum sowie der Offenheit der MSO statt, um Widersprüchlichkeiten - Inkommensurabilitäten - in den Selbsteinschätzungen der MSO aufdecken zu können.

Diese Auswertungsschritte werden dann in einem dritten Schritt flankiert und ergänzt von statistischen multivariaten Analyseverfahren, konkret Clustersowie Regressionsanalysen. Mit den Clusteranalysen wird anhand von Algorithmen statistisch überprüft, ob in den Daten in Bezug auf Bonding- und Bridging-Performanzen homogene Gruppen, die sich von anderen absetzen, zu identifizieren sind. Mit multiplen Regressionen wird hingegen getestet, ob Zusammenhänge bestehen zwischen als abhängige Variablen modulierten Bridgingund Bonding-Skalen einerseits und aufgrund theoretischer Erwägungen herangezogener unabhängiger, erklärender Variablen („Prädiktoren“), welche die Skalenausprägungen quasi vorhersagen, andererseits (7.2.5).

In einem Fazit werden die Ergebnisse abschließend zusammengeführt, eingeordnet und diskutiert (7.2.6). 
Die einzelnen Auswertungsschritte dieses Kapitels im Überblick

7.2.2 Das Zusammenspiel von bonding und bridging

- Aktivitäten $(\rightarrow$ Zwischenfazit)

- Organisationsziele

- Selbstverständnisse

- Überprüfung von (In-)Kommensurabilitäten I

7.2.3 Aufgeschlossenheit gegenüber der Umwelt:

Mitgliedschaftsvoraussetzungen, Zielgruppen, Kooperationsverhalten

7.2.4 Quervergleich zwischen den MSO: bridging oder bonding? -

Muster und Typenbildung

- Überprüfung von (In-)Kommensurabilitäten II

7.2.5 Untersuchung von Begründungszusammenhängen und Clusteranalysen

- Deskriptives Analyseverfahren auf Basis der Typenbildung ( $\rightarrow$ Zwischenfazit)

- Clusteranalysen

- Multiple Regressionsanalysen

7.2.6 Fazit

Für den Nachvollzug der Vorgehensweise ist es wichtig zu wissen, dass für die Analyse im Fragebogen enthaltene Variablen (Items) in den Blick genommen wurden, die sich einerseits als Indikatoren für Formen und Aktivitäten des Brückenschlags zu anderen Gruppen (bridging) eignen; andererseits wurden Variablen selektiert, die als Indizien für Selbstbezüglichkeit, ein Unter-SichBleiben und Exklusivität (bonding) der MSO und ihr Handeln fungieren können. Insgesamt beziehen sich die selektierten Bonding/Bridging-Variablen auf die Dimensionen Aktivitäten, Organisationsziele und Selbstverständnisse. Die ausgewählten Variablen werden einander gegenübergestellt, wobei die Spiegelung nicht immer inhaltlich deckungsgleich ausfällt. Die Tab. 7.29 umfasst einen Überblick zu den jeweiligen Spiegelungen.

$\mathrm{Zu}$ beachten ist, dass Bonding- und Bridging-Aspekte immer spezifisch geartet sind und in einem Fragebogen nur ausschnitthaft berücksichtigt werden können. Zudem lassen sich Tätigkeiten nicht immer eindeutig der einen oder anderen Ausprägungsform zuordnen. ${ }^{78}$ Insbesondere sind einige der Aktivitäten der MSO keiner der beiden Ausprägungsformen zu subsumieren, weil sie keine Auskunft

${ }^{78}$ Anmerkung zur Zuordnung von zwei Variablen zu bridging in Tab. 7.29: Bei der „Förderung der deutschen Sprache“ muss es sich nicht um eine Bridging-A ktivität handeln; das Item eignet sich aber gut zur Gegenüberstellung mit der Herkunftssprachenförderung; das Unterrichten oder Lernen von Deutsch impliziert einen Brückenschlag zur autochthonen Gesellschaft, denn Sprache ist eine Voraussetzung für Kommunikation und Teilhabe. 
Tab.7.29 Zuordnung einzelner Variablen zu bonding und bridging und deren Spiegelung

\begin{tabular}{|c|c|c|}
\hline $\begin{array}{l}\quad \text { Bonding } \\
(\rightarrow \text { Selbstbezogenheit/Exklusivität } \\
\text { einer Gruppe })\end{array}$ & & $\begin{array}{c}\text { Bridging } \\
(\rightarrow \text { Brückenschlag zu anderen Gruppen/ } \\
\text { Mehrheitsgesellschaft; Nicht-Migranten) }\end{array}$ \\
\hline \multicolumn{3}{|c|}{ Spiegelung von jeweils drei Aktivitäten } \\
\hline $\begin{array}{l}\text { - Heimatpflege (Herkunftsländer) } \\
(49 \% ; n=221)^{\star}\end{array}$ & versus & $\begin{array}{l}\text { Stadtteilarbeit } \\
(56 \% ; n=224)\end{array}$ \\
\hline $\begin{array}{l}\text { - Förderung der Herkunftssprache } \\
(59 \% ; n=235)\end{array}$ & versus & $\begin{array}{l}\text { - Förderung der deutschen Sprache } \\
(75 \% ; n=232)\end{array}$ \\
\hline $\begin{array}{l}\text { - Religiöse Bildung/Erziehung } \\
(42 \% ; n=224)\end{array}$ & versus & $\begin{array}{l}\text { - Interreligiöser Dialog } \\
(47 \% ; n=225)\end{array}$ \\
\hline \multicolumn{3}{|c|}{ Alternative Spiegelungen von Aktivitäten } \\
\hline $\begin{array}{l}\text { - Heimatpflege (Herkunftsländer) } \\
(49 \% ; n=221) \\
\text { - Heimatpflege } \\
\text { - Heimatpflege }\end{array}$ & versus & $\begin{array}{l}\text { - Hilfsdienste speziell für Geflüchtete } \\
(48 \% ; n=226) \\
\text { - Internationale Arbeit }(45 \% ; n=228) \\
\text { - Arbeitsmarktzugang }(44 \% ; n=228)\end{array}$ \\
\hline \multicolumn{3}{|c|}{ Spiegelung von jeweils zwei Organisationszielen } \\
\hline Bonding & & Bridging \\
\hline $\begin{array}{l}\text { Die eigene Herkunftssprache und -kultur } \\
\text { in Deutschland aufrechterhalten und } \\
\text { pflegen }(69 \% ; n=237)\end{array}$ & versus & $\begin{array}{l}\text { - Etwas für das allgemeine Wohl in } \\
\text { Deutschland tun }(89 \% ; n=242)\end{array}$ \\
\hline $\begin{array}{l}\text { - Unterstützung von im Herkunftsland } \\
\text { vertretenen politischen Positionen in } \\
\text { Deutschland }(9 \% ; n=226)\end{array}$ & versus & $\begin{array}{l}\text { Parteipolitische Positionen in Deutsch- } \\
\text { land mitgestalten/beeinflussen ( } 17 \% \text {; } \\
n=228)\end{array}$ \\
\hline
\end{tabular}

\section{Separate Betrachtung der Kombination von drei weiteren Bonding-Zielen}

- Die Lebenssituation und Teilhabe von

Zugewanderten/Landsleuten in Deutsch-

land verbessern $(87 \% ; n=245)$

- Eigene Interessen vertreten, sichtbar machen $(65 \% ; n=231)$

- Den religiösen Glauben gemeinsam praktizieren $(40 \% ; n=233)$

\section{Spiegelung von Selbstverständnissen}

\section{Bonding}

Bridging

\footnotetext{
- Selbsthilfeorganisation $(55 \% ; n=227) \quad$ versus - Brückenbauer und Kulturdolmetscher $(85 \% ; n=235)$

- Integrationspromotor $(82 \% ; n=232)$
}

* Zustimmungsraten in gültigen Prozentwerten nach Komprimierung der ursprünglichen Vierer- in eine Zweier-Skala: „Trifft voll und ganz zu/Trifft eher zu“ und „Trifft eher nicht zu/Trifft überhaupt nicht zu“

Auch bei den „Hilfsdiensten speziell für Geflüchtete“ muss es sich nicht zwangsläufig um eine Bridging-Aktivität handeln. Über die Gruppenbezogenheit ist konkret keine Aussage 
über binnen- und außenorientierte Gruppenbezüge geben. Konkret betrifft dies die Kinder- und Jugend-, Mädchen- und Frauen-, Familien- oder Senioren- sowie die allgemeine Bildungsarbeit. Die fünf genannten Tätigkeiten sind als sozialarbeiterische bzw. erzieherische Dienste und daher als von gesellschaftlichem Nutzen mit potenziell brückenschlagenden Effekten einzustufen; sie werden im Rahmen der Bonding/Bridging-Auswertung kontinuierlich gesondert ausgewiesen und in die Interpretation einbezogen.

Darüber hinaus ist zu bedenken, dass Bonding/Bridging-Ausrichtungen jeweils auf MSO mit bestimmten Orientierungen (z. B. politischen, religiösen) zutreffen werden. Daher werden im nächsten Abschnitt (7.2.2) Spiegelungen mehrerer unterschiedlicher Items (bzw. Subdimensionen) in den Blick genommen, diese miteinander verglichen, allgemeine Tendenzen beschrieben und Muster identifiziert: Für welche MSO sind beide Ausprägungsformen - bonding und bridging - relevant? Für welche nur eine? Gibt es auch Organisationen, für die beides weniger oder gar nicht wichtig ist? ${ }^{79}$

Dieses Vorgehen ist auch erforderlich, um eine positive Teststrategie im Sinne einer einseitigen Suche nach die theoretischen Annahmen bestätigenden Informationen zu vermeiden. Relevant wird dies etwa in Bezug auf die als bonding kategorisierten Items der religiösen Bildung/Erziehung und der religiösen Brauchtumspflege, da sie die Bezogenheit auf eine bestimmte religiöse Gemeinschaft implizieren. Sie sind damit aber zuvorderst für die religiösen MSO relevant, sodass theoretische Annahmen zum Bonding-Charakter von religiösen MSO nur bewahrheitet werden können. Dies entspräche einer selbsterfüllenden Prophezeiung. Daher sind weitere, religiös neutrale“ Bonding- und Bridging-Aktivitäten einzubeziehen, mit denen eine differenzierte Betrachtung der jeweiligen Kombinationen unter Ausschluss religionsbezogener Variablen erfolgen kann.

zu treffen. Es ist jedoch von heterogenen Gruppen der Geflüchteten auszugehen (Herkunftsländer; kulturelle Werte), die den in den MSO involvierten Personen nicht bekannt sind und für die spontan vielfältige praktische, humanitäre und andere Hilfen geleistet werden (III. 7.1.4). Damit erscheinen die MSO im weitesten Sinne als Lernplattformen, die geflüchteten Menschen dabei helfen, soziale Kontakte zu knüpfen und Verständnis für die in Deutschland vorgefundenen Verhältnisse aufzubauen.

${ }^{79}$ Bereits die zu den Organisationszielen und Aktivitäten durchgeführten Explorativen Faktorenanalysen (EFA) (III. 7.1.3, Tab. 7.19; III. 7.1.4, Tab. 7.20) haben vor Augen geführt, dass die selektierten, analyserelevanten Variablen jeweils in unterschiedlichen Ausmaßen miteinander einhergehen können, ohne jedoch Auskunft über konkrete Kombinationen im Einzelfall geben zu können. In Hinblick auf konkrete Aussagen zur Bonding/Bridging-Performanz müssen sie daher noch einmal gesondert unter die Lupe genommen werden. 
Um die konkreten Kombinationen jeweils nachvollziehen und beschreibbar machen zu können, wurden für die Dimensionen Aktivitäten, Ziele und Selbstverständnisse jeweils nominalskalierte (,Sammel“-)Variablen erstellt. Die Validität der Datenzusammenstellung wurde mit Kreuztabellen überprüft.

Innerhalb der Bonding/Bridging-Auswertung besteht ein weiterer wichtiger Untersuchungsschritt darin, die separat abgefragten Mitgliedschaftsvoraussetzungen, Zielgruppen der Aktivitäten sowie Kooperationen zu berücksichtigen und im Rahmen der Analysedimension „Aufgeschlossenheit gegenüber der Umwelt“ in ihren Kombinationen und Häufigkeiten zu beschreiben (7.2.3). Dies erfolgt ebenfalls durch die Zusammenführung in einer nominalskalierten Variablen. Für diesen Analyseschritt sind die für die Mitgliedschaftsvoraussetzungen sowie Zielgruppen der Aktivitäten möglichen Antworten zunächst hinsichtlich ihrer Bonding-Kategorisierung unter die Lupe $\mathrm{zu}$ nehmen (7.2.3.1). Im Anschluss wird die durch die Häufigkeitsauszählung der Variablen abgebildete mehr oder weniger vorhandene „Aufgeschlossenheit gegenüber der Umwelt“ inhaltlich erläutert und interpretiert (7.2.3.2). Darauffolgend wird diese Dimension der Umweltoffenheit wiederum mit dem Aktivitätsspektrum der MSO sowie den spezifischen Typen von Kooperationspartnern, mit denen zusammengearbeitet wird, kontrastiert und auf Basis dieser Gegenüberstellung in Bezug auf Bridging-oder Bonding-Performanzen eine Typenbildung vorgenommen (7.2.4). ${ }^{80}$

Wie bereits erwähnt, wird im Verlauf der Auswertung in zwei Schritten ebenfalls kontrolliert, inwieweit in Hinblick auf die Angaben der MSO (In-)Kommensurabilitäten bzw. Widersprüche festzustellen sind. Dies betrifft insbesondere die Frage, inwieweit sich eine positive Selbsteinschätzung (Ziel der Allgemeinwohlorientierung; Selbstverständnis als Brückenbauer und Kulturdolmetscher) in der Praxis durch die Übernahme entsprechender Tätigkeiten erkennbar realisiert oder nicht.

${ }^{80}$ Anmerkung zum Auswertungsverfahren: Wie bereits thematisiert wurden für die Bearbeitung der Forschungsfrage in diesem Kapitel (7.2) unterschiedliche Analyseverfahren eingesetzt bzw. diese kombiniert. Als Ergänzung zur quantitativen Datenerhebung und auf Basis theoretischer Vorentscheidungen wurde ebenfalls ein qualitativ ausgerichtetes Auswertungsverfahren mit interpretativem Anteil gewählt. Mit dieser wurden die Kombinationen von analyserelevanten Variablen in ihrer Bedeutung für Bonding- und Bridging-Profile überprüft und auf dieser Basis eine Typenbildung der Einzelfälle vorgenommen. Damit sollte der Erkenntnisgewinn erhöht und ein differenziertes Bild vorzufindender Gegebenheiten gewonnen werden. Es handelt sich bei diesem Verfahren allerdings nicht um eines, das den elaborierten Analysemethoden der qualitativen Forschung zugeordnet werden kann, sondern um einen forschungspragmatischen Ansatz, der mit dem Anspruch verknüpft ist, die Erkenntnismöglichkeiten statistischer Analysen durch eine komplementäre Auswertungsmethode zu erweitern. 


\subsubsection{Das Zusammenspiel von bonding und bridging}

Im Folgenden werden Kombinationen und Verteilungen von Bonding- und Bridging-Ausrichtungen in Bezug auf ausgewählte Aktivitäten, Ziele und Selbstverständnisse der Organisationen dargelegt. Es handelt sich dabei jeweils um einander gegenübergestellte - gespiegelte - Variablen, die Bonding- und BridgingPerformanzen exemplarisch für alle MSO im Gesamtbild sowie säkulare im Vergleich zu religiösen in den Blick nehmen, und zwar in den Ausprägungsformen: nur Bonding (1) - nur Bridging (2) - Bonding und Bridging (3) - Weder-noch (4) (Abb. 7.18).

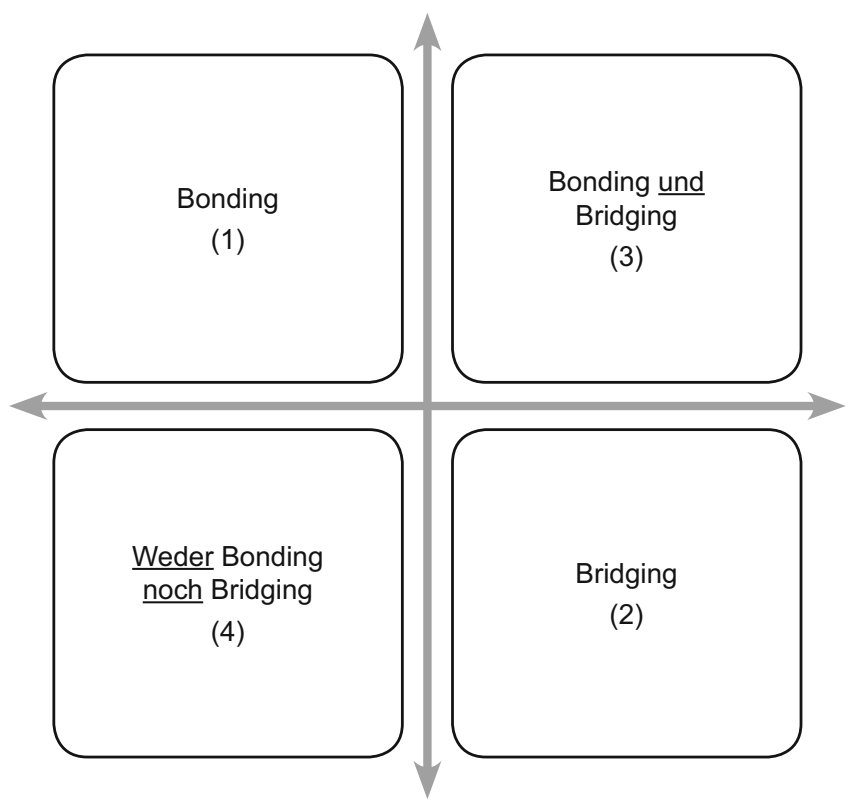

Abb. 7.18 Vier Ausprägungsformen von bonding und bridging in einer Vierfeldermatrix (Matrix-Felder 1 bis 4)

Die Abb. 7.18 soll verdeutlichen, dass bonding und bridging zumeist als widerstreitende, entgegengesetzte Phänomene konzipiert und als Antagonismen gedacht werden (Matrix-Felder 1,2), die einander aber nicht ausschließen. Bindungen nach innen und Brückenfunktionen nach außen können in der Praxis miteinander 
kompatibel sein, wobei das Zusammenwirken von bridging und bonding theoretisch denkbar ist (Matrix-Feld 3). Möglich ist allerdings auch die Negation eines solchen Modells: Weder bridging noch bonding ist vorhanden, entsprechend kann auch keine Verbindung eingegangen werden (Matrix-Feld 4). Insofern sind bridging und bonding hypothetische Instrumente, die das je Besondere eines sozialen Gebildes aber nicht vollständig und hinreichend erfassen können. Gleichwohl können Unterschiede in ihren Tendenzen ausgemacht werden.

In den folgenden Abschnitten und Unterkapiteln folgen nun detaillierte statistische Auswertungen, Beschreibungen, grafische Darstellungen und Interpretationen von Bonding- und Bridging-Erscheinungen. Tendenzen und Auffälligkeiten werden herausgestellt und insbesondere angesichts (sehr) geringer Fallzahlen tentative Schlussfolgerungen gezogen hinsichtlich der variierenden Bedeutung einzelner Subdimensionen für religiöse MSO im Kontext einer angenommenen kollektiven Identität bzw. eines kollektiven Schicksals.

In diesem Zusammenhang sei an dieser Stelle nochmals darauf hingewiesen, dass in der Analysestichprobe insgesamt 80 religiöse MSO vertreten sind, und zwar 44 islamische ${ }^{81}, 12$ alevitische, 17 christliche, 3 jüdische, 3 ezidische sowie eine hinduistische. Die jeweiligen Verteilungen der Variablen-Kombinationen auf die sechs Konfessionen werden ebenfalls beschrieben und interpretiert. Auf die dazugehörigen Tabellen, die im elektronischen Zusatzmaterial zu finden sind (Tab. A 5 bis A 11, Tab. A 13 bis A 17), wird jeweils in Fußnoten verwiesen.

Die folgenden statistischen Erfassungen und Zusammenstellungen resultieren in der Summe in den eingangs aufgeführten $>$ zentralen Ergebnissen. Auf diese wie auch die $>$ Zwischenfazite und die finalen Schlussfolgerungen (7.2.6 Fazit) sei der an komprimierten Befunden interessierte Leser verwiesen.

\section{- Spiegelung von Aktivitäten}

„Heimatpflege“ (Herkunftsländer) und „Stadtteilarbeit“

Die Abb. 7.19 zeigt für die Aktivitäten der Pflege der Herkunftskultur sowie der Stadtteilarbeit, dass die Heimatpflege allein (Matrix-Feld 1) von circa 20 Prozent der MSO getätigt wird. Die anderen drei Kombinationsformen (Felder 2-4) sind jeweils bei rund einem Viertel der MSO auf einem gleichen Niveau vorzufinden. ${ }^{82}$

\footnotetext{
${ }^{81}$ Von den insgesamt 44 im Sample vertretenen islamischen MSO weisen 28 (64\%) eine Verbandszugehörigkeit auf: 10 DITTIB, 7 IGMG, 5 IGBD, 2 VIKZ, 2 Islamische Religionsgemeinschaft NRW e. V. (IRG), 2 Verband engagierte Zivilgesellschaft in NRW e. V. (VEZ NRW) (darunter auch eine IGMG-MSO), 1 African Ummah Europe (7.1.1).

${ }^{82}$ Zum Umgang mit fehlenden Werten: Bei der Generierung der Sammel-Variablen bleiben fehlende Werte insofern unberücksichtigt, als sie als Nichtzustimmung (Residualkategorie)
} 
Beim Vergleich von religiösen und säkularen Vereinigungen zeigen sich bei der Bonding-Subdimension Heimatpflege keine nennenswerten Unterschiede. Bei der Bridging-Aktivität Stadtteilarbeit sind die religiösen weniger vertreten, dafür aber auf einem fast identischen Prozentniveau häufiger bei der Ausführung beider sowie keiner der gegenübergestellten Tätigkeiten (Abb. 7.19).

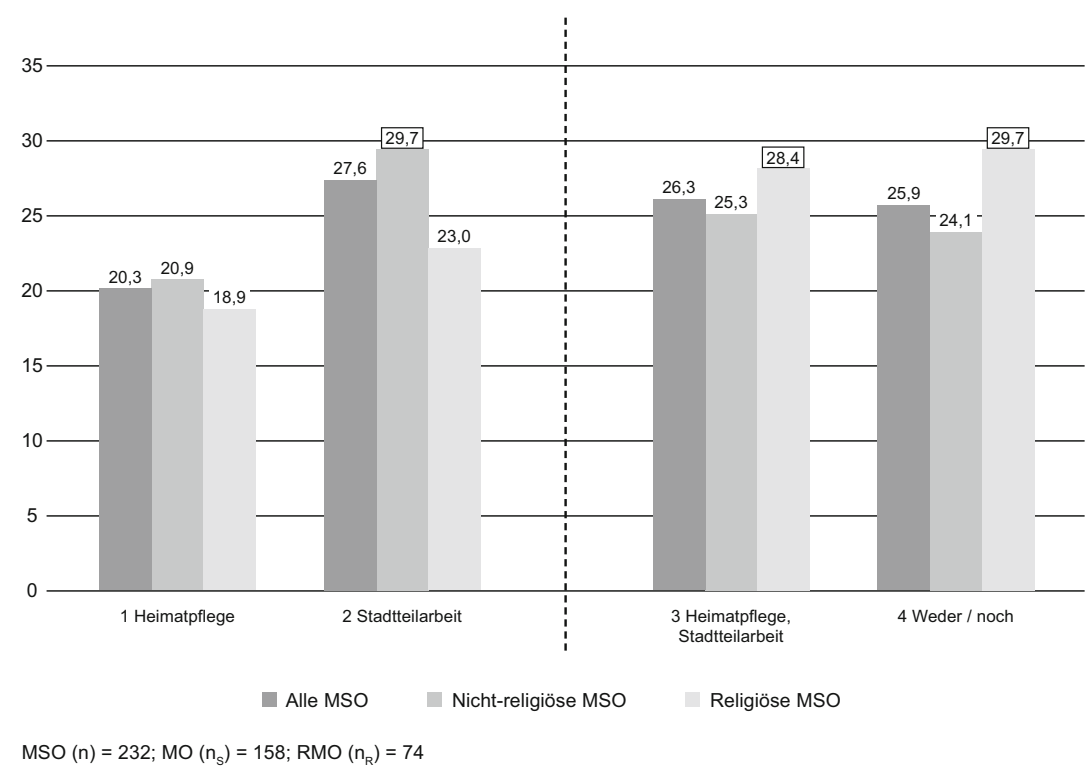

Abb.7.19 Verteilung der Aktivitäten „Heimatpflege“ \& „Stadtteilarbeit“ (in \%)

Hinsichtlich der Verteilung der unterschiedlichen Konfessionen auf die zwei einzelnen Aktivitäten einerseits (Matrix-Felder 1, 2) sowie ihrer Kombination und (Nicht-)Ausübung anderseits (Felder 3, 4) sind sehr ähnliche Zuordnungen ermittel$\mathrm{bar}^{83}$. Im Allgemeinen zeigt sich innerhalb der im Sample vorhandenen religiösen Traditionen ein disparater Stellenwert der Heimatpflege: Für jeweils ungefähr die Hälfte ist sie bedeutsam und für die andere nicht.

eingestuft werden, wenn sie auf einem der einbezogenen Subdimensionen auftreten. Weisen alle einkalkulierten Items einen fehlenden Wert auf, wird die Sammel-Variable insgesamt als fehlender Wert behandelt.

${ }^{83}$ Siehe zur Verteilung der beiden Aktivitäten nach religiösen Traditionen Tab. A 5 im elektronischen Zusatzmaterial. 
Für die islamischen, alevitischen und christlichen MSO lässt sich für alle vier Ausprägungsformen (Felder 1-4) eine breitere Streuung registrieren. Sie verweist auf verschiedenartige Ausrichtungen der religiösen Vereinigungen bei gleicher Konfession. Sie zeigt sich beim Islam auch hinsichtlich der (Nicht-)Ausübung von beiden Aktivitäten (Felder 3, 4) und einer bestehenden Verbandszugehörigkeit, die nicht als Indikator für das ein oder andere fungieren kann: So führen beispielsweise jeweils 4 DİTİB-Gemeinden beide Tätigkeiten (nicht) aus. Wenn zwei jüdische Gemeinden beide Tätigkeiten nicht ausüben, dieses aber zwei ezidische tun, kann nicht auf ein generelles (religiös verankertes) organisationales Handlungsmuster geschlossen werden.

Förderung der Herkunftssprache und der deutschen Sprache

Bei der Gegenüberstellung der Tätigkeiten zur Förderung der Herkunftssprache im Verhältnis zur Förderung der deutschen Sprache zeigen sich bei der Verrichtung von beiden Aktivitäten (Feld 3) durch jeweils circa 50 Prozent der drei separat betrachteten Organisationskategorien (alle, nicht-/religiöse) keine Unterschiede (Abb. 7.20). Dies gilt gleichermaßen für die Bonding-Aktivität der Herkunftssprachenförderung (Feld 1; je 8 bis $9 \%$ ). Das Lernen von Deutsch allein (Feld

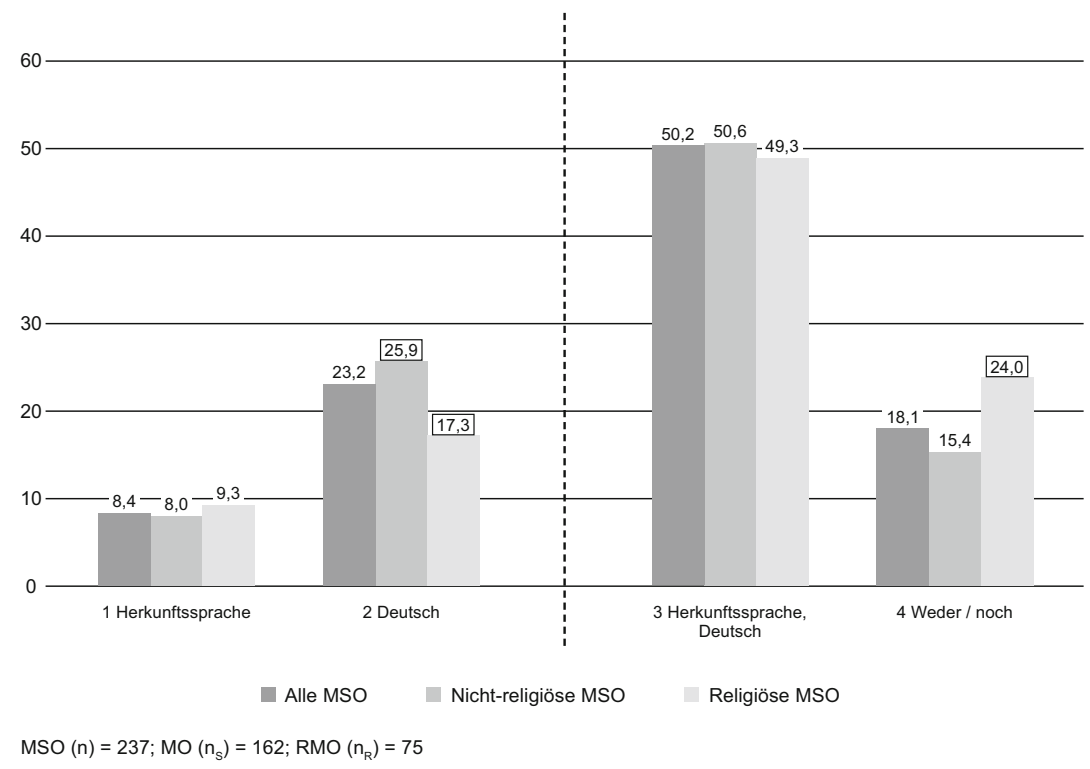

Abb. 7.20 Verteilung der Aktivitäten „Förderung Herkunftssprache“ \& „Förderung Deutsch“ (in \%) 
2) wird insgesamt häufiger angeboten als der Herkunftssprachenunterricht, seitens der religiösen MSO aber seltener (Feld 4; Differenz: 9 Prozentpunkte), und zwar von jeweils 6 islamischen und christlichen sowie einer alevitischen MSO. Im Programm der religiösen Vereinigungen ist auch häufiger keine der beiden Tätigkeiten vorzufinden (+9 Prozentpunkte): Auch hier betrifft dies insbesondere islamische und alevitische $\mathrm{MSO}^{84}$.

Beide Aktivitäten (Feld 3) werden zu unterschiedlichen Anteilen von allen im Sample vertretenen religiösen Traditionen ausgeführt: Darunter sind 50 Prozent der islamischen Vereinigungen, ein Drittel der alevitischen, ein gutes Viertel der christlichen sowie alle jüdischen und ezidischen und die hinduistische Gemeinde. Die Tatsache, dass die Hälfte der islamischen MSO das Lernen beider Sprachen anbietet und ein Viertel dies wiederum nicht tut, führt abermals ihre uneinheitlichen und variierenden Angebotsorientierungen vor Augen. Dies trifft auch für die alevitischen Vereine zu und für die christlichen sowohl hinsichtlich des Angebots der einzelnen als auch der zweifachen Sprachförderung.

Betrachtet man bei den islamischen MSO bezüglich der (Nicht-)Verrichtung beider Tätigkeiten zusätzlich wieder die Verbandzugehörigkeit, so lässt sich feststellen, dass 16 der insgesamt 22 Vereinigungen, die das Lernen beider Sprachen anbieten, einem Verband angehören. Insbesondere mit Blick auf die Zugehörigkeit zu DİTỉB zeigen sich hier aber wieder paritätische Verhältnisse in Bezug auf die (Nicht-)Förderung beider Sprachen (Nichtzustimmung / Zustimmung = 5:4).

„Religiöse Bildung/Erziehung“ und „Interreligiöser Dialog“

Die zwei religiös akzentuierten Aktivitäten „Interreligiöser Dialog“ und „Religiöse Bildung/Erziehung“ sind erwartungsgemäß v. a. für die religiösen MSO von Bedeutung (Abb. 7.21): Für rund 71 Prozent sind beide Tätigkeiten relevant (Feld 3), entsprechend selten - und zwar für nur eine christliche MSO - sind beide Aktivitäten gar nicht von Belang (Feld 4). Unter den Konfessionen, die religiöse Bildung anbieten und gleichzeitig dem interreligiösen Dialog nachgehen, befinden sich drei Viertel der islamischen (darunter 13 unabhängige und 20 verbandszugehörige Gemeinden), zwei Drittel der alevitischen, die Hälfte der christlichen, alle 3 jüdischen, 2 ezidische und die hinduistische MSO. Religiöse Bildung/Erziehung, für sich genommen (Feld 1), wird seitens religiöser Gemeinden deutlich häufiger vollzogen als der Dialog (Feld 2) ${ }^{85}$.

\footnotetext{
${ }^{84}$ Siehe zur Verteilung der beiden Aktivitäten nach religiösen Traditionen Tab. A 6 im elektronischen Zusatzmaterial.

${ }^{85}$ Siehe zur Verteilung der beiden Aktivitäten nach religiösen Traditionen Tab. A 7 im elektronischen Zusatzmaterial.
} 


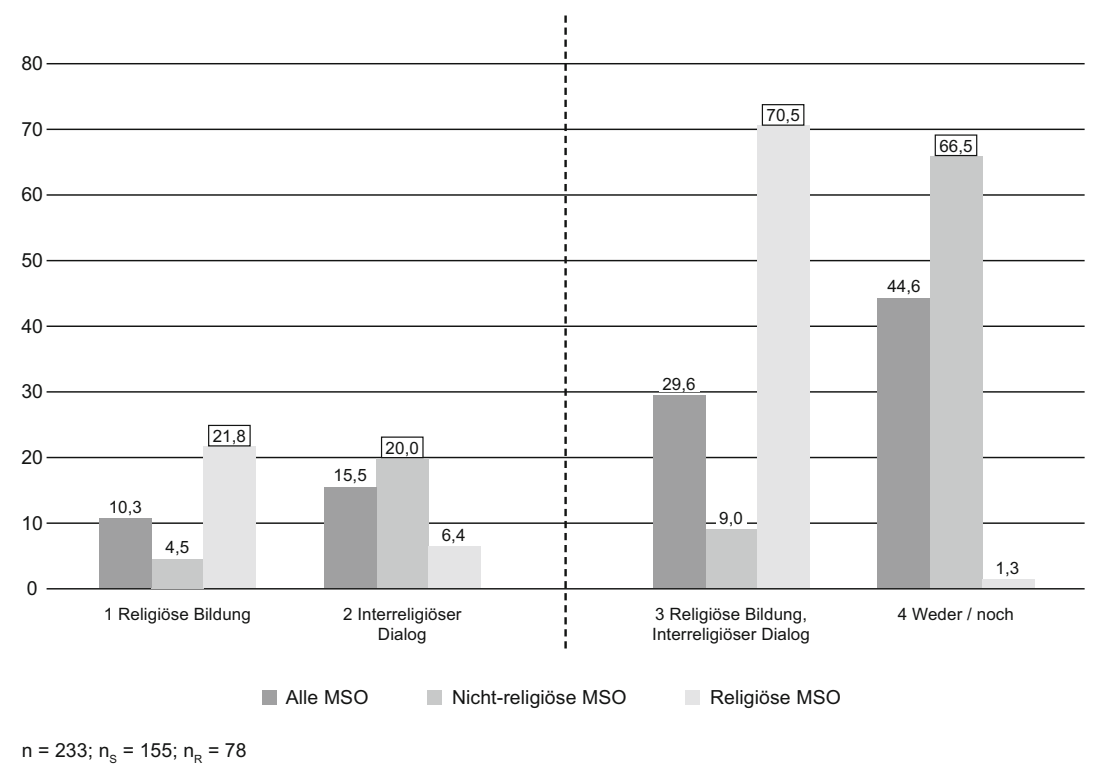

Abb. 7.21 Verteilung der Aktivitäten „Religiöse Bildung/Erziehung“ \& „Interreligiöser Dialog“ (in \%)

Hingegen spielen die beiden religiösen Aktivitäten für rund 67 Prozent der säkularen MSO gar keine Rolle. Wie bereits bei der Beschreibung der Tätigkeitsbereiche der MSO deutlich geworden ist (7.1.4), werden jedoch auch seitens säkularer Vereinigungen religiöse Angebote unterhalten: 31 der nicht-religiösen MSO (20\%) sind v. a. im interreligiösen Dialog aktiv, 14 übernehmen beide Tätigkeiten $(9 \%)$ und lediglich 7 bieten religiöse Bildung/Erziehung an $(4,5 \%)^{86}$.

\footnotetext{
${ }^{86}$ Hinweis zu den Prozentwerten: Insbesondere an dieser Stelle mag es auffallen, dass sich im Rahmen des hier gewählten Auswertungsverfahrens Prozentwerte ergeben, die von denjenigen in der deskriptiven Bestandsaufnahme (7.1) geringfügig abweichen. Dies ist auf die Behandlung von einzelnen fehlenden Werten als Nichtzustimmung zurückzuführen, denn dadurch ergeben sich divergierende Fallzahlen (n) für die nicht-religiösen $\left(n_{S}\right)$ und die religiösen $\left(n_{R}\right)$ MSO. Zur Erläuterung: Die deskriptive Auswertung (7.1.4) hat ergeben, dass 30 Prozent der nicht-religiösen MSO dem interreligiösen Dialog nachgehen $\left(n_{S}=150\right)$ und 14 Prozent der religiösen Bildung/Erziehung $\left(\mathrm{n}_{\mathrm{S}}=146\right)$. Im Rahmen der in diesem Kapitel gewählten Vorgehensweise $\left(\mathrm{n}_{\mathrm{S}}=155\right)$ wurden hinsichtlich der nicht-religiösen MSO neun fehlende Werte für die Aktivität der religiösen Bildung sowie fünf fehlende Werte für den interreligiösen Dialog unterschlagen, da sie als Nichtzustimmung gewertet werden.
} 
Die als säkular und damit als tendenziell religionsfern eingestuften MSO können sich de facto religiös gerieren, was sich insbesondere für die Bridging-Aktivität des interreligiösen Dialogs zeigt und auch bereits an anderer Stelle thematisiert wurde (7.1.4).

\section{- Illustration variierender Zusammenspiele von Bridging- und Bonding- Aktivitäten}

Im Folgenden soll das potenziell stark variierende Zusammenspiel kontrastierter Bonding- und Bridging-Aktivitäten aufgezeigt werden: Denn je nach Gegenüberstellung von jeweils spezifisch ausfallenden Tätigkeiten divergieren die vier Ausprägungsformen (Matrix-Felder 1 bis 4) für alle MSO im Gesamtbild sowie religiöse und säkulare im Speziellen.

„Heimatpflege“ (Herkunftsländer) und „Flüchtlingshilfe“

Wird die Bonding-Aktivität der Heimatpflege beispielsweise alternativ mit der Bridging-Tätigkeit der Flüchtlingshilfe gespiegelt, zeigen sich bei Betrachtung der religiösen und säkularen MSO deutlichere Differenzen: Religiöse MSO übernehmen beide Tätigkeiten einerseits zugleich häufiger (Feld $3 ;+5$ Prozentpunkte), anderseits zugleich auch in deutlich größerem Ausmaß gar nicht (Feld 4; +16 Prozentpunkte) (Abb. 7.22). Hier fällt auf, dass letzteres für alle jüdischen Gemeinden zutrifft. Für Aleviten spielt die Heimatpflege eine größere Rolle als die Flüchtlingshilfe; ein Viertel der alevitischen MSO verfolgt beide Tätigkeiten wiederum gar nicht ${ }^{87}$. Anhand der im Sample dominant vertretenen islamischen Organisationen zeigt sich hinsichtlich der beiden Aktivitäten abermals eine Streuung: Divergierende Verteilungen treten einerseits sowohl in Bezug auf die separate Ausführung der einzelnen Aktivitäten (Felder 1,2) als andererseits auch von beiden oder keiner der Tätigkeiten (Felder 3, 4) auf gleichgearteten Niveaus zutage.

Die säkularen Vereinigungen sind jeweils in der Heimatpflege (Feld 1) oder der Flüchtlingshilfe (Feld 2) häufiger engagiert (Abb. 7.22).

\footnotetext{
${ }^{87}$ Siehe zur Verteilung der beiden Aktivitäten nach religiösen Traditionen Tab. A 8 im elektronischen Zusatzmaterial.
} 


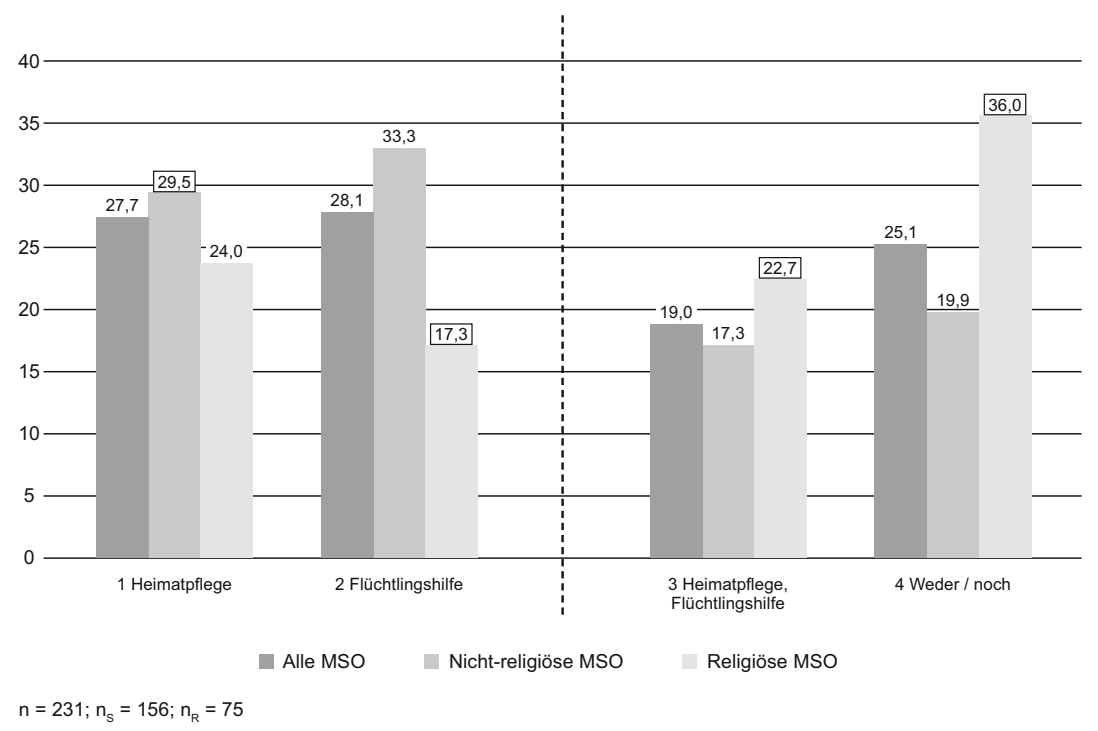

Abb. 7.22 Verteilung der Aktivitäten „Heimatpflege“ \& „Flüchtlingshilfe“ (in \%)

„Heimatpflege“ und „Internationale Arbeit“

Ein auf anderen Prozentniveaus angesiedeltes, aber ansonsten hinsichtlich der Verteilungen im Gesamtbild ähnliches Output (Felder 1-4) wie bei den zuvor gespiegelten Aktivitäten fördert die Gegenüberstellung der Heimatpflege mit der Internationalen Arbeit zutage, die z. B. Entwicklungszusammenarbeit, humanitäre Hilfe oder den Einsatz für Menschenrechte umfasst (Abb. 7.23).

Die religiösen MSO verfolgen mehrheitlich beide Aktivitäten zugleich (Feld 3), am häufigsten jedoch beide gar nicht (Feld 4). Allerdings zeigen sich zwischen den Konfessionen Unterschiede. ${ }^{88}$ Für ein Viertel der islamischen MSO spielen beide Tätigkeiten in Kombination eine Rolle, für die Hälfte jedoch gar nicht. Auf die jeweils hälftige (Nicht-)Relevanz der Heimatpflege für die religiösen Traditionen wurde bereits verwiesen. Für gut 40 Prozent der christlichen MSO, für über die Hälfte der alevitischen, für alle ezidischen Vereinigungen sowie die hinduistische Gemeinde ist Internationale Arbeit relevant.

${ }^{88}$ Siehe zur Verteilung der beiden Aktivitäten nach religiösen Traditionen Tab. A 9 im elektronischen Zusatzmaterial. 


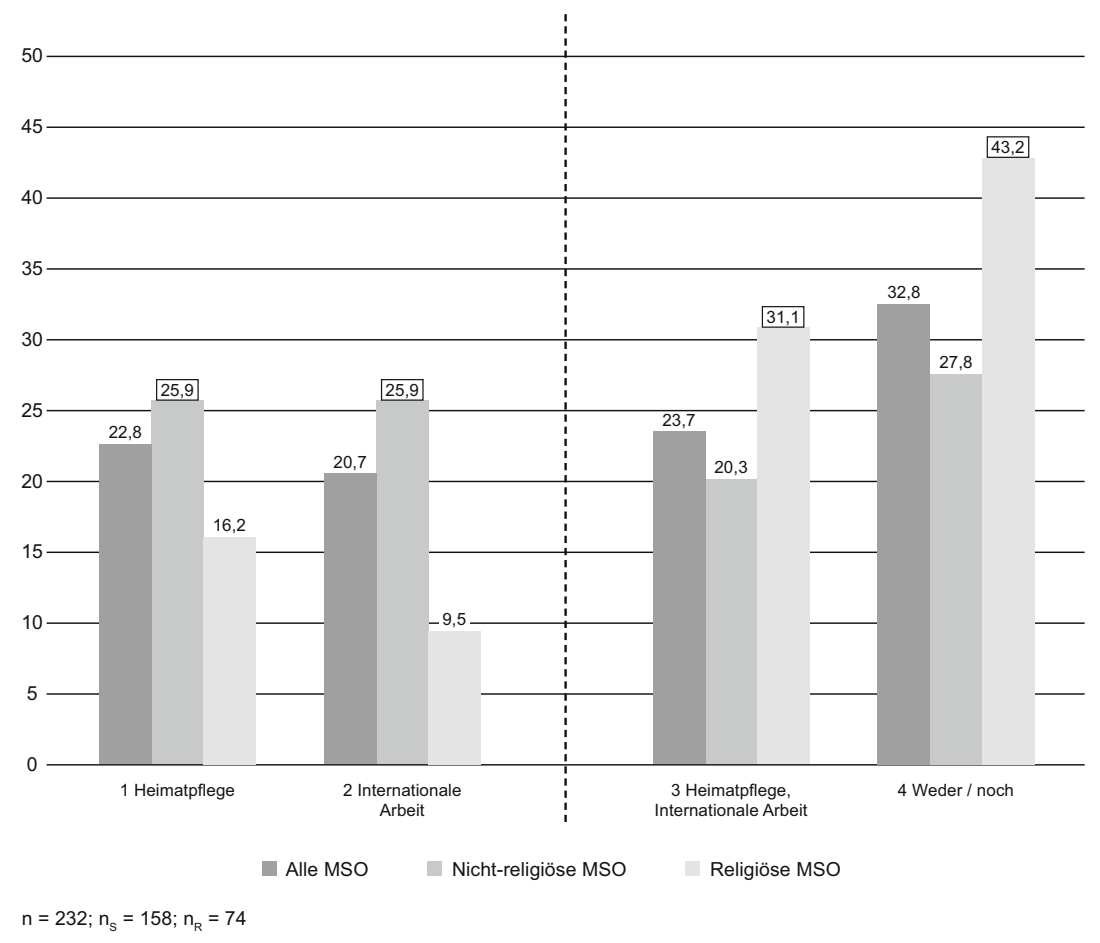

Abb. 7.23 Verteilung der Aktivitäten „Heimatpflege“ \& „Internationale Arbeit“ (in \%)

Aufgrund der spezifischen durch Verfolgung und Diskriminierung gezeichneten Schickale der (ethno-)religiösen Minderheiten der Aleviten und Eziden ist ihr Interesse an Internationaler Arbeit sehr nachvollziehbar. Insbesondere für die Aleviten und ihre zwei Dachverbände - die Alevitische Gemeinde Deutschland e. V. (AABF) sowie der Bund der Alevitischen Jugendlichen in Deutschland e. V. (BDAJ) - gehört der Einsatz für Menschenrechte, für die Rechte unterdrückter Minderheiten und für Antidiskriminierung, der insbesondere die Verbesserung der Situation der Aleviten in der Türkei betrifft, zu ihrem wesentlichen Ziel und Aufgabenfeld. ${ }^{89}$ Überraschenderweise spielt die Internationale Arbeit für alle drei jüdischen Gemeinden, deren Mitglieder aus Russland und der Ukraine kommen, keine Rolle. Dass sie bedeutsam

${ }^{89} \mathrm{https}$ ://alevi.com/unsere-ueberzeugungen/;www.bdaj.de/images/downloads/uu_pdf/sat zung/2015-Grundsatzprogramm_BDAJ.pdf (letzte Abrufe am 03.04.2021). 
ist, könnte angenommen werden angesichts der vielfältigen Migrationsgeschichten der in ihnen involvierten Personen, die seit Jahrtausenden Verfolgungen, Pogromen und dem nationalsozialistischen Völkermord, der Shoa, ausgeliefert waren. Andererseits kann ein solches im kollektiven Gedächtnis verankertes Schicksal natürlich auch in einem sich nach außen distanzierenden, zurückhaltenden Verhalten resultieren oder im Rahmen anderer Aktivitäten thematisiert und bearbeitet werden.

„Heimatpflege“ und „Arbeitsmarktzugang“

Wiederum anders verhält es sich hinsichtlich der Heimatpflege bei deren Spiegelung mit dem sehr spezifischen Bereich des Arbeitsmarktzugangs. In dem das strukturelle Integrationsbestreben betreffenden Feld sind die säkularen MSO deutlich aktiver als die religiösen Vereinigungen (Felder 2, 3), die innerhalb der Konstellation weniger brückenbauend bzw. in Bezug auf die Heimatpflege mehr unter sich agieren (Feld 1) (Abb. 7.24). Mit circa 42 Prozent üben die religiösen keine der beiden Aktivitäten aus (Feld 4). Insgesamt ist ein gutes Viertel der religiösen MSO - ohne und

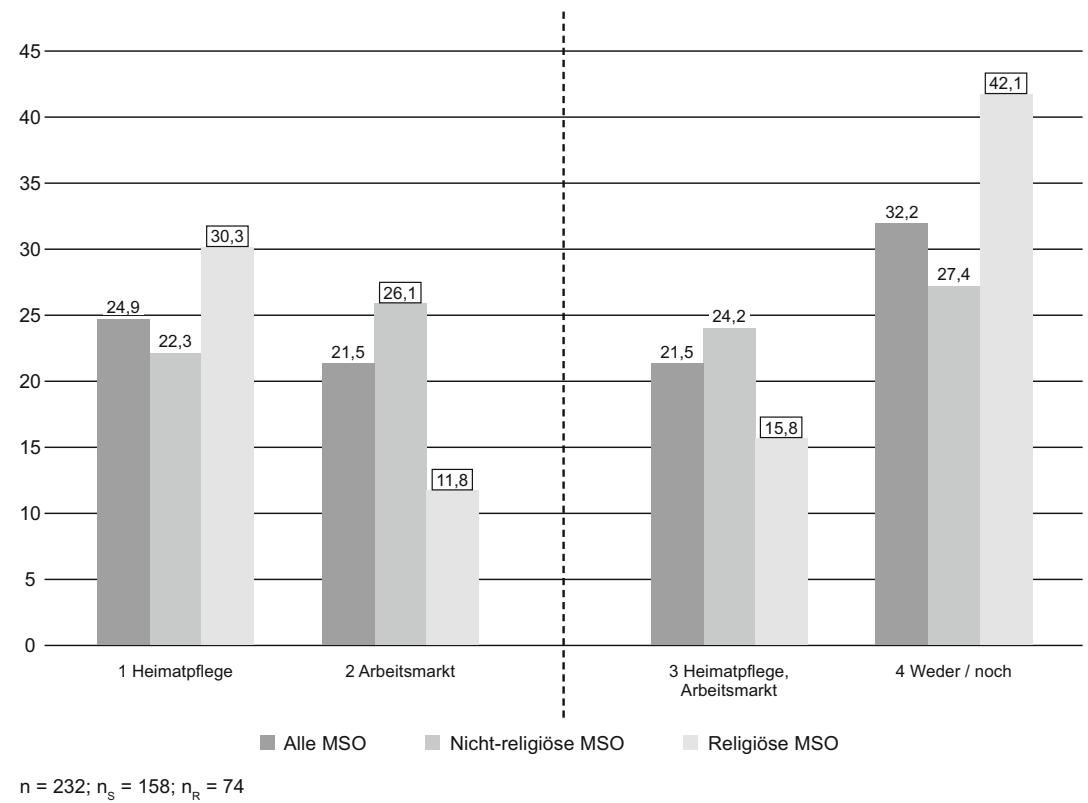

Abb. 7.24 Verteilung der Aktivitäten „Heimatpflege“ \& „Arbeitsmarktzugang“ (in \%) 
in Verbindung mit der Heimatpflege - im Feld der Arbeitsmarktintegration aktiv $(27,6 \%=11,8+15,8 \%)$. Unter diesen 21 religiösen MSO sind 13 islamische und damit ein knappes Drittel der muslimischen Gemeinden vertreten; des Weiteren 2 alevitische, 4 christliche und 2 ezidische Gemeinschaften ${ }^{90}$.

\section{- Zwischenfazit}

Bei Eliminierung religionsbezogener Variablen: Ausbalancierung von Bridgingund Bonding-Ausprägungen

Insgesamt zeigt sich bei den verschiedenen Kombinationen der Aktivitäten ein variierendes Zusammenspiel von bonding und bridging, die keine „EntwederOder“-Kategorien im Sinne bipolarer Gegensätze, sondern vielmehr „Mehr oder weniger"-Tendenzen darstellen (Putnam 2000: 23). Eine ausschließlich die religiösen Vereinigungen betreffende Bonding-Affinität lässt sich bei den hier berücksichtigten Aktivitäten nicht feststellen. Für ihre stärkeren Bonding-Ausprägungen ausschlaggebend sind allerdings ihr religiöser Charakter und die damit einhergehenden Bedarfe und Ausrichtungen. Insbesondere anhand der in der Analysestichprobe zahlenmäßig stärker vertretenen Konfessionen (Islam, Alevitentum, Christentum) wird sichtbar, dass sie sich nicht alle gleichmäßig auf die unterschiedlichen Variablen-Konfigurationen verteilen (was angesichts der disparaten Fallzahlen und grundsätzlich auch unwahrscheinlich wäre). Die uneinheitlichen und variierenden Aktivitätskombinationen lassen sich insbesondere in Bezug auf die islamischen MSO als regelmäßig in Erscheinung tretende Streuungen betrachten.

Für spezifische religiöse Traditionen in Bezug auf einzelne Kombinationen von Bridging- und Bonding-Items auszumachende Auffälligkeiten und ihre Interpretation (wie der jüdischen in Hinblick auf Internationale Arbeit) sind aufgrund der sehr geringen Fallzahlen mit Vorsicht und nicht als verallgemeinerbare organisationale Performanzmuster zu betrachten.

Eine Zusammenfassung der drei zu Beginn separat gespiegelten Aktivitätspaare ${ }^{91}$ nach den Zustimmungsraten entsprechend der Skalenpunkte „Trifft voll und ganz zu/Trifft eher zu“ fördert zutage, dass die religiösen MSO gleichzeitig mehr Bonding- und mehr Bridging-Ausprägungen aufweisen als die säkularen. Ebenfalls zeigt sich, dass die säkularen Vereinigungen in größerer Anzahl sowohl ausschließlich Bridging- als auch ausschließlich Bonding-Aktivitäten verfolgen (Abb. 7.25). Das Ergebnis überrascht keinesfalls, da in die Auszählung sowohl auf der Bridging-

\footnotetext{
${ }^{90}$ Siehe zur Verteilung der beiden Aktivitäten nach religiösen Traditionen Tab. A 10 im elektronischen Zusatzmaterial.

91 „Heimatpflege (Herkunftsländer)“ \& „Stadtteilarbeit“ I „Förderung der Herkunftssprache“ \& „Förderung der deutschen Sprache“ I ,Religiöse Bildung/Erziehung“ \& „Interreligiöser Dialog".
} 


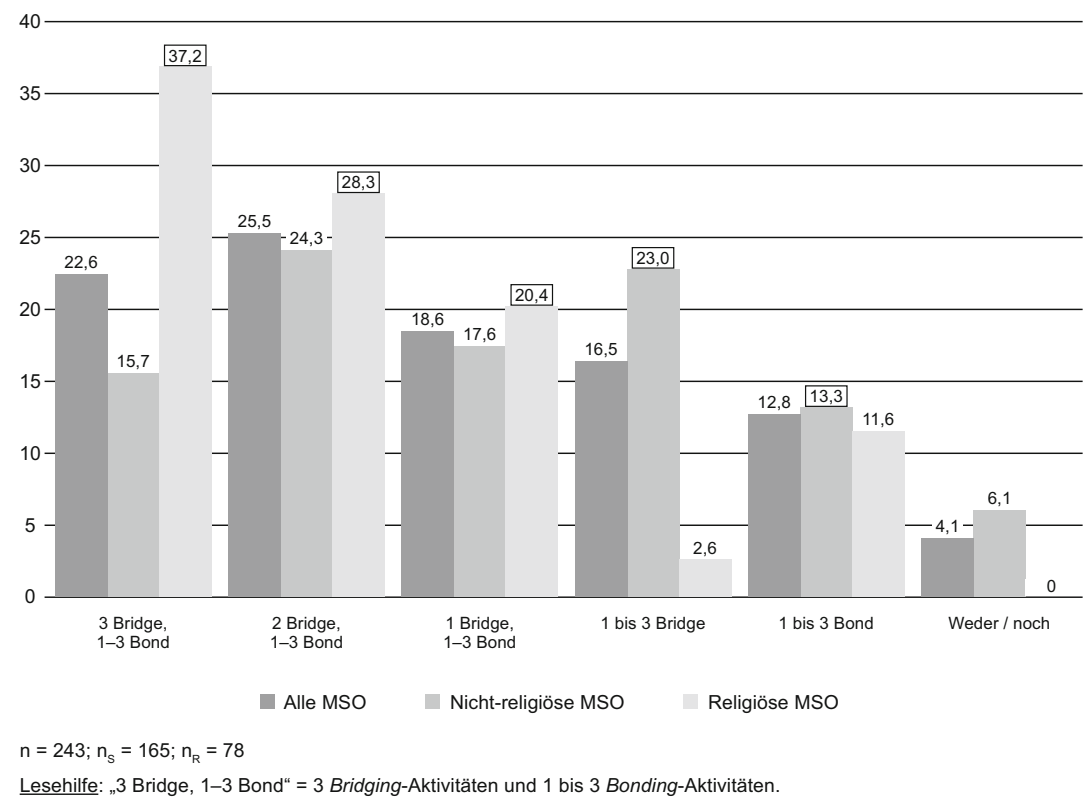

Abb. 7.25 Verteilung der drei Aktivitätspaare ${ }^{91}$ nach Bonding/Bridging-Ausprägungen (in $\%)$

als auch auf der Bonding-Seite jeweils ein religiös akzentuiertes Item eingeflossen ist. ${ }^{92}$ Insofern sind beide Sozialkapitalformen bei den religiösen Vereinigungen religiös konnotiert (,positive Teststrategie“").

Ein genauerer Blick auf die Ergebnisse des Zusammenspiels der Aktivitäten zeigt für die extremen Ausprägungen eines dreifachen Bonding lediglich eine Zustimmung für eine sich dem Alevitentum zuordnende religiöse MSO (siehe Tab. A 11 im elektronischen Zusatzmaterial). Die Kombination von ausschließlich drei Bridging-Tätigkeiten findet sich bei 5 säkularen, allerdings bei keiner der religiösen

92 „Religiöse Bildung/Erziehung“ \& „Interreligiöser Dialog“. 
Vereinigungen. 3 Bridging- und 3 Bonding-Aktivitäten lassen sich für 13 religiöse und 3 nicht-religiöse Organisationen feststellen. ${ }^{93}$

Berücksichtigt man bei der Aufsummierung zusätzlich die drei BridgingTätigkeiten der „Flüchtlingshilfe“, des ,Arbeitsmarktzugangs“ sowie der ,Internationalen Arbeit" zeigen sich ähnliche Ergebnisse: Mit Blick auf die hier einbezogenen Aktivitäten sind die religiösen MSO sowohl mehr bridging als auch mehr bonding zugleich (Tab. 7.30), was auch hier auf ihren religiösen Charakter bzw. die einbezogenen religiösen Aktivitäten zurückzuführen ist. Säkulare MSO verfolgen in dieser Konstellation deutlich häufiger ausschließlich Bridging-Tätigkeiten (Tab. 7.30).

Tab. 7.30 Kombination und Verteilung aller betrachteten Aktivitäten nach Bonding/Bridging-Ausprägungen (in \%)

\begin{tabular}{lccc}
\hline & Alle MSO & Nicht-religiöse MSO & Religiöse MSO \\
\hline 4-6 Bridge / 1 Bond & 8,8 & 9,4 & 7,5 \\
\hline 4-6 Bridge / 2 Bond & 14,0 & 14,1 & 13,8 \\
\hline 4-6 Bridge / 3 Bond & 8,0 & 2,4 & 20,0 \\
\hline$\sum$ 4-6 Bridge / 1-3 Bond & $\mathbf{3 0 , 8}$ & $\mathbf{2 5 , 9}$ & $\mathbf{4 1 , 3}$ \\
\hline 1-3 Bridge / 1 Bond & 15,2 & 13,5 & 18,8 \\
\hline 1-3 Bridge / 2 Bond & 18,4 & 19,4 & 16,3 \\
\hline 1-3 Bridge / 3 Bond & 4,0 & 1,2 & 10,0 \\
\hline$\sum$ 1-3 Bridge / 1-3 Bond & $\mathbf{3 7 , 6}$ & $\mathbf{3 4 , 1}$ & $\mathbf{4 5 , 1}$ \\
\hline 1-6 Bridge & 21,6 & 29,4 & 5,0 \\
\hline 1-3 Bond & 8,8 & 8,8 & 8,8 \\
\hline Weder / noch & 1,2 & 1,8 & 0 \\
\hline
\end{tabular}

$\mathrm{n}=250 ; \mathrm{n}_{\mathrm{S}}=170 ; \mathrm{n}_{\mathrm{R}}=80$.

Eliminiert man die religiösen Subdimensionen ${ }^{94}$ balancieren sich die Unterschiede aus und die säkularen und religiösen MSO weisen ähnliche Kombinationen von bridging und bonding auf. Es wird deutlich, dass der religiöse Charakter der Organisationen die Bridging-Performanz nicht relativiert. Säkulare MSO üben häufiger mehrere Bridging-Tätigkeiten aus, die nicht von Bonding-Aktivitäten flankiert sind. Bei den gestaffelten Kombinationen von einer oder zwei BondingAktivität(en) mit Bridging-Tätigkeiten zeigen sich bei beiden Organisationstypen ähnliche Verteilungen (Tab. 7.31).

93 Siehe für eine kleinteiligere Aufschlüsselung Tab. A 12 im elektronischen Zusatzmaterial. 94 „Interreligiöser Dialog“ und „Religiöse Bildung/Erziehung“. 
Tab. 7.31 Kombination und Verteilung der Aktivitäten (ohne religiöse Subdimensionen) nach Bonding/Bridging-Ausprägungen (in \%)

\begin{tabular}{lccc}
\hline & Alle MSO & Nicht-religiöse MSO & Religiöse MSO \\
\hline Weder / noch & 4,8 & 1,8 & 11,5 \\
\hline 1-2 Bond & 8,5 & 9,4 & 6,4 \\
\hline 1-2 Bridge & 12,5 & 11,8 & 14,1 \\
\hline 3-4 Bridge & 13,3 & 15,9 & 7,7 \\
\hline 5 Bridge & 2,8 & 4,1 & 0 \\
\hline$\sum$ 1-5 Bridge & $\mathbf{2 8 , 6}$ & $\mathbf{3 1 , 8}$ & $\mathbf{2 1 , 8}$ \\
\hline 1 Bond / 1-2 Bridge & 13,7 & 12,9 & 15,4 \\
\hline 1 Bond / 3-4 Bridge & 10,9 & 10 & 12,8 \\
\hline 1 Bond / 5 Bridge & 2,8 & 3,5 & $\mathbf{2 9 , 5}$ \\
\hline$\sum$ 1 Bond / 1-5 Bridge & $\mathbf{2 7 , 4}$ & $\mathbf{2 6 , 4}$ & $\mathbf{7 , 7}$ \\
\hline 2 Bond / 1-2 Bridge & 10,9 & 12,4 & $\mathbf{1 7 , 9}$ \\
\hline 2 Bond / 3-4 Bridge & 14,1 & 12,4 & 5,1 \\
\hline 2 Bond / Bridge & 5,6 & 5,9 & $\mathbf{3 0 , 7}$ \\
\hline$\sum$ 2 Bond / 1-5 Bridge & $\mathbf{3 0 , 6}$ & $\mathbf{3 0 , 7}$ & \\
\hline
\end{tabular}

$\mathrm{n}=248 ; \mathrm{n}_{\mathrm{S}}=170 ; \mathrm{n}_{\mathrm{R}}=78 \mid *=1$ Bond $=7,6 \%$ der MO, $2,6 \%$ der RMO 2 Bond $=1,8 \%$ der MO, $3,8 \%$ der RMO.

$* * *$

\section{- Spiegelung von Organisationszielen}

„Pflege Herkunftskultur“ und „Allgemeinwohlorientierung“

In der Gegenüberstellung dieser beiden Items kristallisiert sich die gesellschaftspolitisch virulente Debatte um Herkunfts- oder Aufnahmelandorientierungen migrantischer Vereinigungen.

Bei diesen beiden Zielen zeigt sich sehr deutlich die überwiegende gleichzeitige Befürwortung sowohl der Aufrechterhaltung bzw. Pflege der Herkunftssprache und -kultur als auch der Absichtserklärung, etwas für das Gemeinwohl der deutschen Gesellschaft zu tun (Feld 3; 58 bis $68 \%$ ) (Abb. 7.26). Die Kombination beider Ziele wird seitens der religiösen MSO in größerem Ausmaß bekräftigt (+10 Prozentpunkte), und zwar von über 60 Prozent der islamischen, fast allen alevitischen, von über der Hälfte der christlichen, allen ezidischen und jüdischen Gemeinden sowie der hinduistischen Vereinigung ${ }^{95}$. Für die säkularen MSO wird hingegen häufiger allein das Tätigsein für das Wohlergehen aller Gesellschaftsmitglieder bejaht

\footnotetext{
95 Siehe zur Verteilung der beiden Organisationsziele nach religiösen Traditionen Tab. A 13 im elektronischen Zusatzmaterial.
} 


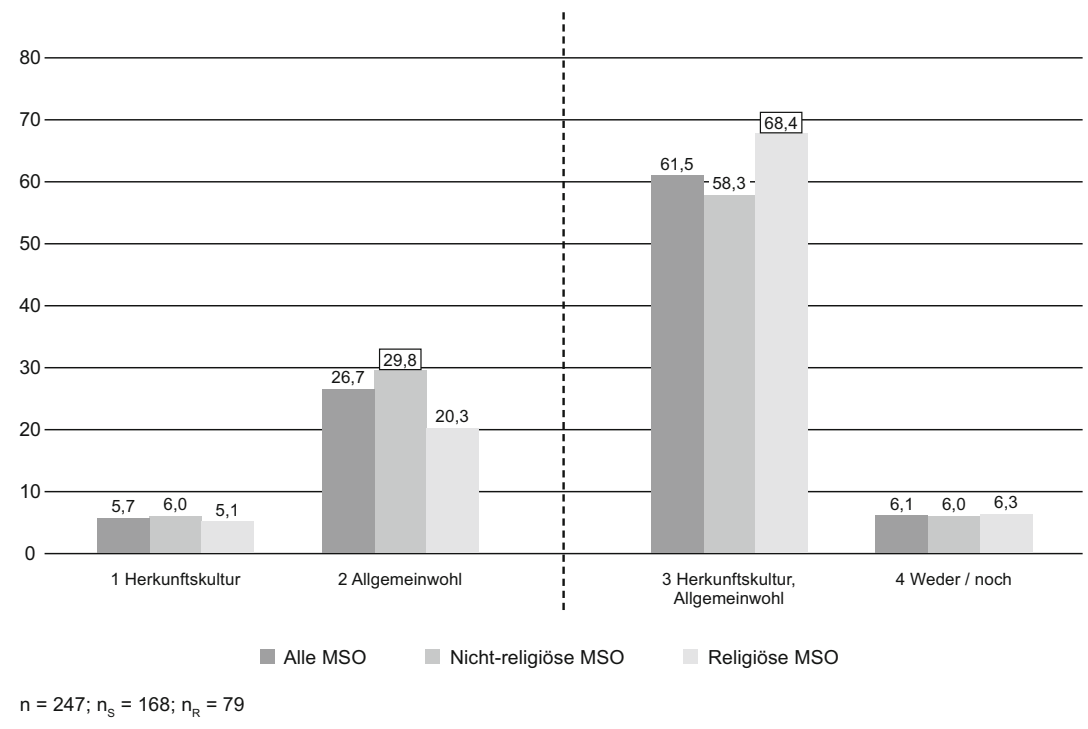

Abb. 7.26 Verteilung der Ziele „Pflege Herkunftskultur“ \& „Allgemeinwohlorientierung“ (in \%)

(30\% vs. $20 \%$ ). In Hinblick auf die Pflege der Herkunftskultur für sich genommen (Feld 1) sowie das Nicht-Zutreffen beider Organisationsziele (Feld 4) sind zwischen den einzelnen Organisationen gleich niedrige Prozentwertniveaus ohne nennenswerte Diskrepanzen festzustellen. Unter den religiösen MSO, die beide Ziele nicht befürworten, befinden sich 4 DİTİB-Gemeinden.

Dass die Pflege der Herkunftskultur für die religiösen MSO - in Kombination mit der auf Deutschland bezogenen Allgemeinwohlorientierung - relevanter ist, ist insofern nachvollziehbar, als Religion Bestandteil einer jeweiligen Kultur bzw. in diese eingebettet ist und durch sie in ihren sozialen Ausdrucksformen bestimmt wird. 
„Unterstützung politischer Positionen im Herkunftsland“ und „Unterstützung politischer Positionen in Deutschland“

Die beiden Organisationsziele parteipolitische Positionen in Deutschland mitzugestalten bzw. zu beeinflussen sowie in Deutschland im Herkunftsland vertretene politische Positionen zu unterstützen, werden von jeweils über drei Viertel aller MSO mehrheitlich verneint (Feld 4) (Abb. 7.27). Für das gleichzeitige Verfolgen beider Ziele lassen sich für die Vereinigungen Zustimmungsraten auf einem fast deckungsgleichen niedrigen Niveau ausmachen (Feld 3; 3 bis $4 \%$ ). Die deutsche Parteipolitik mitzugestalten ist für die säkularen MSO geringfügig relevanter als für die religiösen (Feld 2; +4 Prozentpunkte). Diesem Zweck wird allgemein häufiger zugestimmt als der Absicht, die Politik der Herkunftsländer zu unterstützen (Feld 1), was wiederum anteilig stärker seitens religiöser MSO bekräftigt wird: Konkret handelt es sich dabei um jeweils 3 alevitische und 3 christliche sowie lediglich eine islamische Vereinigung, die der Islamischen Gemeinschaft der Bosniaken

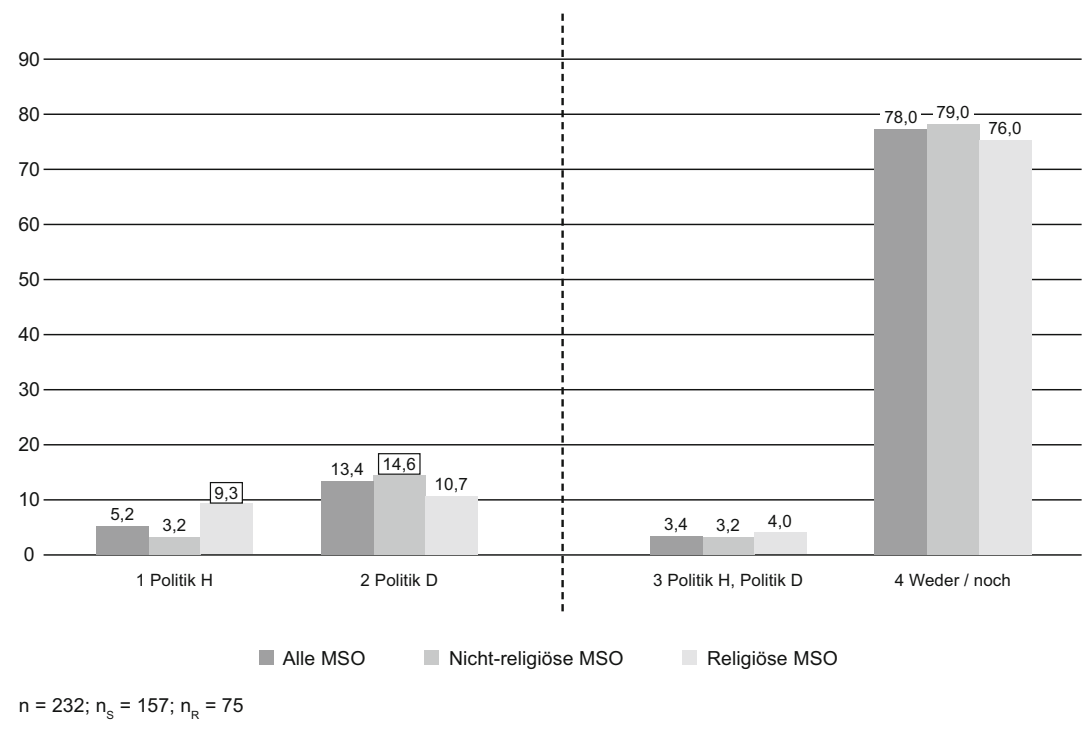

Abb.7.27 Verteilung der Ziele „Unterstützung pol. Posit. im Herkunftsland“ \& „Unterstützung pol. Posit. in Deutschland“ (in \%) 
in Deutschland e. V. (IGBD) angehört ${ }^{96}$. Es überrascht, dass diesem Ziel seitens der Vertreter türkisch-islamischer MSO nicht in größerer Anzahl zugestimmt wird. Diesbezüglich könnte angenommen werden, sich in Deutschland kontrovers geführten Debatten über politische Einflussnahmen der türkischen Politik entziehen und sozial erwünscht antworten zu wollen. Andererseits könnte die Politik im Herkunftsland für die befragten islamischen MSO tatsächlich nicht von Interesse sein oder eine Distanznahme vorherrschen.

Für die Aleviten und die Eziden zeigen sich innerhalb ihrer Gemeinden in etwa gleichen Anteilen exemplarisch sehr unterschiedlich ausgeprägte politische Interessen(-arrangements): Politisches Engagement steht apolitischem Verhalten gegenüber. Hinsichtlich der bei den alevitischen und ezidischen Vereinigungen festzustellenden Diskrepanzen können spezifische, zu unterschiedlichen Graden politisierte (Kollektiv-)Schicksale als Erklärungen herangezogen werden.

Die komplett unpolitische Ausrichtung der jüdischen Gemeinden könnte wiederum auf eine schwierige Vereinbarkeit von Jüdischsein und politischem Engagement hindeuten. Eine Nicht-Beteiligung an politischen (innerparteilichen) Debatten könnte mit Angst vor Antisemitismus, Anfeindungen, Israel-Kritik, aber auch spezifischen Familiengeschichten und Sozialisationserfahrungen zusammenhängen, die allesamt Konflikte verursachen können, bei denen ein wie auch immer geartetes politisches Engagement lieber vermieden wird.

Mischungsverhältnisse dreier Bonding-Zielorientierungen

Betrachtet man die drei Bonding-Ziele 1.) Verbesserung der Lebenssituation und Teilhabe von Zugewanderten (Landsleuten) in Deutschland, 2.) gemeinsame Religionspraxis und 3.) Vertretung bzw. Sichtbarmachung eigener Interessen, so zeigt sich, dass über 50 Prozent der MSO $(34,8 \%+20 \%)$ zwei oder drei der Ziele zustimmen. Deutlich erkennbar ist, dass sich die religiösen MSO in Hinblick auf die Bekräftigung von allen drei Zielen stärker in Richtung bonding bewegen (Abb. 7.28).

Konkret zeigt sich diese dreifache, religiös konnotierte Bonding-Performanz im Sample für fast die Hälfte der islamischen, über 80 Prozent der alevitischen, ein Drittel der christlichen, alle ezidischen und jüdischen Vereinigungen sowie die hinduistische Gemeinde ${ }^{97}$. Die Zusammenstellung der drei Ziele verdeutlicht, dass für die alevitischen MSO die Artikulation gruppenbezogener Interessen und Positionen im Sinne einer Mitbestimmung und öffentlichen Einflussnahme bedeutsam ist,

\footnotetext{
${ }^{96}$ Siehe zur Verteilung der beiden Organisationsziele nach religiösen Traditionen Tab. A 14 im elektronischen Zusatzmaterial.

${ }^{97}$ Siehe zur Verteilung der drei Bonding-Organisationsziele nach religiösen Traditionen Tab. A 15 im elektronischen Zusatzmaterial.
} 


$$
60
$$

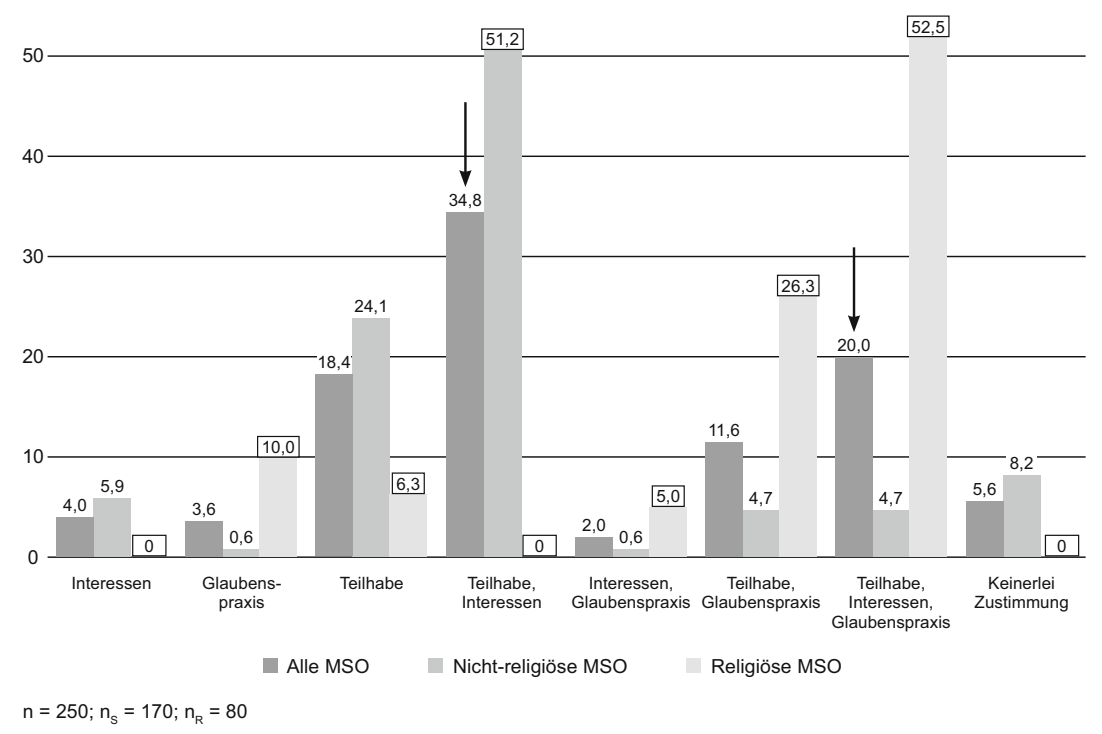

Abb. 7.28 Verteilung von drei Bonding-Zielen (in \%)

wohingegen bei den christlichen Vereinigungen tendenziell die Teilhabe im Sinne eines nachteilsausgleichenden gesellschaftlichen Einbezogenseins relevanter ist.

Der Zweck der Glaubenspraxis für sich genommen ist nur für 10 Prozent der religiösen Vereinigungen - 7 islamische und eine christliche - relevant und wird häufiger von den beiden anderen Zielen flankiert. 6 Prozent der religiösen MSO stimmen ausschließlich dem Ziel der Teilhabestärkung zu. Ansonsten sind das gesellschaftliche Beteiligtwerden und die Interessenvertretung sowohl für religiöse als auch säkulare MSO gleichbedeutend. ${ }^{98}$ Wird damit in einem weiteren Sinn das Anliegen einer chancengleichen - ökonomischen, kulturellen, sozialen, politischen - Teilhabe verstanden, so sind damit zentrale integrationspolitische Belange impliziert, wobei deren tatsächliche Realisierung Kommunikation und Austausch (Vernetzung) mit

\footnotetext{
${ }^{98}$ Wird von den drei betrachteten Bonding-Zielorientierungen das Ziel der Interessenvertretung ausgeschlossen, wird hinsichtlich des Zusammenspiels der beiden Zwecke „Gemeinsame Glaubenspraxis“ und „Teilhabeförderung von Zugewanderten (Landsleuten)“ die Relevanz der religiösen Traditionszuordnung und -pflege noch offensichtlicher: Die beiden Ziele sind für 79 Prozent der religiösen Vereinigungen bedeutsam. Auf einem ähnlichen Prozentniveau (77 \%) ist die nachteilsausgleichende Teilhabeförderung für säkulare MSO bedeutsam.
} 
anderen gesellschaftlichen Gruppen erforderlich macht und sich diese wiederum in den Aktivitäten und im Kooperationsverhalten der MSO spiegeln. Inwieweit zwischen letzteren und den mitgeteilten Organisationszielen Diskrepanzen (,Inkommensurabilitäten“) festzustellen sind, wird im Rahmen dieser Auswertung ebenfalls geprüft.

\section{- Spiegelung von Selbstverständnissen}

„Selbsthilfeorganisation“ und „Brückenbauer/Kulturdolmetscher“

Bei einer Gegenüberstellung der Bridging-Selbsteinschätzung, ein Brückenbauer/Kulturdolmetscher zu sein, sowie der Bonding-Selbstinterpretation, als Selbsthilfeorganisation zu fungieren, ist für 50 Prozent der MSO eine Kombination der beiden Selbstkonzepte zu konstatieren (Feld 3) (Abb. 7.29). Für jeweils rund 38

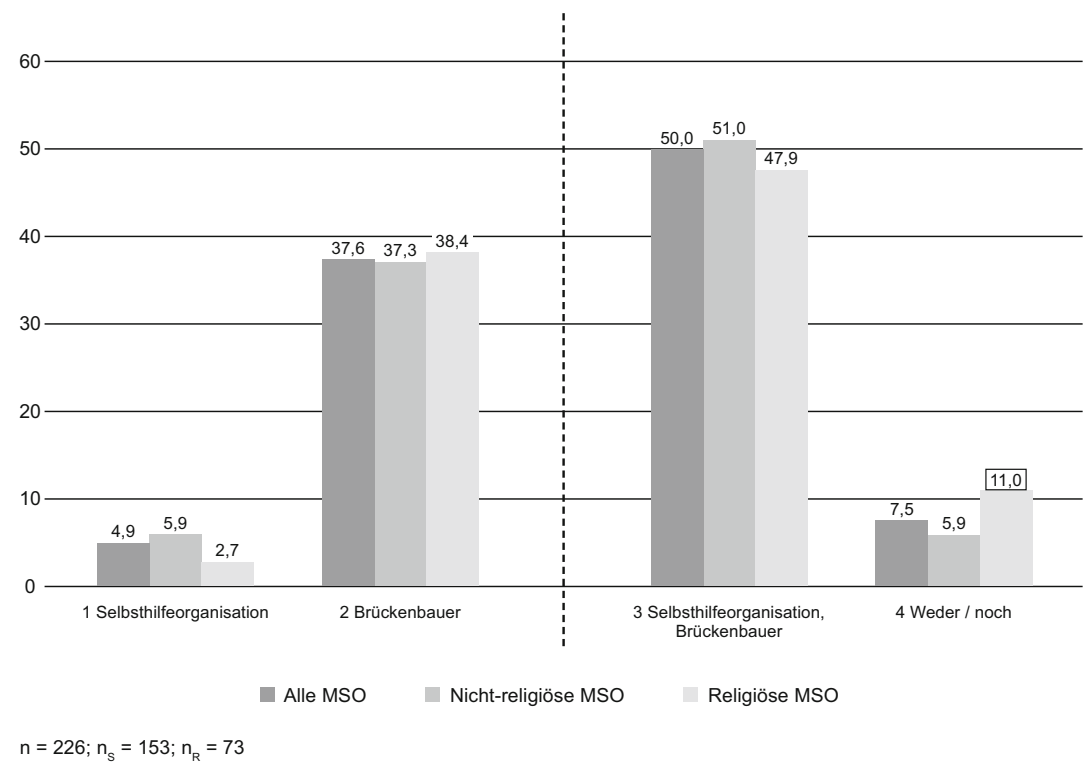

Abb. 7.29 Verteilung der Selbstverständnisse „Selbsthilfeorganisation“ \& „Brückenbauer“ (in \%) 
Prozent der separat inspizierten MSO-Typen zählt allein die Vorstellung, eine zwischen Kulturen brückenbauende Vereinigung zu sein (Feld 2). Die Spiegelung der beiden Variablen zeigt, dass sich religiöse MSO um rund 10 Prozentpunkte häufiger als beides denn ausschließlich als Brückenbauer verstehen. Hier zeigt sich hinsichtlich der verschiedenen Konfessionen eine recht gleichmäßige Verteilung auf beide Ausprägungsvarianten (Felder 2, 3), sodass gleiche religiöse Traditionen (vom Ezidentum abgesehen) bezüglich ihrer Selbstbilder differieren ${ }^{99}$. Im Vergleich zu den säkularen ordnen sich die religiösen MSO beiden Selbstverständnissen geringfügig seltener und etwas häufiger keinem der beiden zu (Feld 4) (Abb. 7.29). Tendenziell betrachten sie sich eher als Brückenbauer denn als Selbsthilfeorganisationen.

„Selbsthilfeorganisation“, „Brückenbauer“ und „Integrationspromotor“

Fügt man den beiden Selbstinterpretationen das ostentative Bridging-Selbstbild eines Integrationspromotors hinzu, dann zeigen sich insgesamt hinsichtlich der Zustimmung zu allen drei Zielen - wie auch bei der vorigen Kontrastierung der zwei Selbstverständnisse - für die jeweils unterschiedenen MSO-Typen fast übereinstimmende Prozentwerte (43-44 \%; ohne Abb.). Zudem ist ersichtlich, dass sich die religiösen MSO häufiger als Brückenbauer und Integrationspromotor zugleich begreifen (RMO: 32 \% vs. MO: $26 \%$ ). Entweder als Brückenbauer oder Integrationsförderer verstehen sich vor allem säkulare Vereinigungen, aber insgesamt auf einem niedrigen Prozentwertniveau (9,2\%; 6,1\%).

Hinsichtlich der (Nicht-)Zustimmung eine Selbsthilfeorganisation zu sein, unterscheiden sich Islam, Aleviten-, Christen- und Judentum kaum: Bei den vier Konfessionen sind mit Blick auf die Kombinationen „Brückenbauer und Integrationspromotor“ sowie „Brückenbauer, Integrationspromotor und Selbsthilfeorganisation" fast paritätische Verteilungen festzustellen ${ }^{100}$. Auch hier sind innerhalb der religiösen Traditionen also variierende Selbstzuschreibungen ersichtlich.

$$
* * *
$$

Überprüfung von (In-)Kommensurabilitäten I: Selbstdarstellung versus Aktivitäten

Wie eingangs bei der Erläuterung der Vorgehensweise angekündigt soll im Folgenden nun abgeglichen werden, inwieweit sich die positive Selbstdarstellung der MSO in der Übernahme spezifischer Bridging-Aktivitäten spiegelt. Insgesamt in den Blick genommen werden dafür die Zustimmungen zum Ziel „Etwas für das

\footnotetext{
${ }^{99}$ Siehe zur Verteilung der beiden Selbstverständnisse nach religiösen Traditionen Tab. A 16 im elektronischen Zusatzmaterial.

100 Siehe zur Verteilung der drei Selbstverständnisse nach religiösen Traditionen Tab. A 17 im elektronischen Zusatzmaterial.
} 
allgemeine Wohl in Deutschland zu tun" sowie zu den vier Selbstverständnissen „Brückenbauer und Kulturdolmetscher“, „Integrationspromotor“, „Dienstleister“ oder „Förderorganisation“ zu sein. Für 152 MSO $(60,3 \%=29 \%+31,3 \%)$ und damit mehrheitlich werden 3 oder 4 der das Ziel der Allgemeinwohlorientierung sowie integrationsfördernde Selbstdefinitionen betreffenden Items bejaht (Abb. 7.30).

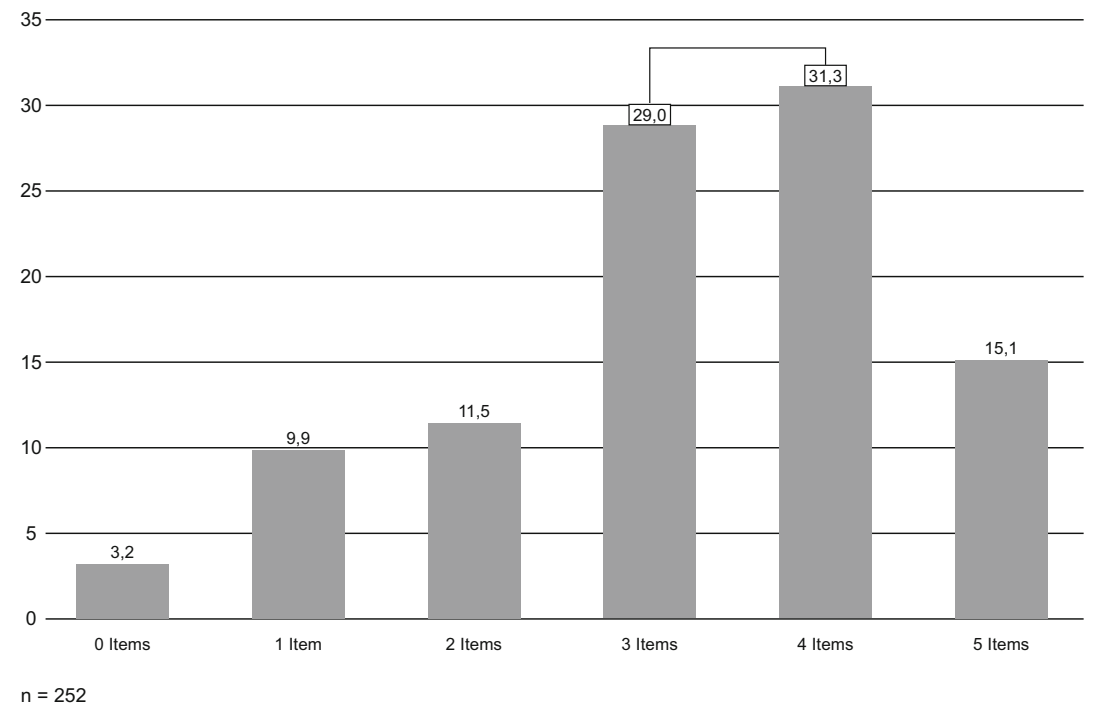

Abb.7.30 Zustimmungsraten zu maximal 5 Items positiver Selbstdarstellung (in \%)

Die Allgemeinwohlorientierung ist am ehesten anhand von Bridging-Aktivitäten abzulesen; in einem zweiten Schritt lassen sich anhand der Auskünfte der Funktionsträger Diskrepanzen zwischen organisationalen Selbstbildern, Aktivitäten, der Festlegung von Zielgruppen und Mitgliedschaftsvoraussetzungen sowie dem Kooperationsverhalten feststellen.

Das Ziel der Allgemeinwohlorientierung ist normativ besonders aufgeladen. Es stellt einen Aspekt der fünf Kriterien umfassenden Definition des Engagementbegriffs dar (II. 3.1). Grundsätzlich ist Allgemeinwohlorientierung schwer zu messen. ${ }^{101}$ Diesbezüglich wird im Freiwilligensurvey konstatiert, dass zur Beurteilung die Intention der Personen in den Blick zu nehmen sei (Simonson et al.

101 „Allgemeinwohl“ oder „Gemeinwohl“ wird allgemein verstanden als ein Gegensatz zu bloßen Privat- oder Partikularinteressen von einzelnen Personen oder Gruppen innerhalb 
2016a: 28), dass die Gemeinwohlorientierung von Aktivitäten letztlich vorausgesetzt werden müsse (ebd.: 31) oder anhand ,der Zielsetzung der Organisation oder Einrichtung, in der [die] freiwillige Tätigkeit ausgeübt wird, erkennbar" sei (Simonson et al. 2016b: 72). In Bezug auf die in dieser Arbeit untersuchte Mesoebene bietet dieses Statement hinsichtlich der Prüfung von Allgemeinwohlorientierung keine Lösung an. Für die vorliegende Analyse soll ausschlaggebend sein, dass die Tätigkeiten die Grenzen der eigenen Gemeinschaft überschreiten, anderen Gesellschaftsmitgliedern von Nutzen sind und durch die Generierung von positiven externen Effekten die gesellschaftliche Kohäsion stärken (BMFSFJ 2012: 33).

Der vorgenommene Abgleich geht über eine Gegenüberstellung der Selbstpräsentation mit den sechs als Bridging-Aktivitäten kategorisierten Variablen ${ }^{102}$ hinaus und bezieht auch die fünf potenziell brückenschlagenden sozialarbeiterischen und erzieherischen Tätigkeiten ${ }^{103}$ mit ein. Denn eine MSO, die z. B. ausschließlich Kinder- und Jugendarbeit durchführt, kann sich als Brückenbauer oder Integrationspromotor verstehen bzw. diese Rollen tatsächlich übernehmen.

Entsprechend der im Rahmen der Auswertung bereits dargelegten sehr hohen Aktivitätsraten der MSO (7.1.4) zeigt die Nebeneinanderstellung der beiden Tätigkeitskategorien, dass 83 Prozent der Vereinigungen in unterschiedlichen Ausmaßen und Kombinationen sowohl andere Gruppen adressierende (bridging) als auch (sozial-)pädagogische Aktivitäten übernehmen (Abb. 7.31).

Im Gesamtbild zeigt der Abgleich der positiven Selbstbilder mit den Aktivitäten, dass für 233 MSO (91\%; $\left.\mathrm{n}=256^{104}\right)$ Übereinstimmungen festzustellen sind. 16 weitere MSO (6\%) stellen sich positiv dar und übernehmen sozialarbeiterische oder allgemeine Bildungstätigkeiten. Für lediglich 7 Vereinigungen (3\%) lassen sich vorerst Diskrepanzen ausmachen. Die in den 7 Fällen zu vernehmenden Widersprüchlichkeiten betreffen ein (sehr) positives Selbstverständnis, dessen praktische Realisierung sich jedoch überhaupt nicht in der Übernahme der hier fokussierten Bridging- oder sozialpädagogischen Tätigkeiten spiegelt. Darunter ist eine religiöse MSO, die dem Alevitentum zugeordnet wird. Berücksichtigt man bei diesen Fällen zusätzlich die weder bonding noch bridging oder der Sozialpädagogik zugeordneten Tätigkeiten - wie etwa Kultur, Sport, Freizeit und Politik - relativiert sich die

einer Gesellschaft. Letztendlich geht es darum, sich für öffentliche Belange und gesellschaftlichen Umgang einzusetzen und dieses Bemühen einer Beschränkung auf das Private und individuellen Nutzen vorzuziehen.

102 Stadtteilarbeit, Interreligiöser Dialog, Deutschförderung, Flüchtlingshilfe, Internationale Arbeit, Arbeitsmarktzugang.

103 Elterngruppen/Familienarbeit; Kinder- und Jugendarbeit; Seniorenarbeit; Mädchen- und Frauenarbeit; Bildung/Erziehung allgemein.

${ }^{104}$ Eine Organisation fällt aus dem Sample heraus, da sie sich um den Tierschutz kümmert und sich als keine Menschen adressierende Hilfsorganisation versteht. 


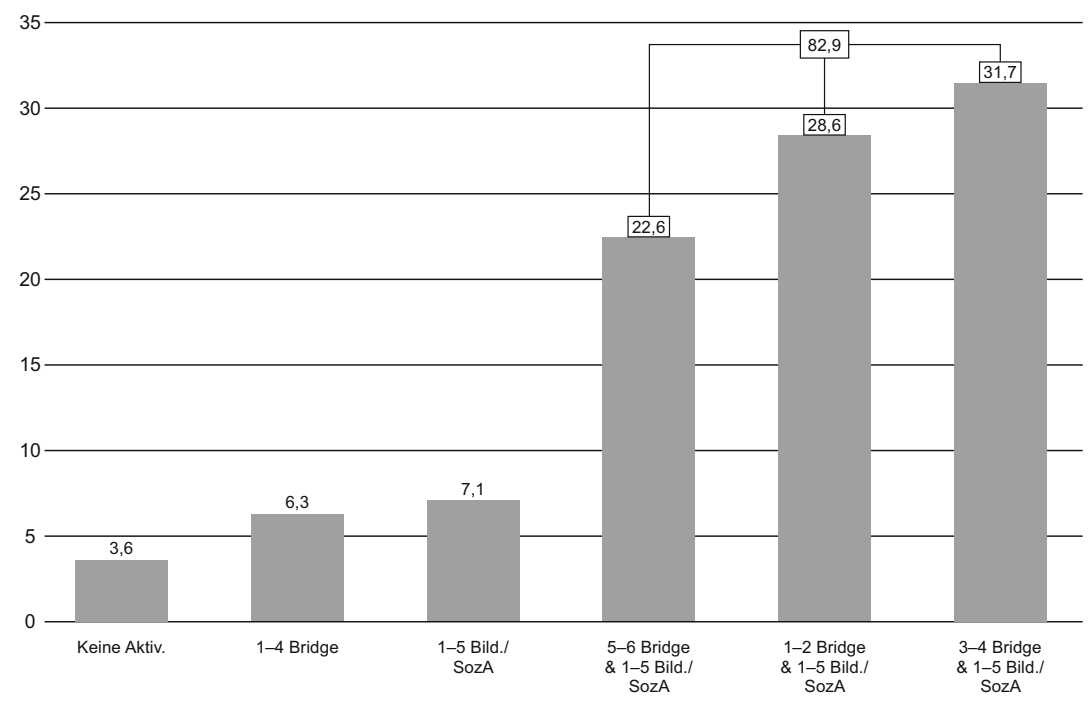

$\mathrm{n}=252$

Abkürzungen: „Bild./SozA“ = Bildung allgemein und/oder erzieherische/sozialarbeiterische Aktivitäten; "Bridge" = Bridging-Aktivitäten.

Lesehilfe: „Keine Aktiv.“ = keine Bridging-, Bildungs- oder erzieherischen Aktivitäten; „1-4 Bridge“ = 1 bis 4 BridgingAktivitäten; „1-5 Bild./SozA“ = 1 bis 5 Aktivitäten im Bereich Bildung allgemein bzw. erzieherische/sozialarbeiterische Aktivitäten.

Abb. 7.31 Verhältnisbestimmung zwischen dezidiert brückenbauenden und (sozial-) pädagogischen Aktivitäten der MSO (in \%)

Registrierung mangelnder Übereinstimmungen insofern, als alle 7 MSO mehrheitlich in zwei der Bereiche aktiv sind. Ein weiterer prüfender Blick zeigt dann, dass in vier der betreffenden Fälle durchaus von einer Kongruenz zu sprechen ist: Warum sollten sich MSO, die lediglich Kultur-, Freizeit- oder Sportangebote unterbreiten, nicht als Brückenauer verstehen oder das Ziel angeben, etwas für das allgemeine Wohl in Deutschland tun zu wollen? Da über die Offenheit der MSO an dieser Stelle noch keine Aussage getroffen werden kann, werden die benannten 7 Fälle in einem zweiten Schritt noch einmal gesondert im Zusammenhang mit der Dimension ,Aufgeschlossenheit der MSO gegenüber ihrer Umwelt" untersucht; diese umfasst die Mitgliedschaftsvoraussetzungen, Zielgruppen sowie das Kooperationsverhalten.

An dieser Stelle sind unter den kongruenten Fällen interessante Antwortverhalten hervorzuheben. In 10 Fällen $(4 \%)$ - darunter 5 religiöse ${ }^{105}-$ können den MSO

${ }^{105}$ Zwei islamische, eine alevitische, eine griechisch-orthodoxe und eine mennonitische. 
eine gewisse Bescheidenheit und ,Understatements“ attribuiert werden, da zahlreiche Bridging- oder sozialarbeiterische Tätigkeiten übernommen werden, aber dem Ziel der Gemeinwohlorientierung sowie den positiven Selbstverständnissen überhaupt nicht oder in Anbetracht des Umfangs der Tätigkeiten nur in sehr modester Art zugestimmt wird. In 5 weiteren Fällen wird ein scheinbar realitätsgerechtes, unprätentiöses Antwortverhalten deutlich. In den 5 betreffenden MSO gehen Aktivitäten der religiösen Bildung und Brauchtumspflege, der Freizeit, Kultur oder des Sports (die im Rahmen dieses Analyseschritts ausgeklammert wurden) mit keinerlei Bejahung der 5 positiven Selbstdarstellungen einher. So verneinen beispielsweise 3 islamische Vereinigungen alle in der hier relevanten Analyse berücksichtigten Items und gehen lediglich religiösen Tätigkeiten oder der Kulturpflege nach.

Wie bereits angesprochen werden die zwischen Selbstbildern und Tätigkeiten identifizierten Stimmigkeiten und Diskrepanzen im Rahmen dieser Auswertung später mit einem weiteren Untersuchungsschritt zusammengeführt: Insbesondere die Aktivitäten müssen mit dem Analysekonstrukt der ,Aufgeschlossenheit gegenüber der Umwelt“" abgeglichen werden, um adäquate Beurteilungen über die Bonding/Bridging-Ausrichtungen der MSO treffen zu können. Diese Aufgeschlossenheit - oder Abgrenzung - gegenüber anderen Personengruppen oder (Kollektiv-)Akteuren wird im nächsten Kapitel zunächst anhand der Festlegung von Mitgliedschaftsvoraussetzungen und Zielgruppen sowie des Kooperationsverhaltens ermittelt, um im Anschluss daran anhand der relevanten, schrittweise inspizierten Eigenschaften der MSO im Gesamtbild Bonding/Bridging-Typen zu identifizieren.

\subsubsection{Aufgeschlossenheit gegenüber der Umwelt: Mitgliedschaftsvoraussetzungen, Zielgruppen, Kooperationsverhalten}

\subsubsection{Erörterung zur Kategorisierung als bonding}

Aufgeschlossenheit - oder Abgrenzung - gegenüber anderen Personengruppen können anhand der Festlegung von Mitgliedschaftsvoraussetzungen und Zielgruppen sowie des Kooperationsverhaltens ermittelt werden. Diese drei Kriterien werden zusammen als „Aufgeschlossenheit gegenüber der Umwelt“ betrachtet. Sie müssen in toto in den Blick genommen werden, um profunde Aussagen über Offenheit oder Exklusivität einer MSO machen zu können. Bevor eine Deskription und Interpretation der vorzufindenden Kombinationen der drei Aspekte erfolgt 
(7.2.3.2), soll zunächst diskursiv begründet werden, welche Antworten jeweils als bonding eingestuft werden, da dies nicht selbsterklärend ist.

\section{Mitgliedschaftsvoraussetzungen}

Der Frage nach konkreten Mitgliedschaftsvoraussetzungen war die Filterfrage: „Gibt es bestimmte Voraussetzungen für die Mitgliedschaft in Ihrer Organisation?“ vorgeschaltet. Bei Bejahung waren in der darauffolgenden Frage mehrere Antworten möglich: „Empfehlungen von Mitgliedern“, ,,die Zugehörigkeit zu einer bestimmten Glaubensgemeinschaft", ,ein bestimmtes Alter", ,ein bestimmtes Geschlecht“, „ein bestimmter kultureller Hintergrund bzw. eine bestimmte Nationalität" und „Sonstiges“.

Mit der Zugehörigkeit zu einer spezifischen Glaubensgemeinschaft oder Herkunftskultur als Voraussetzung für die Mitgliedschaft in einer MSO erfolgt eine deutliche Grenzmarkierung gegenüber andersgläubigen oder fremdkulturellen Personen, für die eine Mitgliedschaft nicht vorgesehen ist. ${ }^{106}$ Die Bedingungen, ein bestimmtes Alter oder Geschlecht zu haben, sind zwar restriktive Kriterien, als sozialstrukturelle Merkmale können sie aber alle Menschengruppen betreffen und sind daher nicht per se als Indizien für eine offensichtliche Exklusivität einer MSO zu betrachten. Gleiches gilt für Empfehlungen von Mitgliedern, die sehr unterschiedliche Personengruppen betreffen können.

Die unter der offenen Kategorie ,Sonstiges“ lediglich zweimal genannte Bedingung einer bestimmten politischen Einstellung oder Anhängerschaft (von Mustafa Kemal Atatürk) ist der religiösen Orientierung insofern gleichzustellen, als es sich hier um eine auf geteilten politischen Wertorientierungen basierende Interessengemeinschaft handelt, die ihre Wurzeln im Herkunftsland haben und die nun in einem selbstorganisierten Rahmen in Deutschland gemeinsam weiter unterstützt und stark gemacht werden. An diesem Punkt könnte argumentiert werden, dass man eine

\footnotetext{
${ }^{106}$ An dieser Stelle ist anzumerken, dass im zivilgesellschaftlichen Sektor freiwilliger Selbstorganisationen das Prinzip gilt, dass sich Personen mit gleichen Weltanschauungen, Neigungen und Vorlieben zusammentun. Insofern ist zu bedenken, dass sich andere davon ggf. gar nicht ausgeschlossen fühlen, da der Wunsch nach Mitwirkung für sie ohnehin nicht relevant ist. Insbesondere in Bezug auf spezifische religiöse Vereinigungen ist daher zu hinterfragen, inwieweit eine Mitgliedschaft seitens nicht-religiöser oder andersgläubiger Personen überhaupt in Betracht gezogen wird und von Interesse ist. An anderer Stelle wurde bereits thematisiert, dass subjektive Intentionen und Motive sowie durch spezifische Organisationen vorgehaltene Gelegenheitsstrukturen und Anreize miteinander kompatibel sein sollten (II. 4.3.3.2; II. 4.4). Dies gilt insbesondere für religiöse Vereinigungen, die einer gläubigen Person das Ausleben und die Bestätigung ihres Glaubens durch die hierfür benötigte und dort gewährleistete Kommunikabilität, wie etwa die gemeinsame religiöse Praxis, ermöglichen (vgl. Pollack 2018: 38 f.; Krech 1999: 31 f.).
} 
Religionszugehörigkeit oder politische Orientierung annehmen kann. Dies ist allerdings normativ voraussetzungsreich, denn es geht um die intrinsische Akzeptanz bestimmter Einstellungen und die Befürwortung eines Weltbildes mit charakteristischen Denkweisen und Lebensauffassungen. Insbesondere mit der Festlegung einer spezifischen Religionszugehörigkeit als Mitgliedschaftsbedingung gehen stärkere ideativ-identifikative Homogenitäts- bzw. Ununterscheidbarkeitsforderungen einher, die mit Blick auf die hier relevante Analyse und die Zugehörigkeit zu einer Gemeinschaft als stärker abgrenzend einzustufen sind. Denn die Demarkationslinie wird inhaltlich konkretisiert und damit das Charakteristische der Zugehörigkeit verdeutlicht.

Vor dem Hintergrund dieser Überlegungen werden die drei Voraussetzungen der Zugehörigkeit zu einer bestimmten Glaubensgemeinschaft, ein bestimmter kultureller Hintergrund bzw. eine bestimmte Nationalität oder eine politische Einstellung als Bonding-Mitgliedschaftsvoraussetzungen kategorisiert. Die anderen ,weicheren', permissiveren Voraussetzungen (Empfehlungen von Mitgliedern; Alter; Geschlecht etc.) werden, auch wenn sie kumulieren, nicht den BondingMitgliedschaftsbedingungen zugerechnet. Wenn sie in Kombination mit den drei aufgeführten Bonding-Kriterien genannt werden, werden sie im Rahmen der Auswertung nicht mitberücksichtigt (bzw. bei spezifischer Relevanz nur im Einzelfall erwähnt). Bei Zugrundelegung dieser Kategorisierung zeigt sich, dass bei 18 Prozent der MSO Bonding-Mitgliedschaftsvoraussetzungen vorzufinden sind (Tab. 7.32).

Tab. 7.32 Kategorisierung der Mitgliedschaftsvoraussetzungen nach bonding

\begin{tabular}{lcc}
\hline & Häufigkeit & Prozent \\
\hline Kein bonding & 210 & 82,0 \\
\hline Bonding & 46 & 18,0 \\
\hline Gesamt & 256 & 100,0 \\
\hline
\end{tabular}

\section{Zielgruppen}

Die Frage, welche Gruppen die Angebote und Dienstleistungen der MSO nutzen können, war mit einer einfachen Nennung zu beantworten ${ }^{107}$. Die Antwortvorgaben, dass sie sich an Personen mit den gleichen religiösen Überzeugungen richten,

107 „Unsere Aktivitäten und Angebote richten sich nur an Mitglieder“; ,Unsere Aktivitäten und Angebote richten sich an Personen mit den gleichen religiösen Überzeugungen“; ,Unsere Aktivitäten und Angebote sind für alle offen“; ,Sonstiges“. 
wird entsprechend der Mitgliedschaftsvoraussetzung einer spezifischen Religionszugehörigkeit als bonding kategorisiert. Wenn die Mitglieder die ausschließlichen Adressaten der Aktivitäten sind, so ist dies als ein zentrales Merkmal von zivilgesellschaftlichen Selbstorganisationen einzuordnen: Menschen tun sich zusammen bzw. treten auf Basis einer bewussten Entscheidung in eine Vereinigung ein, um gemeinschaftliche Zwecke und Interessen zu verfolgen, die den eigenen Zusammenschluss und die diesem offiziell Zugehörigen betreffen. Diese charakteristische Selbstreferentialität ist damit als bonding einzuordnen, jedoch nicht automatisch mit einer Exklusivität gleichzusetzen. Denn das Mitglied-werden-Können ist ggf. nicht abhängig von spezifischen Individualmerkmalen oder normativen Orientierungen und daher als ein relativ liberales Zielgruppenkriterium einzustufen, das im Zusammenhang mit den Voraussetzungen für die Erlangung des Mitgliedschaftsstatus (s. o.) zu betrachten ist.

Unter dem Item „Sonstiges“ wurde in zwei Fällen „Menschen der Muttersprache“ sowie eine Offenheit der Angebote „nur für Frauen und Mädchen“ vermerkt, wobei ersteres als bonding eingruppiert wurde. In vier weiteren Fällen wurde angegeben, dass ein bestimmter Bereich von Angeboten nur Mitglieder oder Gleichreligiöse („Gläubige“) adressiert, andere Aktivitäten aber auch für andere Personen (,Nicht-Mitglieder“) offen sind. Diese Kompromisslösung wird mit dem Label „teils/teils“ und nicht als bonding kategorisiert. Insgesamt fallen damit 30 Zielgruppenbestimmungen (12\%) bonding aus (Tab. 7.33).

Tab.7.33 Häufigkeitsauszählungen spezifischer Zielgruppenbestimmungen

\begin{tabular}{lcc}
\hline & Häufigkeit & Prozent \\
\hline Unsere Aktivitäten und Angebote sind für alle offen. & 207 & 83,1 \\
\hline Unsere Aktivitäten und Angebote richten sich nur an Mitglieder. & 18 & 7,2 \\
\hline $\begin{array}{l}\text { Unsere Aktivitäten und Angebote richten sich an Personen mit } \\
\text { den gleichen religiösen Überzeugungen. }\end{array}$ & 11 & 4,4 \\
\hline Teils nur für Mitglieder oder Gleichreligiöse & 4 & 1,6 \\
\hline Sonstiges (Geschlecht) & 1 & 0,4 \\
\hline Sonstiges (Muttersprache) & 1 & 0,4 \\
\hline Sonstiges (Afrikabezüge; 1 x Straßentiere) & 7 & 2,8 \\
\hline Gesamt & 249 & 100,0 \\
\hline
\end{tabular}




\section{Kooperationen}

Der Frage nach konkreten Organisationen, mit denen derzeit oder in den letzten Jahren zusammengearbeitet wird bzw. wurde, ging eine Filterfrage nach überhaupt vorhandener Kooperation (in den letzten fünf Jahren) voraus. In Mehrfachantworten waren bestimmte Typen von Organisationen zu markieren, mit denen zusammengearbeitet wird oder wurde. ${ }^{108}$ Eine Aufsummierung der Kennzeichnungen zeigt, dass in einer Marge von 13 bis 20 Prozent unter den MSO am häufigsten mit 2 bis 5 Organisationstypen zusammengearbeitet wird (Tab. 7.34).

Tab. 7.34 Anzahl der Kooperationspartnertypen gesamt

\begin{tabular}{ccc}
\hline Anzahl & Häufigkeit & Prozent \\
\hline 1 & 15 & 7,7 \\
\hline 2 & 39 & 19,9 \\
\hline 3 & 30 & 15,3 \\
\hline 4 & 26 & 13,3 \\
\hline 5 & 32 & 16,3 \\
\hline 6 & 15 & 7,7 \\
\hline 7 & 13 & 6,6 \\
\hline 8 & 9 & 4,6 \\
\hline 9 & 6 & 3,1 \\
\hline 10 & 7 & 3,6 \\
\hline 11 & 1 & 0,5 \\
\hline 12 & 2 & 1,0 \\
\hline 13 & 1 & 0,5 \\
\hline Gesamt & 196 & 100,0 \\
\hline
\end{tabular}

Die Zusammenarbeit mit anderen Organisationen ist ein Indikator für die Offenheit und den Austausch der MSO mit anderen, auf unterschiedlichen gesellschaftlichen Ebenen angesiedelten Organisationen. Hier ist davon auszugehen, dass eine große Anzahl von verschiedenen Organisationen, mit denen zusammengearbeitet wird, eine größere Offenheit der MSO anzeigt, als wenn lediglich ein

\footnotetext{
${ }^{108}$ Festgestellt wird dadurch also nicht die genaue Anzahl der Kooperationspartner insgesamt, sondern die Anzahl der Organisationsarten, mit denen zusammengearbeitet wird (7.1.6).
} 
Kooperationspartnertypus genannt wird. Bei diesem ist dann auch von besonderem Interesse, um welchen es sich im konkreten Fall handelt, weil darüber auf eine Bridging- oder Bonding-Ausrichtung geschlossen werden kann, wenn beispielsweise eine christliche Vereinigung in zwei Fällen ausschließlich mit der Kirche oder mit dem christlichen Hilfswerk zusammenarbeitet.

\subsubsection{Gruppierungen und ihre Diskussion}

Im Folgenden werden die Kombinationen von Mitgliedschaftsvoraussetzungen und Zielgruppen der Aktivitäten betrachtet und diskutiert. Außen vor gelassen wird dabei das Kooperationsverhalten, das - wie oben thematisiert - je nach Ausmaß die Aufgeschlossenheit einer Organisation potenziert oder abschwächt. Eine detaillierte Auflistung der den Gruppen zugehörigen Einzelfälle findet sich in einer separaten Übersicht im elektronischen Zusatzmaterial (Tab. A $18^{109}$ ).

Gruppe 1: Keine Mitgliedschaftsvoraussetzungen / Angebote für alle offen $(68,9 \%)$

Wenn hinsichtlich der Mitgliedschaft keine Voraussetzungen bestehen und mit den Aktivitäten diverse Personengruppen adressiert werden, kann von einer grundsätzlichen Aufgeschlossenheit der MSO gesprochen werden. Die Konstellation verweist auf ihre Bereitschaft, unterschiedliche Menschen zu involvieren und sich in der Gruppe mit verschiedenen Anschauungen auseinanderzusetzen. Vonseiten aller MSO werden in der Mehrzahl weder Mitgliedschaftsvoraussetzungen noch Zielgruppen festgelegt. Dabei fällt die Zugänglichkeit der säkularen MSO um rund 15 Prozentpunkte höher aus als bei den religiösen (Abb. 7.32).

Gruppe 2: Keine Mitgliedschaftsvoraussetzungen, aber eine spezifische Zielgruppe $(\mathbf{7 , 8} \%)$

Wenn grundsätzlich eine Offenheit für die Mitgliedschaft besteht, die Angebote und Dienstleistungen aber nur für eine spezifische Zielgruppe vorgesehen sind, dann kann davon ausgegangen werden, dass sich bonding und bridging potenziell

${ }^{109}$ Zum Umgang mit fehlenden Werten: Fehlende Werte wurden hier gesondert ausgewiesen und können nicht unterschlagen werden, da nicht gewusst werden kann, warum die Funktionsträger in 15 Fällen hinsichtlich der Zielgruppenfrage (und in einem Fall hinsichtlich der Mitgliedschaftsvoraussetzungen) keine Angaben gemacht haben (multinominal codierte Variablen). Wenn die fehlenden Werte unbeachtet bleiben, können damit hinsichtlich der Eingruppierung der MSO fehlerhafte Interpretationen einhergehen. 
ausbalancieren (können). Denn Kommunikation und Interaktion finden einerseits innerhalb der eigenen Gemeinschaft statt, andererseits zeigt sich die Gruppierung für die Mitwirkung und Teilnahme von fremden, außerhalb der Gemeinschaft stehenden Menschen prinzipiell offen und formuliert keine Zugangsbeschränkungen. Diese Konstellation ist mit 7 bis 8 Prozent auf einem gleichen Niveau für alle MSO und ohne nennenswerte Unterschiede zwischen religiösen und säkularen auszumachen (Abb. 7.32).

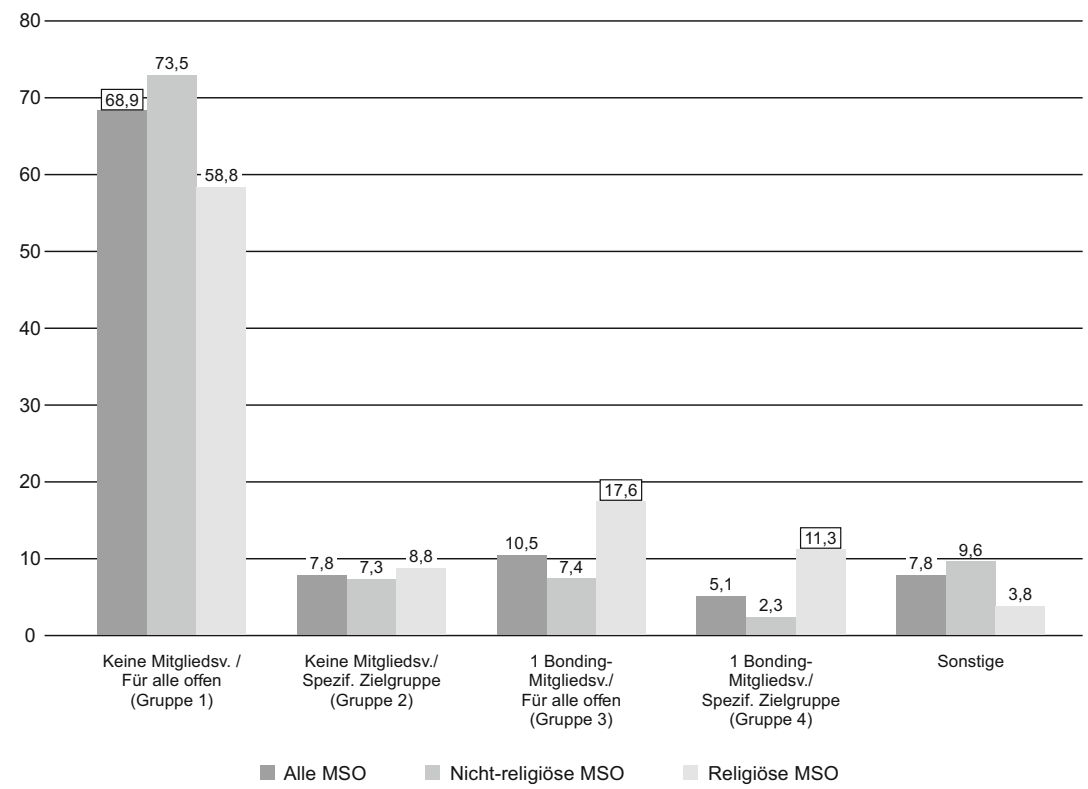

$n=257$

Abb. 7.32 Kombinationen von Mitgliedschaftsvoraussetzungen und Zielgruppen (in \%)

Allerdings erscheint es paradox, wenn sich die Aktivitäten in 5 sich einer religiösen Tradition zuordnenden MSO ausschließlich an Personen mit den gleichen religiösen Überzeugungen richten, die Zugehörigkeit zu einer bestimmten Glaubensgemeinschaft jedoch keine Voraussetzung für die Mitgliedschaft ist (Tab. A $18 \mathrm{im}$ elektronischen Zusatzmaterial). Dies kann zum einen dahingehend interpretiert werden, dass die Aktivitäten als für andere nicht interessant betrachtet werden; zum anderen könnte es sein, dass sich die betreffenden religiösen 
Vereinigungen als aufgeschlossen und gegenüber Andersgläubigen als tolerant präsentieren möchten, über die Festlegung eines spezifischen Adressatenkreises aber ein subtiler Exklusionsmechanismus wirkt.

Gruppe 3: Eine Bonding-Mitgliedschaftsvoraussetzung, aber Angebote für alle offen $(10,5 \%)$

Wenn eine bestimmte religiöse Orientierung oder ein spezifischer kultureller bzw. nationaler Herkunftshintergrund eine Bedingung für die Mitgliedschaft ist, die Angebote aber grundsätzlich für alle zugänglich sind, dann können soziale Beziehungen und Bindungen potenziell auch außerhalb der betreffenden MSO aufgebaut und aufrechterhalten werden. Innerhalb dieser Gruppe sind zwischen säkularen und religiösen MSO Unterschiede erkennbar (Differenz: 10 Prozentpunkte), die darauf zurückzuführen sind, dass die Möglichkeit einer Mitgliedschaft an die Bedingung der Zugehörigkeit zu einer bestimmten Glaubensgemeinschaft geknüpft wird.

$\mathrm{Zu}$ hinterfragen ist, inwieweit Brücken $\mathrm{zu}$ anderen Personengruppen tatsächlich bestehen bzw. geschlagen werden. Denn die religiöse bzw. kulturelle Zugehörigkeit wird das Miteinander in der MSO und auch die Ausrichtung der Aktivitäten höchstwahrscheinlich mitbestimmen und daher auch insbesondere Personen mit gleichen Konfessionen und kulturellen Hintergründen attrahieren. Dieses wird umso mehr zutreffen, wenn - wie im Fall einer säkularen MSO - über doppelte Mitgliedschaftsbedingungen verfügt wird. Die religiösen, kulturellen oder politischen Wertorientierungen und Verortungen der MSO werden somit mit großer Wahrscheinlichkeit ausschließlich bestimmten Personengruppen vorbehalten sein. Dies wird auch dann zutreffen, wenn sich die MSO gegenüber anderen, fremden Menschen aufgeschlossen zeigen, da sich diese nicht für die in Rede stehenden MSO interessieren und sich von ihren Denkrichtungen und Tätigkeiten nicht angesprochen fühlen. Dann kann eine betreffende MSO als sich gesellschaftlich abschließend oder abgrenzend wahrgenommen werden, auch wenn sie sich nicht als eine solche versteht.

Gruppe 4: Eine Bonding-Mitgliedschaftsvoraussetzung / eine spezifische Zielgruppe $(\mathbf{5 , 1} \%)$

Die Festlegung auf eine Bonding-Mitgliedschaftsvoraussetzung sowie auf eine spezifische Zielgruppe weist hingegen auf das Bedürfnis einer MSO hin, sich gegenüber anderen Personenkreisen und Gruppierungen abzugrenzen und mit ihren Zielen und Angeboten selbstreferentiell und exklusiv zu sein bzw. zu agieren. Auch in dieser Gruppe treten die religiösen MSO in Bezug auf Festlegungen 
hervor (11,3\% vs. 2,3\%). Wird in dieser Formierung darüber hinaus nicht mit anderen Organisationen zusammengearbeitet, ist davon auszugehen, dass lediglich der Zusammenhalt innerhalb der eigenen Gemeinschaft gefördert und gruppenintern bindendes Sozialkapital produziert wird. Diese Konstellation ist lediglich für 3 MSO festzustellen. Das Abgrenzungsbedürfnis zeigt sich verstärkt, wenn zwei Bonding-Mitgliedschaftsvoraussetzungen festgelegt wurden, was in dieser Gruppe jedoch nur bei einem Fall vorkommt (Tab. A 18).

Theoretisch kann argumentiert werden, dass eine grundlegende Voraussetzung jeder Gruppen- bzw. Organisationsbildung eine Abgrenzung nach außen ist (Neidhardt 2017/[1979]: 436 ff.; Tyrell 1983: 82 f.; Aldrich/Ruef 2006: 4). Formation, Konsolidierung und Stabilisierung der Gruppe finden im Binnenraum der MSO statt und sie sind verknüpft mit Grenzziehungen nach außen. Identität und Kohäsion der Gruppe werden durch die Abgrenzung gegenüber Nicht-Mitgliedern respektive Nicht-Religiösen und der Festlegung bestimmter Zielgruppen hergestellt. Damit wird ein Innen-Außen-Verhältnis konstituiert, das als eine wesentliche Bedingung für Definition, Bestand und Erhalt der Organisation $\mathrm{zu}$ betrachten ist (Luhmann 2005a/[1975a]: 12 f.). Dies impliziert, dass Solidarisierung, Gruppendenken und Zusammengehörigkeitsgefühl (WirBildung) in der Organisationsinnenwelt mit erwarteten Loyalitäten und Formen der Gegenseitigkeit verknüpft ist (II. 4.2.4 und 4.2.5).

\subsubsection{Quervergleich zwischen den MSO: bridging oder bonding? - Muster und Typenbildung}

Um weitere Muster erkennen und Typen bilden zu können, bedarf es im Folgenden einer genauen Inaugenscheinnahme, wie sich die spezifischen Aktivitätsspektren der MSO mit der Analysedimension ,Aufgeschlossenheit gegenüber der Umwelt“ (7.2.3) zusammenfügen. Überprüft wird nun, inwieweit die Nutzung der Angebote bzw. die Teilnahme an den Aktivitäten durch die Festlegung bestimmter Zielgruppen eingeschränkt und inwieweit diesbezüglich vorhandene Eingrenzungen weiterhin durch die Existenz bestimmter Mitgliedschaftsvoraussetzungen verstärkt werden. Wenn sowohl spezifische Zielgruppen als auch Mitgliedschaftsbedingungen bestimmt worden sind, wird des Weiteren geprüft, ob und in welchem Ausmaß die MSO Kooperationen eingehen und welche Einrichtungen das betrifft. 
Insgesamt verdeutlicht die zwecks Typenbildung vollzogene Untersuchung, dass mit der Priorisierung der Binnenorientierung einhergehende deutliche Grenzmarkierungen mit Umweltbeziehungen zusammen auftreten können: Selbstbezüglichkeiten und Abgrenzungstendenzen von Organisationen treten in der Mehrzahl nicht ausschließlich und eindeutig in Erscheinung, sondern gehen mit nach außen gerichteten Aktivitäten und Kooperationen einher. Wenn MSO, deren Aktivitäten nur für die eigenen Mitglieder bzw. Gleichreligiöse vorgesehen sind, durch Kooperationen und Bridging-Aktivitäten zugleich eine starke Außenorientierung aufweisen und sich das Handeln somit auch auf andere gesellschaftliche Gruppen und Akteure bezieht, dann wird im Rahmen vorliegender Analyse von einer Bonding/Bridging-Performanz gesprochen.

Eine Einordnung in entweder Bridging- oder Bonding-Ausrichtungen kann in der Realität folglich zumeist nicht eindeutig und trennscharf erfolgen (7.2; 7.2.2). Stattdessen ist von unterschiedlichen Intensitätsgraden von bridging und bonding und variierenden Mischungsverhältnissen auszugehen. Um dem multidimensionalen Phänomen gerecht zu werden, ist eine detaillierte Analyse unabdingbar. Bei dieser wurde der Fokus zur Erhöhung der Trennschärfe „ex positivo“ zunächst auf das Vorhandensein von Bridging- und sozialpädagogischen Aktivitäten gelegt, die kontinuierlich separat betrachtet wurden. Wenn sich keiner der beiden Bereiche als relevant zeigte, wurde geprüft, welchen anderen Aktivitäten die MSO nachgehen. Mit dieser Analysestrategie wird also die Prävalenz spezifischer Aktivitäten festgestellt und auf dieser Grundlage werden aussagekräftige Typen gebildet; nicht ermittelt wird, wie viel Bonding-Aktivitäten in Bridging-Typen stecken. Bonding-Charakteristika sind bei der Typenbildung somit insbesondere in Hinblick auf die Bestimmung von Zielgruppen, Mitgliedschaftsvoraussetzungen sowie das Kooperationsverhalten eingeflossen (Analysedimension „Aufgeschlossenheit gegenüber der Umwelt"). Dieses zur Kenntnis zu nehmen ist wichtig für die Einordnung der Typenbildung und der auf ihrer Basis im Weiteren ermittelten Ergebnisse in den Gesamtkontext der Bonding/Bridging-Auswertung, in der methodisch unterschiedliche Analyseverfahren angewendet wurden.

Die verschiedenen Mischformen bzw. Muster wurden in 8 Typen überführt (Übersicht 7.1; $\mathrm{n}=246 ; \mathrm{n}_{\mathrm{S}}=167 ; \mathrm{n}_{\mathrm{R}}=79$ ). Davon lassen sich 4 als bridging, 3 als bonding sowie einer als bonding/bridging charakterisieren. Sie werden mit ihren prozentualen Anteilen sowie Verteilungen auf religiöse Vereinigungen in der Übersicht 7.1 dargestellt. ${ }^{110}$ Es zeigt sich, dass über die Hälfte der MSO (54\%) sehr aufgeschlossen und kontaktfreudig ist (Typ Bridging I). Die zur

${ }^{110}$ Anmerkung zum Auswertungsverfahren: An dieser Stelle ist nochmals zu explizieren, dass im Rahmen der Analyse unterschiedliche Auswertungsverfahren kombiniert wurden. Als Ergänzung zur quantitativen Datenerhebung wurde eine Auswertungsstrategie 
Übersicht 7.1 Muster von bridging und bonding in 8 Typen

$$
\text { Bridging-Typen (I-IV) }
$$

$$
\text { Typ Bridging I = „Die Extravertierten“ }
$$

Die große Mehrzahl der befragten MSO (54\%) übernimmt Bridging-Aktivitäten, kooperiert mit anderen Einrichtungen und zeigt sich hinsichtlich der Mitwirkung in den Vereinigungen und der Teilnahme an ihren Tätigkeiten und Angeboten offen.

Merkmale: keine Festlegung von Mitgliedschaftsvoraussetzungen und Zielgruppen; Kooperationen; Bridging-Aktivitäten (in der großen Mehrzahl in Kombination mit Tätigkeiten der Bildung und Sozialarbeit)

Anzahl MSO gesamt: 133 (54\%) Darunter 34 religiöse (43\% der RMO):

22 islamische | 7 alevitische | 4 christliche (2 orthod., 1 ev.freik., 1 konfess. gemischt) | 1 ezidische

Typ Bridging II = „Die nicht-kooperierenden Aufgeschlossenen“

Dieser Typus ist im Grunde deckungsgleich mit dem zuvor beschriebenen.

Die MSO (10\%) kooperieren allerdings nicht mit anderen Organisationen.

Merkmale: keine Festlegung von Mitgliedschaftsvoraussetzungen und Zielgruppen; keine Kooperationen; Bridging-Aktivitäten (in der großen Mehrzahl in Kombination mit Tätigkeiten der Bildung und Sozialarbeit)

Anzahl MSO gesamt: $25(\mathbf{1 0 , 2} \%)$ Darunter 8 religiöse $(10,1 \%$ der RMO)

5 islamische | 1 alevitische | 1 christliche (1 ev.-freik.) |

1 ezidische

Typ Bridging III = „Die offenen sozialpädagogisch Tätigen“

Die diesem Typus zugeordneten MSO (5\%) gehen erzieherischen und/oder sozialarbeiterischen Aktivitäten nach. Bridging-Aktivitäten lassen sich explizit nicht nachweisen. Da die betreffenden Organisationen weder Zielgruppen noch Mitgliedschaftsvoraussetzungen festlegen und darüber hinaus mehrheitlich mit anderen Einrichtungen zusammenarbeiten, sind sie als bridging agierend einzuordnen.

Merkmale: keine Festlegung von Mitgliedschaftsvoraussetzungen und Zielgruppen; mehrheitlich Kooperationen; erzieherische und/oder sozialarbeiterische Aktivitäten

Anzahl MSO gesamt: $11(4,5 \%) \quad$ Darunter 1 religiöse (islamische)

Typ Bridging IV = „Die mitgliedschaftsbewussten Außenorientierten“

Vorhandene Mitgliedschaftsvoraussetzungen werden nicht von Zielgruppenbestimmungen flankiert.

In lediglich 3 Fällen wird eine Zielgruppenbestimmung (der Mitglieder bzw. Religiösen) nur teilweise geltend gemacht* ${ }^{\star}$. Die MSO kooperieren mit anderen Einrichtungen und verfolgen zumeist mehrere Bridging-Aktivitäten sowie Tätigkeiten der Bildung und Sozialarbeit.

Merkmale: Festlegung von Mitgliedschaftsvoraussetzungen; keine Zielgruppenbestimmung; Kooperationen; viele Bridging-Aktivitäten (bis auf einen Fall in Kombination mit Tätigkeiten der Bildung und Sozialarbeit)

Anzahl MSO gesamt: 30 (12,2\%) Darunter 13 religiöse (16,5\% der RMO):

[Typen-ID 1-30] 5 christliche (4 ev.-freik.; 1 syrisch-orthod.) | 4 islamische | 1 alevitische | 2 jüdische | 1 ezidische

* Ein bestimmter Bereich von Angeboten ist nur für Mitglieder oder Gleichreligiöse (,Gläubige“) bestimmt, andere Aktivitäten sind aber auch für andere Personen (,Nicht-Mitglieder“) offen. 
Übersicht 7.1 (Fortsetzung)

Bonding-Typen (I-III)

$$
\text { Typ Bonding I = „Die Selbstbezogenen“ }
$$

Erkennbar ist eine Gruppenbezogenheit, die charakterisiert wird durch die doppelte Bestimmung von Personenkreisen, denen die Mitwirkung in den Organisationen und die sozialarbeiterischen (und einzelnen brückenbauenden) Aktivitäten der MSO vorbehalten sind. Ein weiteres Merkmal ist die Nicht-Kooperation mit anderen Einrichtungen. Die Konstellationen weisen auf die Dominanz einer Bonding-Performanz hin.

Merkmale: Festlegung von Mitgliedschaftsvoraussetzungen UND Zielgruppen; keine Kooperationen

$\frac{\text { Anzahl MSO gesamt: }}{\text { [Typen-ID 31-33] }}$ (1,2\%) $\quad \frac{\text { Darunter } 2 \text { religiöse: } 1 \text { islamische | } 1 \text { christliche (griech.- }}{\text { orthod.) }}$

Typ Bonding II = „Die offeneren Selbstbezogenen“

Erkennbar ist eine Gruppenbezogenheit, die charakterisiert ist durch die Festlegung von Mitgliedschaftsvoraussetzungen oder Zielgruppen sowie die mehrheitliche Nicht-Existenz von Kooperationen. Es überwiegen erzieherische bzw. sozialarbeiterische Tätigkeiten, die in 10 Fällen von ein bis zwei Bridging-Aktivitäten flankiert werden. Die in einzelnen Fällen vorhandene potenzielle Aufgeschlossenheit wird die Selbstbezogenheit der betreffenden MSO höchstwahrscheinlich nicht relativieren.
Anzahl MSO gesamt: $15(6,1 \%)$
Darunter 6 religiöse $(7,6 \%$ der RMO):
[Typen-ID 34-48]
3 christliche ( 1 kath., 1 ev., 1 ev.-freik.) | 2 islamische |
1 alevitische

\section{[ $\rightarrow$ Binnenvarianzen des Typus Bonding II]}

a) Merkmale: Festlegung von Zielgruppen; keine Kooperationen

Die Zielgruppenbestimmung der Mitglieder, die Bereiche der Aktivitäten und das Nicht-Vorhandensein von Kooperationen weisen in den spezifischen Konstellationen auf die Prävalenz von Bonding-Performanzen hin.

Anzahl MSO gesamt: 8

Darunter 1 religiöse: 1 islamische

b) Merkmale: (z. T. geltende) Festlegung von Zielgruppen; Kooperationen

Erkennbar ist eine Gruppenbezogenheit, die charakterisiert wird durch die (teilweise geltende) Zielgruppenbestimmung der Mitglieder, denen die Aktivitäten der MSO vorbehalten sind. Die Kooperation mit maximal zwei Einrichtungen deutet auf Aufgeschlossenheit hin, wird die Selbstbezogenheit der betreffenden MSO aber nicht relativieren. Die Konstellationen lassen auf Bonding-Ausrichtungen schließen.

Anzahl MSO gesamt: 3

Darunter 2 religiöse: 1 alevitische | 1 christliche

c) Merkmale: Festlegung von Mitgliedschaftsvoraussetzungen; mehrheitlich keine Kooperationen

Die Gruppenbezogenheit ist charakterisiert durch die Mitgliedschaftsbedingung der Zugehörigkeit zu einer Glaubensgemeinschaft. Die Nutzung der Aktivitäten ist prinzipiell offen für andere Personenkreise. Die Glaubenszugehörigkeit wird das Miteinander in der MSO mitbestimmen und die Aktivitäten höchstwahrscheinlich Gleichreligiöse attrahieren. Darüber hinaus wird überwiegend nicht mit anderen Einrichtungen kooperiert. Es ist davon auszugehen, dass die betreffenden MSO bonding agieren.

Anzahl MSO gesamt: $4 \quad$ Darunter 3 religiöse: 2 christliche | 1 islamische 
Übersicht 7.1 (Fortsetzung)

Typ Bonding III = „Die eigeninteressengeleiteten Kultur- und Freizeitvereine“
Die Mitglieder gehen spezifischen Eigeninteressen (Kultur, Freizeit, Sport, Religion) nach und
agieren daher gruppenbezogen für und unter sich. Offentlichkeitsbezüge und Kooperationen
sind für sie überwiegend nicht relevant. Auch bei diesen MSO lässt sich ein zentrales Merkmal
von zivilgesellschaftlichen Selbstorganisationen erkennen: Menschen tun sich zusammen bzw.
treten in eine Vereinigung ein, um gemeinschaftliche Zwecke und Interessen zu verfolgen, die
den eigenen Zusammenschluss betreffen. Wenngleich sich die Beteiligten i. d. R. gegenüber
ihrer Umwelt offen zeigen, agieren sie aufgrund der charakteristischen interessengeleiteten
Konzentration auf die eigene Gruppe und ihre Aktivitäten bonding.

Merkmale: i.d.R keine Festlegung von Mitgliedschaftsvoraussetzungen und Zielgruppen; keine oder sehr wenige Kooperationen

Anzahl MSO gesamt: 12 (4,9\%) Darunter 4 religiöse: 3 islamische | 1 alevitische [Typen-ID 49-60]

Bonding/Bridging-Typen $=$ „Die (hoch-)aktiven Brückenbauer“

Die durch die Bestimmung von Zielgruppen und/oder Mitgliedschaftsbedingungen einfach oder zweifach exklusive Formierung und Gruppenorientierung der MSO geht mit zumeist äußerst vielfältigen Kooperationsbeziehungen und einem breiten, dominant bridging-orientierten Aktivitätsspektrum einher.

Die Konzentration auf die Gruppenmitglieder fällt mit einer (stark ausgeprägten) Außenorientierung und hohen Aktivitätsraten zusammen. Dies indiziert die Verflechtung von Bonding- und Bridging-Ausrichtungen.

Durch brückenschlagende Tätigkeiten und die Zusammenarbeit mit für die strukturelle Integration relevanten Einrichtungen werden die Belange und Interessen der Gruppe an das Umfeld angedockt und dort thematisiert.

2 der MSO sind als Prototypen außenorientierter Selbsthilfeorganisationen einzustufen. Sie adressieren Frauen und Mädchen oder Menschen mit dem gleichen kulturellen Hintergrund.

Merkmale: mehrheitlich Festlegung sowohl von Mitgliedschaftsvoraussetzungen UND Zielgruppen (im Gegensatz zum Typ Bridging IV); vielfältige Kooperationsbeziehungen; zumeist hochaktiv = viele Bridging-Aktivitäten

Anzahl MSO gesamt: $17(6,9 \%)$ [Typen-ID 61-77]

Darunter 11 religiöse (13,9\% der RMO):

5 islamische | 3 christliche (russ.-orthod., griech.-orthod., evangelisch) | 1 alevitische | 1 jüdische | 1 hinduistische

gewählt, mit der weiterreichende Erkenntnisse gewonnen werden können als es mit einem technisch-mathematischen Zugriff allein möglich wäre. Mit einer qualitativ-interpretativen Vorgehensweise wurde die Kombination der analyserelevanten Variablen in ihrer Bedeutung für Bonding- und Bridging-Profile überprüft und eine Typologisierung der Einzelfälle vorgenommen. Es handelt sich um einen forschungspragmatischen Ansatz, mit dem die Erkenntnismöglichkeiten statistischer Analysen durch eine komplementäre, qualitativ ausgerichtete Auswertung erweitert werden. Anhand der Ermittlung von Häufigkeiten, mit denen bestimmte Kategorien besetzt sind, konnten die Ergebnisse in nach ihren jeweiligen Merkmalen beschreibbaren Typen überführt und damit wiederum verallgemeinert und quantifiziert 
Typenbildung herangezogenen Charakteristika der MSO wurden bereits diskutiert. Im Anhang I im elektronischen Zusatzmaterial sind die Einzelfälle detailliert aufgeführt. ${ }^{111}$

Die regelmäßig geltend gemachte Bonding-,Anfälligkeit' religiöser Vereinigungen lässt sich mit der qualitativ ausgerichteten Analyse nicht belegen. Die Mehrzahl zeigt sich gegenüber der Umwelt (sehr) aufgeschlossen. In der Summe zeigen sich die säkularen MSO als mehr bridging (MO: $85 \%$ vs. RMO: $71 \%$ ), die religiösen hingegen als mehr bonding/bridging und hochaktiv (Tab. 7.35). Insofern balancieren sich Unterschiede aus. Zudem lassen sich hinsichtlich der einzelnen Konfessionen keine spezifischen Typen-Affinitäten ausmachen. Die religiösen Gemeinschaften verteilen sich auf alle Typen und lassen keine dualistischen Muster erkennen.

$$
* * *
$$

Überprüfung von (In-)Kommensurabilitäten II: Selbstdarstellung und Aktivitäten versus Aufgeschlossenheit gegenüber der Umwelt

Wie am Ende des Abschnitts 7.2.2 angekündigt soll nun in einem zweiten Gang überprüft werden, inwieweit die Aktivitäten, die Selbstdarstellung sowie die sich aus den Mitgliedschaftsvoraussetzungen, Zielgruppen und dem Kooperationsverhalten zusammensetzende Dimension „Aufgeschlossenheit gegenüber der Umwelt" bei den identifizierten Bonding-Typen (I - III) ggf. in Widerspruch zueinander stehen.

Legt man das Ziel „Etwas für das allgemeine Wohl in Deutschland zu tun“ auf die Waagschale, geraten 4 MSO in den Blick ${ }^{112}$. In zwei von diesen richten sich

werden. Die Autorin ist sich darüber bewusst, dass die Geltungsreichweite der herausgearbeiteten Sozialkapital-Typen in der Lebenswirklichkeit der MSO bestenfalls anhand qualitativer Interviews überprüft werden sollte. Ein solches Vorhaben muss allerdings einem separaten Forschungsprojekt vorbehalten bleiben.

${ }^{111}$ Der Umgang mit fehlenden Werten (Aktivitäten; Mitgliedschaftsvoraussetzungen; Zielgruppen) ist different. Bei den Aktivitäten (vierstufige Ordinalskala) werden sie als Residualkategorie behandelt: Es wird davon ausgegangen, dass Items in den betreffenden Fällen angekreuzt worden wären, wenn ihre Bejahung für die organisationale Performanz ausschlaggebend und wichtig ist. (Diesbezüglich ließen sich bei der 21 Aktivitäten umfassenden Itembatterie im Antwortverhalten auch Muster feststellen.) Hingegen werden 11 Fälle, die in Bezug auf Zielgruppen oder Mitgliedschaftsvoraussetzungen fehlende Angaben betreffen (multinominale Codierung der Antworten), aus der Analyse ausgeschlossen, da sie eine Typenzuordnung verhindern. (In 5 weiteren Fällen kann sie trotz fehlender Werte vorgenommen werden: Typen-ID 28, 45, 55, 56, 77, s. Anhang I im elektronischen Zusatzmaterial.)

112 Siehe für den Nachvollzug Anhang I im elektronischen Zusatzmaterial: Typen-ID 31, 37, 42, 43. 
Tab. 7.35 Verteilung der säkularen und religiösen MSO auf die Bridging- und BondingTypen

\begin{tabular}{|c|c|}
\hline Säkulare & Religiöse \\
\hline \multicolumn{2}{|c|}{ Bridging I } \\
\hline $59 \%$ & $43 \%$ \\
\hline & $\begin{array}{l}22 \text { islamische | } 7 \text { alevitische | } 4 \text { christliche } \\
\text { ( } 2 \text { orthod., } 1 \text { ev.-freik., } 1 \text { gemischt) | } 1 \text { ezidische }\end{array}$ \\
\hline \multicolumn{2}{|c|}{ Bridging II-IV } \\
\hline \multirow[t]{2}{*}{$26 \%$} & $28 \%$ \\
\hline & $\begin{array}{l}10 \text { islamische | } 2 \text { alevitische | } 6 \text { christliche } \\
\text { (5 ev.-freik.; } 1 \text { syrisch-orthod.) | } 2 \text { ezidische | } \\
2 \text { jüdische }\end{array}$ \\
\hline \multicolumn{2}{|c|}{ Bonding I-III } \\
\hline \multirow[t]{2}{*}{$11 \%$} & $15 \%$ \\
\hline & $\begin{array}{l}6 \text { islamische | } 4 \text { christliche (1 griech.-orthod.; } \\
1 \text { kath., } 1 \text { ev., } 1 \text { ev.-freik.) | } 2 \text { alevitische }\end{array}$ \\
\hline \multicolumn{2}{|c|}{ Bonding/Bridging } \\
\hline \multirow[t]{2}{*}{$4 \%$} & $14 \%$ \\
\hline & $\begin{array}{l}5 \text { islamische | } 3 \text { christliche (russ.-orthod., } \\
\text { griech.-orthod., evangelisch) | } 1 \text { alevitische | } \\
1 \text { jüdische | } 1 \text { hinduistische }\end{array}$ \\
\hline
\end{tabular}

die Aktivitäten nur an die Mitglieder und es wird nicht mit anderen Einrichtungen zusammengearbeitet (ID 31,37). Eine tatsächlich angestrebte Allgemeinwohlorientierung und ein Brückenbauen in der Praxis sind daher anzuzweifeln, da die Tätigkeiten kaum die Grenzen der eigenen Gemeinschaft überschreiten und anderen Gesellschaftsmitgliedern von Nutzen sein werden. Dies gilt auch für zwei weitere MSO (ID 42, 43), deren Aktivitäten sich ausschließlich an Gleichreligiöse richten und bei einer von ihnen mit der Arbeitsmarktintegration (und Selbsthilfe als zweite Tätigkeit) sehr spezifisch ausfallen. Die vorhandenen Kooperationen mit wenigen bzw. den MSO nahestehenden Organisationen werden diese Selbstbezogenheit nicht relativieren; andererseits könnte jedoch auch argumentiert werden, dass die Eingliederung der religiösen Mitglieder in den Arbeitsmarkt im Endeffekt auch der gesamten Gesellschaft zugutekommt. An dieser Stelle zeigt sich ganz deutlich, was für eine Gratwanderung es ist, ohne Kenntnis der einzelnen MSO und ihrer Performanzen in der Praxis eine Beurteilung zu (In-)Kommensurabilitäten vorzunehmen. 
Die bereits thematisierten 7 in sich potenziell widersprüchlichen Fälle wurden unter der zusätzlich erfolgten Berücksichtigung der Analysedimension ,Aufgeschlossenheit gegenüber der Umwelt“ dem Bonding III-Typus (,Die eigeninteressengeleiteten Kultur- und Freizeitvereine") zugeordnet (ID 49-60). Bei ihnen sind größtenteils keine Kooperationen und eine Offenheit für die Teilnahme an den Aktivitäten zu registrieren.

Für 3 der 7 MSO sind Inkonsistenzen festzustellen (ID 53, 55, 57). Sie verfolgen kulturelle und freizeitliche Aktivitäten, die zusammen mit der Festlegung der Zielgruppe der Mitglieder oder Mitgliedschaftsvoraussetzungen jedoch nicht die Realisierung des Ziels der Allgemeinwohlorientierung oder von anderen integrationsbezogenen Selbstdefinitionen anzeigen. Daher lässt sich schlussfolgern, dass sich das positive Selbstbild in der Praxis nicht realisieren wird. Insofern sind bei den drei betreffenden MSO im Antwortverhalten „Overstatements“ auszumachen.

Alles in allem bleibt auch nach dieser zweiten Prüfung festzuhalten, dass nur wenige Unstimmigkeiten zutage treten: Konkret können auf Basis der zweischrittigen Analyse lediglich 7 Fälle als in sich widersprüchlich ausgemacht werden. Die MSO wissen sich hinsichtlich ihrer Performanzen also selbst gut einzuschätzen.

\subsubsection{Analyse von Begründungszusammenhängen}

Entsprechend der zu Beginn dieses Kapitels aufgeführten und zu beantwortenden Fragen ist ein weiteres zentrales Anliegen, hinsichtlich der identifizierten Sozialkapitalformierungen auf „Ursachenforschung“ zu gehen und relevante Zusammenhänge aufzuzeigen. Daher werden im Folgenden die als Bonding- oder Bridging- sowie Bonding/Bridging-Typen charakterisierten Einzelfälle mit Blick auf spezifische organisationale Eigenschaften (als Prädiktor- bzw. unabhängige Variablen) näher beleuchtet. Konkret geht es um die finanziellen und personellen Ressourcenausstattungen, das Alter der Organisationen sowie homogene Mitglieder- und Engagiertenstrukturen ${ }^{113}$. Darüber hinaus wird untersucht, inwieweit sich die betreffenden MSO Fremdenfeindlichkeit, negativen öffentlichen Meinungen oder mangelhafter Einbindung seitens der Stadt ausgesetzt sehen.

\footnotetext{
${ }^{113}$ Personengruppen, Konfessionszugehörigkeit, Herkunftsländer. An dieser Stelle ist darauf hinzuweisen, dass die sozialstrukturellen Merkmale (Alter, Geschlecht, Bildungsniveau, Beruf, Einkommen) aufgrund des Erhebungsdesigns unberücksichtigt bleiben.
} 
In einem ersten Schritt werden zunächst einige der ermittelten SozialkapitalTypen zusammen mit den Prädiktorvariablen in einer Tabelle mit 16 Kopfgruppen unter die Lupe genommen und das Erkennbare beschrieben (vgl. Anhang II im elektronischen Zusatzmaterial). In einem zweiten, methodisch deutlich anders zu verortenden Schritt wird dann mit multivariaten strukturprüfenden Verfahren multiplen Regressionsanalysen - kontrolliert, inwieweit zwischen unabhängigen und abhängigen Variablen, die sowohl Bridging- als auch Bonding-Ausprägungen betreffen, Zusammenhänge festzustellen sind. Die Beschreibung der mit den Regressionen verknüpften Vorgehensweise erfolgt im weiteren Verlauf dieses Kapitels.

\subsubsection{Deskriptives Analyseverfahren auf Basis der Typenbildung}

Hinsichtlich der Eigenschaften der $\mathbf{3 0}$ als bonding (Typen I - III) kategorisierten Einzelfälle zeigen sich Gemeinsamkeiten, aber v. a. auch Varianzen in den Merkmalskombinationen (vgl. Anhang II im elektronischen Zusatzmaterial).

Zunächst springt ins Auge, dass das Alter der Organisationen sehr durchmischt ausfällt. $\mathrm{Zu}$ recht ähnlichen Anteilen sind sowohl jüngere als auch ältere MSO unter den Bonding-Typen vertreten ${ }^{114}$. Des Weiteren verfügen 21 der BondingMSO $(70 \%)^{115}$ über 1 bis 10 Engagierte und nur ein Fünftel von ihnen über Hauptamtliche (1, 2, 4 oder 9). Ein Drittel beklagt finanzielle Engpässe, wobei die betreffenden $10 \mathrm{MSO}$ zu gleichen Anteilen über weniger als 2, 10 oder 50 Tausend Euro pro Jahr verfügen. Mehrheitlich und fast paritätisch haben die Vereinigungen 11 bis 50,51 bis 100 oder mehr als 100 Mitglieder, in lediglich einer Vereinigung unter 10. Zudem zeigt sich, dass die Mitglieder unterschiedlichen Personengruppen $^{116}$ angehören, und zwar recht ausgewogen 2 bis 5 Personengruppen. In fast drei Viertel der Bonding-MSO stammen die Mitglieder aus dem gleichen Herkunftsland. Die dominierende Herkunftslandhomogenität fällt in 12 Fällen (40 \%) mit einer konfessionellen Homogenität zusammen. Diese doppelte Homogenität ist für 8 der insgesamt 12 unter den Bonding-Typen vertretenen religiösen MSO auszumachen, aber auch für 4 säkulare.

${ }^{114} 1947-1972=2$ MSO; $1973-1992=\underline{10}$ MSO; 1993-2004 = 7 MSO; 2005-2018 $=11 \mathrm{MSO}$ (Anmerkung: Kategorisierung der Gründungsjahre hier als vier Gruppen entlang zentraler gesetzlicher Maßnahmen als zeitliche Zäsuren in der deutschen Aus- bzw. Einwanderungspolitik).

115 Die Prozentangaben beziehen sich innerhalb dieses Analyseblocks jeweils auf die einem spezifischen Typus zugeordnete Anzahl von MSO in absoluten Zahlen (z. B. 30 Bonding-MSO $=100 \%)$.

116 Personengruppen bzw. Personenkreise: Berufstätige/Selbstständige, Schüler und Studierende, Hausfrauen/Hausmänner, Arbeitslose/Arbeitssuchende oder Rentner. 
Entsprechend der unter den Mitgliedern vorhandenen unterschiedlichen Personengruppen ist auch bei den Engagierten eine Durchmischung von Personenkreisen auszumachen. Allerdings sind die Engagierten bei einem Drittel der MSO - und damit häufiger als bei den Mitgliedern - einer Personengruppe zuzuordnen.

Im Gesamtbild lässt sich für die in den Vereinigungen involvierten Personen damit lediglich eine partielle Homogenität feststellen, die für die säkularen MSO insbesondere hinsichtlich der Herkunftsländer gilt. Darüber hinaus werden in nur einem Sechstel der Vereinigungen fremdenfeindliche Haltungen und in einem Fünftel der MSO eine negative öffentliche Meinung registriert, wovon in 4 Fällen (einem guten Zehntel) beides zugleich bejaht wird. Die Hälfte der Bonding-MSO ist mit der städtischen Einbindung zufrieden, ein Drittel ist dies nicht und ein Sechstel hat dazu keine Angabe gemacht.

Die Inblicknahme der organisationalen Charakteristika lässt insgesamt keine musterhaften, regelhaft in Kombination bestehenden Zusammenhänge mit den Bonding-Ausrichtungen erkennen. Finanzielle Mängel, eine längere oder kürzere Zeit des Bestehens der Organisationen oder wahrgenommene Diskriminierungen bzw. Negativ-Diskurse sind anhand der Tabellenübersicht (Anhang II im elektronischen Zusatzmaterial) augenscheinlich nicht als für bonding ursächlich auszumachen. Dies gilt auch für die Zuordnung zu religiösen Traditionen wie auch eine nur teilweise vorhandene Homogenität der Mitgliedereigenschaften. Der Befund zur doppelten Homogenität (40\%) in seiner Relevanz für BondingAusrichtungen ist im Weiteren im Vergleich mit anderen (Bridging-)Typen zu beurteilen.

Hinsichtlich der organisationalen Charakteristika der Bonding-Typen lassen sich auf der deskriptiven Ebene im Rahmen des qualitativ ausgerichteten Analyseschritts also keine eindeutigen bzw. einfachen Erklärungen ermitteln.

Dieses Urteil wird auch gestützt bei einer Betrachtung der $\mathbf{2 5}$ als Bridging II deklarierten Organisationen, d. h. der ,nicht-kooperierenden Aufgeschlossenen“ (Übersicht 7.1). Deren Organisationsmerkmale zeigen viele Ähnlichkeiten mit den Bonding-Typen: 44 Prozent der MSO geben finanzielle Mängel an; zudem herrscht in rund der Hälfte der Vereinigungen in Bezug auf das Herkunftsland und in 40 Prozent hinsichtlich der Konfession der Mitglieder eine Homogenität, die in 7 Vereinigungen $(28 \%$ ) - darunter 4 religiöse (von insgesamt 8 ) und 3 säkulare - zusammen auftreten.

In über drei Viertel dieser Bridging-MSO sind 1 bis 10 Engagierte vorzufinden, in einem Fünftel auch hauptamtliches Personal ( $3 \times$ eine Person, $2 \times 10$ Personen). Lediglich 2 MSO registrieren Fremdenfeindlichkeit und 3 Organisationen nehmen negative öffentliche Ansichten über die eigene Gruppe wahr. Über die Hälfte der MSO ist mit der städtischen Einbindung zufrieden. 
Deutlich wird, dass hinsichtlich der Zuordnungen von bridging oder bonding in Bezug auf die oben betrachteten Fälle und den dort vorzufindenden internen Verhältnissen grundsätzlich viele Ähnlichkeiten festzustellen sind. Insofern lassen sich keine Prototypen oder „One-fits-all“-Erklärungsmodelle identifizieren; Sozialkapital-Profile sind demnach nicht durch Organisationsmerkmale (prä-) determiniert oder eindeutig vorhersehbar. Dies erscheint nachvollziehbar: Wenn allgemein davon ausgegangen werden kann, dass eine gute Ressourcenausstattung eine nach außen gerichtete Handlungsfähigkeit begünstigt, könnte umgekehrt zutreffen, dass auch bei nachteiligeren Verhältnissen und geringeren Handlungsspielräumen, ggf. auf Basis gemeinsamer Wertegrundlagen, immaterielle bzw. personelle Ressourcen mobilisiert werden und vitale Kooperationen entstehen können, wenn dafür seitens der MSO Bedarfe und Realisierungsmöglichkeiten gesehen werden.

Obgleich sich das Erkennbare nicht ohne Weiteres auf einen gemeinsamen Nenner bringen lässt, kann eine tentative Schlussfolgerung dennoch lauten, dass Zusammenhänge und vorgefundene Verhältnisse begründende Faktoren nicht gänzlich zufällig oder kontingent sind. So zeigt ein Vergleich der eben fokussierten Bonding- und Bridging II-Typen mit den Bonding/Bridging-Typen - den „(hoch-)aktiven Brückenbauern“ - nämlich in Bezug auf die Verfügbarkeit von finanziellen Ressourcen sowie Engagierten eine deutlich geringere Ausstattung der beiden erstgenannten Typen. Die 17 den Bonding/Bridging-Typen subsumierten MSO sind in sehr offensichtlicher Weise deutlich mitglieder-, engagierten- und finanzstärker (vgl. Anhang II im elektronischen Zusatzmaterial). Über die Hälfte verfügen über hauptamtliche Mitarbeiter, darunter befinden sich drei Organisationen mit 5 bis 7 und eine mit 15 Hauptamtlichen. Wie auch bei den Bonding-Typen gehören die Mitglieder unterschiedlichen Personengruppen an, bei den Bonding/Bridging-MSO gilt dies jedoch auch in auffälliger Weise für die Engagierten. $11 \mathrm{MSO}$ dieses Typus (65\%) ordnen sich einer religiösen Tradition $\mathrm{zu}$; in 5 Fällen (29\%) ist die Koinzidenz einer Homogenität von Konfession und Herkunftsland der Mitglieder festzustellen. Zudem sind auch bei diesem Typus die Gründungsjahre der MSO über Dekaden sehr breit gestreut. Im Vergleich zu den Bonding-Typen zeigt sich anteilig geringfügig häufiger eine größere Zustimmung zu der Wahrnehmung von fremdenfeindlichen Positionierungen sowie negativen Meinungen. Mehr als die Hälfte der MSO ist mit der städtischen Einbindung zufrieden. Die ausgeprägtere Perzeption von Fremdenfeindlichkeit und negativen Sichtweisen lässt sich auf den religiösen Charakter der Vereinigungen zurückführen (7.1.7). Insgesamt lässt sich für die drei Aspekte (Fremdenfeindlichkeit; negative Meinung; städtische Einbindung) anhand der Tabelle ein differenziertes, nicht pauschalisiertes Antwortverhalten feststellen. 
Eine zusätzliche Inblicknahme der $\mathbf{3 0}$ dem Typus Bridging IV zugeordneten Vereinigungen, die keine Zielgruppen, aber Mitgliedschaftsbedingungen festlegen und die mit anderen Einrichtungen kooperieren, zeigt in der Summe ebenfalls disparate Formierungen und insbesondere hinsichtlich finanzieller und personeller Ressourcenstärke eine große Ähnlichkeit mit den Bonding/Bridging-Typen. Über die Hälfte dieser Bridging-MSO hat deutlich mehr als 100 Mitglieder. Ein Fünftel gibt Zahlen von 400, 800, 930, 940, 1.000 und 2.000 an. Allgemein befinden sich unter den Mitgliedern mit 4 bis 5 Personengruppen sehr vielfältige Gruppenzugehörigkeiten. In der Hälfte der Vereinigungen ist in Bezug auf das Herkunftsland und in 40 Prozent (12 MSO) hinsichtlich der Konfessionszugehörigkeit eine Einheitlichkeit festzustellen, wobei diese Homogenitäten in 6 religiösen Gemeinden $(20 \%)$ konvergieren. Insgesamt handelt es sich bei 13 MSO des Bridging IV-Typus (43\%) um religiöse Vereinigungen. In 40 Prozent der MSO gibt es hauptamtliches Personal ${ }^{117}$. Jeweils 8 MSO (27\%) nehmen Fremdenfeindlichkeit und negative öffentliche Meinungen über die eigene Gruppe wahr, wobei die Zustimmungen zu den beiden Phänomenen bei einem Sechstel der MSO koinzidieren. Über 50 Prozent der MSO dieses Typus sind mit der städtischen Einbindung zufrieden; 50 Prozent - und damit deutlich mehr als bei den Bonding/Bridging-Typen - beklagen finanzielle Mängel.

\section{$\checkmark$ Zwischenfazit}

Bedeutungsüberschätzung organisationsinterner Homogenität für bonding - Relevanz personeller Ressourcen für bridging

Die allgemein dominierende Homogenität von Herkunftsländern verweist auf die innerhalb der Zusammenschlüsse bestehenden Gemeinsamkeiten und Bindungen. Dass diese Einheitlichkeit in religiösen Vereinigungen häufiger mit einer konfessionellen Homogenität zusammenfällt, ist sinnfällig. Homogenität kommt hier insbesondere aufgrund familiärer Sozialisation, geteilter religiöser Überzeugungen und damit einhergehender (Selbst-)Selektionsprozesse zustande (II. 4.2.5; II. 4.4.2). Allerdings ist von einer solchen Koinzidenz nicht automatisch auszugehen und sie wird insbesondere in den religiösen Gemeinden wiederum flankiert von heterogenen sozioökonomischen Status der Mitglieder ${ }^{118}$. Dies ist mit großer Sicherheit auch mit dem Phänomen zu erklären, dass religiöse migrantische Gemeinden in der diasporischen Minderheitssituation Anlaufstelle für Angehörige ganzer Großfamilien und landsmannschaftlicher Netzwerke sind (II. 4.4.2). Im Gesamtbild lässt sich die

117 7-mal 1 Person; einmal 2 Personen; 4-mal 4-6 Personen.

118 Als Indiz fungiert die Mischung unterschiedlicher Mitgliedergruppen. 
Homogenitätsthese im Rahmen dieser analytischen Vorgehensweise nicht als für Bonding-Orientierungen ursächlich bestätigen. Dies ist allerdings vorbehaltlich der geringen Fallzahlen zu konstatieren und mit Verweis auf die Beobachtung, dass bei den Bonding-Typen (I - III) konfessionelle und herkunftsbezogene Homogenität in einem höheren Ausmaß koinzidieren.

Es scheint die Kombination von personellen Ressourcen (v. a. über 100 Mitglieder und viele Engagierte) mit finanzieller Stärke, die durchaus auch mit Engpässen einhergeht, in Verbindung mit einer - nicht spezifizierbaren - Mischung von homogenen und heterogenen Eigenschaften der Mitglieder zu sein, die die MSO befähigen, sowohl nach innen als auch nach außen beziehungsstark und vielfältig aktiv zu sein. Die Konstellationen der als (hoch-)aktiv bezeichneten Bonding/Bridgingsowie Bridging IV-Typen zeigen, dass die betreffenden MSO imstande sind, sowohl mit unterschiedlichen Abgrenzungsbedürfnissen einhergehende enge Binnengruppenbeziehungen als auch die Grenzen der eigenen Gemeinschaft überschreitende Verbindungen und Kontakte zu anderen (Kollektiv-)Akteuren zugleich zu pflegen.

Anhand der Analyse lässt sich schlussfolgern, dass die Verfügbarkeit personeller Ressourcen bedeutsamer ist als das Vorhandensein von genügend Geld. Allerdings ist herauszustellen, dass sich auch, wenn keine finanziellen Mängel, viele Mitglieder und auch (mehrere) Hauptamtliche vorhanden sind, Bonding-Performanzen ausprägen können (vgl. Typus I - III). Eine bestehende Verbandszugehörigkeit ist kein Indikator für spezifisch ausgerichtete kollektive Aktivitäten.

Ambivalentes und integratives Potenzial religiösen Engagements

Mit Blick auf die Relevanz der Zugehörigkeit der MSO zu religiösen Traditionen lässt sich vor diesem Hintergrund schlussfolgern, dass religiöses Engagement in Spannungsfeldern zu verorten ist und die positiven Effekte religiösen Sozialkapitals für gesellschaftliche Integrationsprozesse keinesfalls zu relativieren sind. Insbesondere in Anbetracht der Bridging IV- und Bonding/Bridging-Typen kann angenommen werden, dass die in Kombination mit einer religiösen Orientierung zutage tretenden organisationalen Eigenschaften sowohl nach innen bindende als auch nach außen brückenbauende Ausrichtungen bewirken. Zudem scheinen die Größe und Komplexität der Gruppen, die Pluralität der Mitglieder und womöglich auch die Vielfalt ihrer Ansprüche einer für ausschließliche Bonding-Performanz notwendige Überschau- und Kontrollierbarkeit der Binnenverhältnisse sowie einseitigen Gruppenorientierungen entgegenzustehen. Neben der Ressourcenlage können auch Außenweltbedingungen und ggf. vorhandene organisationsinterne und -externe Handlungserwartungen eine Rolle spielen, die im Rahmen dieser Erhebung jedoch nicht näher beleuchtet werden konnten. 
Die Analyseergebnisse zeigen, dass von einer rigorosen Einordnung von Religionen in entweder bridging oder bonding Abstand zu nehmen ist. Sowohl unter den Bonding- als auch Bridging-Typen befinden sich islamische, alevitische und unterschiedliche christliche (auch orthodoxe) Religionen. Damit werden die für unterschiedliche Religionen erfolgten disparaten theoretischen Bewertungen in Hinblick auf deren vertrauensgenerierenden und zivilgesellschaftlichen Funktionen (II. 4.2.4) durch die Lebenswirklichkeit ad absurdum geführt. Die Analyse legt vielmehr nahe, dass der Einfluss unterschiedlicher religiöser Dogmen auf das Handeln nicht überschätzt werden sollte und sich v. a. nicht theoretisch modellieren lässt. Ein solches Schubladendenken entbehrt auch wissenschaftlichen Erkenntnissen. Denn Religionen stellen keine monolithischen, statischen Blöcke dar, sondern können sich mit ihrer „Eigensinnigkeit“" verändern und auch eine Binnenheterogenität aufweisen. Als existenz- und kontextbezogene Systeme stehen sie mit ihren jeweiligen Umwelten kontinuierlich in Wechselwirkung und werden durch diese geformt. Bedingt durch geografische, kulturelle, soziale Kontexte sind Religionen Wandlungsprozessen unterworfen (vgl. Exc WWU 2018: 13-18).

\subsubsection{Erstellung von Summenindizes für statistische Cluster- und Regressionsanalysen}

Mit den bonding und bridging zugeordneten Variablen (Tab. 7.29, 7.2.1) sollen im Folgenden für statistische Evidenz weitere Rechnungen durchgeführt werden. Daher werden mit für die Organisationsziele und Aktivitäten selektierten Items Summenindizes erstellt. ${ }^{119}$ Die Indizes werden gebildet, indem jeweils eine bestimmte Anzahl von Variablen addiert wird. Die „gestaffelten“ Summenindex-Variablen bzw. mit ihnen erzeugten Skalen können damit unterschiedliche Minimal- und Maximalwerte annehmen. Je höher die Werte ausfallen, desto stärker ist auch die Bridging- bzw. Bonding-Performanz einzustufen. Mit

${ }^{119}$ Für die Bildung der Summenindizes wurden recodierte Variablen verwendet, sodass ein höherer Wert eine höhere Zustimmung darstellt: Für die Skalenwerte ,Trifft voll und ganz zu“ (Code 1), ,Trifft eher zu“ (2), ,Trifft eher nicht zu“ (3), ,Trifft überhaupt nicht zu“ (4) erfolgte eine inverse Recodierung $(4=1 ; 3=2 ; 2=3 ; 1=4)$.

Zum Umgang mit fehlenden Werten: Das generierte Gesamt-Scoring nimmt dann einen fehlenden Wert (-77) an, wenn eine Organisation auf nur einer der Subdimensionen einen fehlenden Wert aufweist.

Für die Summenindizes kommen folgende Fallzahlen (n) zustande:

$>$ Ziele-Bridging $<=225$ | $>$ Ziele-Bonding $<=215$ | $>$ Ziele-Bonding-Religion $<=214$ ।

$>$ Aktivitäten-Bridging $<=210$ | >Aktivitäten-Bridging-Religion $<=207$ । $>$ AktivitätenBonding $<=213$ | $>$ Aktivitäten-Bonding-Religion $<=208$. 
der Generierung der Summenindizes verknüpft ist die Intention, dass sie ohne und mit religiösen Subdimensionen gebildet, d. h. dass aufaddierte ,religiös neutrale“ Items in einer weiteren Skala jeweils um eine religiöse Subdimension ergänzt wurden. Konkret handelt es sich um folgende inhaltlich unterschiedlich aufgeladene Summenindizes bzw. Skalen (die religiösen Subdimensionen sind jeweils unterstrichen, die betreffenden Variablen jeweils mit dem Zusatz „Religion“ benannt worden):

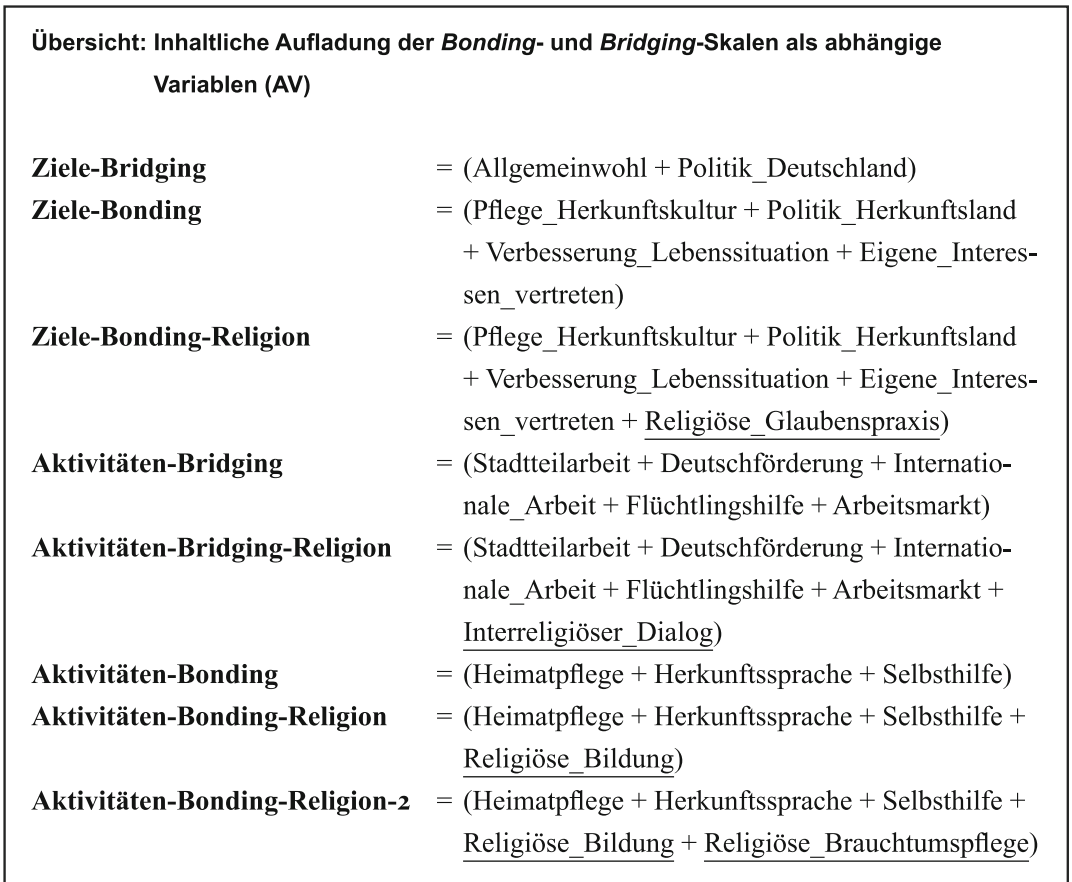

\subsubsection{Clusteranalysen}

Auf Basis von für die Aktivitäten und Organisationsziele generierten Skalen wurde zunächst eine statistische Clusteranalyse durchgeführt. Mit diesem explorativen Analyseverfahren wird mithilfe eines Algorithmus untersucht, ob in sich homogene Gruppen (Cluster) von MSO identifiziert werden können, die sich von anderen Gruppen möglichst stark absetzen. Dabei ist von Interesse, wie viele 
Cluster mit welchen Eigenschaften sich ergeben und wie sich die MSO auf die Cluster verteilen.

Anhand von vierstufigen Clusterungen, deren Prozedere und Resultate für den Nachvollzug im Folgenden detaillierter ausgeführt werden, zeigen sich RegelmäBigkeiten, die bereits bei der Veranschaulichung des Zusammenspiels von bonding und bridging zu Beginn dieses Kapitels sichtbar wurden (7.2.2): Religiöse und nicht-religiöse MSO gerieren sich hinsichtlich ihrer Sozialkapitalausprägungen sehr ähnlich, sobald man die religiösen Subdimensionen ausklammert. Denn bei der Clusterung mit den religiös neutralen Bridging- und Bonding-Skalen, die einerseits brückenbildende, andererseits binnenorientierte Merkmalsausprägungen der MSO umfassen, zeigen sich für die beiden Organisationskategorien jeweils sehr ähnliche Verteilungen auf die zwei generierten Cluster. Den religiösen Eigenschaften kommen hingegen eigenständige Effekte $\mathrm{zu}$, die mit anderen Bondingund v. a. auch Bridging-Ausprägungen kompatibel sind. Für die vier Konfessionen Islam, Alevitentum, Christentum und Judentum lassen sich in Hinblick auf die vier Clusterungen wiederum graduelle Unterschiede feststellen, die im Gesamtbild auf ein Kontinuum von bridging und bonding mit mehr oder weniger stark ausgeprägten Konvergenzen bzw. Divergenzen verweisen.

Vorgehensweise und einzelne Resultate der vier Clusterungen

Es erfolgte eine schrittweise Vorgehensweise: Auf Basis von jeweils vier Skalen wurden mit und ohne religiöse(n) Subdimensionen vier Clusterungen vorgenommen. Von der Einspeisung von weiteren Variablen, die spezifische Organisationsmerkmale beschreiben, wurde abgesehen, da damit das hohe Risiko einhergeht, dass sie sich stärker gegenüber den Bridging- und Bonding-Skalen durchsetzen und dann v. a. eine Einteilung nach diesen zusätzlichen Variablen als Ergebnis generiert wird, was unterbunden werden sollte. ${ }^{120}$

${ }^{120}$ Clusterung 1. und 3. Durchgang jeweils auf Basis von vier Skalen mit religiösen Subdimensionen: >Ziele-Bridging < I >Ziele-Bonding-Religion < | >Aktivitäten-Bridging-Religion< I >Aktivitäten-Bonding-Religion< - Clusterung 2. und 4. Durchgang jeweils auf Basis von vier Skalen ohne religiöse Subdimensionen: >Ziele-Bridging< I $>$ Ziele-Bonding< I $>$ Aktivitäten-Bridging $<1>$ Aktivitäten-Bonding $<$. 
In einem ersten Durchgang wurde zunächst hierarchisch-agglomerativ geclustert, wobei die Skalenniveaus als quasimetrisch betrachtet werden (Proximitätsmaß: Quadrierte Euklidische Distanz; Algorithmus: Ward-Verfahren bzw. „Minimum-Varianz-Methode“) ${ }^{121}$. Das Dendrogramm legte jeweils eine ZweiCluster-Lösung nahe: Cluster $\mathbf{A}_{\mathbf{1}}$ umfasst 115 Fälle, Cluster $\mathbf{B}_{\mathbf{1}} 68$ Organisationen. Zur Interpretation der Cluster-Charakteristika wurden deskriptive Statistiken verwendet. Die Mittelwertsunterschiede zwischen den Clustern zeigen an, dass Cluster $\mathbf{B}_{1}$ im ersten Durchlauf mit den religiösen Subdimensionen für alle Ziele und Aktivitäten mehr bridging und bonding ist als das Cluster $\mathbf{A}_{1}$. Während sich circa zwei Drittel der säkularen MSO auf das Cluster $\mathbf{A}_{1}$ verteilen, sind die religiösen MSO zu jeweils rund 50 Prozent paritätisch auf beide Cluster verteilt (Tab. 7.36).

Tab. 7.36 Clusterung 1 mit religiösen Skalen (Ward-Verfahren) (in absoluten und relativen Häufigkeiten)

\begin{tabular}{|c|c|c|c|}
\hline Cluster & Gesamt & Nicht-religiöse MSO & Religiöse MSO \\
\hline$A_{1}$ & $115[63 \%]$ & $83[67 \%]$ & 32 [53\%] \\
\hline $\begin{array}{l}\mathbf{B}_{1} \\
\text { (= mehr bridging/mehr bonding) }\end{array}$ & $68 \quad[37 \%]$ & $40[33 \%]$ & 28 [47\%] \\
\hline Gesamt & 183 & 123 & 60 \\
\hline
\end{tabular}

Ein zweiter Durchgang des hierarchisch-agglomerativen Verfahrens mit den religiös neutralen Skalen resultiert in zwei Clustern, wobei Cluster $\mathbf{A}_{2}$ hinsichtlich aller Skalen als mehr bridging und bonding zu charakterisieren ist. Im Cluster $\mathbf{A}_{2}$ finden sich nun zu jeweils zwei Dritteln sowohl religiöse als auch nicht-religiöse MSO (Tab. 7.37).

${ }^{121}$ Beim hierarchischen Agglomerieren (,Zusammenballen“) werden die Datenpunkte zuerst einzeln betrachtet und dann auf Basis eines Proximitäts- bzw. Distanzmaßes, das in Abhängigkeit des Skalenniveaus zu wählen ist und das die (Un-)Ähnlichkeit zwischen den zu clusternden Objekten ermittelt, schrittweise zu Clustern zusammengefasst. Mithilfe des WardVerfahrens (d. h. eines Cluster-Algorithmus, der innerhalb agglomerativer Clusterungen am leistungsstärksten ist und der am häufigsten angewendet wird) werden diejenigen zwei Cluster fusioniert, die die minimalste Varianzerhöhung im neuen Cluster erzeugen (vgl. Brosius 2018: 825-855).

Es ist darauf hinzuweisen, dass die Skalen mit mittleren und starken Effekten auf dem 0,01-Signifikanzniveau miteinander korrelieren. Dies widerspricht der Voraussetzung unkorrelierter Variablen, um das Ward-Verfahren als einen sehr guten Fusionierungsalgorithmus betrachten zu können (vgl. Backhaus et al. 2018: 470). 
Tab. 7.37 Clusterung 2 mit religiös neutralen Skalen (Ward-Verfahren) (in absoluten und relativen Häufigkeiten)

\begin{tabular}{|c|c|c|c|}
\hline Cluster & Gesamt & Nicht-religiöse MSO & Religiöse MSO \\
\hline $\begin{array}{l}\mathbf{A}_{2} \\
(=\text { mehr bridging } / \text { mehr bonding })\end{array}$ & $122[67 \%]$ & $82[67 \%]$ & 40 [67\%] \\
\hline $\mathrm{B}_{2}$ & $61[33 \%]$ & $41 \quad[33 \%]$ & $20[33 \%]$ \\
\hline Gesamt & 183 & 123 & 60 \\
\hline
\end{tabular}

Auch anhand der zweistufigen Ausführung der hierarchischen Clusterung mit jeweils vier Skalen bestätigt sich somit insgesamt, dass sich religiöse und nichtreligiöse MSO hinsichtlich ihrer Sozialkapitalausprägungen sehr ähnlich gerieren, sobald man die religiösen Subdimensionen ausklammert (vgl. 7.2.2).

Diese Ergebnisse lassen sich auch anhand des nicht-hierarchischen Verfahrens der Clusterzentrenanalyse bzw. einer K-Means-Clusterung (mit dem k-MeansAlgorithmus) bestätigen. Diese wird häufiger für eine Verbesserung einer auf Basis von hierarchischen Methoden erfolgten Gruppierung eingesetzt. Bei diesem werden die Cluster durch ihre Mittel- bzw. Schwerpunkte repräsentiert (Bortz 1999: 560 f.; Brosius 2018: 861 ff.) ${ }^{122}$. Auf Basis der mit der hierarchischen Clusterung zuvor ermittelten Zwei-Cluster-Lösung (Ward-Verfahren) wurde eine Anfangspartition mit zwei Clustern festgelegt. Die Clusterzentren der endgültigen Lösungen zeigen bei der Berücksichtigung der religiösen Subdimensionen ein Cluster $\mathbf{A}_{\mathbf{3}}$ mit 82 und ein Cluster $\mathbf{B}_{\mathbf{3}}$ mit 101 Fällen, das hinsichtlich der vier Skalen abermals sowohl als mehr bridging als auch mehr bonding zu charakterisieren ist. Hier finden sich circa zwei Drittel der religiösen und etwa die Hälfte der säkularen Organisationen (Tab. 7.38).

Tab. 7.38 Clusterung 3 mit religiösen Skalen (K-Means-Cluster) (in absoluten und relativen Häufigkeiten)

\begin{tabular}{|c|c|c|c|}
\hline Cluster & Gesamt & Nicht-religiöse MSO & Religiöse MSO \\
\hline$A_{3}$ & $82[45 \%]$ & $63[51 \%]$ & $19[32 \%]$ \\
\hline $\begin{array}{l}\mathbf{B}_{3} \\
\text { (= mehr bridging/mehr bonding) }\end{array}$ & $101[55 \%]$ & 60 [49\%] & 41 [68\%] \\
\hline Gesamt & 183 & 123 & 60 \\
\hline
\end{tabular}

${ }^{122}$ Bei der Clusterzentrenanalyse erfolgt eine Zuordnung jedes Objektes zu demjenigen Cluster, zu dessen Zentrum (d. h. dessen mittleren Werten) seine Distanz am geringsten ist. Bei diesem Verfahren kann auf die aufwendigen paarweisen Vergleiche im Rahmen hierarchischer Clusteranalyse verzichtet werden (vgl. Brosius 2018: 861 f.). 
Im Vergleich zu der allerersten Clusterung (Ward-Verfahren) mit den religiösen Skalen (Tab. 7.36) zeigt sich hier, dass sich die Verhältnisse für die religiösen und säkularen MSO quasi umkehren: Während sich die nicht-religiösen Vereinigungen paritätisch auf beide Cluster verteilen, sind im zweiten Cluster mehr religiöse zu finden. Die Aussage bleibt aber die gleiche: Säkulare MSO sind in Bezug auf die einbezogenen Skalen weniger bonding/bridging, religiöse hingegen mehr.

Eine Berechnung der Clusterzentren mit den religiös neutralen Skalen kommt im Vergleich zum vorigen Durchgang zu einem fast identischen Ergebnis von Clustern mit Blick auf Fallzuordnungen (+/-1) und Charakterisierungen, wobei sich die religiösen und säkularen MSO hier hinsichtlich ihrer Performanzen wiederum ähneln: Die religiösen verteilen sich jeweils ungefähr hälftig auf die beiden Cluster, während die säkularen mit 58 Prozent etwas häufiger im Cluster mit einer höheren Bridging/Bonding-Stärke vorzufinden sind (Tab. 7.39).

Tab. 7.39 Clusterung 4 mit religiös neutralen Skalen (K-Means-Cluster) (in absoluten und relativen Häufigkeiten)

\begin{tabular}{|c|c|c|c|}
\hline Cluster & Gesamt & Nicht-religiöse MSO & Religiöse MSO \\
\hline$A_{4}$ & $81[44 \%]$ & $52[42 \%]$ & $29[48 \%]$ \\
\hline $\begin{array}{l}\mathbf{B}_{4} \\
(=\text { mehr bridging/mehr bonding })\end{array}$ & $102[56 \%]$ & $71[58 \%]$ & $31[52 \%]$ \\
\hline Gesamt & 183 & 123 & 60 \\
\hline
\end{tabular}

Die Ergebnisse der Clusterungen, die ausschließlich auf Basis von Zielen und Aktivitäten der Organisationen erfolgten, sind methodisch und inhaltlich freilich nicht mit denjenigen der Typenbildungen vergleichbar, da bei diesen durch den Einbezug anderer Variablen eine differente, umfassendere und auch inhaltlichinterpretative Analyseperspektive auf die vorgefundenen Konfigurationen verfolgt wurde.

Auch anhand der statistischen Clusterungen zeigt sich im Gesamtbild, dass sich Unterschiede zwischen religiösen und säkularen MSO ausbalancieren, sobald die religiösen Subdimensionen eliminiert werden (vgl. auch 7.2.2). Mit Blick auf die vier Konfessionen Islam, Alevitentum, Christentum und Judentum zeigen die vier Clusterungen, dass sie sich auf beide Cluster verteilen (vgl. Tab. A 19 bis A 22 im elektronischen Zusatzmaterial). Wie auch bei der Analyse der gespiegelten Items zu Beginn des Kapitels ausführlich begutachtet (7.2.2), lassen sich sowohl zwischen den als auch innerhalb der Konfessionen graduelle Unterschiede feststellen. 
$\mathrm{Zu}$ konstatieren ist, dass Mehrwert und Erkenntnisgewinn der vorgenommenen Clusterungen zweifelsohne begrenzt sind, da zum einen eine positive Teststrategie verfolgt wird und zum anderen sowohl Bridging- als auch Bonding-Skalen in die Clusteranalyse hineingegeben wurden. Das Resultat von zwei Clustern - von einer auf die Items hoch sowie einer auf die Items niedrig ladenden Gruppe - ist demnach nicht überraschend; gleichwohl ermöglicht der auf theoretischen Vorentscheidungen basierende Ansatz, dass sich empirisch ein starkes Bonding zeigen könnte. Die Angleichung der religiösen und nicht-religiösen MSO bei Ausschluss der religiösen Subdimensionen spricht für die Robustheit der Unterscheidung des auf der Organisationsebene ansetzenden dichotomen Analysezugriffs ,säkular“ versus ,religiös“ und die Ergebnisse stützen damit insgesamt die Datenqualität (vgl. dazu auch $>$ Spotlight: Die religiöse Performanz der MSO).

\subsubsection{Multiple Regressionsanalysen}

Die bewusst offen formulierten Analysefragen (7.2), mit denen mögliche Erklärungszusammenhänge für Bonding- und Bridging-Performanzen fokussiert werden, wie etwa die finanzielle und personelle Ressourcenausstattung oder die Wahrnehmung von Fremdenfeindlichkeit ${ }^{123}$, lassen sich schärfer fassen, indem sie in ein Set von mit Regressionen statistisch überprüfbaren Hypothesen übersetzt werden. Diese Hypothesen lauten wie folgt:

${ }^{123}$ Vollständiger Wortlaut der Analysefragen (7.2; II. 4.2.5):

- Haben Binnenorientierung, fehlende Kontakte zu und fehlende Offenheit gegenüber der Umwelt mit Religion zu tun und lassen sich zwischen den Religionen Unterschiede aufdecken?

- Unter welchen Bedingungen bleiben die MSO unter sich?

- Lassen sich finanzielle Mängel, personelle Ressourcenausstattungen oder das Alter der Organisationen als Erklärungsfaktoren für auf die organisationale Binnenwelt gerichtete Performanzen identifizieren?

- Sind auszumachende Bonding-Tendenzen tatsächlich auf die theoretisch als ursächlich angenommene homogene Mitglieder- und Engagiertenstruktur zurückzuführen (Personengruppen, Konfessionszugehörigkeit, Herkunftsländer)?

- Inwieweit ist die Wahrnehmung von Fremdenfeindlichkeit, gesellschaftlichen Fehlplatzierungen und städtischer Nicht-Einbindung seitens der MSO zu registrieren und erklärt möglicherweise auf die eigene Gruppe konzentrierte Tätigkeiten? 
H8: Religiöse MSO produzieren mehr Bonding-Sozialkapital als nichtreligiöse.

H9: Je älter eine MSO ist, desto mehr Bonding-Sozialkapital produziert sie.

H10: Je homogener die Mitgliederstruktur einer MSO, desto mehr BondingSozialkapital produziert sie.

H11: Je ressourcenstärker eine MSO ist, desto mehr Bridging-Sozialkapital produziert sie.

H12: Je stärker eine MSO sie selbst betreffende Negativ-Diskurse wahrnimmt, desto mehr Bonding-Sozialkapital produziert sie.

H13: Je stärker eine MSO Fremdenfeindlichkeit und Diskriminierung wahrnimmt, desto mehr Bonding-Sozialkapital produziert sie.

H14: Je stärker sich eine MSO von der Stadt nicht ausreichend eingebunden fühlt, desto mehr Bonding-Sozialkapital produziert sie.

Mit der multiplen linearen Regression wird getestet, ob zwischen einer abhängigen und mehreren unabhängigen Variablen ein Zusammenhang besteht. Dabei unterliegt die lineare Regression diversen Modellprämissen (6.2). In den Modellen fungieren die Summenindex-Variablen bzw. die mit diesen erzeugten Skalen jeweils als abhängige Variablen (im Folgenden kurz: AV). Zusätzlich zu den bereits aufgeführten Skalen wurde eine weitere religiös akzentuierte Skala mit den zwei religiösen Subdimensionen „Religiöse Bildung/Erziehung“ und „Religiöse Brauchtumspflege" als AV in ein Modell aufgenommen $(\rightarrow>$ AktivitätenBonding-Religion-2 $\prec^{124}$ ).

${ }^{124}$ Aktivitäten-Bonding-Religion-2 = (Heimatpflege + Herkunftssprache + Selbsthilfe +

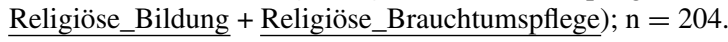




\section{Modellberechnungen I}

In die Modellberechnungen wurden als unabhängige Variablen (im Folgenden kurz: UV) in erster Linie (quasi-)metrische und zwei dichotome Variablen ${ }^{125}$ aufgenommen. ${ }^{126}$

Anhand der einzelnen Modelle lassen sich mit Blick auf die Hypothesen nachfolgende Ergebnisse berichten (s. Tab. 7.40) bzw. Schlussfolgerungen ziehen. Die Befunde sind in methodischer Hinsicht ebenfalls nicht mit denjenigen der Bonding- und Bridging-Typologisierung zu vergleichen. Zudem sind die Regressionsergebnisse aufgrund der geringen Fallzahlen und zum Teil niedrigerer Werte der korrigierten Bestimmtheitsmaße (korrigierte $\mathrm{R}^{2}$ ) nicht überzubewerten (Tab. 7.40). ${ }^{127}$

125 Anmerkungen zu den UV: Die dichotomen Variablen mit zwei Merkmalsausprägungen (Codierung 0/1 = nein/ja) betreffen die Zuordnung zu einer religiösen Tradition (nein/ja) sowie die Existenz von finanziellen Engpässen (nein/ja). Für die drei Variablen „Fremdenfeindlichkeit“, „Negative öffentliche Meinung“ und „Zufriedenheit mit städtischer Einbindung“ erfolgte eine inverse Recodierung $(4=1 ; 3=2 ; 2=3 ; 1=4)$, sodass ein höherer Wert eine höhere Zustimmung darstellt. Die fehlenden Werte der Variablen zur Anzahl von Engagierten und Ehrenamtlichen wurden dann „,0“ gesetzt, wenn die Existenz von Hauptamtlichen verneint wurde. Zur Bestimmung der Anzahl an vertretenen Konfessionen wurden die jeweiligen Angaben der MSO aufaddiert; Konfessionslosigkeit wurde mitgezählt, wenn sie neben anderen Konfessionen genannt wurde. Unvollständige Angaben (wenn z. B. lediglich über eine in der MSO mehrheitlich vertretene Konfession Auskunft gegeben wurde) wurden als fehlende Werte (-77) behandelt, da auf ihrer Basis keine konkrete Anzahl von Konfessionen zu ermitteln ist. Für die Festlegung der Anzahl der Herkunftsländer wurde bei einzelnen unspezifischen Angaben, die sich in neun Fällen auf einen Kontinent bezogen, der unter dem Item „Sonstiges“ genannt wurde (Afrika oder Subsahara-Afrika), mit dem Mittelwert der in der Stichprobe insgesamt genannten Herkunftsländer imputiert $(2,57 \approx 3 ; n=239)$. Die in drei Fällen unter „Sonstiges“ gemachte Angabe „International, keine Mehrheit von Herkunftsländern“"wurde jeweils als fehlender Wert (-77) deklariert.

${ }^{126}$ Methode: Einschluss (ENTER); Listenweiser Fallausschluss. Prüfung der Modellprämissen ist erfolgt (6.2).

Anmerkung zu den Berechnungen der Regressionsmodelle: Bei den Regressionen wurden Cook-Distanzen größer 0,5 entfernt (Fahrmeir 2009: 178; 6.2) und die Regressionen erneut durchgeführt bis keine weiteren einflussreichen Fälle mehr vorlagen.

${ }^{127}$ Für die finalen Modelle wurden jeweils korrigierte Bestimmtheitsmaße (korrigiertes $\mathrm{R}^{2}$ ) ermittelt (6.2), die die Varianzen der abhängigen Variablen durch die aufgestellten Modelle erklären. Die Prozentwerte fallen unterschiedlich aus. Beispiel: Für das erste Modell (AV: >Ziele-Bridging $<$ ) wurde ein korrigiertes $\mathrm{R}^{2}$ von 0,047 ermittelt, d. h. 4,7 \% der Varianz dieser $\mathrm{AV}$ werden durch das Modell erklärt. >Ziele-Bonding-Religion $<=\mathbf{1 9 , 4} \%$ I $>$ AktivitätenBridging $<=14,6 \%$ I >Aktivitäten-Bridging-Religion $<=9,1 \% \mathrm{I}>$ Aktivitäten-Bonding $<=$ 7,2 \% I >Aktivitäten-Bonding-Religion $<=26,2 \% \mathrm{I}>$ Aktivitäten-Bonding-Religion-2 $<=$ $\mathbf{4 1 , 4} \%$ (Tab. 7.40). 
Tab. 7.40 Modellberechnungen I: Multiple lineare Regressionsanalysen mit Bonding- und Bridging-Skalen als abhängige Variablen

\begin{tabular}{|c|c|c|c|c|c|c|c|c|}
\hline \multirow[b]{2}{*}{ Prädiktoren } & \multicolumn{8}{|c|}{ Abhängige Variablen } \\
\hline & $\begin{array}{l}\text { Ziele- } \\
\text { Bridging }\end{array}$ & $\begin{array}{l}\text { Ziele- } \\
\text { Bonding }\end{array}$ & $\begin{array}{c}\text { Ziele- } \\
\text { Bonding- } \\
\text { Religion* }^{*} \\
\text { (Glau- } \\
\text { bens- } \\
\text { praxis) }\end{array}$ & $\begin{array}{c}\text { Aktivi- } \\
\text { täten- } \\
\text { Bridging }\end{array}$ & $\begin{array}{l}\text { Aktivi- } \\
\text { täten- } \\
\text { Bridging- } \\
\text { Religion* } \\
\text { (Interrel. } \\
\text { Dialog) }\end{array}$ & $\begin{array}{l}\text { Aktivi- } \\
\text { täten- } \\
\text { Bonding }\end{array}$ & $\begin{array}{l}\text { Aktivi- } \\
\text { täten- } \\
\text { Bonding- } \\
\text { Religion* } \\
\text { (Rel. } \\
\text { Bildung) }\end{array}$ & $\begin{array}{c}\text { Aktivi- } \\
\text { täten- } \\
\text { Bonding- } \\
\text { Reli- } \\
\text { gion-2* } \\
\text { (Rel. } \\
\text { Bildung + } \\
\text { Brauch- } \\
\text { tum) }\end{array}$ \\
\hline Gründungsjahr & $\begin{array}{l}, 016^{*} \\
, 183 \\
\end{array}$ & & & & & & & \\
\hline $\begin{array}{l}\text { MSO: Zuord- } \\
\text { nung zu religiöser } \\
\text { Tradition (nein/ja) }\end{array}$ & & & $\begin{array}{l}2,364^{\star \star \star} \\
, 392\end{array}$ & & & & $\begin{array}{c}1,465^{\star \star} \\
, 235\end{array}$ & $\begin{array}{c}3,204^{\star * *} \\
, 422\end{array}$ \\
\hline $\begin{array}{l}\text { Finanzielle } \\
\text { Engpässe } \\
\text { (nein/ja) }\end{array}$ & & & & & & & $\begin{array}{l}, 937^{*} \\
, 169\end{array}$ & $\begin{array}{c}1,167^{*} \\
, 161\end{array}$ \\
\hline $\begin{array}{l}\text { Mitglieder Religi- } \\
\text { onszugehörigkeit } \\
\text { (Anzahl Gruppen) }\end{array}$ & & & & & & & & $\begin{array}{l}-, 353^{*} \\
-, 171\end{array}$ \\
\hline \multicolumn{9}{|l|}{$\begin{array}{l}\text { Mitglieder } \\
\text { Herkunftsländer } \\
\text { (Anzahl) }\end{array}$} \\
\hline \multicolumn{9}{|l|}{$\begin{array}{l}\text { Engagierte } \\
\text { (Anzahl) }\end{array}$} \\
\hline \multicolumn{9}{|l|}{$\begin{array}{l}\text { Hauptamtliche } \\
\text { (Anzahl) }\end{array}$} \\
\hline $\begin{array}{l}\text { Zufriedenheit } \\
\text { Einbindung in Stadt }\end{array}$ & & & & & & & $\begin{array}{l}, 593^{*} \\
, 197\end{array}$ & $\begin{array}{l}, 825^{\star *} \\
, 210\end{array}$ \\
\hline $\begin{array}{l}\text { Fremdenfeindlich- } \\
\text { keit/Diskriminierung }\end{array}$ & & & & & & $\begin{array}{l}, 562^{*} \\
, 227\end{array}$ & $\begin{array}{l}, 774^{* *} \\
, 268\end{array}$ & $\begin{array}{l}, 862^{* *} \\
, 230\end{array}$ \\
\hline \multicolumn{9}{|l|}{$\begin{array}{l}\text { Negative öffent- } \\
\text { liche Meinung }\end{array}$} \\
\hline Korrigiertes $\mathrm{R}^{2}$ &, 047 &,- 002 & , 194 & , 146 & ,091 & ,072 & ,262 & ,414 \\
\hline Fallzahlen & 156 & 150 & 149 & 147 & 145 & 152 & 147 & 145 \\
\hline
\end{tabular}

Multiple lineare Regressionen: Bericht von nicht standardisierten (obere Angabe) und standardisierten Regressionskoeffizienten nach Pearson $(\beta)$ (untere Angabe; für die Vergleichbarkeit der UV innerhalb eines Modells werden die standardisierten Koeffizienten angegeben, bei denen die Effekte unterschiedlicher Skalenniveaus herausgerechnet wurden). Signifikanz: $* * * \mathrm{p}<, 001 ; * * \mathrm{p}<, 01 ;{ }^{*} \mathrm{p}<, 05$; Methode: Einschluss (ENTER); Listenweiser Fallausschluss. * Die fett gesetzten AV mit dem Bezeichnungszusatz „Religion“ enthalten jeweils eine religiöse Subdimensionen (die in Klammern hinter der AV-Bezeichnung vermerkt ist). Eine Ausnahme ist die AV >Aktivitäten-Bonding-Religion-2 $<$, die zwei religiöse Subdimensionen umfasst (,Religiöse Bildung/Erziehung“ und „Religiöse Brauchtumspflege“). 
- Hypothese 8 bestätigt sich lediglich in Bezug auf die religiös aufgeladenen AV: Kollektive religiöse Bindungen haben in drei Modellen einen signifikanten Effekt und wirken jeweils erhöhend auf religiöses Bonding-Kapital. Im Sinne einer, selbsterfüllenden Prophezeiung ' ist dies nicht überraschend. Nimmt man darüber hinaus die Bridging-AV in den Blick, zeigt sich in Bezug auf die Zuordnung zu einer religiösen Tradition hingegen kein einziger signifikanter Effekt, und zwar auch nicht bei der den interreligiösen Dialog umfassenden Bridging-AV (>Aktivitäten-Bridging-Religion $\prec$ ). Dies unterstreicht, dass kollektive religiöse Bindungen religiöses Bonding-Kapital stimulieren.

- Hypothese 9 kann nicht bestätigt werden: Es zeigt sich kein einziger signifikanter Zusammenhang zwischen dem Organisationsalter und Bonding-Kapital. Allerdings hat das Gründungsjahr im ersten Modell (`Ziele-Bridging) einen signifikant positiven Einfluss und wirkt dabei erhöhend auf das Bridging-Kapital: Jüngere Organisationen verfolgen häufiger die Bridging-Ziele „Etwas für das allgemeine Wohl in Deutschland tun“ sowie „Parteipolitische Positionen in Deutschland mitgestalten/beeinflussen“. Dies ist ein Indiz dafür, dass der Zeitpunkt der Gründung einer MSO offensichtlich einen Einfluss auf kollektive Zielorientierungen hat.

- Hypothese 10 lässt sich wiederum lediglich in Bezug auf konfessionelle Homogenität im Zusammenhang mit der zweifach religiös konnotierten Bonding-AV $>$ Aktivitäten-Bonding-Religion-2< bestätigen. In Hinblick auf herkunftslandbezogene Binnenhomogenität zeigt sich hingegen kein einziges signifikantes Ergebnis.

Der statistisch signifikante negative Effekt bei der Bonding-AV, die die zwei Aktivitäten „Religiöse Bildung“ und „Religiöse Brauchtumspflege“ umfasst ( A Aktivitäten-Bonding-Religion-2 «), lässt sich dahingehend interpretieren, dass die Mitgliederstruktur derjenigen MSO, die sich mit ihren Aktivitäten stark auf die eigene religiöse Gruppe konzentrieren anstatt eine Außenorientierung zu verfolgen, religiös homogener ausfällt.

Die Tatsache, dass sich hinsichtlich der Anzahl der Herkunftsländer gar keine signifikanten Ergebnisse zeigen, ist angesichts der theoretisch als für BondingTendenzen ursächlich betrachteten homogenen Mitgliederstrukturen aufschlussreich. So wären für die Herkunftsländer etwa signifikante negative Effekte bei $\mathrm{AV}$ zu erwarten, die potenziell stark an einem Herkunftsland orientierte Aktivitäten umfassen (z. B. >Aktivitäten-Bonding ‘); positive Effekte wären hingegen bei $\mathrm{AV}$ zu erwarten, die herkunftslandübergreifende Aktivitäten umfassen, wie die 
AV >Aktivitäten-Bridging ${ }^{128}$. Hinsichtlich der Ergebnisse ist jedoch in Erwägung zu ziehen, dass die AV als Summenindizes wiederum Ziele und Aktivitäten umfassen, bei denen sich gleich- und verschiedenartige Zusammensetzungen der Mitglieder nicht zwangsläufig als potente Parameter durchschlagen (müssen).

- Hypothese 11 lässt sich als solche nicht bestätigen. Dass sich in Bezug auf die Gesamtzahl von Engagierten sowie Hauptamtlichen keinerlei Signifikanzen zeigen, überrascht, da davon ausgegangen werden könnte, dass ihre Verfügbarkeit eine Voraussetzung für eine Vielfalt von Tätigkeiten darstellt, wie sie die AV teilweise umfassen. Insbesondere die Anzahl von Hauptamtlichen könnte als Ermöglichungsbedingung von komplexeren Tätigkeiten, die spezifische Anforderungen mit sich bringen und Kompetenzen voraussetzen (wie sie v. a. die AV $>$ Aktivitäten-Bridging < einschließt), betrachtet werden.

In Hinblick auf die Engagierten ist zu beachten, dass auch für die Ausführung von Bonding-Aktivitäten personelle Ressourcen und die Verfügbarkeit von Engagierten vorauszusetzen ist, weshalb sich in Bezug auf für bridging und bonding angenommene Ressourcendiskrepanzen relativieren könnten. Insofern lassen sich - wie bei der Betrachtung der personellen Ressourcenausstattung einzelner Sozialkapital-Typen in diesem Kapitel (7.2.5.1) - auch hier keine eindeutigen Erklärungsmuster finden; Sozialkapital-Profile und Handlungsfähigkeit der MSO sind demnach nicht durch personelle Ressourcenstärke (prä-)determiniert oder eindeutig vorhersagbar.

Die Registrierung von finanziellen Engpässen wirkt sich in zwei Modellen aus: Die signifikant positiven Effekte auf das Bonding-Kapital bei den zwei jeweils religiöse Aktivitäten umfassenden Bonding-AV (>Aktivitäten-BondingReligion <; >Aktivitäten-Bonding-Religion-2<) lässt sich in zweierlei Richtungen mit entgegengesetzten „kausalen“Zusammenhängen deuten. Zum einen könnten sich MSO mit begrenzten finanziellen Ressourcen hinsichtlich ihrer Aktivitäten eher auf die eigene religiöse Gruppe konzentrieren anstatt eine Außenorientierung zu verfolgen. Zum anderen könnten stark religiös bonding agierende MSO, die weniger öffentliche Fördermittel empfangen (7.1.2.2), in Anbetracht einer großen Angebotsvielfalt und Bedarfsdeckung der in den Vereinigungen involvierten Personen (7.1.4) eher finanzielle Engpässe konstatieren.

- Weiterhin kann Hypothese 12 nicht bestätigt werden. Die Ergebnisse bezüglich der Wahrnehmung negativer öffentlicher Meinungen sind in keinem der Modelle signifikant.

$128>$ Aktivitäten-Bridging $<=($ Stadtteilarbeit + Deutschförderung + Internationale_Arbeit + Flüchtlingshilfe + Arbeitsmarkt). 
- Hypothese 13 kann hingegen bestätigt werden. Der Einfluss der Fremdenfeindlichkeit auf Bonding-Aktivitäten ist in drei Modellen signifikant positiv. Bezüglich der Wirkungen der UV, die sich auf die Wahrnehmung von Fremdenfeindlichkeit bezieht, ist hier auf die religiöse Aufladung der beiden AV zu achten $^{129}$. Im Rahmen der deskriptiven Auswertung (7.1.7) wurde gezeigt, dass die religiösen MSO in einem signifikanten Ausmaß mit mehr Fremdenfeindlichkeit als die säkularen konfrontiert sind. Die signifikanten Ergebnisse der Regressionen sind somit mit dem religiösen Charakter der Vereinigungen zu erklären. Jedoch zeigt sich auch bei der religiös neutralen Bonding-AV (>AktivitätenBonding<) ein positiver Effekt wahrgenommener Fremdenfeindlichkeit. Dies bedeutet, dass auch MSO, die ausschließlich nicht-religiösen Aktivitäten nachgehen, von Fremdenfeindlichkeit betroffen sind und könnte darauf verweisen, dass abwertendes, fremdenfeindliches Verhalten und Gedankengut - unabhängig von religiösen Ausrichtungen - tatsächlich die Konzentration auf die Herkunftskultur und die eigene Gruppe forciert. Unabhängig von dieser Deutungsmöglichkeit ist Fremdenfeindlichkeit auf jeden Fall als eine Begleiterscheinung der Aktivitäten der MSO einzustufen.

- Hypothese 14 kann wiederum nicht bestätigt werden. Hinsichtlich der Einbindung in die Stadt lässt sich der vermutete negative Zusammenhang nicht belegen, sondern - diametral entgegengesetzt - ein positiver: In zwei Modellen zeigen sich signifikant positive Effekte der Einbindung in die Stadt auf religiöses Bonding-Kapital. Diese treten jeweils zusammen mit den positiven Effekten von Fremdenfeindlichkeit auf. Die positiven Effekte der Zufriedenheit mit der städtischen Einbindung können dahingehend interpretiert werden, dass die Ausübung religiöser Aktivitäten und eine positiv wahrgenommene städtische Einbindung gut miteinander kompatibel sind. Die städtische Verwaltung, Politik und andere Akteure akzeptieren eine selbstbewusste religiöse Aktivitätsausübung und können damit verstärkende Wirkungen auf die religiöse Performanz haben, die auf städtischen Rückhalt bauen, aber angesichts xenophober, diskriminierender Haltungen auch als Rückzugsort fungieren kann. Die signifikanten Ergebnisse unterstreichen zugleich, dass die UV nicht allein als für die religiös konnotierten Bonding-Aktivitäten ursächlich betrachtet werden können, sondern dass diese Merkmalsausprägungen die Aktivitäten im signifikanten Ausmaß flankieren und damit auf ihre Vereinbarkeit mit der AV verweisen. In Anlehnung an die Studie von Miriam Schader (2017) (I. 2.2) lässt sich dies dahingehend interpretieren, dass die MSO die Religion in zweifacher Hinsicht nutzen können: einerseits

$129>$ Aktivitäten-Bonding-Religion $<$; Aktivitäten-Bonding-Religion-2く. 
als ein geteiltes, nach innen einheitsstiftendes Identitätsmerkmal und andererseits als in der (Stadt-)Gesellschaft legitimierter und Legitimität verschaffender Faktor (Schader 2017: 220).

\section{Modellberechnungen II}

Da insbesondere der Einfluss des Islam von Interesse ist, wurden die multiplen Regressionen in einem zweiten Schritt mit den religiösen Traditionen als Dummies getrennt nach ,Islam“ und ,Sonstigen Religionen“ (= Christentum, Alevitentum, Judentum, Ezidentum, Hinduismus) als UV durchgeführt. Als Referenzkategorie fungieren jeweils die nicht-religiösen MSO. Ansonsten wurden dieselben Variablen in die Modelle eingespeist und es wurde genauso vorgegangen wie bei den ersten Berechnungen. Im Folgenden werden lediglich Auffälligkeiten berichtet und Interpretationen vorgenommen, die mit den ersten Modellberechnungen nicht in Erscheinung getreten bzw. relevant geworden sind (Tab. 7.41).

Interessant sind die Ergebnisse für die religiösen Traditionen ,Islam‘ und ,Sonstige Religionen'. Im Vergleich mit jeweils nicht-religiösen Vereinigungen zeigen sich jeweils zwei positive Effekte für die nicht-islamischen Religionen bei den beiden AV >Ziele-Bonding ${ }^{130}$ sowie $>$ Ziele-Bonding-Religion $<{ }^{131}$. Bei der sich auf die Aktivitäten beziehenden AV >Aktivitäten-Bonding-Religion< ergibt sich hingegen lediglich für den Islam ein positiver Effekt; ein solcher ist für die AV >AktivitätenBonding-Religion-2 «allerdings wiederum für ,Islam ‘ und ,Sonstige Religionen ‘ zu ermitteln.

Wie lassen sich diese Ergebnisse deuten? Zum einen weisen sie darauf hin, dass die Aktivitäten der zweifach religiös konnotierten Bonding-AV ( $>$ AktivitätenBonding-Religion-2 $\iota$ ) sowohl für den Islam als auch für die anderen Religionen relevant sind. Zum anderen indizieren die in den genannten AV enthaltenen Bonding-Ziele, worauf das kollektive Handeln ausgerichtet sein soll(te). Da sie für die nicht-islamischen Religionen gewichtiger sind, wird die öffentliche Debatte um den problematischen Islam, wie sie zu Beginn dieses Auswertungskapitels aufgegriffen wurde (7.2), konterkariert. Denn Ziele sind als das zu verstehen, worauf das kollektive Wollen und Handeln bewusst ausgerichtet ist, um spezifische Interessen und Pläne umzusetzen. Die Ergebnisse der Regressionen implizieren somit eine größere Bedeutsamkeit eines ,eigenkulturellen Rückzugsort[s]“ (Baumann

\footnotetext{
$130>$ Ziele-Bonding $<=$ (Pflege_Herkunftskultur + Politik_Herkunftsland + Verbesserung_Lebenssituation + Eigene_Interessen_vertreten).

131 >Ziele-Bonding-Religion $<=$ (Pflege_Herkunftskultur + Politik_Herkunftsland + Verbesserung_Lebenssituation + Eigene_Interessen_vertreten + Religiöse_Glaubenspraxis).
} 
2004: 27) und der Stärkung der eigenen Gemeinschaft im Binnenraum der nichtislamischen MSO, was mit Grenzziehungen nach außen einhergeht. Der Islam bzw. die Muslime stehen hingegen stärker im öffentlichen Scheinwerferlicht und übernehmen in religions- und integrationspolitischen Fragen die Rolle von Ansprechund Kooperationspartnern für politische Akteure. Für die Muslime dürften die betreffenden Bonding-Ziele daher weniger relevant sein, was wiederum nicht bedeutet, dass sie hinsichtlich ihrer Aktivitäten nicht religiös bonding agieren. In den Ergebnissen der Regressionen spiegeln sich insofern erneut die mehrzweckorientierten Performanzen islamischer Organisationen wider und sie verdeutlichen, dass es nicht gerechtfertigt ist, islamfokussierte öffentliche Diskurse auf die (polarisierte) Sozialkapital-Debatte zu projizieren, sondern dass differenzierende und versachlichende Blickwinkel vonnöten sind.

Insgesamt zeigen die Ergebnisse der multiplen Regressionsanalysen ein differenzierteres Bild, das in Hinblick auf nicht vorhandene bzw. nicht durchgängig signifikante Effekte in Bezug auf die jeweiligen Bonding- und Bridging-AV keine pauschalen Erklärungen bietet. Die signifikanten Ergebnisse zeigen statistische Zusammenhänge zwischen Merkmalsausprägungen der UV und AV, jedoch keine Kausalitäten. Die Schlussfolgerungen sind damit tentative, denn über die Wirkrichtungen der signifikanten Effekte kann keine eindeutige Aussage getroffen und ihre Bedeutung für die Lebenswirklichkeit der betroffenen MSO kann entsprechend lediglich gemutmaßt werden. Zweifelsohne wäre es sinnvoll und erkenntniserweiternd, die Ergebnisse anhand höherer Fallzahlen zu überprüfen. 
Tab. 7.41 Modellberechnungen II: Multiple lineare Regressionsanalysen mit Bonding- und Bridging-Skalen als abhängige Variablen

\begin{tabular}{|c|c|c|c|c|c|c|c|c|}
\hline \multirow[b]{2}{*}{ Prädiktoren } & \multicolumn{8}{|c|}{ Abhängige Variablen } \\
\hline & $\begin{array}{l}\text { Ziele- } \\
\text { Bridging }\end{array}$ & $\begin{array}{l}\text { Ziele-Bon- } \\
\text { ding }\end{array}$ & $\begin{array}{l}\text { Ziele-Bon- } \\
\text { ding- } \\
\text { Religion* } \\
\text { (Glau- } \\
\text { bens- } \\
\text { praxis) }\end{array}$ & $\begin{array}{l}\text { Aktivi- } \\
\text { täten- } \\
\text { Bridging }\end{array}$ & $\begin{array}{l}\text { Aktivi- } \\
\text { täten- } \\
\text { Bridging- } \\
\text { Religion* } \\
\text { (Interrel. } \\
\text { Dialog) }\end{array}$ & $\begin{array}{l}\text { Aktivitä- } \\
\text { ten-Bon- } \\
\text { ding }\end{array}$ & $\begin{array}{l}\text { Aktivitä- } \\
\text { ten-Bon- } \\
\text { ding- } \\
\text { Religion* } \\
\text { (Rel. } \\
\text { Bildung) }\end{array}$ & $\begin{array}{c}\text { Aktivitä- } \\
\text { ten-Bon- } \\
\text { ding- } \\
\text { Reli- } \\
\text { gion-2* } \\
\text { (Rel. } \\
\text { Bildung + } \\
\text { Brauch- } \\
\text { tum) }\end{array}$ \\
\hline Gründungsjahr & $\begin{array}{l}, 015^{*} \\
, 178\end{array}$ & & & & & & & \\
\hline $\begin{array}{l}\text { MSO: Zuordnung } \\
\text { zu Islam (Referenz- } \\
\text { kategorie: säkulare } \\
\text { MSO) }\end{array}$ & & & & & & & $\begin{array}{c}1,727^{\star *} \\
, 246\end{array}$ & $\begin{array}{c}3,509^{\star * *} \\
, 379\end{array}$ \\
\hline $\begin{array}{l}\text { MSO: Zuordnung } \\
\text { zu anderen Reli- } \\
\text { gionen (Referenz- } \\
\text { kategorie: säkulare } \\
\text { MSO) }\end{array}$ & & $\begin{array}{c}1,403^{*} \\
, 212\end{array}$ & $\begin{array}{c}3,677^{\star \star \star} \\
, 458\end{array}$ & & & & & $\begin{array}{l}2,889^{\star * *} \\
, 293\end{array}$ \\
\hline $\begin{array}{l}\text { Finanzielle } \\
\text { Engpässe } \\
\text { (nein/ja) }\end{array}$ & & & & & & & $\begin{array}{l}, 884^{*} \\
, 160\end{array}$ & $\begin{array}{r}1,108^{*} \\
, 153\end{array}$ \\
\hline $\begin{array}{l}\text { Mitglieder Religi- } \\
\text { onszugehörigkeit } \\
\text { (Anzahl Gruppen) }\end{array}$ & & & & & & & & $\begin{array}{l}-, 332^{*} \\
-, 161\end{array}$ \\
\hline \multicolumn{9}{|l|}{$\begin{array}{l}\text { Mitglieder } \\
\text { Herkunftsländer } \\
\text { (Anzahl) } \\
\end{array}$} \\
\hline \multicolumn{9}{|l|}{$\begin{array}{l}\text { Engagierte } \\
\text { (Anzahl) }\end{array}$} \\
\hline \multicolumn{9}{|l|}{$\begin{array}{l}\text { Hauptamtliche } \\
\text { (Anzahl) }\end{array}$} \\
\hline $\begin{array}{l}\text { Zufriedenheit } \\
\text { Einbindung in Stadt }\end{array}$ & & & & & & & $\begin{array}{l}608^{\star \star} \\
, 201 \\
\end{array}$ & $\begin{array}{l}, 841^{* *} \\
, 214\end{array}$ \\
\hline $\begin{array}{l}\text { Fremdenfeindlich- } \\
\text { keit/Diskriminierung }\end{array}$ & & & & & & $\begin{array}{l}, 514^{*} \\
, 208\end{array}$ & $\begin{array}{l}, 726^{* *} \\
, 252\end{array}$ & $\begin{array}{l}, 807^{* *} \\
, 216\end{array}$ \\
\hline \multicolumn{9}{|l|}{$\begin{array}{l}\text { Negative öffentli- } \\
\text { che Meinung }\end{array}$} \\
\hline Korrigiertes $\mathrm{R}^{2}$ & ,046 &, 051 & ,247 & , 140 & ,085 & ,069 & ,259 & ,412 \\
\hline Fallzahlen & 156 & 150 & 149 & 147 & 145 & 152 & 147 & 145 \\
\hline
\end{tabular}

Multiple lineare Regressionen: Bericht von nicht standardisierten (obere Angabe) und standardisierten Regressionskoeffizienten nach Pearson $(\beta)$ (untere Angabe; für die Vergleichbarkeit der UV innerhalb eines Modells werden die standardisierten Koeffizienten angegeben, bei denen die Effekte unterschiedlicher Skalenniveaus herausgerechnet wurden). Signifikanz: $* * * \mathrm{p}<, 001 ; * * \mathrm{p}<, 01 ;{ }^{*} \mathrm{p}<, 05$; Methode: Einschluss (ENTER); Listenweiser Fallausschluss. * Die fett gesetzten AV mit dem Bezeichnungszusatz „Religion“ enthalten jeweils eine religiöse Subdimensionen (die in Klammern hinter der AV-Bezeichnung vermerkt ist). Eine Ausnahme ist die AV >Aktivitäten-Bonding-Religion-2 «, die zwei religiöse Subdimensionen umfasst (,Religiöse Bildung/Erziehung“ und „Religiöse Brauchtumspflege“). 


\subsubsection{Fazit}

\section{Bonding und bridging sind keine antagonistischen Ausschließungskategorien}

Im Gesamtbild bestätigt sich die Putnamsche Annahme, dass viele Gruppen bzw. Vereinigungen entlang unterschiedlicher (sozialer) Dimensionen Bonding- und Bridging-Performanzen zugleich an den Tag legen und dass die beiden Ausprägungsarten somit keine Entweder/Oder-Kategorien darstellen, in die soziale Gebilde eindeutig unterteilt werden können. Vielmehr handelt es sich um jeweils mehr oder weniger vorhandene Ausmaße der zwei Sozialkapitalformen (Putnam 2000: 23). Insofern bestätigt die Studie empirisch, dass der Grundsatzstreit über herkunftsidentitäts- oder integrationsfördernde Funktionen der MSO „durch differenziertere Perspektiven zu ersetzen“ ist, da MSO ,in der Regel beide Aufgaben gleichzeitig" erfüllen (Pries 2010a: 25; I. 2.2).

Die Analysen haben gezeigt, dass es sich um ein variierendes Zusammenspiel handelt. Mit der vorliegenden Studie wird die Bonding/Bridging-Debatte nicht grundsätzlich ad absurdum geführt, aber sie fördert Offenheit und eine hohe Aktivität der häufig unter Bonding-Verdacht stehenden religiösen MSO zutage. Die Ergebnisse sind vergleichbar mit denjenigen einer jüngeren Studie zur Bedeutung von nicht-migrantischen christlichen Kirchengemeinden in der Zivilgesellschaft (Ohlendorf/Rebenstorf 2019), aufgrund derer den Gemeinden eine ,überraschende" Offenheit gegenüber ihrer Umwelt attestiert wird; mit ihren ehrenamtlichen und gemeindlichen Strukturen seien sie besonders geeignet, zentrale Funktionen für das Gemeinwesen wahrzunehmen und die Sozialraumentwicklung voranzutreiben. Jedoch sei stets die Einflussnahme von disparaten lokalen Rahmenbedingungen zu bedenken.

Darüber hinaus bestätigen die hiesigen Befunde die Forschungsergebnisse des KONID-Survey 2019 ${ }^{132}$, die zeigen, dass Religion, ,auf der strukturellen Ebene der Organisation von zivilgesellschaftlichem Engagement [wirkt] [...] und damit zum brückenbildenden sozialen Kapital, d. h. zu den Ressourcen des gesellschaftlichen Zusammenhalts bei[trägt]“" (Liedhegener et al. 2019: 45). Anhand der Analysen verbieten sich pauschale Urteile zu spezifischen Wirkrichtungen einzelner religiöser Traditionen. Die empirischen Befunde dieser Studie, die über vergleichende, kleinschrittige Analysen ermittelt wurden, sind als Anhaltspunkt für ähnliche zivilgesellschaftliche Performanzen von religiösen und säkularen MSO zu betrachten und lagen als solche bislang nicht vor.

132 „,KONID“ = „,Konfigurationen individueller und kollektiver religiöser Identitäten und ihre zivilgesellschaftlichen Potentiale“. 


\section{Mehrfach-kulturelle Verortungen und Bezugssysteme}

Empirisch sichtbar werden anhand vorliegender Studie die Beibehaltung der Herkunftskultur und zugleich die Überwindung eines ausschließlichen Herkunfts(land)bezugs durch ein auch über die Stadtgesellschaft hinausgehendes breites Aktivitäts- und Kooperationsspektrum. Damit entsprechen die Ergebnisse dem für islamische Verbände im Rahmen quantitativer Untersuchungen festgestellten Befund, dass die Aktivitäten sowohl eine Hinwendung zur Aufnahmegesellschaft als auch binnenorientierte herkunftskulturelle Orientierungen zugleich widerspiegeln und in der Summe damit keine Integrationsresistenz erkennen lassen (vgl. Halm et al. 2012a: 5-8, 116-119; Sauer/Halm 2013: 413-415; Spielhaus/Mühe 2018: 44, $46,50 \mathrm{ff}$.).

Mit Blick auf Orientierungen am Herkunftsland und Deutschland zeigt sich kein ,Entweder-Oder', sondern ein ,Sowohl-als-Auch'. Die Pflege eines Abstammungserbes geht mit einer Öffnung nach außen und gesellschaftspolitischen Interessen und Aktivitäten einher: ,Herkunftsheimat“-Vereine können zugleich auch „Integrationsvereine“ sein, Herkunfts- und Deutschlandbezüge treten gleichzeitig auf und spiegeln damit bi- oder mehrfach-kulturelle Orientierungen. Mit „Herkunftsheimat“ ist ein außerhalb von Deutschland existierendes kulturelles Bezugs- und Orientierungssystem gemeint, das - auch ohne eine direkte räumliche Verbindung - aufgrund familiärer Prägungen oder historischer Verwurzelungen für die Individuen bedeutsam und identitätsstiftend ist (Uslucan 2017). Dies erscheint nachvollziehbar, wenn das Herkunftsland der (Groß-)Eltern bei einer Sozialisation in Deutschland über unterschiedliche Medienkanäle, politische Einflussnahmen und Beziehungen, über familiäre Traditionen und Prägungen oder in den Ferien, trotz geografischer Distanz, intensiv erfahren wird.

Die kritisierte, gleichwohl immer noch virulente Vorstellung einer unumgänglichen Assimilation im klassischen Sinne einer stufenweisen Anpassung der Zugewanderten und ihrer Nachfahren an die aufnahmegesellschaftlichen Kulturen und Strukturen und einer dafür notwendigen Aufgabe der Herkunftskultur als Gewährleistung erfolgreicher Integration (vgl. Esser 1999: 21, 2001: 19, 2009: 362; Bade/Bommes 2004) wird durch die Ergebnisse nicht gespiegelt. Eher kann mit Blick auf Heimatgefühle und Loyalitäten von identitätsbezogenen MehrfachVerortungen, Mehrfach-Mentalitäten oder Mehrfach-Zugehörigkeiten ausgegangen werden, die in (kollektiven) Sozialisations- und Gruppenbildungsprozessen entstanden sind und die unterschiedliche Lebensperspektiven umfassen. ${ }^{133}$ Diese können

${ }^{133}$ Für die Individual- und Organisationsebene akzentuieren dies theoretische Literatur und empirische Studien. Siehe dazu u. a.: Mecheril 2003; Berry et al. 2006; Bertelsmann Stiftung 2009; Fincke 2009; Aicher-Jakob 2010; Hans 2010; Maehler 2012; Mehdi 2012; Foroutan 
Spannungsverhältnisse zwischen erforderlichen und eingeforderten Anpassungen und kulturellen Selbstbehauptungen und damit auch Loyalitätskonflikte provozieren. Deren Austragung ist ein Prozess, der sich im Generationenverlauf vollzieht und der unter jüngeren Generationen und Organisationen in der Regel immer mehr die Orientierung an der deutschen Gesellschaft als bevorzugter Maßstab und kulturübergreifende Ausrichtungen erkennbar werden lässt.

In einer pluralistischen Einwanderungsgesellschaft wie Deutschland sind die Anerkennung von nebeneinander bestehenden unterschiedlichen Interessen sowie kulturellen Besonderheiten, ein respektvolles Miteinander und wechselseitige Verständigungsprozesse wichtige Voraussetzungen für die Konsolidierung intakter sozialer Beziehungen und Identifikationen mit vorgefundenen Referenzrahmen. Dies gilt nicht allein für Migrationsbezüge und Allochthone, sondern auch für Autochthone. Von circa jeweils einem Viertel der MSO werden gesellschaftliche Anfeindungen und Fehlplatzierungen wahrgenommen, was für religiöse Vereinigungen in einem höheren Ausmaß zutrifft (7.1.7). Auch anhand der Regressionen konnten bezüglich der Perzeption von Fremdenfeindlichkeit und Diskriminierung signifikante Effekte in Bezug auf nach innen gerichtete, religiöse und nicht-religiöse Aktivitäten festgestellt werden. Die in diesem Zusammenhang angestellte Vermutung, dass abwertendes, fremdenfeindliches Verhalten und Gedankengut die Konzentration auf die Herkunftskultur und die eigene Gruppe forcieren kann, ist plausibel. In jedem Fall ist Fremdenfeindlichkeit als eine Begleiterscheinung der Aktivitäten (nicht-)religiöser MSO einzustufen. Damit werden hinsichtlich der gegenseitigen Akzeptanz und des Abbaus von Vorurteilen Verbesserungsund Handlungsbedarfe deutlich, deren Notwendigkeit - zumal angesichts der für Deutschland ermittelten Stabilität von fremden-, islam- und demokratiefeindlichen Einstellungen und Tendenzen (vgl. u. a. Zick et al. 2019a; Pollack et al. 2014) - umso stärker unterstrichen werden muss.

\section{Produktion religiösen Sozialkapitals ist differenziert und flexibler zu denken}

Die Charakterisierung und Einordnung der unterschiedlichen Konfessionen im Rahmen der theoretischen Überlegungen zur Generierung von abgrenzenden oder gesellschaftsorientierten Sozialkapitalformen (II. 4.2.4) können mit dieser Arbeit nicht bestätigt werden. Insofern könnte hier vermutet werden, dass das prosoziale Engagement der Vereinigungen auf einer verallgemeinerbaren religiösen Motivation

2013; Scheer 2014; Canan 2015; Kazzazi et al. 2016; Freist et al. 2019; für (migrantische) Organisationen u. a.: Pries 2010a, b; Pries/Sezgin 2010a, 2012; Nagel 2013b; Beilschmidt 2016; Polak/Reiss 2015. 
beruht und nicht auf unterschiedliche inhaltliche Glaubensausrichtungen zurückzuführen ist (Liedhegener 2016). Dies soll aber nicht als Nivellierung der religiösen Traditionen verstanden werden. Die Varianzen zwischen und innerhalb der einzelnen Konfessionen, die auch auf die organisationale Binnenheterogenität zurückzuführen sein sollten, sind aufschlussreich (vgl. 7.2.2; 7.2.5); wenngleich sie aufgrund der zum Teil sehr geringen Fallzahlen nicht als repräsentativ veranschlagt werden können, lassen sie insgesamt auf ein innerhalb von religiösen Traditionen vorhandenes Kontinuum, ein Mehr-oder-Weniger bzw. Sowohl-als-Auch, schließen.

Der für die Muslime geltend gemachte ,kollektivistische[] Familismus [...], welcher dem verwandtschaftlichen Zusammenhalt höchste Priorität einräumt“" (Traunmüller 2012: 59) und entsprechend in einem starken Bonding resultieren sollte, lässt sich anhand der Daten explizit nicht nachweisen. Dies bedeutet nicht, dass es diesen kollektivistischen Familismus nicht gibt. Er kann durchaus existent sein und das Bonding nach innen stärken. Die Relevanz einer intensiven Einbindung von ganzen Großfamilien ist im Rahmen der Auswertung beispielsweise bei den Strategien der Mobilisierung von Engagierten geltend gemacht worden (7.1.2.4). Eine ausschließliche Konzentration auf Familienverhältnisse lässt sich allerdings mit den auf Organisationsebene erhobenen Daten nicht belegen und nicht als ein speziell für Muslime geltendes Phänomen ausmachen. Die Ergebnisse sprechen eher dafür, dass die familialen kollektiven Bindungen zivilgesellschaftlich wirksame Kräfte implizieren bzw. freisetzen. Angesichts der Befunde und ihrer hier erfolgenden Auslegung ist gleichwohl nicht zu verkennen, dass Religionen bzw. kollektive religiöse Identitäten durchaus stark abgrenzende Verhaltensweisen hervorbringen können; dieses konnte mit der vorliegenden Studie aber nicht eingefangen werden.

Um die Tragweite der Ergebnisse zu den religiösen MSO ermessen und in dieser Studie ggf. unsichtbar gebliebene Nuancierungen ermitteln zu können, wäre die Analyse einer größeren Stichprobe zweifelsohne erkenntniserweiternd. In Hinblick auf die hier zu größeren übergeordneten Religionsgruppen zusammengefassten kleineren Gruppen und Strömungen könnte eine Ausdifferenzierung der Ermittlung dienlich sein, ob und inwieweit z. B. innerislamische Ausrichtungen eigenständige zivilgesellschaftliche Performanzen zeigen.

\section{Bonding und bridging als mehrfach bedingte Phänomene}

Eine hier zu stellende, zentrale Frage lautet, wieso Erklärungen für organisationale Ausrichtungen und Handlungsformen einfach ausfallen sollten: Es erscheint zu simpel, zu komplexitätsreduzierend, Bridging- und Bonding-Ausprägungen in essentialisierender Manier allein an für spezifische Konfessionen universell geltend gemachten Kollektivmerkmalen festzumachen (vgl. dazu II. 4.2.4). Anhand der 
Darlegung des Zusammenspiels von bonding und bridging (7.2.2), der unter Einbezug mehrerer relevanter Variablen vorgenommenen Typenbildung (7.2.4) wie auch der statistischen Clusteranalyse (7.2.5.3) kann aufgezeigt werden, dass der Charakter der religiösen Vergemeinschaftungen in Bezug auf bonding/bridging nicht eindeutig zu bestimmen ist. Vielmehr zeigen sich Mischformen. Diese inhärenten Ambivalenzen wurden in dieser Arbeit auch theoretisch beleuchtet (II. 4.2.5): Sind religiöse Vereinigungen einerseits besonders, anfällig' für Binnenorientierungen, sind sie andererseits aufgrund prosozialer Normen und sittlicher Weisungen (z. B. Nächstenliebe), gottgefälliger Handlungsweisen etc. quasi prädestiniert dafür, sich um Angehörige anderer Gruppen zu kümmern. Insofern zeigt sich - wie im KONIDSurvey 2019 - auch in dieser Studie, dass „Religionsgemeinschaften keine in sich homogenen Gebilde sind, sondern in einem variierenden Ausmaß Anteil an ihrer pluralen gesellschaftlichen Umwelt haben“" (Liedhegener et al. 2019: 46).

Bridging und bonding sind nicht nur durch religiös verankerte Mentalitäten induziert, sondern von mehrfach bedingten Haltungen, Überzeugungen und Motiven, die die beteiligten Individuen veranlassen, bestimmte Interessen in die Tat umzusetzen oder dies nicht zu tun. Bridging und bonding lassen sich multifaktoriell, nicht allein mit religiösen Orientierungen oder Ressourcenausstattungen erklären. Dieses haben auch die Ergebnisse der multiplen Regressionen verdeutlicht. Angesichts ähnlich zu verortender Ergebnisse sehen die Autoren des KONID-Forschungsberichtes 2019 in diesen ,einen empirischen Anhaltspunkt für die emergenten Eigenschaften sozialer Systeme [...]. Gesamtgesellschaftliche Phänomene [...] beruhen auch auf Effekten, die sich erst durch die Kombination einer Vielzahl von Eigenschaften einstellen und dadurch eine eigenständige Qualität und Bedeutung erhalten“ (Liedhegener et al. 2019: 46 f.).

Bridging und bonding realisieren sich fallspezifisch in einem Mischungsverhältnis verschiedener Einflussfaktoren und Situationsumstände. Sie werden u. a. bestimmt von Zielsetzungen, Selbstverständnissen und Strukturen der MSO, der Dauer ihres Bestehens, von ihrem Verhältnis zum Herkunfts- und Aufnahmeland, ihrer jeweiligen ethnischen Zusammensetzung, von Opportunitätsstrukturen, Wechselwirkungen mit Akteuren in ihren Umwelten und natürlich auch deren Verhaltensweisen (vgl. dazu Nagel 2013a, 2015a; Geißel et al. 2004: 12; Rauer 2004: 225 f.; Diehl 2004: 248; Berger et al. 2004; Kap. I. 2.2; II. 4.2.3).

Die Ergebnisse der quantitativen und typisierenden Analysen vorliegender Studie korrespondieren folglich mit dem auf Basis qualitativer Fallstudien für unterschiedliche Migrantengemeinden in NRW konstatierten Befund, dass keine eindeutigen Kausal- und Wirkungsrichtungen von Einflussfaktoren, sondern in Bezug auf Aktivitäten und Vernetzungsverhalten vielmehr von einem „Ursachenbündel“ zu 
sprechen und eine ,zentrale Stellschraube zur Aktivierung der zivilgesellschaftlichen Potentiale religiöser Migrantengemeinden“ (Nagel/Plessentin 2015: 262) entsprechend nicht zu identifizieren sei (vgl. I. 2.2).

An dieser Stelle ist jedoch in Bezug auf die im Kontext der Typenbildungen unter die Lupe genommenen Organisationsmerkmale einschränkend zu vermerken, dass die personelle Ressourcenausstattung hinsichtlich der Ermöglichung von vielfältigen Aktivitäten bei den Bonding/Bridging- sowie Bridging IV-Typen als eine relevante Einflussgröße einzustufen ist, die als solche aber nicht durchgängig sichtbar wird (7.2.5). Insofern lässt sich nicht feststellen, dass es ohne Hauptamtliche kein Bridging gibt oder - umgekehrt - dass, wenn Bridging-Ausprägungen vorhanden sind, dass dann auch Hauptamtliche verfügbar sind.

\section{Hohe integrationspolitische Erwartungshaltungen gegenüber MSO}

Bonding-Performanzen können intendiert und nicht-intendiert sein, wie sich beispielsweise anhand des Typus Bonding III, den ,eigeninteressengeleiteten Kulturund Freizeitvereinen“, nachweisen lässt. Zum Vergleich kann man sich vor Augen führen, dass die Pflege kultureller Eigenheiten und Interessen in nicht-migrantischen Vereinigungen nicht mit bewussten Rückzugs- oder Abgrenzungstendenzen assoziiert wird. Die einst Zugewanderten und ihre Nachfahren haben in der normativ aufgeladenen und mit Verpflichtungen konnotierten Integrationsdebatte eine Bringschuld zu leisten. Dabei ist nachvollziehbar, dass ausschließliche Bonding-Ausrichtungen als solche integrationspolitischen Belangen und der gesellschaftlichen Einfügung entgegenstehen. Jedoch soll hier - wie auch zu Beginn des Kapitels (7.2) - argumentiert werden, dass migrantische Bonding-Vereine nicht automatisch desintegrative Tendenzen produzieren, wenn sie die vielfach eingeforderte Integrationsarbeit nicht erfüllen. In diesem Zusammenhang ist auch zu bedenken, dass eine (ostentative) Sichtbarmachtung zivilgesellschaftlich bedeutungsvoller Leistungen, um ggf. Fördergelder zu akquirieren, eigenkulturellen Selbstverständnissen und habituellen Mentalitäten entgegenstehen kann.

\section{Bonding-Zusammenschlüsse in Studie unterrepräsentiert}

Einschränkend sei an dieser Stelle darauf hingewiesen, dass im Rahmen dieser Studie MSO, die starke Bonding-Tendenzen aufweisen, kaum für die Beteiligung an der Befragung gewonnen und daher nicht abgebildet werden konnten. An einer Befragung, wie sie dieser Arbeit zugrunde liegt, werden in erster Linie aktive und interessierte Organisationen teilnehmen, die ihre Arbeit sichtbar machen wollen. Auch sind Lippenbekenntnisse bzw. sozial erwünschtes Antwortverhalten zwecks Errichtung einer „Legitimationsfassade“ nicht grundsätzlich auszuschließen (II. 
4.5). Insofern ist hinsichtlich der Datenerhebung von einem Bias auszugehen, der hinsichtlich der Ergebnisse kritisch zu reflektieren ist (5.3). Diese mögliche Verzerrung verweist auf das grundsätzlich notwendige Nachdenken über alternative, die quantitative Forschung flankierende Erhebungsdesigns, wie etwa mehrsprachigen Face-to-Face-Befragungen. Mit diesen könnten unter Einbezug von ,türöffnenden“ Personen, die in den jeweiligen Communities Vertrauen genießen, auch schwerer zugängliche MSO für eine Teilnahme gewonnen werden, die mit postalischen, telefonischen oder Online-Befragungen kaum zu erreichen sind.

Dass es Bonding-Organisationen gibt, kann anhand des Typ Bonding I den „Selbstbezogenen“ - aufgezeigt werden. Ihre Unterrepräsentation lässt sich sicherlich unterschiedlich begründen: Starke politisierte und von außen kritisch beobachtete Auseinandersetzungen innerhalb islamischer Verbände, eine mit Introvertiertheit einhergehende argwöhnische Distanznahme oder auch spezifische (religiöse) Mentalitäten, die eine Teilnahme an Befragungen solcher Art grundsätzlich ablehnen und dies der Studienleiterin per E-Mail oder Telefon zum Teil auch mitteilten (5.3).

Was im Rahmen dieser Arbeit ebenfalls nicht eingefangen wurde, sind unzivile, demokratiegefährdende Formen migrantischen Engagements sowie erstarkende nationalistische Orientierungen junger Türkeistämmiger der dritten und vierten Generation, die insbesondere in NRW in den letzten Jahren auffällig geworden sind und die zu Beginn dieses Auswertungskapitels (7.2) bereits erwähnt wurden. Sie stehen in Zusammenhang mit den politischen Entwicklungen in der Türkei und deren grenzüberschreitenden Auswirkungen, insbesondere aufgrund der türkischen „Diaspora“-Politik, mit der Einfluss auf die in Deutschland bestehenden Strukturen der türkeistämmigen Community genommen werden soll (Sauer 2018: 7 f., 11 f.). Sie sorgen auf kommunalpolitischer Ebene in NRW für Irritationen, da die Haltungen pro Erdoğan Widerstand gegenüber den demokratischen und rechtsstaatlichen Bedingungen in Deutschland implizieren, unter denen die Jugendlichen aufgewachsen sind und deren Tragweite und Nutzen sie daher eigentlich bevorzugt wertschätzen sollten. ${ }^{134}$

Wenngleich die Befunde dieser Arbeit für einen Ausschnitt der MSO-Landschaft in NRW im Großen und Ganzen erfolgreiche Integrationsprozesse spiegeln, weisen gegenwärtig virulente Phänomene darauf hin, dass Prozesse von Integration und Beheimatung immer auch gegenläufige, spannungs- und konfliktbeladene Prozesse zeigen und nicht geradlinig oder unilokal verlaufen. Vor dem Hintergrund komplexer

${ }^{134}$ Diese Aussage basiert auf Informationen, die die Autorin 2017/18 im Rahmen der Feldsondierung sowie des Besuchs von kommunalen Netzwerktreffen, Migrationskonferenzen und Integrationsratssitzungen in einzelnen der Erhebungskommunen in NRW gewinnen konnte. 
sozialer, politischer, kultureller Verflechtungen ist Integration damit immer als ein mehrdimensionaler, ergebnisoffener sozialer Prozess zu betrachten, der die gesamte Gesellschaft betrifft und v. a. die gegenseitige Akzeptanz der involvierten Menschen als eine wichtige Voraussetzung hat.

\section{Notwendigkeit von wissenschaftlicher Objektivität und Kontextsensibilität}

In diesem Zusammenhang ist angesichts der theoretischen Annahmen zur Produktion von Sozialkapital in unterschiedlichen Konfessionen bzw. religiösen Gemeinschaften abschließend kritisch zu reflektieren, dass Wissenschaftler durch normativ gefärbte, standortgebundene Konzeptualisierungen selbst Etikettierungsprozesse provozieren und Schwarzweiß-Kategorisierungen zu Bezeichnungskategorien werden lassen können, die v. a. in sozialwissenschaftlichen Fachdisziplinen als für Konfliktsituationen und Wahrnehmungsstörungen ursächlich durchleuchtet werden. Denn das auf eindeutige Charakterisierungen setzende Schubladendenken basiert auf unzulässigen Homogenisierungen und Essentialisierungen und damit Vereinfachungen komplexer Erfahrungszusammenhänge und Zugehörigkeitsmodi der in den religiösen Gemeinschaften involvierten Personen. Darüber hinaus sind die Annahmen nicht kontextsensitiv, was besonders wichtig ist, wenn gesellschaftspolitische Realitäten sachlich-differenziert entlang mehrerer relevanter Einflussfaktoren analysiert werden sollen. Insofern ist auch die zum In-Kategorien-Denken veranlasste Wissenschaft aufgerufen, oktroyierte Zuschreibungen als solche zu erkennen und offen zu sein für variierende Ausprägungen von religiösem Sozialkapital. Denn Angehörige religiöser und säkularer Gemeinschaften sind unterschiedlichen lebensweltlichen sozialen, politischen, kulturellen, ökonomischen Referenzrahmen ausgesetzt. Wie ausgeführt wurde, beziehen sich Zugehörigkeitsgefühle damit nicht notwendigerweise nur auf ein Land oder eine religiöse Tradition, sondern es bestehen vielmehr mehrere Zugehörigkeitsanker.

Aufgrund der verschiedenen Analyseebenen und methodischen Verfahren zur Untersuchung von bonding und bridging fallen die Ergebnisse unterschiedlich aus. Dadurch zeigt sich, wie sehr Ergebnisse von jeweils gewählten Analysemethoden abhängen. Dies verdeutlicht wiederum das Risiko, dass Schlussfolgerungen als grundsätzlich geltende Muster im Diskurs zementiert werden. Daher soll abschlieBend für eine subtile, nicht pauschalisierende Betrachtung und sorgfältige Auswahl der Untersuchungsmethoden plädiert werden.

Ein Anliegen der Autorin ist es, mit der in diesem Kapitel schrittweise, methodisch differenziert und detailliert vollzogenen empirischen Analyse von Bridging- und Bonding-Performanzen migrantischer Organisationen in der Summe 
zur Versachlichung einer nach wie vor auch polarisiert und polemisch geführten Integrationsdebatte beizutragen.

\subsection{Zusammenfassung der zentralen Ergebnisse, Fazit und Ausblicke}

Wenn im Folgenden die wesentlichen Befunde und daraus resultierende Schlussfolgerungen aufgeführt werden, dann geschieht dies im Interesse kondensierter Resultatsicherung. Die übersichtliche Zusammenführung zentraler Ergebnisse des ersten großen Auswertungsteils (III. 7.1) ist das wichtigste Anliegen dieses Schlusskapitels. In den Blick genommen werden Gründungsjahre der MSO, Mitglieder und Räumlichkeiten, finanzielle Quellen und Ressourcen, die Verfügbarkeit von Hauptamtlichen und Engagierten, deren zahlenmäßige Entwicklung seit 2013 sowie Strategien der Mobilisierung und Motive freiwillig Engagierter. Von großer Relevanz sind die vielschichtigen Organisationsziele, die Selbstverständnisse der MSO, das soziale Miteinander in der Organisationsinnenwelt sowie Tätigkeitsbereiche und Zielgruppen der Vereinigungen. Schließlich werden spezifische Aktivitäten in der Flüchtlingshilfe, der Aktivitätsradius der MSO, deren Kooperationsverhalten sowie die Einschätzung der eigenen Arbeit und Situation dargelegt. In einem abschließenden Teil werden noch einmal die Unterschiede und Gemeinsamkeiten zwischen religiösen und säkularen MSO - das zentrale Thema dieser Arbeit - aufgegriffen und mit Blick auf ihre theoretischen wie lebenspraktischen Implikationen reflektiert. Ebenfalls werden weitere thematische und methodische Perspektiven der Erforschung von MSO aufgemacht.

An dieser Stelle ist abermals darauf hinzuweisen, dass die Ergebnisse auf einer schriftlichen Befragung von Funktionsträgern (v. a. Vorstandsvorsitzenden) basieren, die mit ihrer Organisation sehr gut vertraut sind. Die Funktionsträger haben als Repräsentanten der MSO Auskunft über ihr „Betriebswissen“ gegeben. Es handelt sich somit um durch die Person eines Funktionsträgers gefilterte und kommunizierte Informationen zur organisationalen Performanz (6.1). Die Frage, ob die Ergebnisse als für die Grundgesamtheit der MSO in NRW repräsentativ einzustufen sind, kann aufgrund fehlender Kenntnisse der entsprechenden Merkmale in der Grundgesamtheit nicht beantwortet werden (5.3).

Gleichermaßen ist mit Blick auf die Ergebnisse für die religiösen Vereinigungen noch einmal herauszustellen, dass islamische MSO in der Substichprobe der religiösen MSO $\left(n_{R}=80\right)$ mit 44 Fällen dominant vertreten sind. Die Überrepräsentation entspricht durchaus auch dem für NRW festzustellenden Gesamtbild 
(I. 2.1). Darüber hinaus haben 12 alevitische, 17 christliche, 3 jüdische, 3 ezidische sowie eine hinduistische Vereinigung an der Befragung teilgenommen.

\section{Gründungsjahre}

- Die religiösen MSO sind signifikant älter

Die Gründungsjahre der MSO sind sehr breit gestreut (1947 bis 2018). 74 Prozent der befragten MSO wurden in den letzten 29 Jahren (1991 bis 2018) gegründet. Die religiösen MSO sind signifikant älter als die säkularen.

\section{Mitglieder}

- Die religiösen MSO haben signifikant mehr Mitglieder homogenerer Provenienz Jeweils ein Drittel und damit die meisten der MSO haben 11 bis 50 (33\%) oder mehr als 100 Mitglieder (33 \%). Religiöse MSO haben signifikant mehr Mitglieder. In circa der Hälfte der MSO kommen die Mitglieder aus einem Herkunftsland, wobei es sich bei circa der Hälfte der herkunftslandhomogenen Organisationen um die Türkei handelt. Die Herkunftslandhomogenität ist mehrheitlich für die religiösen MSO auszumachen (RMO: 62 vs. MO: $42 \%$ ) $^{135}$; darüber hinaus zeigt sich bei 50 Prozent der religiösen MSO eine doppelte Homogenität durch die Koinzidenz von herkunftslandbezogener und konfessioneller Konformität der Mitglieder.

Jenseits einer für die Hälfte der MSO festzustellenden Herkunftslandhomogenität zeigen sich in den anderen MSO ethnische Durchmischungen und Pluralität. Neben den Türkeistämmigen sind afrikanische und andere Communities bedeutsam, die zukünftig in der Forschung mehr Aufmerksamkeit erhalten sollten. Angesichts der Ergebnisse lässt sich vermuten, dass MSO in besonderer Weise fähig sind, Rahmenbedingungen zu schaffen, um Menschen unterschiedlicher Nationalitäten zu inkorporieren und mit Blick auf interkulturelle Öffnungs- und Lernprozesse als Beispiele guter Praxis zu fungieren.

${ }^{135}$ Zur Ausweisung von zu einem jeweiligen Organisationstypus (nicht-/religiös) gehörigen Prozentwerten werden die Kürzel RMO (für religiöse) und MO (für nicht-religiöse) verwendet (6.2). 
- Konfessionelle Homogenität und konfessionelle Melange der Mitglieder

Für 38 Prozent der MSO lässt sich eine konfessionelle Homogenität feststellen. Diese trifft auf 73 Prozent der religiösen MSO und auf 20 Prozent der nichtreligiösen MSO zu, und zwar bei beiden Typen mehrheitlich für den Islam bzw. das Sunnitentum.

Wie auch bei der Ermittlung der Herkunftsländer spiegeln die Ergebnisse zum einen eine insbesondere für religiöse MSO zu erwartende konfessionelle Homogenität der Mitglieder, zum anderen aber auch eine Mischung unterschiedlicher Glaubensrichtungen. Neben der islamisch-sunnitischen und alevitischen Tradition sollten zukünftig in der Forschung gezielt auch andere und in Deutschland und NRW quantitativ weniger vertretene religiöse migrantische Selbstorganisationen - hinduistische, orthodoxe, jüdische - soziologisch untersucht werden (vgl. z. B. Nagel 2015a).

Die Ergebnisse zeigen zum einen, dass religiöse MSO in Bezug auf die Glaubensrichtung der Mitglieder nicht immer eine homogene Struktur aufweisen; zum anderen verdeutlichen sie, dass Religion bzw. eine Affinität zu religiösen Glaubensrichtungen auch in ,säkularen“ MSO eine Rolle spielen können.

\section{Räumlichkeiten}

- Die religiösen MSO verfügen signifikant häufiger über Räumlichkeiten

76 Prozent der MSO stehen Räumlichkeiten zur Verfügung, wobei dies für religiöse MSO signifikant häufiger zutrifft. $\mathrm{Zu}$ erwähnen ist an dieser Stelle, dass die Funktionsträger in dem am Ende des Erhebungsbogens für Anmerkungen und Kommentare vorgesehenen Platz häufiger auf die Problematik verwiesen, dass ihre Organisationen über keine eigenen oder adäquaten Räumlichkeiten verfügten. Diese missliche Lage erschwere die Umsetzung von Aktivitäten und geplanten Programmen maßgeblich (s. auch II. 4.3.2; vgl. auch Friedrichs et al. 2020: 89 f.).

\section{Finanzielle Quellen und Ressourcen}

- Religiöse MSO: Mit weniger öffentlichen Finanzierungsquellen vermögender

Die MSO finanzieren sich überwiegend über Mitgliedsbeiträge (76\%) sowie über Spenden und Sponsorengelder (67\%), wobei sich in Bezug auf diese Quellen jeweils eine signifikant größere Bedeutung für die religiösen MSO ergibt. Fördermittel von Stadt und Bund sowie insbesondere vom Land Nordrhein-Westfalen stellen hingegen für die säkularen Vereinigungen relevantere Subventionen dar. 
Während die säkularen MSO auf eine signifikant größere Anzahl von Finanzierungsquellen zurückgreifen können, sind die religiösen MSO mit weniger öffentlichen Subventionen hinsichtlich des per Anno verfügbaren Finanzvolumens insgesamt vermögender. Zudem sind mitgliederreiche MSO signifikant finanzstärker: Je mehr Mitglieder eine MSO hat, desto mehr Geld steht ihr pro Jahr zur Verfügung.

Die Ergebnisse spiegeln zum einen Präferenzen politischer Förderung von MSO auf Bundes-, Landes- und kommunaler Ebene in NRW wider (Klie 2020). Wenngleich in NRW jeder Verein mit MSO-Eigenschaften und integrativen Tätigkeiten gefördert werden kann, lässt sich aufgrund registrierter Radikalisierungstendenzen religiöser MSO sowie anhaltender, nicht tolerierter politischer Einflussnahmen aus dem Ausland eine zunehmende Verunsicherung und Skepsis feststellen. Dies kann für die misstrauisch beäugten MSO bedeuten, dass kommunale Förderungen beendet oder sie von solchen ausgeschlossen werden.

Zum anderen ist in Erwägung zu ziehen, dass bei religiösen Vereinigungen der Wunsch nach bzw. Bedarf an politischen Fördermitteln weniger stark ausgeprägt ist und entsprechend wenig(er) Förderanträge gestellt werden und dass nicht-religiöse MSO, die mehr hauptamtliches Personal und fachliche Professionalisierung vorweisen, bei der Akquise von Fördermitteln einen strukturellen Vorteil besitzen (vgl. Friedrichs et al. 2020: 75 f.).

46 Prozent der MSO geben an, finanzielle Engpässe zu haben. Hier zeigen sich in Bezug auf eine stärkere Relevanz für religiöse oder säkulare MSO keine signifikanten Ergebnisse. Die Gruppe der MSO, die finanzielle Engpässe bekundet, verfügt pro Jahr zumeist auch über weniger Geld.

\section{Hauptamtliche und Engagierte}

- Die säkularen MSO haben signifikant mehr Hauptamtliche

33 Prozent der MSO geben an, hauptamtliche, bezahlte Mitarbeiter zu haben ${ }^{136}$. Hauptamtliche sind in den meisten MSO (48 \%) mit einer Anzahl von 2 bis 10 Personen und mit einer Person in 35 Prozent der Vereinigungen vertreten. Die säkularen MSO verfügen über signifikant mehr Hauptamtliche. Das Vorhandensein von bezahlten Hauptamtlichen verweist auf den Spezialisierungs- und Professionalisierungsgrad der Organisationen (II. 4.3.4), der somit insbesondere für die säkularen höher ausfällt (vgl. auch Halm et al. 2020: 119).

${ }^{136}$ Im Vergleich dazu wurde dies im Rahmen des ZiviZ-Surveys 2017 für 23 Prozent der MSO festgestellt (Priemer 2017: 3; I. 2.1). 
In 93 Prozent der MSO übernehmen freiwillig Engagierte Aufgaben. Die Anzahl von Engagierten kumuliert mit 56 Prozent deutlich bei 1 bis 10 Personen. Es ist ein proportionales Verhältnis zwischen Mitglieder- und Engagiertenzahlen festzustellen: Je höher die Mitgliederzahlen in einer MSO ausfallen, desto mehr Engagierte sind dort vorzufinden.

\section{- Die religiösen MSO haben signifikant mehr Engagierte}

Zudem zeigt sich, dass die religiösen MSO über signifikant mehr Engagierte verfügen. Diesbezüglich wurde argumentiert, dass die Gewinnung von mehr Freiwilligen innerhalb der religiösen MSO mit den dichten Gemeindestrukturen und den dort vermehrt stattfindenden Interaktionen, aber auch einem höheren Bedarf zu erklären sein sollte. Inwieweit die Vermutung zutrifft, dass die religiösen Vereinigungen hinsichtlich der Rekrutierung von Engagierten erfolgreicher sind als die säkularen, wurde im Weiteren gesondert mit der Hypothese $\mathbf{4}_{\mathbf{1}}$ überprüft.

- Hauptamtliche gewährleisten breiteres Kooperationsspektrum

Geprüft wurde, ob zwischen der Verfügbarkeit von personellen Ressourcen (Mitglieder, Hauptamtliche, Engagierte) und als ausreichend empfundenen finanziellen Ausstattungen einerseits sowie der Leistungsfähigkeit der Vereinigungen andererseits proportionale Zusammenhänge bestehen. Angenommen wurde, dass ressourcenstärkere MSO eine höhere Anzahl von Aktivitäten und ein größeres Spektrum an Kooperationspartnern aufweisen (Hypothese H2). Die Ergebnisse multipler Regressionen haben gezeigt, dass Einrichtungen mit einer höheren Anzahl an Hauptamtlichen auch signifikant mehr Kooperationspartner aufweisen. Zudem ist der Einfluss von Einrichtungen mit 11 bis 50 Mitgliedern im Vergleich zu jenen mit höchstens 10 Mitgliedern auf die Anzahl der Kooperationspartner signifikant größer. Hinsichtlich des Ausmaßes der Aktivitäten der MSO zeigten sich die Zahlen der Hauptamtlichen sowie Mitglieder hingegen nicht als signifikante Einflussgrößen. ${ }^{137}$ Darüber hinaus sind eine als (nicht) hinlänglich empfundene finanzielle Ressourcenausstattung sowie die Verfügbarkeit von Engagierten für das Leistungsspektrum von MSO nicht ausschlaggebend.

${ }^{137}$ Im Zusammenhang mit den für das Aktivitätsspektrum der MSO (als abhängige Variable) im betreffenden Regressionsmodell komplett nicht signifikanten Ergebnisse wurde kritisch angemerkt, dass aufgrund eines sozial erwünschten und/oder eines die Aktivitäten der MSO überschätzenden Antwortverhaltens eine valide Verhältnismäßigkeit zwischen der Anzahl der Aktivitäten und der Anzahl der Hauptamtlichen (bzw. weiterer unabhängiger Variablen) für die Berechnung der Regressionen ggf. nicht gegeben ist (s. dazu 7.1.6). 


\section{Entwicklung der Zahlen von Mitgliedern, Hauptamtlichen und Engagierten seit 2013}

- Signifikanter Anstieg von Hauptamtlichen in den säkularen MSO

Hinsichtlich der Anzahl der Mitglieder, Hauptamtlichen und freiwillig Engagierten lässt sich für alle drei Statusgruppen seit dem Jahr 2013 sowohl Stabilität als auch Anstieg feststellen. Letzterer fällt für die Mitglieder (47 \%) am größten und größer aus als die Stabilität (36\%).

Auch bei den hauptamtlich Beschäftigten ist die Zunahme größer (51\%) als die Konstanz der Zahlen (44\%). Allerdings gilt der Anstieg im signifikanten Ausmaß insbesondere für die nicht-religiösen Organisationen (MO: 65 \% vs. RMO: $24 \%$ ). Dies verweist auf deren professionelle Ausrichtung und einen Professionalisierungstrend (vgl. auch Halm et al. 2020: 119; Friedrichs et al. 2020: 45). In den religiösen MSO sticht hingegen die Stabilität der Anzahl Hauptamtlicher hervor (76\% vs. $27 \%$ ); zudem wird in diesen kein einziger Rückgang vermerkt. Eine Zunahme der freiwillig Engagierten trifft insbesondere für die religiösen MSO zu (RMO: $42 \%$ vs. MO: $30 \%$ ), allerdings nicht in einem statistisch signifikanten Ausmaß.

\section{Mobilisierung der freiwillig Engagierten}

- Die religiösen MSO sind in puncto Rekrutierung von Ehrenamtlichen erfolgreicher

Bei der Einschätzung der Aussagen zu den freiwillig Engagierten wurde deutlich, dass sich mehrheitlich genügend Freiwillige gewinnen (61\%) und sich ehrenamtliche Leitungspositionen bei 51 Prozent der MSO gut besetzen lassen. Bei den anderen MSO (39\% und $49 \%$ ) ist diesbezüglich von Schwierigkeiten oder Unzufriedenheit auszugehen.

Die religiösen MSO können in einem höheren Ausmaß genügend Engagierte (RMO: $70 \%$ vs. MO: $58 \%$ ) sowie ausreichend Personen für die Ehrenämter aktivieren (69 \% vs. $44 \%$ ). Allerdings ist nur in Bezug auf die Besetzung von ehrenamtlichen Leitungspositionen für die religiösen Vereinigungen eine signifikant bessere Situation zu ermitteln. Angesichts der statistischen Testergebnisse kann der erste Teil der Hypothese 4 ( $\left.\mathrm{H}_{4}{ }_{1}\right)$, mit dem formuliert wurde, dass religiöse MSO in puncto Freiwilligengewinnung erfolgreicher sind als nicht-religiöse, folglich nur in Bezug auf die Gewinnung von Personen für Ehrenämter bestätigt werden. In diesem Zusammenhang ist jedoch - wie oben dargelegt - zu berücksichtigen, dass für die religiösen MSO hinsichtlich der Engagierten eine signifikant größere Anzahl und ebenfalls eine (nicht signifikante) Zunahme der Anzahl von Freiwilligen zu 
konstatieren ist. Daher kann durchaus geschlussfolgert werden, dass die religiösen MSO in dem Bestreben, mehr Freiwillige zu gewinnen, positivere Resultate erzielen. Die Ergebnisse verdeutlichen darüber hinaus, dass Ehrenamt und Engagierte in den religiösen MSO die tragenden Kräfte sind. Aufgrund ihrer fundamentalen Bedeutung ist die Rekrutierung essenziell und den Angaben der Funktionsträger zufolge scheinbar noch zu verstärken.

- Trend zum Interesse an zeitlich befristeten Engagements

Darüber hinaus bestätigen 68 Prozent der MSO, dass die Engagierten in der Mehrzahl an zeitlich befristeten Tätigkeiten interessiert sind (MO: $72 \%$; RMO: $60 \%$ ). Die Ergebnisse spiegeln damit die in der Forschung festgestellten Trends, dass unter Engagierten ein Interesse an unverbindlicher Übernahme kurzfristiger Tätigkeiten vorherrscht und in puncto Gewinnung von Ehrenamtlichen Schwierigkeiten bestehen (BMFSFJ 2017: 136 ff.; Friedrichs et al. 2020: 21). Hier ist einschränkend zu vermerken, dass sich die Ausgangslage für die Rekrutierung von Ehrenamtlichen bei den religiösen Vereinigungen besser darstellt.

- Freiwilligengewinnung: Hohe Relevanz von persönlicher Ansprache, motivierenden Personen und Veranstaltungen

Hinsichtlich der Frage, auf welche Art und Weise Freiwillige für die Übernahme unbezahlter Aufgaben erreicht und gewonnen werden können, zeigt sich, dass Freiwillige in 93 Prozent der MSO insbesondere durch die Ansprache von Freunden und Bekannten, durch eine oder mehrere motivierende und begeisternde Person(en) $(85 \%)$ oder mit regelmäßigen Veranstaltungen (79 \%) mobilisiert werden können.

Im Gesamtbild sind anhand einer Explorativen Faktorenanalyse (EFA) in Bezug auf die insgesamt acht vorgegebenen Mobilisierungsmaßnahmen drei Faktoren zu identifizieren:

Mobilisierung von Engagierten durch

1) Öffentlichkeitsarbeit, deren Erfolg v. a. auch vom Einsatz begeisterungsfähiger Einzelpersonen abhängig ist;

2) Nutzbarmachung von organisationsintern vorhandenen (familiären) Beziehungsstrukturen und persönlichen Netzwerken;

3) Akquise von externen, nicht-bekannten Personen durch das Angebot von Gegenleistungen. 
- Freiwilligenmobilisierung: Relevanz von Gegenleistung (MO) versus Motivierung von Eltern (RMO)

Das Angebot von Gegenleistungen als Mittel zur Mobilisierung von Engagierten hat für die säkularen Vereinigungen eine signifikant größere Relevanz. Für die religiösen Gemeinden ist hingegen die Motivierung von Eltern signifikant bedeutsamer.

Die anderen sechs zur Disposition gestellten Mobilisierungsaspekte ${ }^{138}$ scheinen somit (mit geringfügigen Varianzen) sowohl für die religiösen als auch säkularen Vereinigungen bedeutsam zu sein. Insofern erweist sich die im zweiten Teil der Hypothese $4\left(\mathrm{H}_{2}\right)$ theoretisch fundierte Annahme eines spezifischen Rekrutierungsprofils der religiösen MSO als theoretisch überspannt ${ }^{139}$. Wenngleich sich eine größere Bedeutung von Familientraditionen und regelmäßigen Veranstaltungen für die religiösen MSO abzeichnet, wird die Relevanz von bestehenden Beziehungsstrukturen statistisch lediglich in Bezug auf die Motivierung von Eltern, deren Kinder in den religiösen Vereinigungen an Aktivitäten teilnehmen, bestätigt. In diesem Zusammenhang wurde argumentiert, dass Familienstrukturen und kinderfreundliche Angebote, die auch informell und spontan ablaufen können, in den befragten religiösen MSO bedeutsamer sind. Zudem ist in diesem Zusammenhang die Multifunktionalität von religiösen MSO und insbesondere Moscheevereinen als viele Bedürfnisse befriedigende Dienstleistungszentren und lebendige Aufenthaltsorte für (Groß-)Familien ins Feld zu führen.

Dass der Erhalt von konkreten Gegenleistungen (z. B. Aufwandsentschädigungen, Weiterbildungen) für die Engagierten in nicht-religiösen Organisationen eine größere Rolle spielt, könnte auf deren professionellere Ausrichtung sowie eine geringere Relevanz von immateriellen Anreizen, wie dem Wunsch gute Taten zu vollbringen, wertekonform oder gottgefällig zu handeln, zurückzuführen sein.

Allerdings ist zu empfehlen, die Bedeutung von Mobilisierungsstrategien (für die beiden Organisationstypen) anhand einer größeren Analysestichprobe noch einmal erweitert zu überprüfen.

138 Durch Ansprache von Freunden und Bekannten; durch Ansprache von unbekannten Personen; durch motivierende und begeisternde Person(en); mit regelmäßigen Veranstaltungen; durch eine in den Familien gelebte Tradierung des Mitmachens; mit einer erfolgreichen Öffentlichkeitsarbeit.

${ }^{139} \mathbf{H 4}_{2}$ : Familientraditionen, regelmäßige Veranstaltungen sowie die persönliche Ansprache von Freunden/Bekannten im religiösen Feld sind besonders wirksame Faktoren für die Rekrutierung von Engagierten in religiösen MSO. 


\section{Einschätzung der Engagiertenmotive durch die Funktionsträger}

Der Auswertung und Interpretation von 13 vorgegebenen Motiven, von denen sich die Engagierten in den MSO am stärksten leiten lassen, wurde im Auswertungsteil viel Platz eingeräumt (7.1.2.4). Hier ist kritisch reflektiert worden, dass die Engagierten nicht selbst befragt und daher nicht die tatsächlichen Beweggründe der Engagierten auf der Individualebene erhoben wurden. Stattdessen erfolgte die Motivabfrage der Engagierten auf Basis der subjektiven Einschätzungen der Funktionsträger; dies beruht auf der theoretischen Prämisse, dass die Motive der Individuen auf die Gruppenebene ausgerichtet sind und diese damit unterstützen. Die Motivabfrage besitzt damit quasi eine Dummy-Funktion, eine testende Stellvertreterfunktion, da die menschlichen Motive für Engagement im Kontext organisationaler Rahmenbedingungen betrachtet werden, in die sie eingebettet sind (II. 4.3.3.2).

Anhand einer Explorativen Faktorenanalyse (EFA) lassen sich fünf Gruppen (Faktoren) inhaltlich zusammengehöriger Motive identifizieren:

1) Engagement aufgrund des Bedürfnisses nach sozialem Anschluss und lustorientierter Selbstverwirklichung;

2) Engagement aufgrund religiöser Überzeugungen;

3) Engagement aufgrund eines konkreten Eigennutzens und individueller Geltungsbedürfnisse;

4) Engagement aufgrund des Gefühls von Zusammengehörigkeit und Gleichgesinntheit in der Organisation;

5) Engagement aufgrund des Bedürfnisses nach Hilfeleistung, Mitgestaltung und Verbesserung vorgefundener (Lebens-)Situationen.

- Vergleich mit personenbezogenen Studienergebnissen: Kommensurabilitäten und Diskrepanzen

Es zeigt sich, dass die Funktionsträger von einer Vielfalt vorhandener Motive ausgehen: Neben lust- und geselligkeitsorientierten Beweggründen werden vorrangig Hilfeleistungen für andere und das Interesse an Weiterentwicklung und Verbesserung vorgefundener Gegebenheiten als relevante Triebfedern ausgemacht. Dieses entspricht Ergebnissen von auf der Individualebene durchgeführten Befragungen von türkeistämmigen Personen (Halm/Sauer 2005: 118 f.; Sauer 2011: 231 f.; s. auch Klie 2018: 444 f.). Zudem lassen sich aber auch Ähnlichkeiten mit für die deutsche Gesamtbevölkerung anhand des Freiwilligensurveys 2014 sowie einer Befragung des Allensbacher Instituts identifizierten Motivlagen feststellen (Müller et al. 2016: 418 f.; IfD Allensbach 2013: 27 ff.). Die Übereinstimmungen betreffen 
die Beweggründe, Spaß zu haben (FWS: $94 \%$; IfD: $95 \%$ ), mit anderen Menschen zusammenzukommen (FWS: $82 \%$ ), etwas bewegen (IfD: $83 \%$ ) oder die Gesellschaft mitgestalten (FWS: $81 \%$ ) zu wollen.

Diskrepanzen zeigen sich hingegen hinsichtlich des Erhalts von Anerkennung und Wertschätzung, was seitens der MSO-Vertreter mit 72 Prozent als wesentlich bedeutsamer eingestuft wurde (FWS: -40, IfD: -18 Prozentpunkte). Demgegenüber wird die Erwartung des Qualifikationserwerbs (für Beruf und Ausbildung) im Vergleich zu den Befunden des Freiwilligensurveys als für die in den MSO Engagierten (mit $36 \%$ ) weniger relevant gewichtet (FWS: + 16 Prozentpunkte); andererseits fällt die diesbezügliche Einschätzung wiederum höher aus als in der Allensbacher Studie (IfD: -17 Prozentpunkte) (s. für eine detailliertere Darlegung 7.1.2.4).

Während die Übereinstimmungen einerseits vermuten lassen, dass die Funktionsträger mit ihren Einschätzungen ganz richtig liegen, verweisen die Diskrepanzen andererseits auf die unterschiedlichen auf der Mikro- und Mesoebene angesiedelten Erhebungsarten: Im Organisationskontext wird der Befriedigung individueller Geltungsbedürfnisse durch gruppenbezogene Wertschätzung seitens der Funktionsträger eine größere Bedeutung beigemessen als dem persönlichen Eigennutzen der Engagierten.

- Religiöse Wertüberzeugungen (RMO) versus Profitorientierung und Optimierungsanliegen (MO)

Statistische Tests ermitteln eine größere Bedeutung der folgenden drei Motive in den religiösen Vereinigungen: ,religiöse Werte/Gebote umsetzen“, ,,anderen den Glauben nahebringen“ sowie „mit Gleichgesinnten zusammenzukommen“. In säkularen MSO besitzen hingegen die Erwartung von „Gegenleistungen“ und der Wunsch „Dinge verändern bzw. etwas bewegen zu wollen“ eine signifikant größere Relevanz.

Die jeweils höher eingeschätzten Motivbedeutungen spiegeln die unterschiedlichen Selbstverständnisse der beiden MSO-Typen und die in ihnen verschieden ausgerichteten Zweckorientierungen: Engagements aus religiösen, in der Gruppe geteilten Wertüberzeugungen stehen Engagements für einen konkreten Nutzen (Profit) und für Optimierung bestimmter Zustände im Sinne eines proaktiven Handelns gegenüber. 
- Kompatibilität von religiösen und nicht-religiösen Motivstrukturen.

Angesichts der Befunde lassen sich die Aussagen der Doppelhypothese $3\left(\mathrm{H} 3_{1}\right.$, $\mathrm{H}_{2}$ ), die sich auf das in religiösen und säkularen MSO jeweils unterschiedlich akzentuierte Engagement bezog ${ }^{140}$, nur teilweise bestätigen. Die Tatsache, dass die sehr eng gefasste Doppelhypothese als solche empirisch nicht bestätigt werden kann, legt nahe, dass gemeinschaftsbezogene religiöse Wertüberzeugungen sowie geselligkeits-, nutzen- und selbstbezogene Motive gut miteinander kompatibel und mit den (vielseitigen) Zielsetzungen, Interessen und Selbstverständnissen der MSO vereinbar sein können. Abgesehen von den im Rahmen dieser Organisationsbefragung hervorgetretenen Differenzen in Bezug auf religiöse Bedarfe, den Erhalt von Gegenleistungen und das Anliegen, Dinge zu verändern, scheinen sich religiöse und säkulare MSO hinsichtlich ihrer Verträglichkeit mit den theoretisch als einander ausschließend betrachteten Wertorientierungen bzw. Motiven gar nicht so sehr voneinander zu unterscheiden (s. 7.1.2.4 für eine detaillierte Einordnung der Ergebnisse). Diese theoretisch unerwartete Kommensurabilität zeigt, dass konträre Werthaltungen, nicht zuletzt aufgrund des dynamischen Wertewandels, miteinander gekoppelt sein können (vgl. auch Pickel 2018: 969 ff.; Pickel/Krüggeler 2001). Darüber hinaus werden in diesem Zusammenhang insbesondere für die in der Forschung im Interessenfokus stehenden Muslime unter Bedingungen kultureller Differenz verschiedene Wertorientierungskombinationen und Wertrichtungen geltend gemacht (Diehl/Koenig 2009; Nauck 2007; Ceylan 2012a; Nagel 2018a, b). Derlei Besonderheiten und Abweichungen werden in jüngeren personenbezogenen Studien für Muslime bestätigt, indem sie eine durchaus mögliche Kompatibilität von traditionell-konservativen und individualistisch-hedonistischen Wertvorstellungen anzeigen (Pollack/Müller 2013: 30; Sinnemann 2017: 29 f., 44). Diese in anderen Studien ermittelte Vereinbarkeit korrespondiert mit dem Ergebnis, dass persönliche Vergnügung für in religiösen MSO Engagierte als nicht weniger wichtig erachtet wird. Angesichts der Befundlage sollte den Aspekten von Konvivialität und Spaß, die sowohl in weltlichen als auch weltanschaulichen

${ }^{140} \mathbf{H 3}_{\mathbf{1}}$ : Aus Sicht der befragten Funktionsträger rangieren bei den Freiwilligen in religiösen MSO religiöse Wertüberzeugungen (anderen den Glauben nahebringen; religiöse Werte/Gebote umsetzen), gruppenbezogene Solidarität (mit Gleichgesinnten zusammenkommen; Solidarität mit der Organisation) und Hilfeleistung für andere vor anderen nutzen- und selbstbezogenen Motiven (Erwerb von Qualifikationen; Erhalt von Gegenleistung).

$\mathbf{H 3}_{2}$ : Die Motive der Freiwilligen, sich in nicht-religiösen MSO zu engagieren, bestehen nach Einschätzung der befragten Funktionsträger mit größerer Wahrscheinlichkeit aus selbstbezogenen Nutzenerwägungen (Erwerb von Qualifikationen; Erhalt von Gegenleistung), aus der Zuteilwerdung von Wertschätzung bzw. Anerkennung sowie aus der genussbetonten Bereicherung des eigenen Lebens (Freude haben). 
Organisationskontexten möglich und erwünscht sind, nicht nur - wie bislang geschehen - in Engagementuntersuchungen auf der Individualebene, sondern zukünftig auch in Anreizmodellen auf der Organisationsebene ein größerer Stellenwert eingeräumt werden (s. II. 4.4.1; vgl. Clark/Wilson 1961; Barnard 1970; Simon 1981/[1945]; March/Simon 1976/[1958]). Ebenfalls kritisch zu reflektieren ist, dass diese Gesichtspunkte auch in Organisationstypologien und Organisationstheorien marginalisiert und insbesondere in Hinblick auf religiöse bzw. weltanschauliche Organisationen weitestgehend ausgeblendet werden (vgl. u. a. Parsons 1960; Etzioni 1975; Blau/Scott 1962; Beckford 1975; Demerath et al. 1998; Sanders/Kianty 2006; Scott/Davis 2007; Beckford/Richardson 2007; Petzke/Tyrell 2012; Kühl 2015; Wilson 2020).

Das im Organisationskontext durchaus mögliche Zusammenspiel von religiösen und nicht-religiösen Motiven weist auch Jennifer Klöckner (2016) anhand von in Wohlfahrtsverbänden und MSO durchgeführten Engagierten-Befragungen nach. Klöckner zeigt auf, dass religiöse Motive in Kombination mit sozialen und kulturellen Aspekten genannt werden und schlussfolgert, dass sich in den religiösen Beweggründen von den in türkisch-islamischen Vereinen engagierten Personen zugleich das große Bedürfnis der Bewahrung der Herkunftslandlandkultur sowie der Kontaktpflege zur eigenen ethnischen Community manifestiere (ebd.: 434 ff.; vgl. auch Handy/Greenspan 2009: 957; Carabain/Bekkers 2010: 3). Auch hier spiegeln die Ergebnisse die multiplen Funktionen der MSO und insbesondere muslimischer Moscheevereine wider. Insgesamt ist also festzuhalten, dass das Engagement in religiösen Organisationen nicht vorschnell und ausschließlich auf religiöse Faktoren zurückgeführt werden sollte, sondern grundsätzlich von Motivbündeln und vielfältigen Anreizen auszugehen ist.

\section{Organisationsziele}

Auch die Zielsetzungen der MSO fallen vielschichtig aus. Anhand der Ergebnisse einer EFA lassen sie sich in drei Faktoren konzentrieren und wie folgt benennen:

1) Nachteilsausgleichende und gemeinwohlorientierte Vergemeinschaftung;

2) (Politische) Interessenvertretung und Teilhabe in Deutschland;

3) Herkunftsland- und Religionsbezogenheit. 
- Religiöse MSO als gesellige und soziale Orte

Den beiden Zielen, ein geselliges Beisammensein ${ }^{141}$ sowie die gemeinsame Glaubenspraxis zu ermöglichen, kommt in den religiösen Vereinigungen jeweils eine signifikant größere Bedeutung zu. Damit kann die Aussage unterstrichen werden, dass die religiösen MSO religiöse und soziale Orte zugleich sind, an denen das soziale Eingebundensein auch jenseits religiöser Zeremonien und weiterer religiöser Bedürfnisbefriedigungen eine bedeutende Rolle spielt; hingegen sind professionell ausgerichtete Angebote und Veranstaltungen - im Vergleich zu den säkularen Vereinigungen - weniger wichtig.

Die Ergebnisse korrespondieren mit den Motivbefunden, die ebenfalls verdeutlichen, dass religiöse MSO Plattformen sozialer Beziehungen sind, an denen nicht nur gemeinsam der religiösen Brauchtumspflege nachgegangen wird, sondern auch ein zwangloses Beisammensein für angenehmen Zeitvertreib und Vergnügung stattfinden kann.

Angesichts der Ergebnisse, die die Organisationsziele betreffen, kann allerdings nicht - wie in Hypothese 1 formuliert - unmittelbar davon ausgegangen werden, dass die Aspekte der Pflege der Herkunftskultur und des sozialen Miteinanders in den MSO tonangebend sind: Sie sind zwar bedeutsam, rangieren aber nicht an erster, sondern an vierter und fünfter Stelle (volle Zustimmung) im zweiten obersten Drittel hinter den auf Nachteilsausgleich von Gruppenmitgliedern und gesellschaftliche Mitgestaltung ausgerichteten Zielen (Abb. 7.7, 7.1.3). Hier zeigt sich, dass sich die theoretischen Annahmen in der Realität zwar als relevant erweisen, aber alleinig mit den beiden Items fokussiert - zu kurz greifen und als anachronistisch einzustufen sind.

- Höhere Relevanz von gesellschaftlicher Mitgestaltung und der Bewältigung sozialer Ausgrenzungserfahrungen für jüngere MSO

Denn im Generationenverlauf, mit fortdauerndem Aufenthalt, mit Geburt und Sozialisation in Deutschland wächst die Identifikation mit dem Heimat- und Lebensort, und Bedürfnisse, sich für allgemeine gesellschaftliche Interessen und Angelegenheiten einzusetzen, verstärken sich (BMFSFJ 2017: 200 f.). Dies schließt sowohl das selbstbewusste Einfordern chancengleicher Teilhabe als auch das Ausleben kultureller Prägungen sowie die Verarbeitung von im Herkunftskontext erlebter Diskriminierung und Ausgrenzung ein. Diese Annahmen werden durch die Ergebnisse gestützt, dass die zwei Ziele des Tätigseins für das Gemeinwohl in Deutschland und der Bewältigung von im Herkunftsland erfahrener Ausgrenzung und Vertreibung

${ }^{141}$ Einzustufen war das Ziel „Einen Ort der Geselligkeit und der Zusammenkunft schaffen“. 
für die jüngeren Vereinigungen von signifikant größerer Bedeutung und Aktualität sind als für ältere MSO; für letztere ist hingegen die gemeinsame Glaubenspraxis relevanter, was wiederum auf das höhere Alter der religiösen Vereinigungen zurückzuführen ist. Der bei jüngeren MSO stärker ausgeprägte Verarbeitungsbedarf von erfahrener Diskriminierung sollte mit nicht lange zurückliegenden Ereignissen zu erklären sein, die zum Verlassen des Heimatlandes geführt haben.

- „Geselligkeit“ als zentrales Scharnier zwischen binnen- und außenorientierter Integration

Anhand einer EFA konnte eine Querladung des Organisationsziels „Einen Ort der Geselligkeit und Zusammenkunft schaffen“ auf die Faktoren 1 und $3^{142}$ ermittelt werden. Dies lässt erkennen, dass Kontaktpflege und Geselligkeit innerhalb einer Organisation für beide Faktoren, die jeweils nach außen und nach innen gerichtete Organisationsziele umfassen, Relevanz besitzen. Demnach kann das soziale Miteinander innerhalb der Organisation zum einen als eine die Verbesserung der Lebenssituation und Unterstützung von benachteiligten Mitgliedern (Faktor 1) ermöglichende Voraussetzung eingestuft werden; denn das Miteinander fungiert als eine wichtige kohäsions- und konsolidierungsfördernde Größe. In diesem Zusammenhang ist zu erwägen, dass dieser Geselligkeitsaspekt - durch Gruppenkohäsion und binnenorientierte Aktivitäten (Faktor 3) - wiederum Erfahrungen von Eigenstärke, Autonomie und Kompetenz bewirken und damit zum anderen auch Kräfte freisetzen kann, die die Gruppe zur außenorientierten Beteiligung insbesondere in Minderheitensituationen animieren und befähigen (Faktor 1) (vgl. dazu auch Elwert 1982; Uslucan 2015, 2017a; II. 4.1). Insofern ist hier von einem interdependenten, integrativen Wirkungsgefüge mit Bezügen sowohl zum Herkunftsland als auch zur Aufnahmegesellschaft auszugehen und der Möglichkeit der Selbstorganisation bzw. dem Vergemeinschaftungsbedarf zwecks sozialer Beziehungen und Interaktionen (,Geselligkeit“) sind eine zentrale gruppenstabilisierende Scharnierfunktion beizumessen. Diese gruppenstabilisierende Scharnierfunktion kann „Zwischen Vergemeinschaftung und Vergesellschaftung“ (Pries 2010a: 37) vermitteln und die Gruppe sowohl nach innen als auch nach außen integrieren. Ihr kommt damit eine doppelte Integrationsfunktion zu: Soziabilität kann als ein Bindeglied zwischen gruppenbindendem und -überbrückendem Sozialkapital fungieren. Angesichts dieser Schlussfolgerungen spricht die Querladung für die Bekräftigung einer positiv wirksamen Binnenintegration und - auf den zweiten Blick - damit für die

${ }^{142}$ Faktor 1: „Nachteilsausgleichende und gemeinwohlorientierte Vergemeinschaftung“; Faktor 3: „Herkunftsland- und Religionsbezogenheit“ (s. o.). 
Bestätigung des sich auf die Sozialität als zentrales Organisationsziel beziehenden Teils der Hypothese 1 . $^{143}$

In diesem Kontext ist noch einmal die Annahme von Georg Elwert (1982) anzuführen: Ihm zufolge sind die mit der Binnenintegration einhergehende Markierung und Bewahrung einer eigenständigen kulturellen Identität durchaus mit Kontaktaufnahmen zur Mehrheitsgesellschaft (der „Außenwelt“) und Prozessen der Akkulturation kompatibel (ebd.: 726). Das bedeutet, dass eine starke Integration von Einwanderern innerhalb eigenethnischer Sozialstrukturen , unter bestimmten Bedingungen ein positiver Faktor für ihre Integration in eine aufnehmende Gesellschaft" (Elwert 1982: 718; Herv.: d. Verf.) sein kann (II. 4.1). Das eigenkulturelle Sozialkapital sollte daher als ein wichtiger Faktor gesellschaftlicher Integration nicht relativiert werden. Jedoch ist eine wichtige Bedingung für die Realisierung dieser integrationsermöglichenden Scharnierfunktion, dass sich religiöse oder ethnische Vereinigungen - jenseits kollektiver Identitäten und Loyalitäten - der Existenz und Bedeutsamkeit von gesellschaftspolitischen Belangen als einen weiteren Referenzrahmen bewusst sind und dass sie an integrationsrelevanten Aktivitäten und Bereichen teilnehmen. Dies impliziert die Durchführung gemeinsamer gruppenübergreifender Aktivitäten, die auf die Verwirklichung von gesellschaftlich als wichtig und nützlich erachteten Zielen gerichtet sind. Um diese Interaktion und Teilhabe der Gruppenmitglieder zu gewährleisten, müssen wiederum ermöglichende Bedingungen und Handlungsfähigkeiten vorhanden sein. Dazu gehören v. a. die Offenheit anderer Gesellschaftsmitglieder und Institutionen, aber auch die Befähigung und Bereitschaft, sich auf die in der aufnehmenden Gesellschaft geltenden Verhältnisse einzulassen.

- Organisationsziele: Dominant auf Deutschland ausgerichtet und unpolitisch

Insgesamt sind die Ziele der MSO dominant auf Deutschland und die Verbesserung der Situation von Landsleuten bzw. Menschen mit einer Zuwanderungsgeschichte ausgerichtet. Zugleich beziehen sie sich auf die kollektive, soziale Ebene des gesellschaftlichen Umfelds als eine wichtige Voraussetzung für ein gelingendes Zusammenleben. In Bezug auf die Relevanz und Mitgestaltung von (partei-) politischen Verhältnissen im Herkunftsland oder in Deutschland zeigen sich die im Rahmen der vorliegenden Studie befragten MSO insgesamt eher unpolitisch.

Werden die als wichtig erachteten Ziele ernst genommen und umgesetzt, dann sind die MSO potenziell imstande, eine bedeutende Brückenfunktion zu

143 H1: Verglichen mit anderen Zwecken stellen die Aufrechterhaltung der Herkunftskultur und die Schaffung eines Ortes der Sozialität sowohl für nicht-religiöse als auch religiöse MSO besonders wichtige Organisationsziele dar. 
übernehmen, indem Belange sowohl der Minderheits- als auch der Mehrheitsgesellschaft seitens der Gruppenmitglieder berücksichtigt werden und eine Vermittlung zwischen unterschiedlichen Ausgangspositionen und Bedürfnislagen stattfinden kann. In ihrer diesbezüglichen Relevanz in Betracht zu ziehen sind auch positive Rückkopplungsprozesse: Dominant auf die Aufnahmegesellschaft bezogene Ziele, die sich in entsprechenden Aktivitäten widerspiegeln und die durch aufnahmegesellschaftliche Akteure bekräftigt werden, können zur Konsolidierung der Organisationen beitragen und dadurch wiederum die Erweiterung ihrer sozialen Wirkungsräume forcieren.

\section{Selbstverständnisse der MSO}

- Netzwerke und Förderorganisationen (MO) versus Gemeinschaften Gleichgesinnter (RMO)

In der Mehrzahl werden die MSO als Brückenbauer und Kulturdolmetscher (85\%) sowie Integrationspromotoren $(82 \%)$ betrachtet. Damit werden in der politischen Debatte für migrantische Vereinigungen stark gemachte Funktionen durch deren Selbstdefinitionen quasi bestätigt.

Während sich die religiösen Vereinigungen signifikant häufiger als Religionsgemeinschaften und Gemeinschaften Gleichgesinnter verstehen, ist es für die säkularen MSO signifikant bedeutsamer, Netzwerke und Förderorganisationen zu sein. Damit unterstreichen die religiösen MSO die Relevanz von Verbundenheit und geteilten Werten innerhalb der eigenen Gemeinschaft; die säkularen Vereinigungen markieren hingegen ihr Interesse an Austausch und Verbindung mit anderen Akteuren sowie ihr Anliegen, Entwicklung und Vorankommen von Prozessen aktiv unterstützen zu wollen.

Davon unabhängig spiegeln die Ergebnisse die mehrfach verorteten Kollektividentitäten der Vereinigungen. Sowohl bei den religiösen als auch bei den säkularen Organisationen geht die Besinnung auf die eigene Gruppe mit gesellschaftsrelevanten Außenorientierungen einher, die auch selbstverpflichtende Momente implizieren, wie etwa eine „fördernde“ oder ,dienstleistende“ Organisation zu sein.

\section{Das soziale Miteinander in den Organisationen}

- Geringe Konfliktdichte und große Bedeutung demokratischer Strukturen sowie gleicher Geschlechterbeteiligung

Es zeigt sich für die religiösen Vereinigungen eine signifikant größere Bedeutung der gruppenstabilisierenden Wirkung des geteilten Glaubens sowie der gemeinsam praktizierten Rituale bzw. regelmäßigen Zusammenkünfte. 
In nicht signifikantem Ausmaß werden in den religiösen MSO zwischen den jungen und den älteren Mitgliedern häufiger Konflikte registriert. Dieser Befund ist interessant und erwähnenswert, da er aus migrations- und religionssoziologischer Perspektive mit anders gelagerten religiösen und sozialen Orientierungen und Bedürfnislagen der jüngeren, in Deutschland sozialisierten Generationen zu erklären wäre (I. 2.2; vgl. Schiffauer 2010; Ceylan 2012a; Antes/Ceylan 2017; Nagel 2015a, 2018a, b). Demnach könnten für die jüngeren Generationen mit der Frequentierung von religiösen Vereinigungen bestimmte religiöse Vorstellungen, Erwartungshaltungen und Relevanzkriterien einhergehen, die sich von denjenigen älterer Gemeindemitglieder unterscheiden. Ein solch zu erwartendes Phänomen tritt im Rahmen dieser Studie jedoch nicht als für die Situation in religiösen MSO symptomatisch hervor.

In Hinblick auf die hohen Zustimmungsraten zur Wichtigkeit von demokratischen Strukturen und gleichberechtigter Geschlechterbeteiligung in den Organisationen (96\%) wurde einerseits argumentiert, dass diese Aspekte wesentliche Charakteristika von freiwilligen Vereinigungen spiegeln. Denn in diesen besteht grundsätzlich eine Abhängigkeit von Mitgliedern und Engagierten, deren Interessen im Organisationsgeschehen folglich adäquat zu berücksichtigen und einzubinden sind (II. 4.3.4). Andererseits wurde zu bedenken gegeben, dass die Vereinigungen eine intakte Organisationkultur und ein progressives Image vermitteln wollen, um beispielsweise verbreiteten Ansichten zur Unterdrückung der Frau im Islam oder in anderen Kulturen entgegenzuwirken.

Darüber hinaus scheint die Aufteilung von Tätigkeiten zwischen den Hauptamtlichen und Engagierten überwiegend nicht in Konkurrenzsituationen zu münden (91\%) und ohne größere Probleme zu verlaufen.

\section{Tätigkeitsbereiche der MSO}

- (Sozial-)Pädagogische und sozialarbeiterische Dienstleistungen dominieren

Die Ergebnisse zu den Aktivitätsschwerpunkten spiegeln ein breites Spektrum und eine vielfältige Mischung der Aufgaben und Funktionen, die die MSO wahrnehmen. Deutlich wird die Präferenz, unpolitisch sein bzw. politisch nicht mitgestalten zu wollen; sie korrespondiert mit der geringen Zustimmung zum Organisationsziel, parteipolitische Positionen in Deutschland unterstützen zu wollen.

Die Aktivitäten lassen sich auf Basis der Durchführung einer EFA bündeln und folgenden vier Faktoren zuordnen:

1) (Sozial-)Pädagogische und sozialarbeiterische Dienstleistungen, die Stadtteiloder Gemeinwesenarbeit umfassen; 
2) Aktivitäten der Kulturpflege sowie Freizeit/Geselligkeit;

3) Unterstützungsleistungen, Problembewältigung und Interessenvertretung in der Gemeinschaft sowie in öffentlichen und internationalen Rollen;

4) Religiöse Aktivitäten.

- Die religiösen MSO sind hinsichtlich der Anzahl der Tätigkeitsbereiche aktiver Insgesamt werden in vielen Bereichen Tätigkeiten und Dienstleistungen übernommen. Eine Aufsummierung zeigt, dass über ein Drittel der MSO (35\%) schwerpunktmäßig in 10 bis 13 und ein gutes weiteres Viertel (26\%) in 14 Bereichen aktiv sind. Die religiösen Vereinigungen sind mit Blick auf die Anzahl der Bereiche signifikant aktiver. Die Ergebnisse spiegeln die in der Forschung insbesondere für islamische Vereinigungen konstatierte Multifunktionalität, aber insgesamt auch die beachtenswerte Leitungsfähigkeit der über disparate Ressourcenausstattungen verfügenden MSO.

- Bei vielen Gemeinsamkeiten: Religiöses Tätigkeitsprofil in religiösen und heterogenere Aktivitätsfelder in säkularen MSO

Für die religiösen MSO kann jeweils eine signifikant größere Bedeutsamkeit der drei Tätigkeiten religiöse Brauchtumspflege, religiöse Bildung/Erziehung sowie interreligiöser Dialog ermittelt werden. Zudem spielt in den religiösen MSO auch die Arbeit mit Senioren eine größere Rolle. In den säkularen MSO besitzen hingegen die drei Tätigkeitsbereiche Kultur allgemein, Bildung/Erziehung sowie Arbeitsmarktzugang eine jeweils signifikant größere Bedeutung.

Neben diesen Unterschieden zeigen sich in den Tätigkeitsbereichen der religiösen und nicht-religiösen MSO v. a. auch Gemeinsamkeiten: Für die Arbeit mit Kindern bzw. Jugendlichen sowie mit Mädchen bzw. Frauen, für die Deutschförderung, Stadtteilarbeit, Flüchtlingshilfe, Internationale Arbeit, die Pflege der Herkunftskultur, Freizeit \& Geselligkeit sowie Sport \& Bewegung sind keine signifikanten Differenzen auszumachen. Dies ist ein Beleg für das vielfältige Nebeneinander zivilgesellschaftlicher Performanzen - unabhängig von spezifisch verorteten kollektiven Bindungen. Zudem zeigt sich damit auch, dass die beiden konsequent verglichenen MSO-Typen recht konform Tätigkeiten der Sozialen Arbeit (Wohlfahrt) nachgehen, wobei dies mit Blick auf die Seniorenarbeit für die religiösen MSO stärker zutrifft. Dies verweist auf den angesichts des demografischen Wandels wachsenden Stellenwert der Bereitstellung sozialer Dienstleistungen insbesondere auch für ältere Menschen in muslimischen und alevitischen Gemeinden in Deutschland (Halm/Sauer 2015: 105). Im Vergleich dazu fallen die für säkulare MSO signifikant bedeutsameren anderen drei Sparten (Kultur; Bildung/Erziehung; Arbeitsmarkt) 
hinsichtlich kulturell-sozialer sowie strukturell-integrativer Implikationen als solche durchmischter und uneinheitlicher aus.

- Religiöses Verhalten auch in nicht-religiösen MSO

Dass religiöse Vereinigungen dominant glaubensbasierte Aktivitäten ausüben und sich religiöser gerieren als die säkularen, ist kein überraschender Befund. Dies bedeutet jedoch nicht, dass religiöse Orientierungen und Praktiken in formal keiner Glaubensgemeinschaft zuzuordnenden Vereinigungen grundsätzlich keine Rolle spielen. Auch dies ist plausibel, denn religiöses Verhalten setzt keine offizielle Bekundung von religiösen Zugehörigkeiten voraus. Ähnlich wie bei der Mitgliederstruktur oder dem sozialen Miteinander in den MSO (7.1.2.1; 7.1.3) wird hier deutlich, dass die per Selbstdefinition vorgenommene Einteilung in religiöse und säkulare MSO eine hilfreiche Kategorisierung darstellt, die jedoch nicht zur pauschalen Annahme verleiten sollte, dass sich in entsprechend klassifizierten Vereinigungen jeweils konsequent (nicht-)religiös verhalten wird. So kann religiösen Orientierungen und Handlungen in den als „nicht-religiös“ kategorisierten MSO potenziell eine größere Bedeutung zukommen als dies das Labeling der Organisation vermuten ließe ( Spotlight: Die religiöse Performanz der MSO).

\section{Aktivitäten in der Flüchtlingshilfe}

Fast die Hälfte der MSO (47 \%) übernimmt Aufgaben in der Flüchtlingshilfe. Auf den obersten Rängen stehen die Vermittlung und Erklärung rechtlicher und kultureller Regeln in Deutschland (91\%), die Unterstützung bei Behördengängen $(88 \%)$, Dolmetschertätigkeiten ( $86 \%$ ), Hilfestellungen beim Deutschlernen (76\%), eine gemeinsame Freizeitgestaltung ( $72 \%$ ) sowie Unterstützungen bei der Wohnungssuche $(70 \%)$.

- Gemeindenahe Unterstützungsleistungen in religiösen Vereinigungen versus höhere Affinität zu administrativ-rechtlichen Angelegenheiten in säkularen MSO

Statistische Tests tragen in Bezug auf die Flüchtlingshilfe zur Profilbildung der beiden Organisationstypen bei: Neben der religiösen Betreuung in den religiösen MSO sind dort auch die vier Aktivitäten Bereitstellung von Essen und Trinken, Spendenverwaltung, Seelsorge sowie Fahrdienste von größerer Bedeutung. In den säkularen Vereinigungen werden geflüchtete Menschen hingegen häufiger bei Behördengängen unterstützt. Diese Befunde sprechen dafür, dass insbesondere religiöse Vereinigungen als Orte und Anlaufstellen fungieren, an denen eine adäquate religiöse bzw. seelsorgerische Betreuung von religiös orientierten geflüchteten Menschen 
stattfinden kann. Zudem ist zu vermuten, dass die häufigere Übernahme von primär nicht religiös ausgerichteten Tätigkeiten - wie die Verpflegung von Geflüchteten, die Verwaltung von Spenden sowie Fahrdienste - aufgrund regelmäßiger Interaktionen und gekoppelt an gut organisierte, intakte Infrastrukturen der Gemeinden bzw. im Rahmen vorhandener Angebote und Dienstleistungen erfolgt. Anzunehmen ist, dass die vorhandenen Infrastrukturen, gemeinsame Herkunftsbezüge, Sprachen und Glaubenszugehörigkeiten sowie geteilte Erfahrungen und Bedürfnisse einen guten Ausgangspunkt und Nährboden für Solidarität und den Aufbau von Vertrauen zu vulnerablen Gruppen geflüchteter Menschen darstellen können (vgl. auch Nagel/El-Menouar 2017: 27, 45; Friedrichs et al. 2020: 31).

Das Phänomen, dass in säkularen Vereinigungen häufiger eine Unterstützung bei Behördengängen erfolgt, sollte auf eine höhere Affinität zu administrativen und juristischen Angelegenheiten bzw. strukturellen Integrationsmaßnahmen sowie einen besseren Draht der säkularen MSO zu amtlichen Dienststellen und Verwaltungsorganen zurückzuführen sein.

\section{Veränderungen für die MSO seit der Fluchtdynamik 2015}

Für 63 Prozent der in der Flüchtlingshilfe aktiven MSO trifft die Aussage zu, dass ihre Organisation zur Anlaufstelle für Geflüchtete geworden ist. 53 Prozent der MSO bestätigen, dass ihre Arbeit seitens der Stadt und Politik eine höhere Aufmerksamkeit und Wertschätzung erfahren. Für 65 Prozent hat sich die Zusammenarbeit mit städtischen Ämtern und Behörden intensiviert. Von politischen Fördermaßnahmen profitierten zum Zeitpunkt der Befragung 37 Prozent der in der Flüchtlingshilfe aktiven MSO. Für religiöse und nicht-religiöse MSO zeigen sich Unterschiede, die eine ungleiche Behandlung andeuten.

- Größere stadtpolitische Anerkennung von und Zusammenarbeit mit säkularen MSO

Seit der Fluchtdynamik nehmen die säkularen MSO signifikant häufiger eine gestiegene Aufmerksamkeit und Wertschätzung seitens der Kommunen und Politik wahr; für sie hat sich auch die Zusammenarbeit mit städtischen Ämtern und Behörden in einem signifikant größeren Ausmaß intensiviert. Zudem haben die säkularen MSO häufiger von politischen Fördermaßnahmen in der Flüchtlingshilfe profitiert, allerdings nicht in signifikanter Größenordnung. Die Ergebnisse korrespondieren mit der Feststellung einer für religiöse MSO niedriger ausfallenden finanziellen 
Förderung durch die Städte (und das Land NRW) und lassen sich im Kontext der auf kommunaler Ebene in NRW bestehenden Richtlinien für die Förderung von und Zusammenarbeit mit MSO deuten: Unabhängig von religiösen und kulturellen Aktivitäten ist für die Möglichkeit einer Förderung ausschlaggebend, dass seitens der MSO Integrationsarbeit geleistet wird (Klie 2020; vgl. auch Friedrichs et al. 2020: 64 ff.). Da bei religiösen MSO seit der sogenannten „Flüchtlingskrise“ 2015 ein erhöhter Zulauf von Geflüchteten registriert worden ist, kam es zu einer stärkeren Projektförderung von Moscheegemeinden in der Flüchtlingshilfe. Allerdings werden in einigen MSO auch Rückzugstendenzen und in den türkischen Moscheegemeinden eine starke (politische) Beeinflussung der Mitglieder durch das Heimatland registriert (ebd.; Güvercin 2019). Zudem haben die politischen Entwicklungen in der Türkei, die daraus resultierende Verschlechterung der bilateralen Beziehungen zu Deutschland und die Beobachtung einer starken, nicht geduldeten Einflussnahme zu Verunsicherungen und einem Rückgang der städtischen Zusammenarbeit mit und Anerkennung von islamischen MSO geführt.

Neben dieser Erklärungsfolie wurde im Rahmen der Ergebnisdiskussion zudem darauf verwiesen, dass die säkularen MSO eine dezidierte Ausrichtung auf kommunalpolitisch etablierte Tätigkeitsfelder, die die Unterstützung und Integration von Menschen mit Migrations- und Fluchthintergrund betreffen, aufweisen dürften und dass sich ihre Tätigkeitsbereiche und Interessenlagen daher stärker mit denjenigen von anderen in der Integrationsarbeit offiziell eingebundenen Akteuren überschneiden könnten. Die unterschiedliche Ausgangslage für säkulare und religiöse MSO muss folglich nicht zwangsläufig auf Reglementierungsbedarfe und Sanktionsmechanismen zurückzuführen sein, sondern sie kann sich ggf. auch einfach ergeben und entwickelt haben, da nicht-religiöse MSO und städtische Einrichtungen stärker und gemeinsam in der Integrationsarbeit tätig sind.

Nicht zuletzt kann jedoch auch das im Folgenden noch darzulegende insgesamt deutlich weniger intensive Vernetzungsverhalten der religiösen MSO als Erklärung für die ungleichen Verhältnisse herangezogen werden.

\section{Zielgruppen der Aktivitäten}

- 83 Prozent der MSO für alle Zielgruppen offen

Mit einer deutlichen Überzahl gaben 83 Prozent der MSO unter der Bedingung nur einer zulässigen Antwort (Einfachnennung) an, dass ihre Aktivitäten und Angebote für alle offen sind. Bei 7 Prozent der MSO richten sich die Angebote und Dienstleistungen der MSO nur an Mitglieder und bei 4 Prozent und ausschließlich bei religiösen MSO (14\%) sind die Aktivitäten nur für Personen mit den gleichen religiösen Überzeugungen vorgesehen. 


\section{Aktivitätsradien der MSO}

- Insgesamt vielfältige und große Aktivitätsradien der MSO

In Hinblick auf die Aktivitätsradien der MSO konnte festgestellt werden, dass die MSO mit rund 17 Prozent in der Mehrzahl ausschließlich in der Stadt aktiv sind. ${ }^{144}$ Die säkularen und religiösen MSO weisen sehr ähnliche Aktivitätsradien auf; die religiösen sind etwas häufiger im Stadtteil und in der Stadt aktiv. Des Weiteren sind viele MSO in unterschiedlichen Kombinationen im Stadtteil, in der Region, in NRW, Deutschland, Europa oder international aktiv. Hier sind vereinzelt große „Ebenensprünge“ zu registrieren, d. h. Tätigkeiten im Nahraum, etwa im Stadtteil oder in der Stadt, werden auch unter Auslassung der Ebenen „Region“ oder „NRW“, von Tätigkeiten in Deutschland, Europa und/oder im internationalen Raum flankiert.

- Große Bedeutung von Beziehungen im nahen, fernen und ,intermediären ‘ Raum

Es wurde argumentiert, dass die Existenz von Beziehungen im nahen, fernen und intermediären Raum als Formen von Solidarität oder Sozialkapital betrachtet werden können, die auf einem Kontinuum zwischen den extremen Endpolen des sozialen nachbarschaftlichen Nahraums und des transnationalen Fernraums zu denken sind (7.1.5). Die einzelnen und unterschiedlichen (Misch-)Formen von sich auf verschiedenen und mehreren Ebenen abspielenden Beziehungen und Interaktionen sollten insbesondere für Selbstorganisationen von Menschen mit Zuwanderungsgeschichte bedeutsam sein. Es könnte erkenntnisreich sein, diese Beziehungsebenen und ihre (Aus-)Wirkungen zukünftig stärker im Kontext der transnationalen Ausrichtung von MSO und deren Verbindungen zu Diaspora-Standorten zu untersuchen (s. dazu auch Pries/Sezgin 2010a; Pries 2001; Nagel 2013a; Lauser/Weißköppel 2008).

\section{Kooperationspartnertypen}

- Städtische Einrichtungen, andere MSO und ortsansässige Vereine als häufigste Kooperationspartner

Gut drei Viertel der MSO (76\%) arbeiteten zum Zeitpunkt der Befragung mit anderen Organisationen zusammen oder haben dieses in den letzten fünf Jahren getan. Unter den kooperierenden MSO sind religiöse und nicht-religiöse Vereinigungen zu fast gleichen Anteilen vertreten. Städtische Einrichtungen (68\%), MSO (67\%) und ortsansässige Vereine (61 \%) stellen die häufigsten Kooperationspartner der befragten MSO dar. Damit sind für die MSO in NRW im Vergleich zu den Befunden

144 Vorgegeben waren sieben Wirkungsbereiche: „im Stadtteil“; ,in der Stadt“; ,in der Region (Kreis/Bezirk)“; ,,in Nordrhein-Westfalen“; ,,in ganz Deutschland“; ,,in ganz Europa“; „International“. 
des ZiviZ-Surveys insgesamt deutlich stärker ausgeprägte kooperative Verhältnisse zu den Kommunen festzustellen. ${ }^{145}$ Zugleich zeigen sich viele Ähnlichkeiten mit den Ergebnissen der Studie des SVR-Forschungsbereiches, der zufolge 65 Prozent der befragten MSO mit Stadtverwaltungen, 64 Prozent mit anderen gemeinnützigen Vereinen und 61 Prozent mit anderen MSO ,partnerschaftlich“ zusammenarbeiten. Währen in der dieser Arbeit zugrunde liegenden Untersuchung rund 39 Prozent der MSO mit Wohlfahrtsverbänden kooperieren, sind es in der SVR-Erhebung 30 Prozent (Friedrichs et al. 2020: 59).

- Die säkularen MSO haben deutlich vielfältigere Kooperationspartner

Es zeigt sich, dass die säkularen MSO mit einer signifikant größeren Anzahl von Organisationstypen zusammenarbeiten: Sie kooperieren signifikant häufiger mit Organisationen oder Ämtern in der eigenen Stadt und mit staatlichen Organisationen (z. B. Bundes- oder Landesministerien), mit Universitäten und Wohlfahrtsverbänden wie auch Stiftungen, Integrationsagenturen, Freiwilligenagenturen (bzw. Mehrgenerationenhäusern) und privatwirtschaftlichen Unternehmen. Für religiöse MSO stellen hingegen Kirchen bedeutsame Kooperationspartner dar. Für die beiden MSOTypen ist somit von einem unterschiedlichen Kooperationsverhalten auszugehen, was darauf hindeutet, dass unter den beteiligten Akteuren jeweils spezifische (professionelle) Partner präferiert oder begünstigt werden. Darüber hinaus zeigt sich eine Variationsbreite von Kooperationspartner-Kombinationen, die keine dominanten Häufigkeiten erkennen lassen.

Das für die säkularen MSO festzustellende breitere Spektrum an Kooperationspartnern kann auf die Verfügbarkeit größerer Netzwerke und die Präferenz oder Bevorteilung bestimmter Partnerorganisationen zurückzuführen sein. Zudem kann die strategische Verfolgung spezifischer Anliegen eine Rolle spielen. In diesem Zusammenhang sind auch förderpolitische Richtlinien relevant, die letztlich die nicht-religiösen MSO begünstigen, da sie eine größere Nähe zu integrationspolitischen Aktivitäten bzw. eine dezidierte Ausrichtung auf solche aufweisen dürften.

In Hinblick auf die religiösen MSO wurde eine größere Selbstgenügsamkeit oder „habituelle Zurückgenommenheit“" (Nagel 2015b: 29) thematisiert, die der zweckgerichteten Erlangung spezifischer Vorteile oder zusätzlicher (finanzieller) Nutzen entgegenstehen könnte. Dieser Deutungshorizont ist kompatibel mit dem primären Selbstverständnis der religiösen MSO, Religionsgemeinschaften und Gemeinschaften Gleichgesinnter zu sein, während sich die säkularen Vereinigungen in einem signifikant stärkeren Ausmaß als Förderorganisationen und Netzwerke verstehen.

${ }^{145}$ Den Daten des ZiviZ-Surveys zufolge sehen sich 22 Prozent der MSO in einem kooperativen Verhältnis zu Kommunen, 21 Prozent stehen in einem Auftragsverhältnis zu einer Kommune (Priemer 2017: 2; I. 2.1). 
Darüber hinaus könnte es sich auch so verhalten, dass seitens der religiösen MSO keine passenden Kooperationspartner gefunden wurden.

An dieser Stelle ist jedoch auch noch einmal grundsätzlich anzumerken, dass Kooperationen die Bereitschaft und Offenheit aller beteiligten Akteure voraussetzen. Ein Nicht- oder Wenig-Kooperieren kann freilich mehrere Ursachen haben (II. 3.2; II. 4.3.5; II. 4.5).

\section{Ziele der Kooperationen mit anderen Organisationen}

- Breitere und taktischere Ausrichtung der Kooperationsarbeit unter den säkularen MSO

Für sechs Kooperationsziele zeigte sich jeweils eine statistisch signifikante gröBere Bedeutung für die säkularen Vereinigungen. Bei diesen handelt es sich um die Verbesserung der Bekanntheit und Vernetzung der Organisation, den Erhalt von finanziellen Mitteln, den Informationsgewinn, die Etablierung einer langfristigen Zusammenarbeit, die Verbesserung von Kontakten zu Zielgruppen sowie die Hervorhebung der Nützlichkeit der Angebote der MSO. Dies verweist auf eine im Vergleich zu den religiösen MSO grundsätzlich breitere und taktischere Ausrichtung der Kooperationsarbeit der säkularen Vereinigungen. Dafür spricht auch die größere Vielfalt ihrer Kooperationspartner.

- Die zentrale Bedeutung öffentlicher Bekanntheit und Vernetzung

Im Rahmen der Darlegung der theoretischen Annahmen des soziologischen Neoinstitutionalismus (NI) wurde mit Blick auf zentrale Kooperationsziele die Hypothese 6 formuliert. Mit dieser wurden als für die MSO besonders wichtige Kooperationsziele 1.) die Hervorhebung von Umfang und Nützlichkeit ihrer Aktivitäten, 2.) die Steigerung der Bekanntheit und Vernetzung, 3.) der Abbau von wahrgenommenen Vorbehalten sowie 4.) die Verbesserung der Kontakte zu Politikern unterstellt. Die Hintergrundfolie bildet der im NI postulierte Leitgedanke, dass Organisationen nach der Erlangung von Legitimität streben, da ihnen nur mit einem Legitimitätsstatus langfristig Anerkennung, Wertschätzung und Unterstützung zuteilwerden können.

Auf den ersten Blick scheinen die Untersuchungsergebnisse die Hypothese zu falsifizieren, da die als besonders bedeutsam eingestuften Zwecke unter allen Kooperationszielen die mittleren bzw. unteren Ränge einnehmen (Abb. 7.7, 7.1.3).

Als aufschlussreich erwies sich die Durchführung einer EFA, mit der zwei Faktoren identifiziert und benannt werden konnten: 
1) Wettbewerbsorientierte Besserstellung und Legitimitätsgewinn der MSO;

2) Qualifizierung und Ausweitung der Arbeitsbereiche.

Angesichts der Faktorladungsmatrix wurde einerseits argumentiert, dass die mit vier Kooperationszielen in der Hypothese 6 verknüpften theoretischen Annahmen zu eng gefasst wurden. Andererseits ist die Querladung des Items „Verbesserung von Bekanntheit und Vernetzung" auf beide Faktoren als ein Indiz für die Bestätigung der Annahmen des NI zu deuten. Denn dieses Ziel kann als eine wesentliche Bedingung für die praktische Verwirklichung der jeweils auf die Faktoren 1 und 2 ladenden Ziele (Items) betrachtet werden: Es kann angenommen werden, dass öffentliches Bekanntsein (auch im Sinne von Popularität) und soziale Kontakte für kooperationswillige MSO grundsätzlich von zentraler Bedeutung sind. Insofern sind Kooperationsziele hinsichtlich des Gewinns von Anerkennung und Legitimität nicht trennscharf zu fassen, sondern in fließenden Übergängen miteinander verquickt.

- Größere Bedeutung von Finanzierungs- und Planungssicherheit für jüngere MSO

Des Weiteren zeigte sich, dass hinsichtlich der Bedeutsamkeit der Kooperationsziele und der Legitimitätsrelevanz das Alter der MSO von Belang ist: Den drei Zielen der Erschließung von zusätzlichen finanziellen Mitteln, des Aufbaus einer langfristigen Zusammenarbeit sowie der Verbesserung der Bekanntheit und Vernetzung der Organisation kommt in jüngeren MSO jeweils eine größere Bedeutung zu. Darin spiegelt sich der für die jüngeren Vereinigungen relevante höhere Bedarf an einer Finanzierungs- und Planungssicherheit sowie eine stärker auf Nachhaltigkeit ausgerichtete Zukunftsplanung.

Die für jüngere MSO signifikant größere Bedeutung des querladenden Kooperationsziels („Verbesserung von Bekanntheit/Vernetzung"), die sich mit den beiden für sie ebenfalls relevanteren, den Faktoren 1 und 2 zuzuordnenden Zwecken kombiniert, untermauert paradigmatisch die oben akzentuierte Interpretation einer die anderen Kooperationszwecke ermöglichenden ,Hebelfunktion “ des querladenden Kooperationsziels zwischen unterschiedlich ausgerichteten Zwecken.

Angesichts der Ergebnisinterpretation ist einerseits zu schlussfolgern, dass der $\mathrm{NI}$ weiter zu denken ist, da unterschiedlich ausgerichtete Kooperationsziele miteinander verknüpft sind und der NI - zumindest in quantitativen Befragungen - über Kooperationsziele schwer einzuhegen und zu operationalisieren ist. Die hiesigen Befunde sprechen dafür, dass der Gewinn von Legitimität immer in irgendeiner Form relevant ist. Andererseits könnte sich angesichts der Bündelung mehrerer Kooperationszwecke für den Versuch einer erkenntniserweiternden Prüfung der Theorie des 
NI ein größerer Stichprobenumfang sowie eine breitere, kleinteiligere inhaltliche Operationalisierung der Kooperationsziele als opportun erweisen.

\section{Wahrgenommene Schwierigkeiten bei Kooperationen}

- Größere Konkurrenzsituation und Abhängigkeitsverhältnisse für die säkularen MSO

Rund 50 Prozent der kooperierenden MSO gaben an, innerhalb der Zusammenarbeit mit anderen Organisationen mit Schwierigkeiten konfrontiert zu sein. Es zeigt sich, dass in erster Linie ein zu hoher, komplizierter Verwaltungsaufwand (62 \%), unterschiedliche Auffassungen über die gemeinsame Projektumsetzung (56\%) sowie eine geringe Offenheit und Vorurteile gegenüber der eigenen Organisation (54\%) als Hemmnisse wahrgenommen werden.

Konkurrenzsituationen um Geld, Ideen oder Zielgruppen (39\%) sowie bestehende inhaltliche und finanzielle Beeinflussungen und Abhängigkeiten (36\%) werden signifikant häufiger von nicht-religiösen Vereinigungen als Kooperationsbarrieren wahrgenommen. Diese Ergebnisse können auf die größere Anzahl und Vielfalt der seitens nicht-religiöser Vereinigungen gewählten Kooperationspartner zurückzuführen sein, da diese auf unterschiedlichen gesellschaftspolitischen und administrativen Ebenen operieren und die Zusammenarbeit daher unter spezifischen Umständen wettbewerbsorientierter und spannungsreicher ausfallen kann.

Im Gesamtbild wird deutlich, dass bürokratische, administrative Hürden und disparate Standpunkte bei der Projektrealisierung die häufigsten Probleme darstellen. Es liegt nahe, sie auf im Vorfeld der Kooperationen nicht oder unzureichend erfolgte Klärungen und Absprachen zurückzuführen. Des Weiteren werden im Rahmen einiger Kooperationen ein Mangel an Aufgeschlossenheit und Unvoreingenommenheit und zum Teil asymmetrische Einflussnahmen registriert, die einer partnerschaftlichen Zusammenarbeit auf „Augenhöhe“ entgegenstehen. Die Existenz dieser Barrieren ist bekannt (I. 2.1; BMFSFJ 2017: 211-213); sie verhindern stabile Kooperationen und sollten daher gezielt abgebaut werden. In Anbetracht dessen könnten sich eine aktive Gestaltung und Moderation der Zusammenarbeit zwischen MSO und ,etablierten ' ressourcenstärkeren Einrichtungen, wie etwa Wohlfahrtsoder Jugendverbänden, z. B. durch kommunale Koordinatoren als effektiv erweisen. So könnten etwa über regelmäßig stattfindende Veranstaltungen Kennenlern-, Austausch- und Mitgestaltungsmöglichkeiten erweitert und gemeinsame Projektideen entworfen werden. Diese Plattformen könnten dann auch die Auslotung von geteilten thematischen Interessen, Zielen, gegenseitigen Erwartungen sowie die 
Verdeutlichung von Mehrwerten der Zusammenarbeit ermöglichen und die Ausgangslage und Voraussetzungen für Kooperationen dadurch insgesamt verbessern helfen.

\section{Einschätzung der eigenen Arbeit und Situation}

- Divergierende und differenzierte Wahrnehmungen durch Funktionsträger

72 Prozent der befragten Funktionsträger stimmten der Aussage zu, dass die Arbeit ihrer Organisation professioneller und qualifizierter sein müsste, und 66 Prozent sind der Auffassung, dass die Tätigkeiten der MSO zu wenig öffentliche Wertschätzung und Anerkennung erfahren. Im Gegensatz zur als mangelhaft eingeschätzten Wertschätzung geben 59 Prozent der Befragten an, mit der Einbindung ihrer Organisation seitens der Stadt zufrieden zu sein, und mehr als die Hälfte (56\%) bestätigt, dass in ihrer Organisation bekannt ist, wo und wie Fördermittel beantragt werden können. Die beiden Aussagen, dass ihre Organisation in einem starken Ausmaß mit Fremdenfeindlichkeit und Diskriminierung konfrontiert und dass die öffentliche Meinung über ihre Gruppe negativ ist, werden mit jeweils über 70 Prozent verneint. Circa ein Fünftel der MSO (21\%) registriert Konkurrenzverhältnisse zu anderen MSO.

- Unterschiedliche Wahrnehmungen: Fremdenfeindlichkeit (RMO) versus Konkurrenz (MO)

Während die Wahrnehmung einer Konkurrenzsituation zu anderen MSO bei den säkularen MSO signifikant stärker ausfällt, ist bei den religiösen MSO hingegen die Wahrnehmung von Fremdenfeindlichkeit bzw. Diskriminierung sowie einer negativen öffentlichen Meinung signifikant stärker ausgeprägt. Diese Ergebnisse sind nicht überraschend, da Muslime und in erster Linie islamische MSO, die auch in der Substichprobe der religiösen MSO dominant vertreten sind, mit Vorbehalten, Misstrauen und Negativ-Zuschreibungen konfrontiert sind (vgl. Pollack/Müller 2013: 43; Pollack 2014b: 34; Vopel/El-Menouar 2015; Nagel 2015b: 31; II. 3.2). Die Studie zeigt, dass Labelig- und Zuschreibungsprozesse, die religiös gebundene Individuen betreffen, auch für die Organisationsebene gelten. Diese Zuschreibungen vollziehen sich auf der Wahrnehmungsebene und können gesamtgesellschaftlich schädliche Folgen haben. Anhand der Organisationsbefragung zeigt sich mit Blick auf bestehende Vorurteile, abwertende Einstellungen, Diskriminierungen und Ausgrenzungen von insbesondere als „,fremd“ oder „,anders“ empfundenen (muslimischen) Individuen und Gruppierungen gesellschaftspolitischer Handlungsbedarf. Dieser wird umso 
deutlicher, wenn man - jenseits dieser Befragungsergebnisse - die jüngst ermittelte Stabilisierung fremden-, islam- und demokratiefeindlicher Einstellungen in Deutschland berücksichtigt (vgl. u. a. Zick et al. 2019a; Pollack et al. 2014). Angesichts der Stereotypisierungen, die allerorten üblich sind, könnten sich Maßnahmen als hilfreich erweisen, mittels derer diese Wahrnehmungsroutinen in der Öffentlichkeit zur Sprache gebracht und damit einhergehende Schieflagen reflektiert und hinterfragt werden können. Relevant werden damit die Kommunikation mit und nicht über Religionsgruppen sowie die Ermöglichung sozialer Kontakte über den Aufbau von Gelegenheitsstrukturen für gesellschaftliche Dialoge und Begegnungen, um insbesondere gegenüber Muslimen und Juden bestehende Vorurteile abzubauen.

Auch hinsichtlich des Umgangs mit mangelhafter öffentlicher Wertschätzung und städtischer Einbindung ist von erwünschten Optimierungen und Entwicklungspotenzialen auszugehen. Darüber hinaus verweisen die Ergebnisse auf weitere Unterstützungs- und Verbesserungsbedarfe: Hinsichtlich der Behebung von konstatierten Professionalisierungs- und Qualifizierungsdefiziten könnten sich für die betreffenden MSO jeweils relevante thematische Qualifizierungen und Weiterbildungen in ausgewählten Handlungsfeldern als effektive Maßnahmen erweisen, damit sich die MSO in ihrem Tun und Wirken langfristig kompetenter und sicherer fühlen. Auch könnten durch solche Maßnahmen ihre Handlungsspielräume und sozialen Wirkungsbereiche, ihre Zugangsmöglichkeiten und Partizipationschancen erweitert werden.

- Keine negativen Auswirkungen von Fremdenfeindlichkeit und gesellschaftlichen Fehlplatzierungen auf Kooperationsverhalten

Die zur Prüfung der Auswirkung wahrgenommener Fremdenfeindlichkeit, Diskriminierung und öffentlicher Negativ-Diskurse formulierte Doppelhypothese 5 ( $\mathrm{H} 5_{1}$, $\left.\mathrm{H} 5_{2}\right)^{146}$ zeigte, dass sich die beiden Phänomene nicht negativ auf das unter den MSO grundsätzlich stark ausgeprägte Kooperationsverhalten auswirken. Die Ergebnisse sprechen eher dafür, dass die MSO auch bei Wahrnehmung der beiden negativen Phänomene grundsätzlich mit anderen Einrichtungen zusammenarbeiten und dass die Perzeption von Herabsetzungen und fremdenfeindlichen Gesinnungen demnach insgesamt nicht als für Nicht-Kooperationen ursächlich betrachtet werden kann.

${ }^{146}$ H5 $_{1}$ : Die Wahrnehmung von Fremdenfeindlichkeit und Diskriminierung führt mit großer Wahrscheinlichkeit dazu, dass sich MSO zurückziehen und keine Kooperationen eingehen.

H5 $_{2}$ : Die Wahrnehmung von negativen öffentlichen Zuschreibungen und Diskursen bewirkt mit großer Wahrscheinlichkeit, dass MSO verstärkt Kooperationen eingehen. 
Mit großer Sicherheit ist davon auszugehen, dass die beiden betrachteten Phänomene (Fremdenfeindlichkeit; ablehnende Diskurse) nicht von den jeweils gewählten Kooperationspartnern ausgehen.

Anhand der Testergebnisse kann jedoch nicht auf kausale Zusammenhänge im Sinne von Ursache und Wirkung geschlossen werden: Es kann nicht festgestellt werden, inwieweit das Kooperationsverhalten eine bewusste, strategische Reaktion auf registrierte gesellschaftliche Fehlplatzierungen darstellt und inwieweit die Phänomene tatsächlich aufeinander bezogen sind.

- Andere MSO und Kirchen als potenziell Verbündete bei Wahrnehmung von Fremdenfeindlichkeit und gesellschaftlichen Fehlplatzierungen

Bei existentem Kooperationsverhalten wurde auch der Typus des gewählten Kooperationspartners untersucht. Auch hier ließen sich keine negativen Zusammenhänge erkennen. Hinsichtlich der beiden Kooperationstypen ,andere MSO“ und „Kirche“ kann aufgrund statistisch signifikanter Prüfergebnisse (7.1.7) jedoch angenommen werden, dass mit diesen scheinbar gerade bei Konfrontation mit xenophoben Haltungen und unzutreffenden öffentlichen Meinungen zusammengearbeitet wird, d. h. dass sich die betroffenen MSO die Einrichtungen ggf. gezielt als Verbündete suchen.

Die Tatsache, dass bei Negativ-Zuschreibungen kooperiert wird, negiert die Annahme von allgemeinen Rückzugstendenzen aufgrund wahrgenommener Abwertungen der eigenen Gruppe. Die beiden Phänomene Diskriminierung/Fremdenfeindlichkeit und negative Meinungen resultieren nicht - wie mit der Doppelhypothese konstruiert - in unterschiedlichem Kooperationsverhalten, sondern besitzen den gleichen Stellenwert.

- Ausgeprägtes Kooperationsverhalten von miteinander konkurrierenden MSO

Des Weiteren konnte auch die Hypothese 7, die von erhöhter Kooperation mit städtischen und staatlichen Behörden seitens miteinander in Konkurrenz stehender MSO ausging, nicht bestätigt werden. Denn es ließen sich keine signifikanten Ergebnisse hinsichtlich der Wahrnehmung einer Konkurrenzsituation unter den MSO und der Zusammenarbeit mit städtischen oder staatlichen Organisationen identifizieren. Hingegen scheinen Wohlfahrtsverbände bei wahrgenommenen Konkurrenzsituationen mit anderen MSO wichtige Kooperationspartner darzustellen. Paradoxerweise ist unter den MSO, die mit anderen migrantischen Vereinigungen zusammenarbeiten, das Merkmal des In-Konkurrenz-Stehens signifikant höher ausgeprägt als unter den mit anderen MSO nicht-kooperierenden.

Das Phänomen der erhöhten und bevorzugten Kooperation mit Wohlfahrtsverbänden - bei einer wahrgenommenen Konkurrenzsituation mit anderen MSO - ist 
auch im Zusammenhang mit der Beantwortung der Frage nach der Mitgliedschaft in Dachverbänden zu erklären. Hier wurde am häufigsten die Mitgliedschaft im Paritätischen Wohlfahrtsverband angeführt. In diesem Zusammenhang ist bedeutsam, dass den Spitzenverbänden der Freien Wohlfahrtspflege und insbesondere dem Paritätischen Wohlfahrtsverband bei der Integrationsarbeit sowie Beratung und Förderung von MSO im Bundesland NRW eine zentrale Funktion zukommt (vgl. Halm et al. 2020: 122).

Hinsichtlich des Widerspruchs, dass untereinander in Konkurrenz stehende MSO miteinander kooperieren, wurde die Möglichkeit der Existenz eines Kooperationswettbewerbs (engl. ,,co-opetition“) ins Spiel gebracht (Jansen et al. 2000). Denn es erscheint lukrativ, dass sich Konkurrenten in unabwendbaren Wettbewerbssituationen verbünden, um Interessen zu bündeln, Vorteile und Erfolge zu erzielen und Außenseiterpositionen, die aus Diskriminierungserfahrungen resultieren können (s. o.), zu vermeiden. Eine solche Konstellation kann sich dann - auch für die betreffenden MSO in NRW - als vorteilhafte Konstellation, als eine Win-win-Situation, erweisen.

\section{- Fazit}

Eigenständige Profile religiöser und säkularer MSO: Spezifische Binnen- und Außenverhältnisse

In dieser Arbeit ging es konsequent darum, die zivilgesellschaftlichen Performanzen aller MSO im Gesamtbild sowie Unterschiede zwischen religiösen und säkularen Vereinigungen in den Blick zu nehmen. In diesem Kontext lautet die anhaltend virulente Frage: Inwieweit lässt sich der „Faktor Religion“ für zivilgesellschaftliche Performanzen eher als eine förderliche oder hemmende Größe einstufen und welche Spezifika und Diskrepanzen sind im Vergleich von religiösen mit nicht-religiösen Vereinigungen erkennbar?

Die berichteten Ergebnisse unterstreichen spezifische Eigenschaften und Präferenzen der beiden Organisationstypen als Kollektivakteure und tragen zu ihrer Profilierung bei. Ersichtlich ist insbesondere ein charakteristisches Profil der religiösen Vereinigungen: Dieses markiert eine eigenständige Qualität und Bedeutung von Religion für die Gemeinschaft(sbildung) und eine kollektive soziale Identität, die das gemeinsame Handeln motivieren und konturieren.

Bei den befragten religiösen MSO sind eine höhere personelle sowie finanzielle Ressourcenausstattung sowie weitere Besonderheiten erkennbar: Die religiösen MSO verfügen über mehr Mitglieder, mehr Engagierte, häufiger über Räumlichkeiten sowie über deutlich höhere finanzielle Mittel, die v. a. aufgrund von 
Mitgliedsbeiträgen sowie Spenden und Sponsorengeldern verfügbar sind; sie gewinnen mehr Ehrenamtliche und sind hinsichtlich der Anzahl an Tätigkeitsbereichen aktiver als nicht-religiöse MSO. In der Flüchtlingshilfe verfolgen sie gemeindenahe Unterstützungsleistungen. Religiöse Aktivitäten, Wertüberzeugungen und konfessionelle Homogenität der Mitglieder spielen bei ihnen erwartungsgemäß eine größere Rolle. Im Großen und Ganzen gehen die religiösen Gemeinschaftsbindungen nicht ausschließlich mit Binnenorientierungen, sondern mit einem auch auf die Aufnahmegesellschaft gerichteten Aktivitätsspektrum einher.

In Hinblick auf das Eingehen von Kooperationen und damit bewusst verfolgter Ziele sind die religiösen wesentlich selbstgenügsamer und weniger ambitioniert als die taktisch agierenden säkularen MSO; die religiösen Vereinigungen kooperieren bevorzugt mit Kirchen. Im Gegensatz zu den säkularen MSO erfahren sie im Rahmen der Zusammenarbeit dadurch weniger Konkurrenz und Abhängigkeit.

Ihr religiöser Charakter bedingt, dass sie weniger öffentliche Fördermittel erhalten; im Rahmen der Flüchtlingshilfe haben sie seit 2015 bis zum Zeitpunkt der Befragung (2018) weniger stadtpolitische Aufmerksamkeit und Wertschätzung sowie eine geringere Intensivierung der Zusammenarbeit mit städtischen Ämtern und Behörden erfahren. Auch nehmen sie im Vergleich zu den säkularen MSO mehr Fremdenfeindlichkeit und Diskriminierung sowie im stärkeren Ausmaß eine negative öffentliche Meinung über die eigene Gruppe wahr. Bei der Mobilisierung von Engagierten zeigt sich die Relevanz der religiösen MSO als Gemeinschaften, die die ganze Familie einbinden.

Die nicht-religiösen MSO zeigen mit der Verfügbarkeit von signifikant mehr Hauptamtlichen und einem diesbezüglich positiven Entwicklungstrend professionelle Strukturen (vgl. auch Halm et al. 2020: 119; Friedrichs et al. 2020: 45). Diese bedingen ihre effizienzorientierten und strategischen Ausrichtungen, die sich zum einen in den stärker an struktureller Integration orientierten Aktivitäten spiegeln. So sind für die säkularen MSO die Tätigkeitsbereiche der Bildung bzw. Erziehung sowie des Arbeitsmarktzugangs signifikant bedeutsamer und in der Flüchtlingshilfe zeigen sie eine höhere Affinität zu rechtlich-administrativen Unterstützungsleistungen. Zum anderen gehen die säkularen MSO mit vielen divers fachkundigen Einrichtungen Kooperationen ein und verfolgen mit dieser Zusammenarbeit strategische Ziele. Auch anhand der mitgeteilten Selbstverständnisse ist für die säkularen Vereinigungen ein hohes Interesse an Austausch und Vernetzung mit anderen Akteuren zu konstatieren; gleiches gilt für das Anliegen, Prozesse aktiv zu unterstützen bzw. als misslich wahrgenommene Gegebenheiten zu verbessern. Das Bedürfnis, vorgefundene Zustände verändern zu wollen, zeigt sich auch in den Motiven, die für in säkularen MSO engagierte Personen seitens der Funktionsträger geltend gemacht werden. Zugleich wird hinsichtlich einer erfolgreichen Rekrutierung 
von Engagierten die Relevanz des Angebots von Gegenleistungen und damit eine Profitorientierung deutlich.

Durch ihre stärkere politische Anerkennung und Involvierung ergeben sich für die säkularen Vereinigungen im Rahmen ihrer Kooperationsarbeit mehr Konkurrenzsituationen und Abhängigkeitsverhältnisse und ein stärkerer Wettbewerb mit anderen MSO.

\section{Religion und Konvivialität als Komplementärerscheinungen mit immanentem Kollektivbezug}

Die Auswertungsergebnisse haben verdeutlicht, dass sich religiöse MSO hinsichtlich der Organisationsziele, der Aktivitäten wie auch der Motivstrukturen auch als gesellige und soziale Orte gerieren. Dies ist insbesondere an dem für sie signifikant bedeutsameren Ziel, einen Ort der Geselligkeit und der Zusammenkunft zu schaffen ersichtlich, aber auch daran, dass spezifische Aktivitäten wie Freizeit und Geselligkeit oder Freizeitangebote im Rahmen der Flüchtlingshilfe für sie nicht weniger bedeutsam sind; zudem wird persönliche Vergnügung (,Freude haben“) für Engagierte in religiösen MSO als nicht weniger wichtig erachtet. ${ }^{147}$ Theoretisch wäre anzunehmen, dass Aktivitäten der Freizeit, Vergnügung und Geselligkeit in religiösen Gemeinschaften einen untergeordneten Stellenwert besitzen, da sie mit religiösen (orthodoxen) Wertorientierungen als normative, handlungsleitende Grundprinzipien weniger gut zu vereinbaren sind (II. 4.3.3.2). Eine solche negative Abgrenzung lässt sich in den Daten allerdings nicht erkennen.

An diesem Punkt ist herauszustellen, dass die Relevanz von geselligem Beisammensein und Unterhaltung in religiösen Organisationen in den auf einem (zu) hohen Abstraktionsniveau verhafteten Organisationstheorien bzw. innerhalb typenspezifischer Organisationskonzeptionen entweder gar nicht oder nur am Rande thematisiert wird. ${ }^{148}$ Diese fokussieren auf die Verinnerlichung von handlungsregulierenden religiösen Normen, die Konformität mit moralischen Vorschriften und Verpflichtungen und die Relevanz von sozialer Kontrolle für die Einhaltung religiöser Normen sowie die Gewährleistung des Gruppenzusammenhalts. In religionssoziologischer wie lebenspraktischer Hinsicht ist der Befund gleichwohl eingängig. Wie in der

\footnotetext{
${ }^{147}$ Freizeit \& Geselligkeit (RMO: $82 \%$ vs. MO: $77 \%$ ); Gemeinsame Freizeitgestaltung (78 \% vs. $70 \%$ ); Freude an der Tätigkeit haben (96\% vs. $93 \%$ ).

${ }^{148}$ Vgl. dazu Clark/Wilson 1961; Barnard 1970; Simon 1981/[1945]; March/Simon 1976/[1958]; Parsons 1960; Etzioni 1975; Blau/Scott 1962; Beckford 1975; Demerath et al. 1998; Sanders/Kianty 2006; Scott/Davis 2007; Beckford/Richardson 2007; Petzke/Tyrell 2012; Kühl 2015; Wilson 2020.
} 
Einleitung (I. 1) und in anderen Kapiteln dieser Arbeit expliziert, lässt sich ein transzendentes Bezugssystem in der Immanenz, d. h. in der weltlichen Lebenspraxis kommunikativ erfahrbar und präsent machen, worauf es bei der in Rede stehenden Performanz der religiösen MSO ankommt. Insbesondere die gemeinschaftliche Vergegenwärtigung und das kollektive Erleben des Religiös-Transzendenten können im Alltag Energien und Emotionen freisetzen, die Frohsinn und Behagen in und an der Gemeinschaft evozieren.

Die von Religionen gesetzten, als verbindlich akzeptierten moralischen Prämissen bzw. ethisch-sittlichen Normen und Grundsätze regulieren das Verhalten der einzelnen Gemeinschaftsmitglieder und beziehen sich auf das Kollektiv. Glaube und Religionspraxis sind unmittelbar in die kollektiven Erfahrungen und Emotionen, die die soziale Gruppe ermöglicht und gewährleistet, eingebettet und auch im weltlichen Diesseits verankert. Religion ist somit ein kollektiv erlebbares Phänomen, das Solidarität und Zusammenhalt ermöglicht (Durkheim 1984/[1912]: 580 ff.; Helle 1997: 28). Religionspraxis und Sozialität sind folglich aufeinander bezogen und insbesondere in der Migrations- und Minderheitensituation mit Bedürfnissen nach Zusammenkunft und Konvivialität im institutionalisierten Rahmen verknüpft.

Neben dieser genuin religiösen Verortung wurde jedoch ebenfalls dargelegt, dass persönliche Vergnügung auch mit alltags,,weltlichen“ Anliegen, die z. B. eine gemeinsame Freizeitgestaltung betreffen, zu erklären ist. Dies bedeutet, dass theoretische Mutmaßungen über eine Unverträglichkeit von je spezifisch gearteten religiös und nicht-religiös konnotierten Beweggründen an dieser Stelle nicht berechtigt ist.

\section{Vitale zivilgesellschaftliche Performanz statt Bonding-Affinität der religiösen MSO}

Im Rahmen der Auswertung von Bridging- und Bonding-Performanzen ließ sich keine besondere Bonding-Affinität der religiösen MSO feststellen. ${ }^{149}$ Auch in Hinblick auf die herausgearbeiteten Bonding- und Bridging-Typen sind für die in der Analysestichprobe zahlenmäßig stärker vertretenen einzelnen Religionstraditionen (Islam, Alevitentum, Christentum) keine spezifischen Tendenzen auszumachen. Anhand multipler Regressionsanalysen zeigte sich jedoch, dass Bonding-Ziele für die nicht-islamischen Religionen gewichtiger sind. Dies impliziert eine größere Bedeutsamkeit eines ,eigenkulturellen Rückzugsort[s]“ (Baumann 2004: 27) und

\footnotetext{
${ }^{149}$ Die befragten religiösen MSO tendieren im höheren Ausmaß als die säkularen Organisationen dazu, als eine Mitgliedschaftsvoraussetzung ,die Zugehörigkeit zu einer bestimmten Glaubensgemeinschaft" und als ausschließliche Zielgruppen ihrer Aktivitäten und Angebote „Personen mit den gleichen religiösen Überzeugungen“ anzugeben (7.2.3.2). Dieses resultiert im Gesamtbild jedoch nicht in einer abgrenzenden Performanz (7.2.4).
} 
der Stärkung der eigenen Gemeinschaft im Binnenraum der nicht-islamischen MSO. Dadurch wird die öffentliche Debatte um, den problematischen Islam ' konterkariert.

Darüber hinaus wurde deutlich, dass den religiösen Eigenschaften eigenständige Effekte zukommen, die mit anderen Bonding- und v. a. auch Bridging-Ausprägungen kompatibel sind bzw. letztere nicht abschwächen. Die empirischen Befunde dieser Studie, die über vergleichende, kleinschrittige Analysen ermittelt wurden, sind insofern als Anhaltspunkt für ähnliche zivilgesellschaftliche Performanzen von religiösen und säkularen MSO zu betrachten, sobald religionsbezogene Variablen in der Auswertung unberücksichtigt bleiben.

Die genauere Betrachtung der jeweiligen Eigenschaften der nach bonding und bridging typisierten MSO (7.2.5) hat nahegelegt, dass es die in Kombination mit einer religiösen Orientierung zutage tretenden personellen Ressourcen (Hauptamtliche, Engagierte) sowie eine teilweise auch von Engpässen flankierte finanzielle Stärke zu sein scheinen, die die religiösen MSO befähigen, gleichermaßen sowohl nach innen bindend als auch nach außen beziehungsstark und vielfältig aktiv zu sein. Dabei ist die Verfügbarkeit personeller Ressourcen bedeutsamer als das Vorhandensein von als ausreichend empfundenen finanziellen Mitteln. Die kollektive religiöse Identität und die Einbindung der Mitglieder bzw. Engagierten in die religiöse Gemeinschaft scheinen vitalen zivilgesellschaftlichen Performanzen förderlich zu sein (vgl. Liedhegener et al. 2019: 27 ff.). Hier ist mit Antonius Liedhegener et al. (ebd.: 45) zu schlussfolgern, dass Religion ,,auf der individuellen Ebene der Religiosität über die Religionszugehörigkeit dem ehrenamtlichen Engagement förderlich“ ist und sie ,auf der strukturellen Ebene der Organisation von zivilgesellschaftlichem Engagement" wirkt (vgl. II. 4.2.4, auch Halm et al. 2012a: 421 f.; Halm/Sauer 2015; Klie 2018: 441 ff.; Nagel 2015a). Insofern kann anhand der empirischen Befunde der vorliegenden Arbeit die Bedeutung von religiösem Sozialkapital als ein wichtiger Faktor für gesellschaftliche Integrationsprozesse verdeutlicht werden.

In diesem Zusammenhang zu erwähnen sind ebenfalls die Ergebnisse der im Rahmen der Untersuchung von Bonding- und Bridging-Performanzen durchgeführten multiplen Regressionsanalysen. Sie sind in methodischer Hinsicht nicht vergleichbar mit den im Kontext der Typenbildungen zutage getretenen, soeben dargelegten Resultate. Anhand der Regressionen konnte zum einen ermittelt werden, dass Sozialkapital-Profile und Handlungsfähigkeit der MSO nicht durch personelle Ressourcenstärke, d. h. die Verfügbarkeit von Engagierten und Hauptamtlichen, (prä-)determiniert oder eindeutig vorhersagbar sind (7.2.5.4). Es lässt sich nicht feststellen, dass es ohne Hauptamtliche kein Bridging gibt oder - umgekehrt - dass, wenn Bridging-Ausprägungen vorhanden sind, dann auch Hauptamtliche verfügbar sind. Insofern kann angenommen werden, dass auch bei personell nachteiligeren 
Verhältnissen und geringeren Handlungsspielräumen, ggf. auf Basis gemeinsamer Wertegrundlagen, zivilgesellschaftliche Vitalität entstehen kann, wenn dafür seitens der MSO bzw. der in ihnen involvierten Personen Bedarfe und Realisierungsmöglichkeiten gesehen werden. Eine solche Interpretation zeigte sich auch bei Betrachtung der organisationalen Eigenschaften der Bonding- und Bridging-Typen als plausibel. Zum anderen zeigte sich im Kontext der Prüfung der Hypothese 2, mit der - unabhängig von der Bonding/Bridging-Analyse - angenommen wurde, dass ressourcenstärkere MSO eine höhere Anzahl von Aktivitäten und ein größeres Spektrum an Kooperationspartnern aufweisen ${ }^{150}$, jedoch auch, dass die personelle Ausstattung mit Hauptamtlichen die Anzahl der Kooperationspartner der MSO erhöht und die Hauptamtlichen folglich durchaus als eine bedeutsame Einflussgröße einzustufen sind (7.1.6).

Das auf religiöse Eigenschaften zurückzuführende, Dynamiken und Energien freisetzende Performanz-Profil der religiösen MSO tritt zusammen mit einer habituellen Selbstgenügsamkeit, Zurückgezogenheit bzw. Selbstreferentialität in Erscheinung, wie dies v. a. anhand des Kooperationsverhaltens sichtbar wurde. Daran zeigt sich die charakteristische „Doppelgesichtigkeit“ religiöser Vereinigungen, wobei dieser Spannungszustand bei den im Rahmen dieser Arbeit befragten MSO mit an der Aufnahmegesellschaft orientierten - brückenbildenden - Tätigkeiten einhergeht. Insofern sind Anknüpfungspunkte an die Mehrheitsgesellschaft und eine Deutschlandorientierung gegeben. Sollte dies als eine allgemein geltende Tendenzaussage einzustufen sein, ist gleichwohl zu konstatieren, dass es in Deutschland zweifelsohne migrantische religiöse Gemeinschaften gibt, in denen autoritäre, fundamentalistische, demokratiedistante Einstellungen und problematische Geschlechterrollen propagiert und gepflegt werden. Diese stehen insbesondere in Zusammenhang mit strikter, traditionalistischer muslimischer Religiosität und zeigen sich in Deutschland und international anhand dynamischer radikaler islamistischer Strömungen wie dem Salafismus (Toprak/Weitzel 2019; Ceylan/Kiefer 2017a, 2013; Kiefer et al. 2017). Solche Phänomene müssen benannt und dürfen nicht ausgeblendet werden, da sie sich kohäsionsgefährdend und segregationsfördernd auswirken. Jedoch greift es zu kurz, diesbezügliche Ursachen allein in einer stark ausgeprägten antimodernistischen Religiosität zu suchen; vielmehr sind sozioökonomische Bedingungen der Lebensumwelt, Orientierungs- und Wertekrisen, Ausgrenzungs- und Benachteiligungserfahrungen, geringe soziale Aufstiegschancen und weitere gesellschaftliche Rahmenbedingungen in Hinblick auf komplexe

${ }^{150}$ H2: Insbesondere die mit genügend Geld und personellen Ressourcen (Mitglieder, Hauptamtliche und Engagierte) ausgestatteten MSO sind imstande, ein breites Spektrum an Aktivitäten und Dienstleistungen anzubieten und Kooperationen einzugehen. 
individuelle und kollektive Identitätsbildungsprozesse im Kontext kultureller Differenz mitzuberücksichtigen (u. a. Heitmeyer et al. 1997; Uslucan 2016; Pollack et al. 2016; s. auch II. 4.2.5).

Die Existenz dieser offensichtlich problematischen, konfliktiven Erscheinungen ist herauszustreichen, insbesondere da sie sich auch in muslimischen Organisationen finden lassen, sie mit dieser Arbeit aber nicht abgebildet werden konnten (5.3; 7.2.6); insofern ist es nicht das Anliegen der Autorin, dass die Befunde ihrer Studie in Hinblick auf Integrationsgeschehen als allgemeingültige positive Beurteilung der Ausrichtungen und Performanzen migrantischer Organisationen rezipiert werden.

\section{Gemeinsamkeiten religiöser und nicht-religiöser MSO}

Zweifelsohne kann Religion als Fundament und Element organisationaler Performanz betrachtet werden, die sinnfälliger Weise in Sonderheit für Szenarien und Rahmenbedingungen in Organisationen mit einem expliziten religiösen Traditionsbezug relevant und gängig ist. Dies ist - wie expliziert wurde - auf das intrinsisch verankerte Anliegen zurückzuführen, sich in der Gemeinschaft mit dem Transzendenten, Unverfügbaren ins Verhältnis zu setzen und letzte (Sinn-)Fragen menschlicher Existenz zum Ausdruck kommen zu lassen. Religiöse Komponenten sind aber auch ,,weltlichen“ Unternehmungen nicht fremd und können dort individuelle und soziale Dynamiken auslösen. Formen religiöser Kommunikation und Praktiken sind deshalb nicht auf religiöse Vereinigungen zu beschränken, sondern können auch in säkularen eine Rolle spielen. Die religiösen Haltungen und Handlungen lassen sich als soziale Routinen und Praktiken, d. h. als subjektiv und kollektiv in Anspruch genommene Sinnzusammenhänge verstehen, die auf unterschiedlichen Ebenen und in variierenden Intensitätsgraden von Belang sind. Zum anderen ist - umgekehrt - auch im religiösen Bereich davon auszugehen, dass sich Handlungslogiken vermischen, in dem Sinne, dass religiöse Performanzen nicht die einzigen sind und auch eine abgeschwächte Rolle spielen können. Eine Reduzierung ausschließlich auf religiöse Merkmale wird ihrer Charakterisierung nicht gerecht, da sie in nicht geringem Umfang auch andere, nicht genuin religiös konnotierte Interessen verfolgen. Insofern muss die Bedeutung der (Nicht-)Zuordnung zu einer religiösen Tradition in Bezug auf theoretisch jeweils zu erwartende (nicht-)religiöse Performanzen und konsequente Wenn-Dann-Verkettungen relativiert werden und sollte nicht zur Annahme verleiten, dass sich religiöse Organisationen konsequent religiös gerieren und dass Religion in säkularen Vereinigungen nicht von Belang ist, da in diesen auf kollektiver Ebene durchaus eine Religionsaffinität zutage treten kann. Dies zeigt sich etwa in der Durchführung des interreligiösen Dialogs (30 \%), in einer konfessionell geprägten, homogenen Mitgliederstruktur (20\%), in der Bedeutung 
religiöser Brauchtumspflege (17\%) sowie religiöser Bildung/Erziehung (14 \%) und dem durch den Gottesglauben gefestigten Miteinander (14\%) in MSO, die sich offiziell keiner religiösen Tradition zuordnen.

Ähnlichkeiten und Übereinstimmungen zwischen den separat betrachteten religiösen und profanen Organisationstypen zeigen sich zum einen anhand der nicht signifikanten Ergebnisse statistischer Tests (Unterschiede, Zusammenhänge), z. B. in der Kompatibilität von religiösen und nicht-religiösen Motivstrukturen. Zum anderen wurde empirisch herausgearbeitet, dass die Bedeutung von eigenkulturellem und religiösem Sozialkapital als wichtige Faktoren für gesellschaftliche Integrationsprozesse und insbesondere die religiösem (Sozial-)Kapital innewohnenden brückenbauenden, auch, weltlichen " Potenziale keinesfalls zu unterschätzen sind. Insbesondere auch die Ergebnisse der Analyse von Bondingund Bridging-Sozialkapital legen nahe, dass der Einfluss religiöser Dogmen auf das Handeln von sich einer religiösen Tradition zuordnenden MSO nicht monokausal oder unilinear gedacht werden sollte und sich nicht theoretisch modellieren lässt. Insofern sind die Effekte religiösen Sozialkapitals - bei grundsätzlich möglichen ambivalenten Wirkungskräften - insgesamt differenziert(er) und flexibler zu denken. In diesem Zusammenhang wurde thematisiert (II. 4.3.5), dass Organisationen in diversen gesellschaftlichen Teilbereichen agieren, unterschiedliche Umwelterwartungen, Funktionslogiken, Regeln und Normen zu berücksichtigen und Anpassungsleistungen zu vollziehen haben. Martin Petzke und Hartmann Tyrell sprechen in diesem Zusammenhang vom „Phänomen der organisationellen Multireferentialität“, von „organisationsuntypische[n] Ambivalenzen“ und einer daraus potenziell resultierenden „Selbstsäkularisierung“ (Petzke/Tyrell 2012: 293; s. auch Pickel 2017: 391 f.; Luhmann 1964: 305 f.), die letztlich zu einer Ähnlichwerdung von religiösen und nicht-religiösen Vereinigungen führen können.

Vor dem Hintergrund der empirisch fundierten Erkenntnisse lassen sich dichotomisierende, v. a. theoretisch essentialisierende Betrachtungsweisen nicht halten. Religiöse Vereinigungen lassen sich organisationssoziologisch nicht konsequent als exakt abgrenzbare Sozialgebilde sui generis einordnen. Ähnlichkeiten wie Gemeinsamkeiten von religiösen und nicht-religiösen Organisationen zeigen an, dass die arbeitshypothetisch konstruierten Typen Schnittmengen haben. Durch Gegenüberstellung und Vergleich können abgesonderte Charakteristika abgeschwächt werden. Die Aufdeckung von Gemeinsamkeiten rechtfertigt allerdings nicht, die eigenständigen Profilbilder zu nivellieren; jedoch kann die Annahme, dass zwischen den beiden Organisationtypen erhebliche Differenzen bestehen, relativiert werden. Insofern wird die Grenze zwischen religiösen 
und nicht-religiösen Organisationen durchlässiger, ohne sie auszulöschen (Demerath/Schmitt 1998: 391; vgl. auch Beckford 1975: 22). Dabei ist davon auszugehen, dass die registrierten Ähnlichkeiten und Berührungspunkte auch für nicht-migrantische Vereinigungen geltend gemacht werden können.

\section{- Ausblicke}

Die festgestellten Unterschiede und Gemeinsamkeiten sind - wie in der Diskussion der Ergebnisse gezeigt werden konnte - plausibel. Wenngleich die Tragweite der Ergebnisse vorliegender Arbeit im Sinne von verallgemeinerbaren Erkenntnissen nicht zu überschätzen ist, lassen sich die Einsichten als empirisch fundierte und idealtypische Eigenschaften der MSO für die Weiterentwicklung von Theorie und Forschung, in Wissenschaft und Praxis fruchtbar machen. Als Vergleichs- und Projektionsfolie können die Ergebnisse weiteren vertiefenden Diskussionen und Vergleichsuntersuchungen dienlich sein.

Die empirische Organisationforschung basiert auf reichhaltigen, abstrakten Theorien und verschiedenen Analysetechniken, die mit dieser Arbeit keinesfalls ausgeschöpft wurden. Angesichts des als unzureichend und lückenhaft zu bezeichnenden Theoriestands wäre die theoretisch verankerte Organisationsforschung grundsätzlich gut beraten, den freiwilligen nicht gewinnorientierten Vereinigungen religiöser und säkularer, migrantischer und nicht-migrantischer Provenienz zukünftig mehr Aufmerksamkeit zu schenken. Auch angesichts der empirischen Befunde sind Weiterentwicklungen und Modifizierungen von Theoriebildungen anzudenken.

In dieser Arbeit wurde verdeutlicht, dass für Migrantencommunities im Migrations- und Aufnahmekontext mit ihrer Selbstorganisierung diverse Bedürfnisbefriedigungen erfolgen können: Zum einen können kulturelle Traditionen und Identitäten bewahrt, zum anderen kann veränderten Lebensumständen und Motivationslagen Rechnung getragen werden; durch Interessenartikulation kann die Umwelt beeinflusst und damit potenziell gesellschaftliche Legitimation und Verankerung erlangt werden. In Hinblick auf jeweilige Zielsetzungen, Aktionsformen, ethnische und gemeinschaftliche Werte, Weltbilder, Glaubensinterpretationen und konfessionsspezifische Eigenheiten ist von einer unüberschaubaren Vielfalt auszugehen. Organisations- und religionssoziologische Analyse sollte für diese Vielfalt sensibel und offen sein, unterschiedliche kulturelle und soziale wie auch nicht-normative Bedeutungsdimensionen von Religion berïcksichtigen (vgl. auch Beckford 1975: 19, 21).

Insbesondere in Hinblick auf die Organisationsbildungen von kleinen Religionsgemeinschaften wird zukünftig anhaltend hoher Forschungsbedarf bestehen (vgl. 
Nagel 2015a, 2013a; Hero et al. 2008; Baumann/Stolz 2007; Lehmann 2006). Angesichts der grundlegenden gesellschaftlichen und religiösen Wandlungsprozesse sollten organisationstheoretische und religionssoziologische Analyseperspektiven stärker miteinander verschränkt werden und dadurch gegenseitig voneinander profitieren. Dabei bleibt es angesichts dynamischer Migrationsprozesse, zunehmender gesellschaftlicher Diversifikation und Wertepluralisierung nicht aus, dass interdisziplinäre - v. a. auch migrationssoziologische Blickwinkel - ebenfalls zu berücksichtigen sind.

$\mathrm{Zu}$ thematisieren ist an dieser Stelle die ,eigentümliche Organisationsblindheit“ bzw. „die Unsichtbarkeit der religiösen Organisationen“ (Petzke/Tyrell 2012: 286) insbesondere in der deutschen Religionssoziologie. ${ }^{151}$ Martin Petzke und Hartmann Tyrell zufolge ist sie mit deren Fokussierung auf die Untersuchung des Spannungsverhältnisses von Religion und Moderne und die Mikroebene der Individuen als Träger von Säkularisierungs- bzw. Entkirchlichungsprozessen zu erklären (ebd.). Im Fokus organisationstheoretischer Literatur bzw. systemtheoretischer Organisationssoziologie (nach Luhmann) stehen v. a. die ganz grundsätzlichen Fragen zur Organisationsaffinität von Religionen sowie der Organisierbarkeit von religiösem Handeln und Erleben überhaupt (Luhmann 1972). Besondere Aufmerksamkeit erhielten und erhalten das organisationsdisponierte (westliche) Christentum und die beiden christlichen Großkirchen, wodurch ,ein deutlicher , bias‘ zugunsten des (westlichen) Christentums “ (Petzke/Tyrell 2012: 297) und der Kirchen(-soziologie) festzustellen ist. Insofern thematisiert(e) religionssoziologische Forschung bevorzugt das Verhältnis von Gesellschaft und Kirche und die Frage des religiösen und gesellschaftlichen Wandels mit seinen Auswirkungen auf Sozialformen der christlichen Religion und in diesem Zusammenhang auch für die Organisationsebene der Kirchen (Krüggeler et al. 1999; Luhmann 1972, 1977, 2000).

Vor diesem Hintergrund verwundert es nicht, dass sich die (deutsche) Religionssoziologie anderen Phänomenen gewidmet und nicht in erster Linie die Organisation als charakteristische Ausdrucksform des Religiösen untersucht hat. In einer Organisationsgesellschaft, die unter den Bedingungen religiöser und ethnischer Pluralisierung voraussichtlich noch bunter werden wird, bieten sich jedoch insbesondere hinsichtlich der Untersuchung von intraorganisationalen Beziehungen, Interaktionen, Prozessen und Strukturen für eine organisationsbezogene religionssoziologische Forschung mannigfaltige Anknüpfungspunkte.

${ }^{151}$ Der Religionssoziologe James A. Beckford konstatiert für die religionssoziologische Beschäftigung mit religiösen Organisationen im Allgemeinen den Status eines „Cinderella subject“ (Beckford 1975: 18), eines „Aschenputtel-Themas“. 
Im Rahmen der vorliegenden Schlussfolgerung wurde herausgestellt, dass das „Gesellige“, ,das Soziale“ und Konvivialität im Sinne von Vergnügung und Frohsinn bei der Thematisierung von spezifischen Eigenschaften religiöser Organisationen entweder gar nicht oder nur marginal erwähnt wird. Die organisationstheoretische Literatur diskutiert v. a. abstrakte Faktoren, aus denen sich sozialer Zusammenhalt ergibt, nicht aber konkretere Empfindungen wie Frohsinn, Geselligkeit, kameradschaftliches Miteinander etc. Im Blickpunkt steht vielmehr die Fähigkeit religiöser Überzeugungen und Organisationen, das menschliche Denken und Handeln und Aspekte des sozialen Lebens zu regulieren. Dies ist auf die bereits erwähnte grundsätzliche Frage nach der Organisierbarkeit von Religion bzw. die Eigenlogik religiöser Organisationen zurückzuführen, die teilweise von derjenigen säkularer abweicht, wodurch sich hinsichtlich ihrer Charakterisierung anhand von als typisch erachteten Organisationskriterien eine gewisse Eigensinnigkeit ergibt. In der organisationstheoretischen Beschreibung religiöser (insbesondere kirchlicher) Organisationen dominierende Begriffe lauten: Sakralität, Hierarchie, Bürokratie bzw. Bürokratisierung, Autorität, Säkularisierung, religiöse Arbeitsteilung, Koordination und Kontrolle, institutionalisierte Rollen und Verfahren, Professionalisierung, Rekrutierungsprobleme, ethische Vorschriften, göttliche Verordnung, Heilsweg, religiöse Dogmen, Normen, Symbole und Rituale, Lehrfunktionen, Missionsförderung, Einheitlichkeit und Exklusivität von Lehre und liturgischer Praxis (vgl. etwa Beckford 1975; Wilson 2020). Dabei sind spezifische Rollen und Leistungserwartungen innerhalb religiöser Organisationen - im Kontrast zu anderen Organisationen (z. B. Unternehmen oder Verwaltungen) - hinsichtlich der Existenz unterschiedlicher Mitgliedschaftskreise nicht durchgängig relevant und verbindlich (Wilson 2020; vgl. auch II. 4.3.3; II. 4.3.4). Die Bedeutung von Emotionen wird punktuell erwähnt, indem affektive Neutralität (Parsons 1960) für religiösen Organisationen bzw. für die in ihnen involvierten Personen nicht geltend gemacht wird. Was hinter der Affektivität konkret steckt, wird allerdings nicht genauer thematisiert. Aus religionssoziologischer Sicht versteht sich das Religiöse auch ,gern als ,Gefühlssache ““(Petzke/Tyrell 2012: 293) und erhält in seiner Gefühlsfundierung neben religiösen Wertorientierungen und Bedürfnisbefriedigungen mehr Aufmerksamkeit. Hierin zeigt sich exemplarisch das Potenzial, das in der Verknüpfung von religions-, organisations- und migrationssoziologischen Analyseblickwinkeln hinsichtlich der Bearbeitung von blinden Flecken in der einen oder anderen soziologischen Subdisziplin steckt. Diese Verknüpfung ist - wie die vorliegende Arbeit verdeutlicht hat - unabdingbar angesichts der Vermischung unterschiedlicher Bedürfnislagen, die insbesondere im organisationssoziologischen Sondertypus der migrantischen Vereinigungen zusammenfließen und nicht nur religiösen Ursprungs sind. 
Vor dem Hintergrund der theoretischen Erwägungen könnten sich zukünftig Mitgliederbefragungen in einem Mixed-Methods-Design $-\mathrm{d}$. h. mit quantitativen und qualitativen Erhebungen -, die von einem mehrköpfigen interdisziplinären und interkulturellen Forschungsteam durchgeführt werden, als prononciert erkenntniserweiternd erweisen. Hier könnte es sich angesichts des für einen erfolgreichen Feldzugang wichtigen Vertrauensaufbaus im mehrfachen Sinne als gewinnbringend erweisen, die zu untersuchenden Organisationen von Anbeginn in die Forschungskonzeptionen und -durchführungen mit einzubeziehen (s. hierzu auch BMFSFJ 2017: 491).

Grundsätzlich könnte sich die Durchführung mündlich-persönlicher, mehrsprachiger Befragungen als instruktiv erweisen; in solchen wären unter Verwendung von Befragungsmaterialien (wie z. B. Listen, Bilddarstellungen oder Karten für Sortiervorgänge) auch komplexere Ermittlungen möglich, die die tatsächlichen Gegebenheiten in den MSO ggf. besser abbilden. Eine solche Methode würde eine „repräsentative“ Abbildung von sehr unterschiedlichen MSO gewährleisten, die mit postalischen, telefonischen oder Online-Befragungen nicht entsprechend ihrem tatsächlichen Anteil an der Grundgesamtheit erfasst werden können (dies betrifft z. B. auch Befragte mit niedriger Schulbildung) (vgl. 7.2.6). Dass eine solche Vorgehensweise außerordentlich kosten-, personal-, planungs- und zeitintensiv ist, steht außer Frage.

Aufgrund der starken Präsenz von MSO in NRW, aber auch in anderen Bundesländern, die jeweils sehr unterschiedliche strukturelle Rahmenbedingungen und gesellschaftliche Herausforderungen kennen, sind hinsichtlich weiterer Studien zu MSO, andere thematische Akzentsetzungen sinnvoll. Zu nennen wären z. B. die Auswirkungen einer zunehmenden Konfrontation der MSO mit rechtsextremistischen Einstellungen und Gruppierungen v. a. in ostdeutschen Bundesländern und die Ermittlung von effektiven Gegenstrategien. Dies könnte weitere erkenntnisreiche Resultate für das in der Einwanderungsgesellschaft Deutschland herausgeforderte, aktiv zu gestaltende Zusammenleben zutage fördern und die in Deutschland beheimateten Individuen unterschiedlicher Provenienz sowie politisch verantwortliche Akteure mit Wissen ausstatten, das sie umsichtiger agieren lassen sollte. 
Open Access Dieses Kapitel wird unter der Creative Commons Namensnennung 4.0 International Lizenz (http://creativecommons.org/licenses/by/4.0/deed.de) veröffentlicht, welche die Nutzung, Vervielfältigung, Bearbeitung, Verbreitung und Wiedergabe in jeglichem Medium und Format erlaubt, sofern Sie den/die ursprünglichen Autor(en) und die Quelle ordnungsgemäß nennen, einen Link zur Creative Commons Lizenz beifügen und angeben, ob Änderungen vorgenommen wurden.

Die in diesem Kapitel enthaltenen Bilder und sonstiges Drittmaterial unterliegen ebenfalls der genannten Creative Commons Lizenz, sofern sich aus der Abbildungslegende nichts anderes ergibt. Sofern das betreffende Material nicht unter der genannten Creative Commons Lizenz steht und die betreffende Handlung nicht nach gesetzlichen Vorschriften erlaubt ist, ist für die oben aufgeführten Weiterverwendungen des Materials die Einwilligung des jeweiligen Rechteinhabers einzuholen.

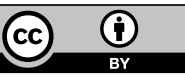

